Portland State University

PDXScholar

Fall 12-12-2012

\title{
Iktomi: A Character Traits Analysis of a Dakota Culture Myth
}

Marianne Sue Kastner

Portland State University

Follow this and additional works at: https://pdxscholar.library.pdx.edu/open_access_etds

Part of the American Literature Commons, and the Other Languages, Societies, and Cultures Commons

Let us know how access to this document benefits you.

\section{Recommended Citation}

Kastner, Marianne Sue, "Iktomi: A Character Traits Analysis of a Dakota Culture Myth" (2012).

Dissertations and Theses. Paper 896.

https://doi.org/10.15760/etd.896

This Thesis is brought to you for free and open access. It has been accepted for inclusion in Dissertations and Theses by an authorized administrator of PDXScholar. Please contact us if we can make this document more accessible: pdxscholar@pdx.edu. 
Iktomi: A Character Traits Analysis of a Dakota Culture Myth

by

Marianne Sue Kastner

A thesis submitted in partial fulfillment of the requirements for the degree of

\author{
Master of Arts \\ in \\ Teaching English to Speakers of Other Languages
}

Thesis Committee:

Keith Walters, Chair

Kimberley Brown

Jennifer Mittelstaedt

Portland State University

2012 
(C)2012 Marianne Sue Kastner 


\begin{abstract}
Cultural folk stories and myths transmit cultural knowledge; however, the manner in which transmission is accomplished has not been satisfactorily resolved. Using three separate English-language versions of a single Dakota story "Iktomi," this study presents a novel qualitative method for analyzing folk tales to discern patterns among renderings of stories by different storytellers in various situations. The author analyzed similarities and differences in the three versions of the tale in order to separate idiosyncrasies related to the different tellers from the broader cultural tale itself.

The study focused on the coding of character traits to elucidate character roles and relationships which reveal how they are used to represent progressive stages in the Dakota vision quest — one of seven sacred rites of the Dakota people. The author systematically analyzed the codes using simple word counts of individual character traits as well as gendered pronouns and enclitics and considered documenter effect on the individually rendered versions. The findings confirm that the story encodes a framework for the vision quest.

The method involved identifying and coding traits exhibited by each story character according to type with regard to representation, ability, or attribute in order to ascertain patterns of relationship among the codes. Then, the reorganization of the coded traits into pairs of polarized correspondences provided more detailed comparison that further clarified relationships among traits. Pronoun and enclitic use pointed to genderspecific activity of characters which analysis of documenter effect reinforced. Emergent patterns from the analyses disclosed a male-specific focus on character activities that
\end{abstract}


delineate an encoded framework for the vision quest regarded tribally as a male rite of passage.

Character traits analysis offers a unique approach for comparing cultural stories rendered in various ways with disparate amounts of detail and for discerning multiple layers of embedded meaning, useful for retrieving, understanding and preserving endangered cultural information. 


\section{Acknowledgements}

Many elements came together to make this project possible and I owe much gratitude to so many for their contribution in helping me develop this work and follow it through to completion. Although I cannot possibly mention all of them here, I acknowledge each one in my mind and my heart with sincerest appreciation and affection.

I express my complete gratitude to the Wakay Tayka- the Great Mystery-for providing me with this amazing opportunity to retrieve a bit of knowledge and wisdom from the ancestors, for making it possible for me to notice and recognize it, and for allowing me to apply it in my own life and share it with others. I thank, in tandem, the many unseen spirit helpers, especially those who helped me "see" and those, seen and unseen, who directed me in arranging the words to describe the vision.

I say thank you to the members of the Native community-elders, teachers, spiritual leaders, friends — who have intentionally or inadvertently provided me with insights and understanding about their culture and way of life and to those who permitted me to participate in their ceremonies. Their acceptance and generosity has made it possible for me to walk this path with Iktomi with a measure of understanding I might not have achieved without their willingness to share their knowledge.

I thank Professor Keith Walters who was a mainstay of positive reinforcement and directed focus throughout the lengthy process, and I also extend heartfelt thanks to my friend and colleague Abigail Pecore who graciously shared her time, exchanging encouragement and support with me as we strove toward mutual goals. 
Finally, I could not have accomplished this work without the grand assistance of that elusive character Iktomi. I am eternally indebted to his artifice and craft in enticing me along the winding path, poking around through piles of data, and dangling such rich and alluring hooks to capture my notice and lead me haphazardly, though assuredly, toward his store of treasure.

To all I say "pilamaya na mitakuye oyas'in." 


\section{Table of Contents}

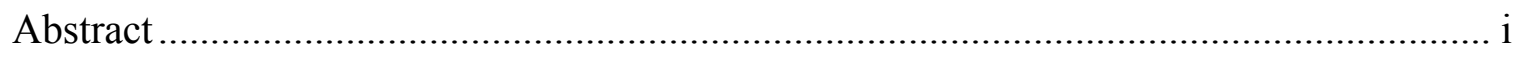

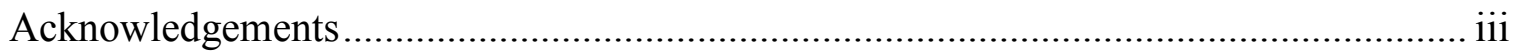

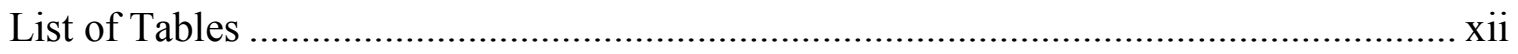

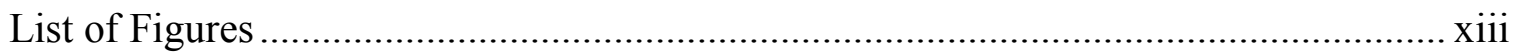

Chapter 1. Iktomi: A Character Traits Analysis of a Dakota Cultural Myth ....................1

Study Rationale, Purpose, and Research Questions ................................................

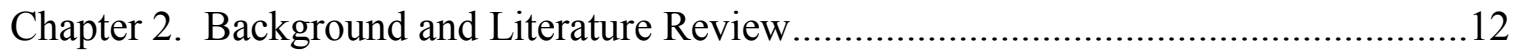

Part 1: Social Theory and Scholarly Approaches to Native American Myths ...............14

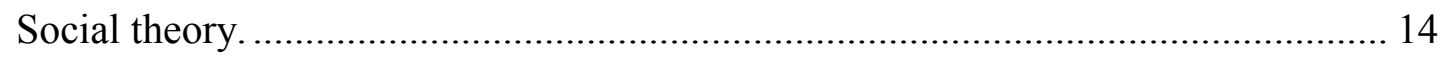

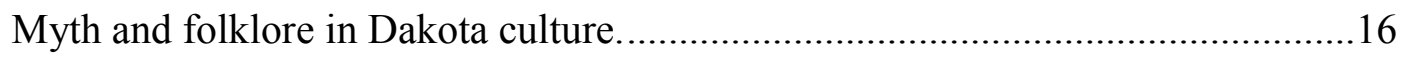

Social role of stories: Perception and value. .............................................16

Myth and socialization of children........................................................ 18

Western perspective: Timeline of Western thought and advent of the Boasian

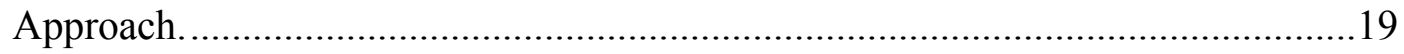

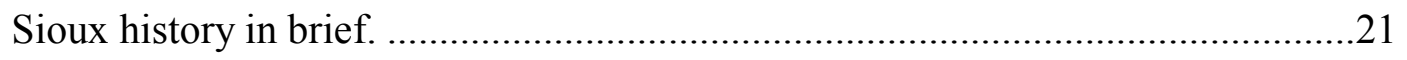

US Indian Policy and Reimaging of Native Americans.................................21

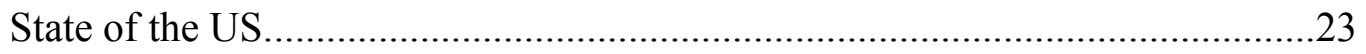

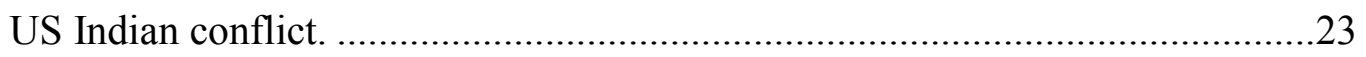

Scholarly approaches to Native American folk myths. ....................................... 27

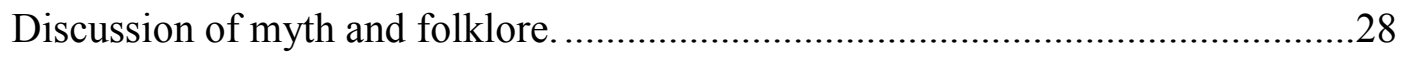

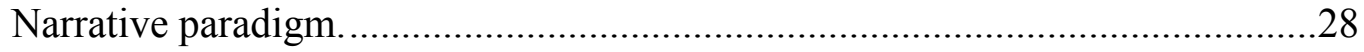




\section{IKTOMI: A CHARACTER TRAITS ANALYSIS}

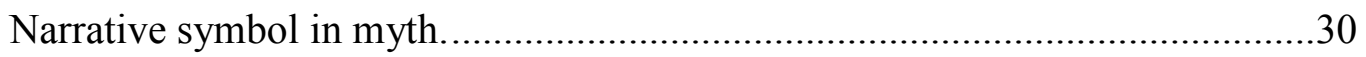

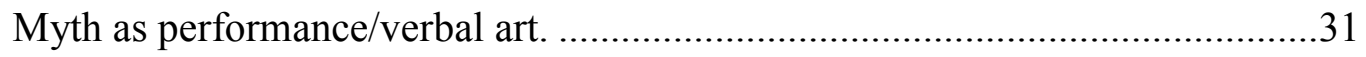

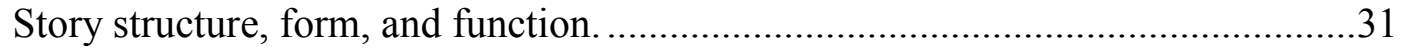

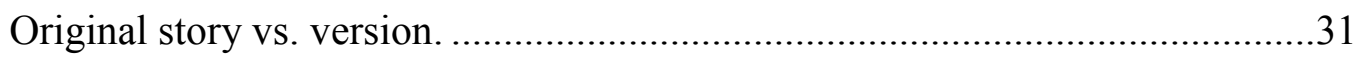

Story Sources: Three versions of the tale.....................................................33

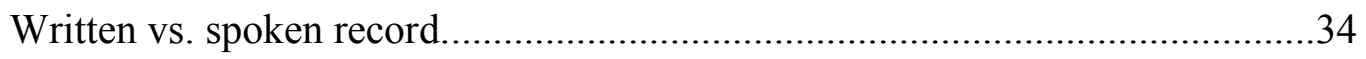

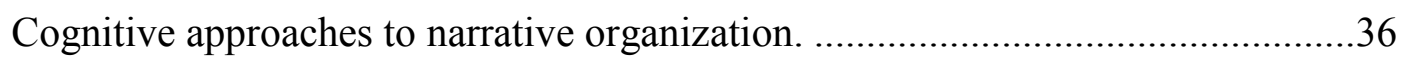

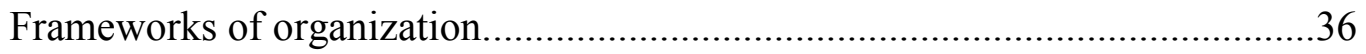

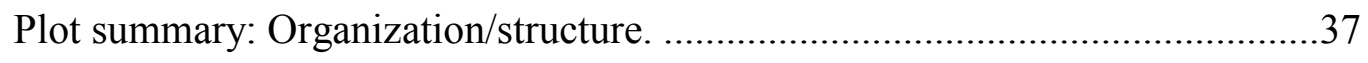

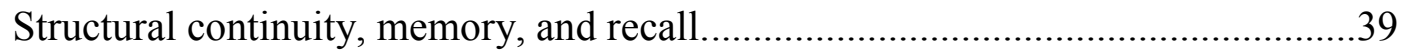

Stylistic form: Performance art and the poetic style debate.............................39

Story comparison across cultures. ....................................................45

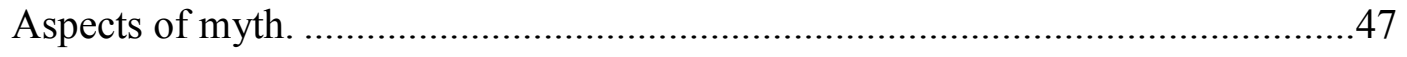

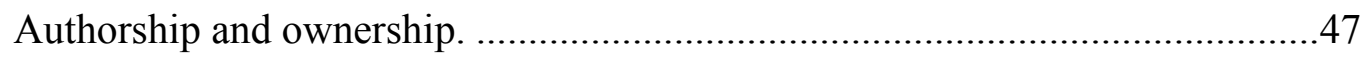

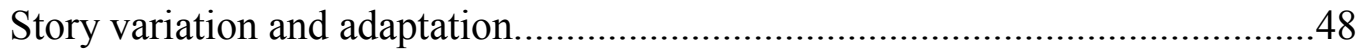

Character nature and role in culture. .........................................................49

The trickster in Native American culture ..................................................50

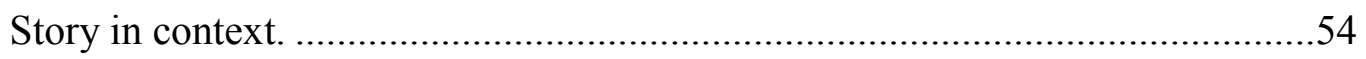

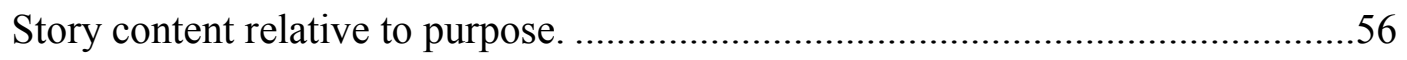

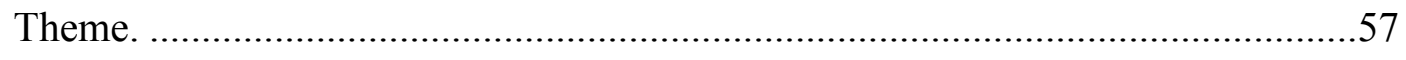

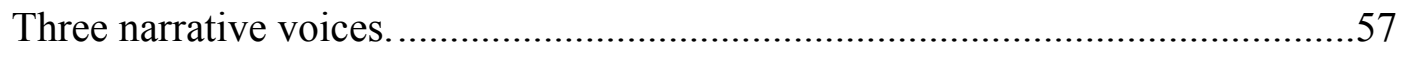

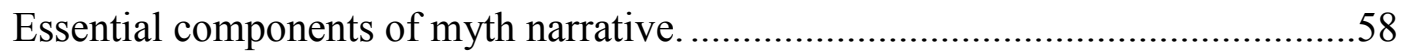


IKTOMI: A CHARACTER TRAITS ANALYSIS

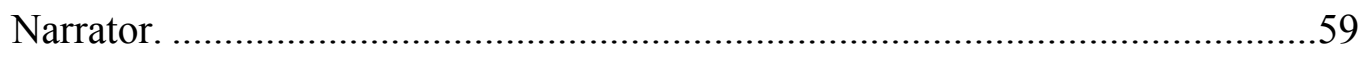

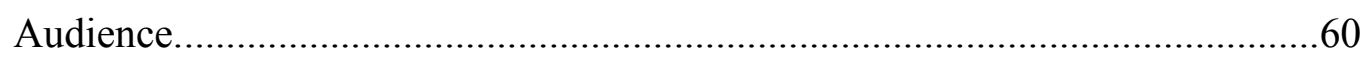

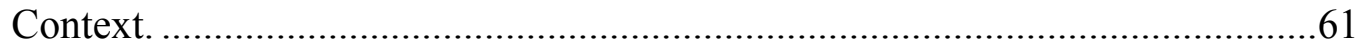

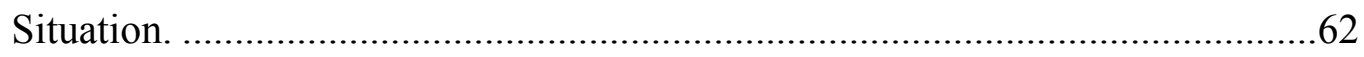

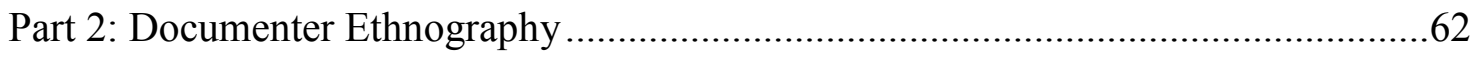

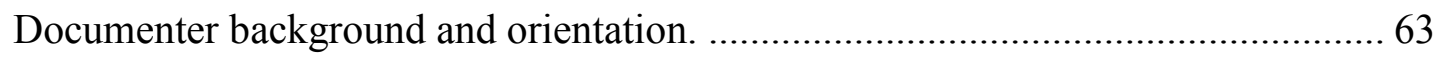

Ethnographers: Birth place, family life, and historical perspective......................63

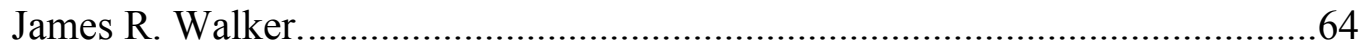

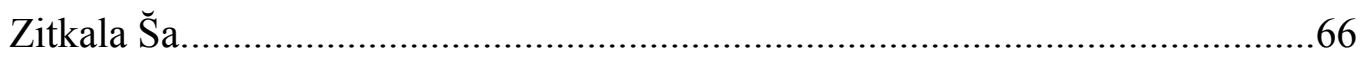

Ella C. Deloria..........................................................................................6

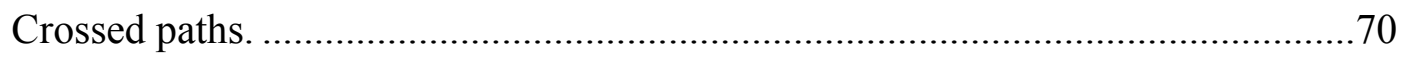

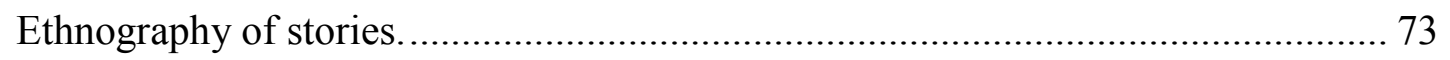

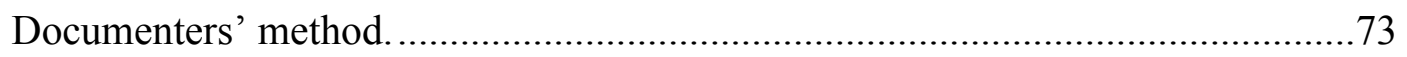

The collections: Sources and approach to recording stories. ..........................73

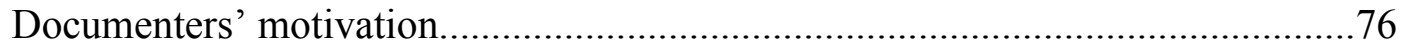

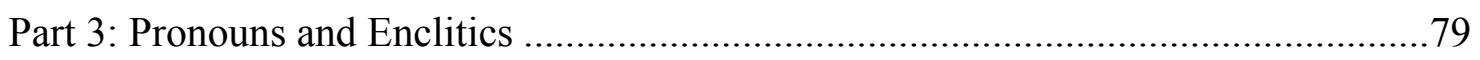

Dakota Pronoun Paradigm: Grammatical persons and number...........................81

Dakota enclitics as indicators of mode and gender....................................... 82

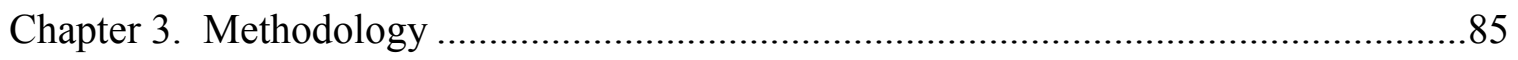

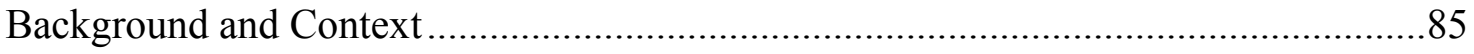

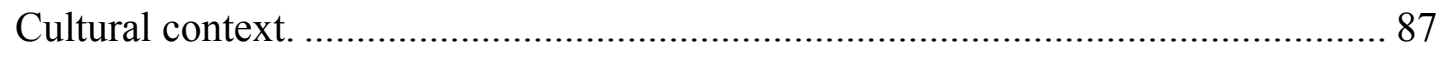

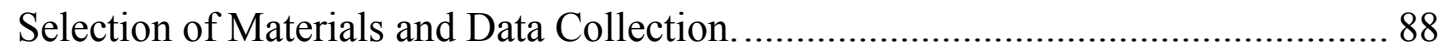




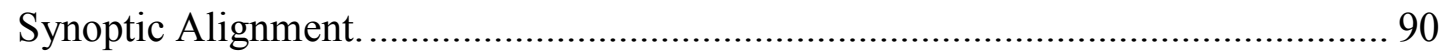

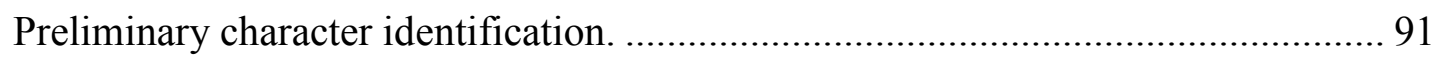

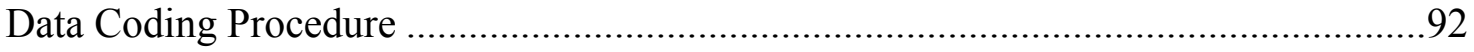

Phase 1: Character portrayal through traits. ................................................... 93

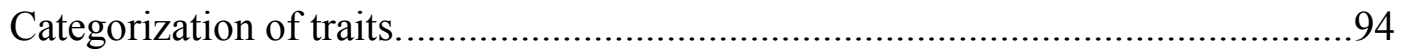

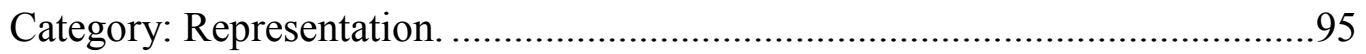

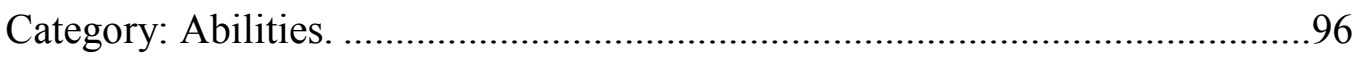

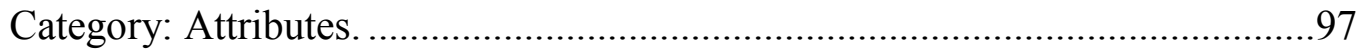

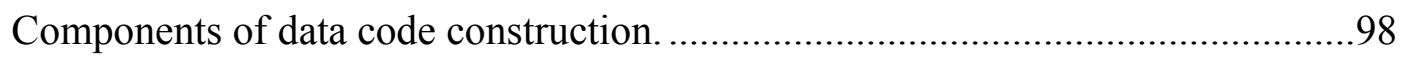

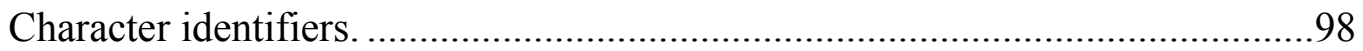

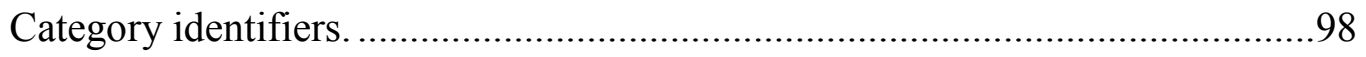

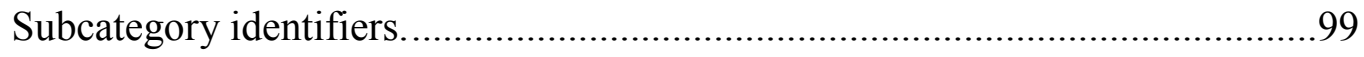

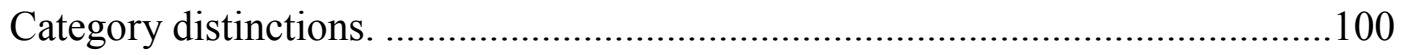

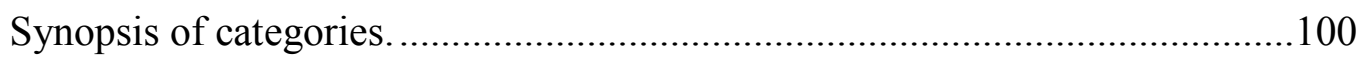

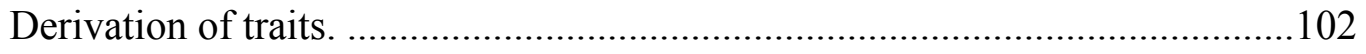

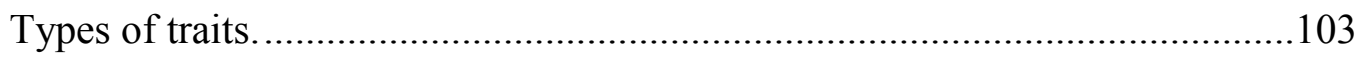

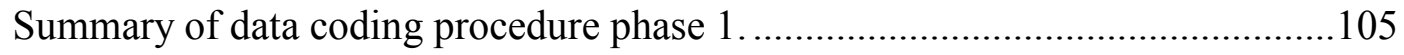

Phase 2: Polarity-code correspondences.................................................... 110

Derivation of Paired Polarity-Code Correspondences.....................................111

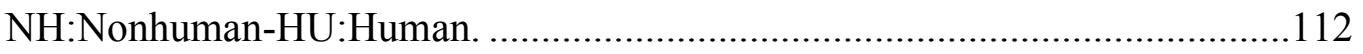

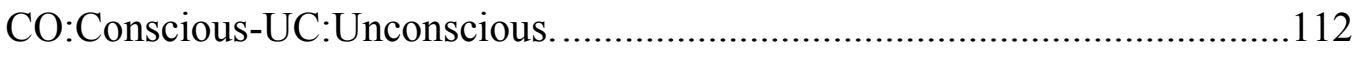

GP:Group Focus-IN:Individual Focus. ................................................. 113 
IKTOMI: A CHARACTER TRAITS ANALYSIS IX

IT:Intentional/Willful-PA:Passive/Receptive. ............................................114

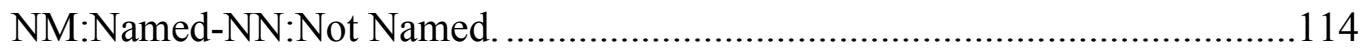

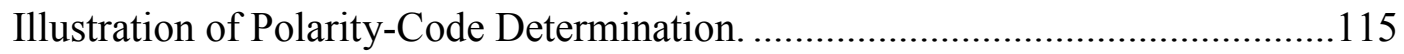

Phase 3: Pronoun usage. ............................................................................. 118

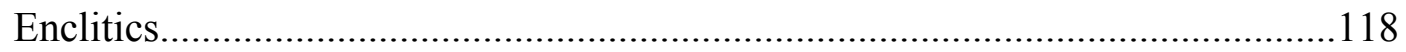

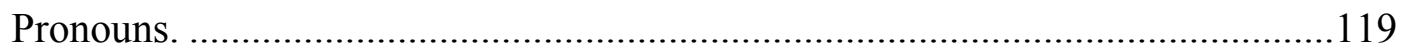

Documenter Motivation ................................................................................... 121

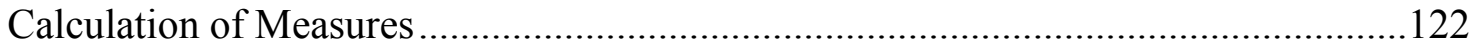

Character traits codes: Simple word counts of occurrences. .............................. 122

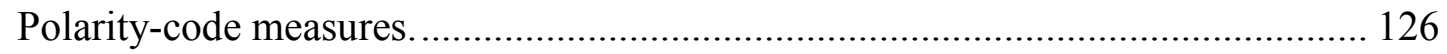

Modified Polarity-Code/Character-Code templates for individual characters. ....127

Compilation of polarity-code correspondences data......................................128

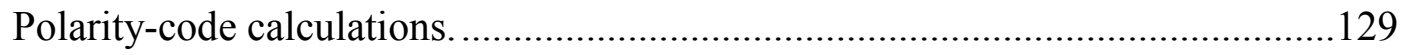

Weighted versus unweighted values. ....................................................130

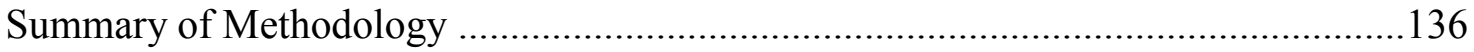

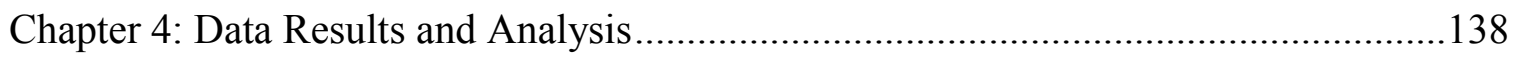

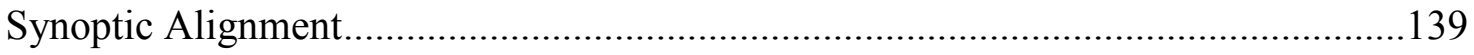

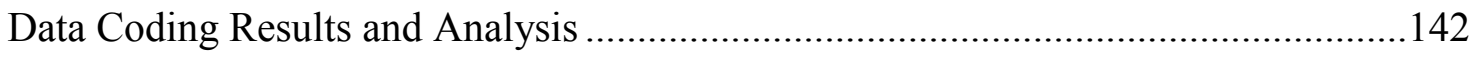

Phase 1:- Character traits codes and character portrayal. ................................ 143

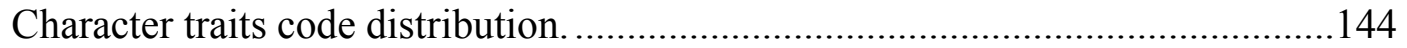

Traits code distribution among the potential characters...............................146

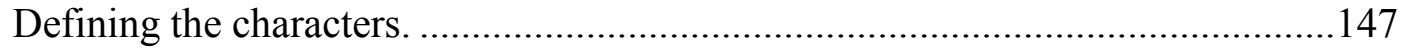


Code distribution and character roles. ......................................................... 150

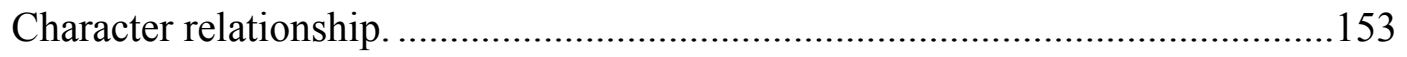

Character code distribution by version. ......................................................... 155

Summary of code distribution findings........................................................ 160

Phase 2: --Polarity-code correspondences..................................................... 163

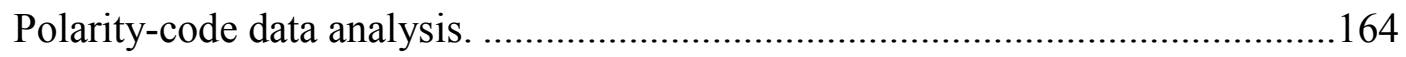

Overview of polarity-code correspondences results. ....................................165

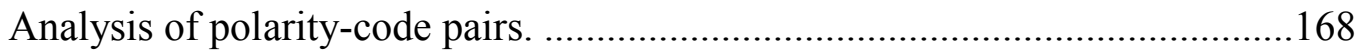

Summary of polarity-code correspondences: Character roles and relationships..175

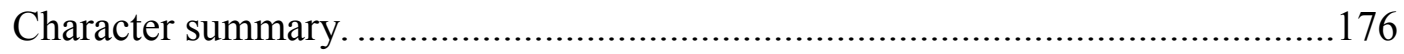

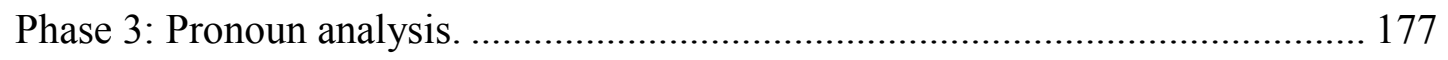

Enclitics as indicators of biological gender. ........................................... 178

Results, Analysis, and Findings of Pronoun Use...........................................181

Third-person-singular-masculine pronouns - he; him; himself; his. ...............182

Summary of findings: Third-person-singular-masculine pronouns. .................184

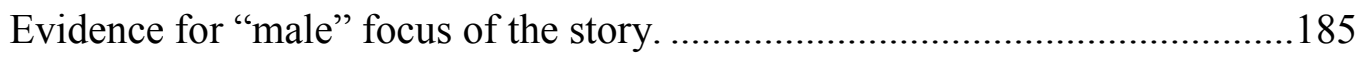

Other personal pronouns and determiners.................................................187

Summary of findings: Other personal pronouns and determiners...................187

Summary of pronoun analysis............................................................ 194

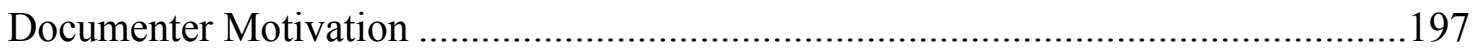

Documenters' role in the storytelling context. ............................................... 197

Effect of documenter's experience on the rendered story. ................................. 200 
Case for author preference for Ducks and Wolf/(ves) characters. ........................ 206

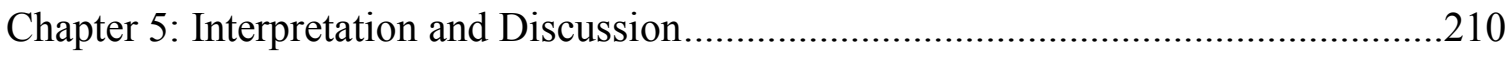

Overview of the Dakota Vision Quest. ............................................................210

Stages of the Vision Quest............................................................................ 212

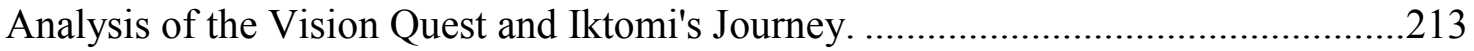

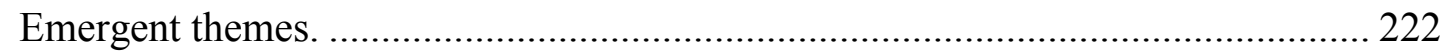

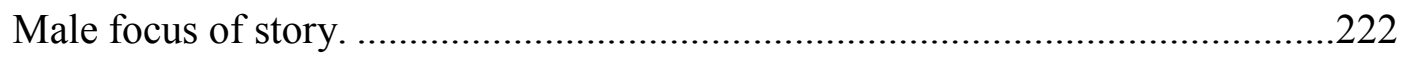

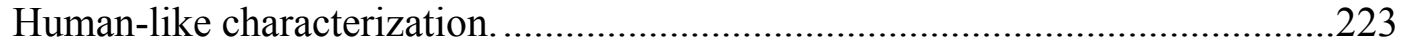

Duality in human experience. .................................................................224

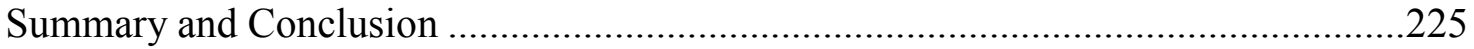

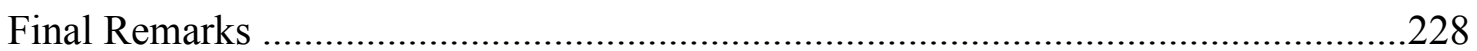

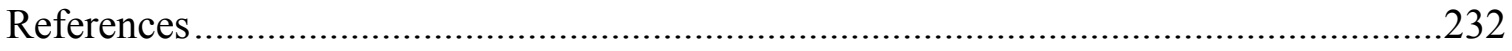

Appendix A: Iktomi Story Plot Summary: Correspondences between Episodic Sequence

of Events and Phases in Narrative Models ..............................................................239

Appendix B: Synoptic Alignment of Three Versions of Lakota Folk Myth Iktomi ........242

Appendix C: Character Traits Coding Template .....................................................252

Appendix D : Polarity-Code/Character-Code Correspondence Template .......................253 
IKTOMI: A CHARACTER TRAITS ANALYSIS

xii

\section{List of Tables}

\section{Chapter 3}

Table 1: Number of Occurrences of Traits Codes for Character Iktomi

Table 2: Occurrences of Character Traits Code Rp1 for All Characters and

Versions

Table 3: Iktomi's Character Traits Codes Corresponding to Polarity Code

NH:Nonhuman

Table 4: Unweighted Polarity-Code Correspondences Summary in Percentages .......132

Table 5: Weighted Polarity-Code Correspondences Summary .................................135

Table 6: Coded Traits Exhibited by Potential Characters in Composite for All

Versions

Table 7: Characters' Use of Enclitics in Deloria's Dakota Version of Iktomi Text.....179

Table 8: Character Distribution of Third Person Singular Masculine Pronouns .........183

Table 9: Other Personal Pronouns-Number of Occurrences as Used by Character ..189

Table 10: ..Other Indefinite Pronouns-Number of Occurrences as Used by

Character

\section{Chapter 5}

Table 11: Summary of Character Roles, Relationships, and Correspondence to Vision Quest. 


\section{List of Figures}

\section{Chapter 3}

Figure 1: Dakota System of Story Type Classification ...........................................8

Figure 2: Data Coding Process Flow Chart ...................................................... 108 


\section{Chapter 1. Iktomi: A Character Traits Analysis of a Dakota Cultural Myth}

In recent years, there has been a resurgence of interest in the Native American community among traditional and emerging storytellers to revitalize the practice of transmitting tribal lore through oral storytelling. A few tribal elders still serve as human repositories of the vanishing art form and archives of cultural history, and they work tirelessly in a concerted effort to pass on skills to younger Native American generations. Unfortunately, the remaining truly gifted storytellers are far too few in numbers for guaranteed success in preserving and passing on their skills. Furthermore, many Native American tribes have long since lost all of their knowledgeable historians of the tradition, and most of their emerging elders lack experience and training in earlier tribal practices.

The effects of assimilation and cultural oppression dating from the mid- $19^{\text {th }}$ century are in many cases irreversible: tribal families have been split up and dispersed; elders killed off; children trained to thwart their parents and guardians and alienated from their traditional beliefs and native languages. These factors, along with decades of Christian religious indoctrination, have obliterated or diluted the significance and meanings of Native beliefs and ceremonial rites once practiced and understood. In addition, the first peoples of North America have been driven from their ancestral lands, further severing their connection to their histories, while many of their descendants now live in urban settings disconnected from their culture, their people, and their stories.

When the American Indian Religious Freedom Act of 1978 lifted the prohibition against the Indians' right to practice their traditional ceremonies and cultural ways, it was already too late for many indigenous groups to recover. Some tribal memberships had 
been just too small to sustain themselves through the persecution, while others unluckily stood in the path of destruction as immigrating newcomers and profit seekers pressed their might over the land. Those tribes who managed to retain a few remnants of their culture, knowledge, and language were unorganized and disconnected from knowledge of and communication with other Indian groups. Other tribal groups and individuals fostered bitter resentment and distrust from the decades of loss and hardship, and they continued to keep their knowledge hidden, often even from their own relatives. Social dysfunctions caused by poverty, alcoholism, and drug addiction spread among the Native American populace so that, in many cases, those wisdom keepers who wished to share their knowledge had to first rehabilitate potential recipients by aggressively intervening against their substance abuse and maintaining constant vigilance to prevent backsliding.

Then there was the problem of carrying the message forward. Tribal members violently disagreed on the issue of sharing information and allowing participation by outsiders in their cultural practices. Leaders were divided about who had the right to tribal information, and hostile factions developed within the tribes in opposition to one another. Some Indians possessively refused outsider access to any type of cultural knowledge or customs while others maintained that no one could own their earth-based culture and, therefore, all people could share in it regardless of their race or background. A few open-minded medicine men courageously brought their teachings and ceremonies out from the reservations and welcomed Native and non-Native participants alike, however, that action drew severe criticism and harassment from their counterparts who 
remained protectively sequestered and hidden on Indian lands. The debates continue today.

Indigenous storytelling forums have emerged in the new millennium with renewed vigor and excitement not only for gifted tellers to showcase the traditional art form but also for them to pass on their cultural gift and encourage others to develop their own storytelling skills. However, while everyone is welcomed to attend performances and celebrations, some coalitions of storytellers restrict the mentoring opportunities to people of Native American descent and will not allow non-Natives to participate. Their protectionism is understandable and certainly to be respected in view of all else that has been done to and taken from their people. Still, while the willing and intelligent effort and support from non-Natives abide, they are held back from contributing significantly to the recovery process on account of skin color and blood quantum.

In that vein, scholarly contribution has served an invaluable purpose in restoring obscure and endangered cultural knowledge, as will be demonstrated by the citations presented in my review of the literature. In fact, the contributors of two of the versions of the myth analyzed in this study, along with their contemporaries, used their Western education and scholarship to advance our understanding of Dakota culture, practices, and values. Likewise, noted anthropologist Franz Boas, a Jewish German immigrant to the US, could make no claim whatsoever to Native American roots, yet he achieved an astounding magnitude of success in amassing tribal information and lore, while advancing the Indian cause for equal rights and justice. Drawing on his European experience, education, and roots, Boas recognized the flaws in the prevailing scientific 
paradigm and reformed methods to reflect a more humanistic approach to the study of human culture. Native American individuals have also turned to academia and research to advance their efforts of cultural revitalization, including Omaha anthropologist Francis La Flesche, Santee-Sioux physician and writer Charles A. Eastman, Lakota writer and historian George Bushotter, and Lakota linguist and educator Albert White Hat Sr.; the list goes on.

Despite these early contributions, little scholarly attention has been given to Native American stories since Hymes' and Tedlock's volley for poetic supremacy in the Native American folklore arena during the 1980s. So, why, some thirty years later, ought we now to be concerned again with Native American folk myths? Swann (1983) provided two reasons then, which still hold true today: “one, to overcome one's cultural isolation and narcissism by studying a civilization different in many important ways from our own; and two, in order to understand more of ourselves and our own civilization" (p. xv). Perhaps more than ever, we are in need of this wise counsel. Slotkin cautioned us, "A people unaware of its myths is likely to continue living by them though the world around that people may change and demand changes in their psychology, their world view, and their institutions" (as cited in Swann, 1983, p. xv). As the global expanse grows more accessible, we are ever more drawn into contact with and influenced by cultures different from our own. Additionally, many of us non-Native Americans have lost touch with our ancestral roots. A better understanding of the stories that emanate from the land we call home may help reorient and reconnect us to our land and our people — both Native and non-Native - and teach us how to cohabitate in an increasingly diverse world. 
My interest in Dakota culture took hold in the mid 1990s, when I attended a presentation on the Dakota culture, traditions, and prophecies. Since then, I have involved myself in the Dakota life way, participating in Dakota ceremonies, receiving teachings, and learning cultural practices by observing, listening, and following examples. As I expanded my experience and knowledge, my awareness and understanding also deepened, and I began to comprehend the multiplicity of layers embedded in the simplest teachings. I had also done some transcription work on interviews with tribal elders from various reservations scattered about the US, and I was fascinated by the stories they wove about their own lives as well as some of their accounts that explained cultural origins or past practices they remembered from long ago. I also sadly realized that none of those elders had been alive during the times they described in their tales and that much of the old lore of the tribal peoples had already slipped away with the elders who had passed on.

Although I enjoyed listening to the stories occasionally related orally by visiting elders, the few Native stories I found in print, at first, made little sense to me. Like other non-Natives and untrained readers, I found the material awkward and incomprehensible and I perused it superficially while making simplistic assumptions about its entertainment value. Eventually though, I realized these stories lingered as cultural relics, and I suspected they had more to offer than the face value at which I had initially taken them. I found multiple versions of some of the stories and compared them to try to make more sense out of them although reading them side by side presented a challenge and they still proved to be less than entertaining. Consequently, I set out to discover the deeper, more 
meaningful significance that I believed must lie embedded in the simplistic tales. Surely, I thought if the culture valued these stories so highly, there must indeed be inherent value beyond my trite first impressions.

On that quest, I set out to conduct an exploratory investigation to ascertain whether the story encodes and conveys cultural information and how it achieves that purpose. To accomplish my aim, I analyzed and compared three different English language versions of the same story in order to identify consistencies or inconsistencies among them and other significant aspects that might reveal cultural information that is not readily apparent through casual exposure. The three versions I used in the study include "Iktomi and the Ducks" from the collection in Old Indian Legends (Zitkala S̆a, 1901/1985, pp. 3-15), "Iktomi Tricks the Pheasants" from the collection in Dakota Texts (E. C. Deloria, 1932, pp. 19-25), and "Iktomi" from the collection in Lakota Myth (Walker, Jahner, \& SHSC, 1983, pp. 166-174). As a note of reference, throughout this paper, I refer to the story generically by the italicized name of the title character Iktomi; when referring to the title character, I omit the italics.

I chose this story of Iktomi for the study for its representation of a Dakota culture myth, for its feature of a trickster figure as its apparent main character, and because the three different versions markedly contrast with one another in terms of elocutionary style. The trickster is a familiar icon in tribal culture, and stories proliferate about his exploits, ostensibly for the purpose of teaching lessons of morality and cautious behavior through an entertaining venue to the younger members of the society. The story is easily recognizable for its entertaining style, though I strove to ascertain a deeper level of 
meaning from analysis to access its greater value as a mode of transmission of cultural information.

\section{Study Rationale, Purpose, and Research Questions}

While the aspects of storytelling highlighted in previous studies were used in attempts to ascertain significance of stylistic interest relative to specific texts, ultimately they have not informed the discipline with regard to cultural information that might be useful to individuals in the course of day-to-day life. My analysis of the text data in Iktomi has aimed to confirm the story as a tool of transmission of cultural values, traditions, and practices employed by the Dakota people in terms of readily usable information.

I employed a recontextualization model as a framework for this study, which takes into consideration the complementary interdependence of the facets of the story comprising the overview of the message along with the details of individual aspects of characterization. The model recognizes both a top-down as well as a bottom-up approach to the data woven together to show the construct of the story's fabric as a cohesive whole, into which, the cultural matrix can be infused. To unravel the constituent parts and understand the import of the story in more direct practical terms from the Native perspective, I posed the following questions, ranging in scope from the macro level of cultural purpose to the microcosm of practical use:

1. How does a story transmit cultural knowledge in practical terms?

2. How does a story reveal information that is useable on a practical day-to-day basis? 
3. What can we learn through the manner in which the characters are portrayed in the Dakota Iktomi story?

4. How can the characters and their relationships in these three versions of the Iktomi story give us insight into Dakota culture?

5. How can a detailed and systematic analysis of character representations, abilities, and attributes give us insight into the story's characters in the three versions of the Iktomi story, in Dakota storytelling, and in Dakota culture more broadly?

6. What analytic methods can give us insight into Native American storytelling techniques?

To facilitate the reader's ability to follow the information discussed throughout this study, I offer here a skeletal synopsis of the Iktomi story:

The trickster Iktomi wanders aimlessly along until he comes upon a group of ducks. He thinks to himself that they would be good to eat so he devises a scheme to catch a few. He succeeds and sets them to roast in a fire. While he waits, he becomes distracted by some noisy trees overhead. He climbs up one of the trees to separate the branches from rubbing against each other though instead, he gets caught in them. While trapped in the treetop, Iktomi sees wolves in the distance so he starts shouting, which attracts their attention. The wolves hurry over, find the roasting ducks, and eat them all up so Iktomi gets none for himself.

Dakota stories are told in sequences of individual segments or episodes, and certain segments can be added or omitted to lengthen or shorten a story for any occasion. The episodes may thus be told singly or in any combination. Because of the anecdotal nature of the episodes and because the tales do not assume a strict temporal or spatial ordering, the storyteller has the flexibility to present them in any order, so long as the 
linearity in the consecutive sequence of events within the individual episodes is maintained (Kimball, 1993, p. 4). For instance, in this Iktomi tale, the character Iktomi must first kill the ducks before the wolves can come along and eat them; the event sequence cannot logically occur the other way around. Otherwise, Iktomi's encounter with the ducks takes place independently of his encounter with the wolves. I note, however, that Kroeber warned against attempting to impose a Western restriction on the linearity of logical, serial continuity between episodes strung together in a single telling. He intimated the ordering of sequences "may often be more complex" from the Native perspective than Westerners are trained to understand and warrants more study before we can confidently assume what we think we know about logical sequence as it pertains to Native storytelling (1998, p, 237).

Various component segments in which Iktomi encounters the different characters comprise the three different versions of the Iktomi story used in this study; each version maintains the general sequence timing consistently with the others, although some variation exists from one version to another with regard to the details of the encounters.

My study begins in Chapter 2 with a review of the literature and background information to put the Iktomi story in context from a variety of perspectives, followed by a detailed methodology in Chapter 3 delineating my data coding and analysis processes. Then, in Chapter 4, I present my findings and discussion of the results. I conclude the study in Chapter 5 with my interpretation, tying the results back to my original premise, and lastly attach a list of references for works cited and appendices. 
I divided the literature review in Chapter 2 into three parts: the first part covers social theoretical aspects of the study and scholarly approaches to Native American folk myths that have had some bearing on the outcome of the renditions of the texts under investigation; the second part deals with the documenters with regard to their personal history, motivation, and collection of story materials; in Part 3, I present information about grammatical markers of sex and mode incorporated in the Dakota language that emerged as relevant to part of my analysis.

In Chapter 3, I describe the methodology I used to code and analyze the text data along with my rationale for choosing my particular approach. First, background information orients the reader to the story's place in the Dakota culture and with respect to the different versions used in the study. Next, I detail my treatment of the texts which included synoptic alignment, character identification, data coding and categorization of character traits, determination of correspondences among coded traits in the data, and examination of the special attention given to the use of pronouns in the text. Then, I describe my consideration of documenter motivation with regard to its effect on the outcome of the individual versions and its impact on the data. The chapter concludes with explanations of the specific procedures I used to calculate measures for analysis.

My review of the literature proved vastly informative and wide-reaching in scope; yet, I remained unsatisfied that earlier studies succeeded in unlocking the likely significance of the Native American story, which has been commonly purported to be a practical tool for transmission of cultural information and as an educational, guidance, and counseling instrument. Instead, as I discuss in Chapter 2, I found admirable 
scholarly work that resulted in many insights into the performance nature of storytelling, stylistic expression, and broad psychoanalytical theories of overarching theme; however, there still seemed to be lacking the element of practical usability that we have been promised. I maintain that the more critical element of practicality exists in the Native American folk myth, and I based my study on the objective of discovering its presence in the story Iktomi. I now proceed with backgrounding my study in Chapter 2 with a review of the literature. 


\section{Chapter 2. Background and Literature Review}

In the first part of the literature review, I present social theoretical aspects and scholarly approaches to Native American folk myths which I found relevant to the study. Background information from a social theoretical standpoint outlines how scholars have traditionally viewed Native American folk myths and stories in general and the role the stories played in tribal society. Then, my discussion of the scientific paradigms operating in the world during the period of collection acquaints the reader with the approach and methods that were typically used at that time to collect, document, and study cultural artifacts, including stories and language materials, with brief comment about Boas's significant contribution to the study of culture. Next, a description of the political climate in the United States leading up to and during the period of collection and publication of the three versions of the story illuminates the emotional impact of events on the lives of the documenters that may have influenced the final renderings of their respective versions of the story.

My discussion of scholarly approaches to Native American folk myths follows, beginning with definitions of certain terms used to talk about narrative myths. Then I address the concept of symbol as used in the context of tribal myth, and myth as performed verbal art. I additionally mention the styles and techniques used to document the texts used in this study. The discussion of scholarly approaches also includes cognitive views of story content including models and frameworks used to organize the text into themes and stages of progression, and I demonstrate structural continuity among the three versions. I contrast the pioneering work of Hymes and Tedlock in the study of 
Native American storytelling and discuss how the Iktomi story fits with their propositions. Then, I address aspects, content, and themes that emerge from the stories and incorporate Native views on authorship, story variation, the cultural role of the story, role of the trickster figure, contextual relevance, and story content. The section concludes with an elaboration of the essential components of myth narrative from the Native American perspective.

In Part 2 of the literature review, I background the lives of the three documenters in order to illuminate the environments, experiences, and influences that helped shape their attitudes and motivations. I describe their home life, education and training, professional work, aspirations, and accomplishments, all set against a sociopolitical backdrop. Then I draw the connections of people and place that linked the context of their experiences highlighting their mutuality of purpose and common sources for the stories. Finally, I give a detailed account of the method and process each of the documenters used to collect, document, and translate their texts and how their respective versions came to the form I used here. I conclude this section of the literature review by tying together the facts gleaned from the personal lives and experiences of the documenters, historical events, and the social context of the time and assimilate them into my own perspective, which I inferred from the content.

Finally, the third part of the literature review concerns the Dakota pronoun paradigm as it relates to English and Dakota markers of biological sex, which I show to be relevant to my discussion about the characters, their relationships, and their purpose in the story. 
To contextualize the discussion material for the reader, a brief background about the people from whom the Iktomi story comes to us provides a bit of perspective about who they are and where they live today.

These versions of the story were related and recorded among the Sioux Indians of the American Great Plains who refer to themselves collectively as the Dakota, meaning simply "the people." The tribe is subdivided into three groups, which speak separate dialects of the Dakota language: Nakota, Dakota, and Lakota.

In this paper, I primarily use the collective term Dakota to refer to the people of the culture from which this Iktomi story was recorded, although occasionally I use it interchangeably with Lakota, Sioux, Native, Native American, or Indian, depending on the context. I also reference the language generically and interchangeably as Lakota or Dakota, since the stories were related by tellers of varied tribal background who spoke either of the varieties. The more common term Sioux technically refers to a component of the greater collection of languages comprising the Siouan language family and so dialectal reference is more appropriate in this case.

\section{Part 1: Social Theory and Scholarly Approaches to Native American Myths}

\section{Social theory.}

This first section addresses traditional views of Native American folk myths and stories and the story's role in tribal society, prevailing scientific paradigms, the influence of Boas on studies of culture, and the US political climate affecting emotional impact and influence on the final renderings of the texts. 
Dakota myths are available for study today thanks to the work of important field researchers who collected and compiled materials from indigenous people during the 1800s. Among the early ethnographers, James Owen Dorsey collected volumes of Dakota stories, while Franz Boas and his team of anthropologists at Columbia University also amassed a large amount of material from many different tribal groups, much of which remains untouched in private collections and historical archives. More recent scholars have revisited documented texts to prepare them for publication or conduct ongoing research. For example, Raymond DeMallie translated and interpreted work on numerous texts resulting in several informative books pertaining to Lakota culture and religion (e.g., Black, Neihardt, \& DeMallie, 2008; DeMallie, 1975; DeMallie \& Ortiz, 1994, DeMallie \& Parks, 1987; DeMallie, Sturtevant, \& Smithsonian Institution, 2001; Mooney \& DeMallie, 1991; Neihardt, Black, \& DeMallie, 1985; Poole \& DeMallie, 1988; Walker, DeMallie, \& Jahner, 1980). Others have supplied major contributions through their work with other tribes, providing a framework for investigation and comparison, such as Paul Radin's unparalleled work concerning the Winnebago trickster, Dell Hymes' groundbreaking work with the Chinookan tribal lore along the Columbia Basin (Roemer, 1983, p. 40; cf. Brown et al., 2004; Deloria, 1999, p. 19; D. Hymes, 1980, 1982; Hymes, 1955, 1993; Hymes \& Simpson, 1976; Radin, 1953, 1957, 1972; Radin, Kerényi, \& Jung, 1956a), and Ruth Bunzel's early efforts (cf. Bunzel, 1932a, 1932b, 1932c, 1974) followed by Dennis Tedlock's later work with the Zuni Pueblo tales of New Mexico (cf. Tedlock, 1968, 1999; Tedlock, Peynetsa, \& Sanchez, 1972), to name 
just a few. In the next section, I background the nature and role of myth and folklore in the cultural context.

\section{Myth and folklore in Dakota culture.}

Folk stories and myths hold an important place in tribal culture; however, despite the amount of information available and the extent of research that has involved tribal stories and storytelling, they remain somewhat of a mystery. I attempt to join the effort in unraveling the enigma — what the Dakota would term "wakaך" or "the unknowable and powerful essence" - of the Native American myth by undertaking this study of the Dakota story Iktomi. In the following section I present some of the views held by scholars about Native American storytelling, the role and value of stories held by the society, and how cultural myths relate to adults and children.

\section{Social role of stories: Perception and value.}

Aside from entertainment, the underlying purpose of folk stories is to maintain social order in its relationship to nature (D. Hymes, 1975, p. 358) as well as to preserve cultural traditions and practices. Native American stories and myths had rarely been taken seriously by non-Native observers who likened them to children's stories and fairy tales that were told for amusement in Western culture (Roemer, 1983). However, scholars more recently have made a serious attempt to resituate the Native narrative in a place of respect among the literary collections from other cultures around the globe (cf. Clements, 1996; Roemer, 1983; Sherzer \& Woodbury, 1987). To the untrained Western ear, tribal myths come across as fanciful, often very simplistic or hard to follow, and lacking in serious plot construction, while their characters seem cartoonish or perform 
impossible acts. From the Native point of view, a person's ability to laugh at himself or herself while conveying important social lessons argues one of myth's greatest strengths and purposes. For example, the actions and adventures of the mythical trickster character serve to poke fun at life's circumstances, errors of judgment, and one's foolish actions and motivations in order to illuminate behavior viewed as detrimental to the tribe or individual (Horne, 1999, pp. 127-152).

Ackerman believed the artistic and interpretive value of Native folklore directly correlated with the contentment and well-being of individuals and the healthy functioning of the society as a whole (cited in D. Hymes, 1975, p. 346). In other words, stories enabled storytellers and their audiences within a society to share in the experience of addressing particular social concerns through the non-threatening form of entertainment and distancing themselves through character substitution. The more culturally relevant the story proved to the people, the greater importance it carried for them; consequently, they would expend more effort to preserve it and pass it on.

As the central focus of the myth narrative, characters "represent different value orientations in conflict with each other and/or the environment" and generate emotional response in the audience who identifies with them (Fisher, W., 1987, pp. 47, 161). The audience members weigh the characters' actions against their own values and selfperception and either accept or reject them based on the characters' reliability, trustworthiness, and believability. As Aristotle observed long ago, individuals naturally tend "to prefer what they perceive to be true and just" (1954, as cited in Fisher, W., 1984, p. 67); that is, people naturally reflect how they themselves wish to be perceived. In his 
introduction to Narrative and Social Control, Mumby (1993) connected this aspect of human nature to the narrative: "we are never neutral, dispassionate observers of behavior but are always heavily implicated in the construction of the narratives" (p. 4). In effect, we act as co-constructors of the stories as they are transmitted and passed along from storytellers to audiences and other storytellers through the way we respond, interact, or modify our behavior (p. 5).

\section{Myth and socialization of children.}

Tales of myth were and still are used effectively to indoctrinate children into the social culture of tribal society. Brian Sutton-Smith's (1986) study of language disorders suggested that as cognition and memory in young children relate to their own experience, stories correlating to cultural experience strongly influence cognitive development (p. 2; cf. also Brice Heath, 1986, pp. 84-85). He found that children under three years of age are able to perceive identifiable traits of story sound, structure and meaning, relationship of metaphor to real life, characterization separate from the storyteller, analogy to personal experience, and vocal variations in intonation and pauses (pp. 5-6). As children approach seven years of age, they begin to understand plot, character roles, timing and sequence. Between ages eight and twelve years, children associate plot formulation in conjunction with some human conflict and expect a resolution (p. 9).

Although Sutton-Smith's study related to personal narrative, his findings apply to the role of mythic narrative as well. In indigenous communities, parents often told stories with adult themes to children with the understanding that they could grasp a level of understanding about its message. The stories were often repeated with the expectation 
that depth of understanding and insight would develop and increase as the child grew older and more mature. Yet, Swann (1983) maintained unequivocally, however, that cultural stories were in reality the business of adults (p. xiv). Lincoln (1983) elaborated, citing stories as collectively reflective and useful in opening the way for resolution of conflict and social discord by serving as a release valve for tension, fear, and emotionally difficult situations.

To illustrate this concept, Roemer (1983) suggested that during the historical period in which the Iktomi versions were collected, sharing familiar tribal stories helped the Indians endure and describe the shocking cultural changes they experienced (p. 42; also Kroeber, 1998). For example, like the trusting ducks in Iktomi, the Dakota people extended hospitality to the strangers who, at first, appeared to be only passing through their lands. However, when they eventually realized the intruders intended to kill them off, both the ducks and the Dakota scattered and only a few escaped with their lives. Another illustration depicts Iktomi sitting by helplessly while he watches a marauding wolf consume his whole cache of food; similarly, White settlers looted Indian camps and villages, taking or destroying all their provisions and leaving the Indian people destitute. Roemer's and Kroeber's observation is particularly relevant to Zitkala S̆a's motivation for writing her collections, which I elaborate on in Part 2 later in this chapter and revisit in Chapter 4.

\section{Western perspective: Timeline of Western thought and advent of the Boasian Approach.}

A conceptualization of the prevailing thought paradigms in the world at the time the versions of Iktomi were collected is useful to help understand how awareness and 
perceptions of Native storytelling emerged and changed to affect the distinct presentations of each of the versions.

The period of change and oppression for the American Indian spanned about one hundred and fifty years from the 1830 s to the latter decades of the $20^{\text {th }}$ century. The most critical influences emerged with the Age of Positivism, stretching from the 1860s to the end of World War I. Characterized by an emphasis on scientific application in everyday life during this time, reason became the vehicle for understanding and making improvements in world conditions. In Europe, Ferdinand de Saussure pioneered a new body of thought centered on the human sciences, whereby he applied scientific reasoning in a structuralist approach, viewing the whole system in terms of its interrelated parts. Concurrently, the Russian Ivan Pavlov conducted experiments in behaviorism, creating a foundation for observing behavior patterns and connecting thought with action (i.e., response). Scientists in other social scientific fields shortly followed on the work of these two men. In particular, on the North American continent, German-born Jewish anthropologist Franz Boas became very influential with the development of his refined scientific method of inquiry into the study of human cultures. His disciplined approach involved collecting data by observing people in their own habitat and using them as empirical evidence to formulate and test hypotheses.

The Boas Era, or the Era of Liberal Reform, marked a dramatic shift in the scientific paradigm and spanned his entire lifetime from 1890 to about 1945 (cf. Wolf cited in Mintz, 1985, pp. 9-10). Boas distinguished himself as the forerunner of modern anthropology, making his major impact on the discipline during his tenure at Columbia 
University from which he pursued his lifelong humanitarian goal: seeking to promote racial equality and instituting a more disciplined approach to science (Mintz, 1985, p. 9).

\section{Sioux history in brief.}

Along with the scientific paradigm that influenced how Native American stories have been handled, the social and political climate affecting the lives of the Indian people must also be considered to contextualize the environment from which the stories were exhumed. It must be remembered that the three documenters lived and participated in the activities of current events in the nation during a time of upheaval and strife perpetrated against many different minority groups, as I will describe in the next section. The Indian tribes represented a unique barrier against the promise of the American Dream of prosperity in the Land of Plenty, and the atrocities they suffered consequently affected the attitudes of all those who participated in telling, recording, and translating the stories. In effect, a general sense of despair had descended over the survivors of the destruction of Native American communities and recolored their perspectives with a dark palette. A brief summary of events in the decades spanning the lives and work of the documenters follows to show how the sociopolitical climate may have influenced their motivations and methods for recording the stories.

US Indian Policy and Reimaging of Native Americans.

I condensed and reassembled the following information from several general information books written by and about Indians and historical references, consultation with maps, and cross-checking of events, places, and dates through various websites on 
the internet. ${ }^{1}$ Much has been written about the bleak history of the American Indian and has come into widespread general public awareness. I reiterate a few of the details here to assist the reader in contextualizing the conditions under which the documenters of the Iktomi versions were born, worked, and lived their lives.

Throughout the $19^{\text {th }}$ and early $20^{\text {th }}$ centuries, two influences helped shape the prevailing perception and attitude toward the Indian people among European immigrants advancing over the American continent. The first manifested itself as a remolding of Indian identity by the growing White population and a quest to obliterate the Native's self-perception by creating and superimposing the new identity over it. Influential proponents of Western progression supported and encouraged writers of the period to portray the Native inhabitants as one-dimensional violent savages (Schöler, 1981)—wild, ignorant, and not-quite-human creatures. The second influence manifested itself as hostile action against the Indian people via the US government policy of assimilation and eradication of their language and culture. Native occupants stood in the way of the new Americans, who lusted greedily after land, money, and power; hence, there was a complicit government campaign to degrade and deprive the Indian of human status and rights. By denying the humanity of the Indian, the US government found justification for carrying out its policy of extermination and brutality over the next several decades.

${ }^{1}$ Cf. Deloria, V. (1999); DeMallie (1993); Karolevitz (1975); Lincoln (1983); Marshall (2001); Native American Citizenship. (n.d.); Native American People/Tribes: Great Sioux Nation (2005); Olson (1965); Picotte \& Pavich (1992); Robinson (1966); Schöler (1981); Sioux History Timeline (2007); Utley (1984); Walker et al. (1983); Young Bear \& Theisz (1994); and Zinn (1980). 
State of the US.

As the $19^{\text {th }}$ century unfurled, the young country hardly existed in a state of peace and prosperity, and the Indians represented only one group that suffered. However, they served conveniently as scapegoat and distraction from the many hardships and cruelties experienced by competing factions and presented an easy target for escalating atrocities. The US carried out a duplicitous campaign against the Indians while feigning cooperation, compromise, and conciliation. Beginning in 1830 and lasting a decade, the Indian Removal campaign etched the Cherokee Trail of Tears into painful memory; with it, the first wave of smallpox epidemics swept into the Great Plains to decimate the tribal population (Karolevitz, 1975, p. 34).

US Indian conflict.

The California Gold Rush, which boomed in1849, brought even more movement through Indian territories; eventually, the US entered into a series of treaties with the Dakota people to secure safe passage through Dakota lands and maintain peace between the Whites and Indians. The first Treaty of Fort Laramie in 1851 enabled White settlers to travel unmolested through Sioux Territory while recognizing Indian ownership of the vast territory (Karolevitz, 1975, pp. 40-41). The effort was made to appease the Dakota people and guarantee their autonomy and stewardship over their own territory; however, a few years later the US reneged on its agreement and carved the states of Minnesota and Iowa out of the tribally owned land (Robinson, 1966, pp. 98-99).

In 1862, under President Lincoln, as Southern states seceded from the union over the slavery debate, the Homestead Act precipitated another mass migration to unclaimed 
territory west of the Appalachians (Karolevitz, 1975, p. 63; Robinson, 1966, p. 148).

This time, small farmers displaced by larger profitable southern plantations, in turn, displaced the Sioux from their own homelands in Wisconsin and Minnesota, driving a deeper wedge between the Sioux and their Chippewa rivals, who competed for the same space. The increasing pressure on the Indians erupted in the Minnesota Massacre and Great Sioux Uprising, a rebellion of Indians against Whites which left hundreds of innocent men, women, and children dead on both sides (Karolevitz, 1975, pp. 63-64; Robinson, 1966, p. 128). As the US engaged in Civil War back East, hostilities were more and more redirected toward the Indians farther west in the form of unprovoked surprise attacks and unchecked brutality. For example, in the 1864 Sand Creek Massacre in Colorado Territory, the uncontrolled military sportingly destroyed an entire village of innocent men, women, and children simply because they were Indian (Deloria, 1999, p. 307). With the close of the Civil War, the Charter for the North Pacific Railroad allowed the confiscation of even more Indian lands protected by treaty to gain safe passage to transport goods and people through Indian Territory (Robinson, 1966, pp. 125, 127). The invasions exhausted the Indians' congeniality, eventuating Red Cloud's retaliatory war to drive the Whites out of their sacred Black Hills in today's South Dakota and Bozeman territory in Montana (Olson, 1965). The US again sought conciliation by offering another treaty - the 1868 Treaty of Fort Laramie - once more promising to stay out of Indian lands. This treaty resulted in the establishment and protected rights of the Indians of the Great Sioux Nation-land stretching between Wisconsin and the Big Horn Mountains in Wyoming and Montana and from Canada to the North Republican River in 
Kansas (Karolevitz, 1975, pp. 100-102). Ironically, the US concurrently passed the $15^{\text {th }}$ Amendment, which granted voting rights to Blacks but deliberately excluded Indians from citizenship and their right to vote. In effect, the US once again failed to recognize in Indians their inherent quality of humanness and the right to human dignity (Zinn, 1980, pp. 96, 198).

By the 1870s, the US government had stepped up its oppression of the Indians and ceased to recognize tribal autonomy. Indians had relocated to reservations and depended on Government Issue in the form of food and goods; however, control over the Indian agencies which handled the commodities had been turned over from the military to Christian missions who misappropriated them, and the Indians began to suffer the effects of deprivation of essential provisions. As the missions took over, Indian boarding schools proliferated, too, promoting assimilation and civilization of the Natives through Christian indoctrination and education (Utley, 1984, pp. 127-133).

Finally, as the US celebrated 100 years of independence from English tyranny, the Indians' struggle for their own liberation from oppressive invaders culminated in the famous Battle of the Little Big Horn in Montana Territory. General George Custer and the $7^{\text {th }}$ US Cavalry met defeat and disgrace at the hands of the Great Sioux chiefs, Sitting Bull, Gall and Crazy Horse leading a massive gathering of Sioux warriors (DeMallie, 1993, pp. 516-517; Karolevitz, 1975, pp. 117-118, Marshall, 2001, pp. 10, 215).

Indian victory was short-lived, however, and the US folly and embarrassment spurred renewed determination to subdue and annihilate the Indian obstacle. The following decade saw the final collapse of the traditional Indian way of life as the 
remaining Sioux Chiefs Gall and Sitting Bull surrendered, the US government outlawed Sioux customs and ceremonies, and the wholesale slaughter of the buffalo on which the Indians depended for life, food, shelter, clothing, and tools finally sealed their fate, ultimately starving them onto the reservations (Lincoln, 1983, p. 5).

Scattering of the tribes continued as the Dawes General Allotment Act of 1887 parceled out Indian reservation lands which had been communally-owned to individuals in a deliberate attempt to disintegrate tribal unity. The Dawes Act along with the Sioux Land Commission of 1889 effectively cut the Great Sioux Reservation by half, then broke up the remaining lands into smaller reservations and further parceled out allotments to individuals and families (Marshall, 2001, p. 218; Picotte \& Pavich, 1992, p. v; Robinson, 1966, pp. 199-203; Walker et al., 1983, p. 1; Young Bear \& Theisz, 1994, p. xxix). The final years of the Great Sioux Nation culminated with the 1890 murder of Chief Sitting Bull over the Ghost Dance fiasco and the massacre of Big Foot's band at Wounded Knee in South Dakota (Young Bear \& Theisz, 1994, p. xxix). Thus relegated to a life of confinement, abuse, and starvation, the Sioux people were left depressed and without hope: most of their revered leaders were dead, their lands had been confiscated, and a self-proclaimed superior society denigrated their once proud people.

I conclude my discussion of the sociopolitical conditions serving as a contextual backdrop for the stories used in the study and now turn to a presentation of the critical aspects of storytelling as they relate to my purpose. 
IKTOMI: A CHARACTER TRAITS ANALYSIS

\section{Scholarly approaches to Native American folk myths.}

In this section, I will put forward a discussion of scholarly approaches to the study of Native American folk myths in two segments. In the first segment, I explain specific concepts used to talk about folklore and folk myths including a description of the narrative paradigm from a Western perspective and how Native American myth fits with it. Next, I explain the concept of the symbol as it is used in myth and then, myth as a form of performed verbal art. Finally, I address the conceptual framework of the documentation that was used for these versions, followed by a discussion of the documenters' styles, including shortcomings and criticisms made of the techniques they used to record linguistic data.

In the second segment, I place the Iktomi story in organizational context with regard to cognitive approaches to story content and include my conception of how the story fits according to similar models devised by earlier researchers. This section includes discourse concerning the two major contributors to the study of Native American storytelling, Dell Hymes and Dennis Tedlock, where I contrast their different approaches, discuss other scholars' reactions to them, and include my own perspective regarding their relevance to Iktomi. Next, I discuss the characteristics of myth, relating them specifically to their expression within the Dakota culture, e.g., the concept of authorship, variation and adaptation, characters and their cultural roles, elaboration on the trickster figure, contextual relativity of stories, and story content and theme. I briefly mention the three narrative voices and show how stories directly apply to everyday life; 
and finally, I outline the essential components constituting Native American myth narrative.

\section{Discussion of myth and folklore.}

To aid in understanding the story used in this study, I think it is appropriate to define the terms scholars use to classify its type in the context of Western folk literature and of Native American storytelling. This section provides explanations for those concepts I found relevant to this analysis.

Although the investigative fields of science have not been able to agree definitively on what actually constitutes myth (Kroeber, 1998, p. 62; Gill, 1987, p. 157), from her perspective within the Dakota culture, E. C. Deloria (1932) places the story of Iktomi among the ohuykakay tales of the Dakota--mythological accounts of past fictional events artistically constructed to amuse or entertain an audience (p. ix). Thus, like their Western counterparts, the mythological stories of the ohuykakan have filtered down through the generations by way of cultural storytellers and have merged into the collective memory as part of the folklore of the Dakota people.

\section{Narrative paradigm.}

The Dakota Iktomi story parallels W. Fisher's narrative paradigm, which he termed a universal metacode of "symbolic actions — words and/or deeds — that have sequence and meaning for those who live, create, or interpret them" (1984, p. 2). Narrative in these terms includes an experiencer who becomes the creator and teller as well as a hearer who interprets and then becomes an experiencer. Thus, the process is cyclic and all participants' roles are interrelated, an important point that I return to in 
Chapters 4 and 5. Real experience communicated in symbols provides the inherent value of narrative, ordering the society, transmitting shared cultural information (White, 1980, as cited in Fisher, W., 1987, p. 65), and reinforcing "ways of living in common" (Fisher, W., 1984, p. 6; cf. also Burke, 1957, Fisher, W., 1987, D. Hymes, 1980, Turner, 1980).

While W. Fisher spoke of personal narrative of individuals, his conceptualization extends to impersonal stories which hold relevance and significance for the collective community of individuals. Campbell professed, "the most compelling, persuasive stories are mythic in form, stories reflective of "public dreams" that give meaning and significance to life" (as cited in Fisher, W., 1987, p. 76). Again, Campbell repeated the collective theme in his reference to public dreams, which implies shared experience, hopes, and values. While narrative may originate in the personal experience of one individual, as a human universal, once told, it becomes generally available to anyone who might hear or read the narrative regardless of their background or orientation, and all the participants in the storytelling event become co-creators of the narrative.

Valued narrative also maintains consistent form and progression while perpetuating high moral standards, as well as a thread of truth perceivable to the audience. Despite the fantasy of myth, the narrative must be believable on some level and conform to the values held by the society to be accepted as a cultural truth that meets a standard of acceptability (Fisher, W., 1984, p. 16). The Dakota myth must meet these standards in order to fulfill its function as an authentic vehicle of transmission of essential cultural information. 
Narrative symbol in myth.

I based my analysis of Iktomi primarily on its symbolism, that is, the portrayal of the characters and what they, their actions, and their interactions with other characters signify. Various social scientists, including linguists, have used the term "symbol" interchangeably with the related concepts of "sign" and "signifier" in different, sometimes contradictory contexts. Seeking to clear up the inconsistencies and confusion, Arrivé analyzed the theories of Saussure, Hjelmslev, and Freud, among others, to arrive at a simplified outline of traits that characterize the linguistic symbol based on how it is used in legend (i.e., myth; in Arrivé, Coquet, \& Leader, 1992; for more discussion of symbol and representation in myth, cf. Bakhtin, Holquist, \& Emerson, 1986; Lalande, 1926; Starobinski, 1979). Arrivé summarized:

the symbol is not arbitrary but links naturally to what it signifies; (p. 19);

$\sim$ symbols change over time; (pp. 24-25);

$\sim$ a symbol has no identity of its own and can be separated from what it represents (pp. 26-27).

Characters and events in the myth narrative become meaningful through their representation of something other than themselves; in other words, they act as symbols. As symbolic representations, the characters are not situated temporally or spatially in terms of the world of the living audience but only within the context of the story world; that is, they are not identified with a particular location of place or historical time relative to the physical, mental, or emotional world of living humans. Storytellers are free to change and adapt the characters' symbolic signification according to new situations and circumstances as they arise in the lives of the hearers of the story, for whose benefit the 
story is told. The mutable nature and symbolic representation of characters and story plot allows for transport and transfer of the myth across geographic and cultural boundaries and accounts for the commonality of stories found amongst many different tribes, along with their application to a variety of circumstances. The characters and events evidenced in the three versions of Iktomi reflect this flexibility of symbolic representation and conform to the notion of symbol as Arrivé concluded.

\section{Myth as performance/verbal art.}

The Dakota culture lacked a system of writing; consequentially, storytelling was essentially a performed art. The storyteller carried the oral histories and mythologies of the people, committed them to memory, and transmitted them in the form of performed reenactments before live audiences. In recounting a tale, the storyteller would assume different voices for different characters and demonstrate their actions and behaviors to bring the story to life. The narrative competence of the storyteller was predicated on his or her ability to engage and elicit a response from the audience. By so doing, the storyteller facilitated the restructuring of social roles, imparted counsel, and educated his or her listeners about cultural values and practices (Wiget, 1987, pp. 315-317).

\section{Story structure, form, and function.}

Original story vs. version.

This section addresses the concern of validity regarding versions of a Native story rendered in a non-Native language such as English. Because a narrative paradigm presupposes meaning derived from a convergence of "history, culture, and character" (Tedlock, 1983, p. 90), it follows that a story taken out of its cultural context loses some 
of its efficacy, as its supposed original structure has been modified and rearranged through translation and reconstruction.

Tedlock (1983) observed that many Native American stories were documented from English narration rather than the native language. He felt that stories lost their true native qualities when related in a non-Native language (i.e., English) but then were somewhat reconstructed through the creative license of the collector, and the final results were subsequently regarded as versions of the original story (p. 32). This definition of version suggests that the three texts used here are no more than attenuated replicas of an original Iktomi story. Such a claim implies they lack something of their true nature because they were rendered in English, even though they originate from the Dakota culture. I refer to the texts as "versions" to indicate that they are all the same story, though variously rendered. In my analysis of the texts, I attempted to discover information in the data that could provide insight about the culture despite the fact that they were recounted in English (and one version was presented in English alone) and to show whether their value according to the definition of narrative myth has been seriously compromised.

The versions of Iktomi used here exhibit three very different literary styles and levels of embellishment, though they share a common source of origin. Zitkala S̆a (1901/1985) wrote her version in English and essentially made it her own creation, since she wrote from distant memory and in her own style. E. C. Deloria (1932) recorded her version from more recent memory in her best attempt to capture verbatim the narrator's oral rendition given in the Dakota language. She wrote her text in Dakota as she 
remembered it from the telling, then translated it into English and edited it somewhat to facilitate better clarity and comprehension for readers who did not speak Dakota. Like Zitkala S̆a, Deloria relied on her memory since the time of writing was somewhat removed from the actual telling by a short period of time. Jahner and Keller reported that they rendered the English translation of Walker's version from his previously untranslated notes written in Dakota; Walker occasionally amended texts parenthetically with his own comments or glosses yet left the original text otherwise undisturbed (Walker et al., 1983, p. xxv).

Story Sources: Three versions of the tale.

The distinct styles of the three versions of Iktomi are further clarified as follows. As noted in the introduction, our knowledge about Zitkala S̆a’s (1901/1985) version comes from her collection of Old Indian Legends. Included in the foreword of the volume is a brief biographical sketch of Zitkala S̆a, written by Picotte, who was, herself, a Lakota and native speaker of the Lakota language. Picotte recognized Zitkala's stories rendered in English as the same stories she remembered hearing recounted in the Lakota language (Picotte, 1985, p. xi). Zitkala S̆a used an abundance of descriptive detail to color the scene, characters, and action throughout the story and to personally involve her English-speaking readers. She strove to make the story real for her readers from the Indian point of view (Fisher, D., 1979, p. 50). In this regard, however, she transgressed traditional practice by inserting biased emotional content, as will be described in Chapter 4. Deloria was less prolific with flowery detail though included sufficient information to move the action and events along fluidly. She chose careful words in her translation that 
made sense to the English speaker although she also provided a literal gloss along with a free translation to aid comprehension. Jahner and Keller's translation of Walker's version revealed a script starkly devoid of detail, so that, for a casual reader, the story presents a dry and uninteresting text with occasional gaps in logic and connecting information.

Written vs. spoken record.

The difficulty of working with written versions of texts arising out of oral cultures engenders concern among scholars with regard to maintaining the integrity of their texts. In many cases, early collections were obtained through inconsistent methods or by untrained ethnographers, in part due to the absence of a systematic process that had yet to be developed. The rapid rate of disappearance of Indian cultures and speakers of their languages further necessitated hurried collection, leaving little time to attend painstakingly to details ordinarily considered vital to the documentation process.

Yet, conflict arose between the prevailing ethnographic methods and later ideologies which viewed narrative as performed art. In accordance with maintaining linguistic fidelity to the text, Boas stressed the importance of working with original transcripts, however both Hymes and Tedlock criticized his reliance on written transcripts and translations, claiming he ignored other dimensions of oral literature characteristic of performance art. They argued that stylistics and features of actual performance in situ must be considered as well as written documentation which, alone, they regarded as insufficient to ascertain a text's significance. Hymes and Tedlock insisted that without vocal representation, the text could not be adequately evaluated. 
Certainly, vocal characterizations do clarify uncertainties in printed text; however, since audio files of the original narratives of Iktomi are not available, this feature cannot be verified in this study. Boas astutely acknowledged "the form of modern prose is largely determined by the fact that it is read, not spoken, while the primitive [MK: Native] prose is based on the art of oral delivery" (as cited in Tedlock, 1971, p. 125, cf. footnote 52). On the one hand, Tedlock exposed the flawed logic of comparing Native and modern (non-Native) prose; on the other hand, Boas sought to bridge the transition from an oral culture to the written one.

Undoubtedly, subtle and not-so-subtle nuances in orally related texts have been lost over time resulting from the shortcomings of the transcription method of documenting them. Original texts unquestionably offer great wealth of information in many regards; nevertheless, while neither the original transcripts nor audio recordings were available for the purpose of this study, I demonstrate that they are not necessary for the story in its modified form to fulfill its function.

Many scholars, especially language and culture preservationists, believe the essence of story analysis is to revitalize what we can of its original function. Jahner, who edited a number of Walker's manuscripts, alleged, however, "all our effort is not to try to explain how life was long ago. It is to try to take what we find from way back then and restore some youthful vitality to its sounds and meanings" (Walker et al., 1983, p. 177) and, I would add, to make it meaningful for today. To grasp the significance of her statement, suppose a non-native speaker of English from an underdeveloped nation happened across a poem by the twentieth-century American poet E. E. Cummings. As a 
sample of English or even of American culture, it would make little or no sense to the reader unfamiliar with Cumming's style. Yet, one versed in the avant-garde would appreciate intended or personal significance from pondering the choice, orientation, and rhythmic layout of the written words in the poem. Paraphrased for the non-native English speaker, the poem can still be appreciated for its underlying message, including its symbolism and references to natural phenomena, even though the original elegance is not preserved. Jahner (1983) professed that knowing "the exact words of the narrator and...the performance conventions of the culture" were enough to comprehend and appreciate Native narrative (p. 178). Unfortunately, this study was limited to the words that have been preserved by ethnographers and translators who were nevertheless close to the original narrators and the culture of their origin. Hence, the best I had to work with was the documentation they left us.

\section{Cognitive approaches to narrative organization.}

\section{Frameworks of organization.}

Scholars have devised various frameworks to describe the active progression of events found in mythical stories across different cultures. For example, Labov's (1972) model of oral narrative posited a five-part structure, including the elements of (1) orientation, (2) complicating action, (3) resolution, (4) coda (a closing), and (5) evaluation (as cited in Georgakopoulou, 1997, pp. 9-10). Georgakopoulou used a similar model for her study of Modern Greek storytelling while Bierhorst (1975) adapted a progression model consistent with the narrative framework used by both, which conceptualized myth in terms of naturally occurring cyclic rhythms, to analyze the Aztec 
myth of Quetzalcoatl. Bierhorst concluded that the story progressed through the phases of opening incident, ritual combat, flight and death, underworld journey, and resurrection (pp. 405-406). Alternatively, the progression of stages may be conceptualized in terms of background/orientation, situation/incident, encounter/crisis, result and consequence, and eventual return back to the beginning.

Bierhorst interpreted the stages as "a progression through youth, maturity, demise, limbo, and resurrection" and further correlated them with orderly movement through the cardinal directions as well as with certain topographical features of the land (pp. 406407). Bierhorst's model thus accentuates multiple layers of meaning and seems more attuned to the Native cultural conceptualization of cycles and storytelling's purpose to transmit relevant cultural information. The pattern of cycles in Iktomi adheres to a similar cyclic pattern of progression, which illuminates its cultural relevance.

\section{Plot summary: Organization/structure.}

The elements of Iktomi closely conform to the narrative frameworks posited above. To show how the sequences of events in Iktomi fit into the frameworks, I charted them schematically with reference to two of the narrative models, along with alternate descriptions of the recurring cycles, as presented in Appendix A. The story plot progresses according to the frameworks' cycles which are indicated as follows by superscript numbers in parentheses corresponding to the phase cycle in the narrative models laid out vertically in the appendix:

${ }^{(1)}$ The trickster/spider Iktomi travels along aimlessly until ${ }^{(2)}$ he happens upon a group of ducks, whereupon he devises a plot to kill some to eat. ${ }^{(3)} \mathrm{He}$ deceives them 
through trickery, playing on their social values of trust and hospitality, and ${ }^{(4)}$ manages to kill a few. He prepares the ducks for roasting and ${ }^{(5)}$ sits back to wait while they cook.

${ }^{(1)}$ Presently, he becomes aware of a persistent annoyance in the environment near him and ${ }^{(2)}$ he sets out to subdue it. In a futile attempt to exert his control and influence he becomes caught in a tree from where ${ }^{(3)}$ he espies a potential adversary in the form of a wolf passing through a long way off in the distance. ${ }^{(4)}$ Anxious that the wolf will find his cache of roasts, Iktomi foolishly precipitates the wolf's discovery of it, ${ }^{(5)}$ drawing to himself the very thing Iktomi fears.

${ }^{(1)}$ Iktomi watches the wolf approach, feeling powerless to do anything about it.

${ }^{(2)}$ The wolf eats all of the roasting meat while Iktomi watches helplessly from his perch.

${ }^{(3)}$ The wolf satisfies himself that he has eaten all the roasts and departs, ${ }^{(4)}$ and then Iktomi is freed from the tree. Disgusted, disappointed, and still hungry, ${ }^{(5)} \mathrm{Iktomi}$ goes on his way.

Zitkala S̆a's story stops here though Deloria's and Walker's versions continue:

${ }^{(1)}$ Iktomi goes on to ${ }^{(2)}$ a second encounter with the wolf where, this time ${ }^{(3)}$ Iktomi has the upper hand and wreaks revenge on the wolf. ${ }^{(4)} \mathrm{But}$ the result is the same as in Zitkala S̆a's version and ${ }^{(5)}$ Iktomi moves on without having sated his hunger, again wandering aimlessly to his next adventure.

Here again we see a cyclical process in the story as it winds through the stages of the framework, moving through background/orientation, situation/incident, encounter/crisis, result and consequence, and return to the starting point. 
IKTOMI: A CHARACTER TRAITS ANALYSIS

\section{Structural continuity, memory, and recall.}

Further support for the structural continuity among the different versions used in this study that I have just delineated may be found in Mandler, Scribner, Cole, and DeForest (1980); they found that recall and comprehension increased when stories included and sequenced certain features, (as cited in Georgakopoulou, 1997, p. 5). Their finding lends weight to Zitkala S̆a's and Deloria's use of memory and recall to record their versions and their accuracy and conformity of details to each other's versions and to Walker's as well. Patterns of repetition and cyclical progression are discernible in each, and all follow the same logical sequence of encounters and events observable through their varying degrees of description. However, all three narrators exercised their freedom and discretion as individual storytellers to adapt their tale to their particular circumstances. Zitkala S̆a added an introductory segment at the beginning of her version, which is absent in the other two, and where her version ends, Deloria's and Walker's versions continue on with a renewed encounter between Iktomi and the Wolf.

Stylistic form: Performance art and the poetic style debate.

The study of Native stories has evolved through phases of interpretation and analysis, which I outline here in an attempt to background my approach to analysis of Iktomi.

Early documenters such as George Bushotter, James Dorsey, Stephen Riggs, and Gideon and Samuel Pond, among many others first made their invaluable contributions in collecting and recording cultural material relating to stories and language that has enabled subsequent scholarship and study of the cultures native to this continent. Initial analyses 
of narrative text data were primarily concerned with the linguistic aspects of Native languages and translation into English for use by religious missionaries as well as western researchers interested in understanding the cultures indigenous to the land. Later studies have attempted to reconstruct a humanistic sense of the people whose stories were documented and preserved (Walker, Jahner, \& SHSC, 1983, pp. 37-40).

In that context, the Iktomi story passed orally among storytellers at a time when no audio or visual documentation tools were available. To record the storytelling event, documentation involved writing, transcribing, and sometimes translating spoken texts, and it was not until the late 1960s that Western folklorists began to regard oral literature as a form of "performance" art. Within a few years, D. Hymes (1971) and Tedlock (1971), as mentioned above, advanced a shift in analytical focus from the documented text to the communication event itself, propelled by respective publications in the same volume of the Journal of American Folklore. Hymes and Tedlock had been equally critical of the paralinguistically deficient transcriptions of essentially dictated texts, the structuralism of Levi-Strauss and later adherents of his approach, as well as Boas's scientific method of reconstructing orally related texts. However, a hotly contested debate developed into an ideological split between the two as they pursued alternative approaches in their study of the nature of cultural narrative.

Concerned with the poetic style of narrative, both Hymes and Tedlock used a verse format for restructuring their texts for analysis to more accurately reflect traditional performance. Whereas D. Hymes (1975) arranged texts into segments - acts, scenes, stanzas, verses, and lines - based on word counts and repetition of numerical patterns, 
Tedlock arranged texts according to their content to draw out the poetic qualities present in the verse. Hymes attempted to identify a poetic fingerprint correlating to a specific culture and demonstrate that the rhythms in myth follow a numerical pattern attuned to the numbers considered sacred by the tribe (p. 369; cf. also Bright, 1987; Krupat, 1993). Tedlock (1977) acknowledged that "a metrical framework...may facilitate memorization" which storytellers rely on (p. 507; cf. Norrick, 2000) though he also pointed out that the notion of metered verse actually emerged from Latin during the Middle Ages and became a distinct literary form in Europe during the eighteenth century (pp. 512-513); hence, it most likely was not a feature of Native American poetics.

Through working with the Zuni, Tedlock (1983) noted that texts are not fixed but show variation according to a variety of factors and circumstances; they occur in multiple versions with the actual text influenced by the particular occasion, place, and time of the telling, as well as on who was in attendance, including the, teller, interpreter, and audience (p. 236). He also argued specifically that word counts do not accurately reflect the psychological or social impact of poetic style since they will commonly vary with voice inflection or omission of repeated components of the narrative. Further, they cannot account for intended meanings that are only implied or indirectly expressed (Tedlock, 1971, p. 132). Hymes eventually partially conceded that the relationships between the lines of text, which might be only a word or phrase, carry greater importance and that meter is not involved in narratives; (1981, p. 63). Hymes also back-pedaled on his original position, admitting that "the narrator always has options within the principles of patterning" and they are not restricted to only one type (p. 51). Yet, wherever Hymes 
detected variations in the patterns, he contrived a variety of ways to explain them away or simply ignored them, dismissing them as preferences rather than requirements of the text in cultural context (p. 52).

Tedlock developed a notational method to graphically represent spoken features in transcription in response to the deficiencies he found in Hymes' approach and in the traditional use of plain script, which cannot capture voice intonation and other paralinguistic features of performed text (as cited in Roemer, 1983, p. 46). Using audio recordings taken from Zuni storytellers, Tedlock, in effect, attempted to reconstitute the text in the transcript to an oral form. In his view, texts can only be understood when they are experienced aurally $(1977$, p. 516). Naturally, this is problematic for studying texts in languages whose fluent native speakers have mostly died out and for which no recordings are available, and Tedlock's approach is not possible with the three versions used in this study, which are available only in written format. Without audio transcripts, story progression and meaning must rely on its grammar and informational content.

It would be convenient to find that numerically organized structure exists inherently in cultural stories or that it naturally occurs in the patterns of individual thinking and regularly indexes culture, particularly with specific focus on sacred numbers. Yet, it seems presumptuous and unrealistic to expect that cultural myths would be deliberately patterned after number sequences and maintained in casual transmission from generation to generation and from storyteller to storyteller.

The three different versions of Iktomi rendered in English, either originally or from Dakota transcripts, make the possibility of rhythmic uniformity unfeasible since 
they feature so many variations in length and descriptive detail. To begin with, Zitkala

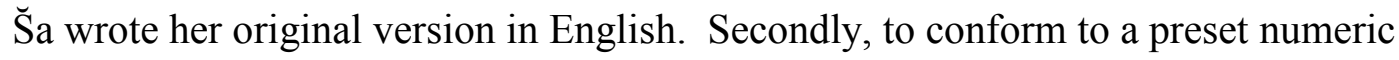
pattern, she would likely have styled her Indian legends after cadent nursery rhymes rather than fairy tale prose; Deloria would not have simply numbered her lines consecutively but would have also at least made paragraph divisions; and Walker's storyteller might not have been so stark with his verbiage. Of course, with Deloria and Walker the versions have been edited to some extent through the process of translating and committing them to written format.

Tedlock (1977) further argued that by attempting to poeticize the stories and transmute them into a formalized form of literary art in Hymes' manner, the whole point of the story becomes obscured because the point of the story is to transmit important cultural information that may be critical to the survival and well-being of the tribe in everyday life (p. 512). For as Levi-Strauss put it "substance does not lie in its style, its original music, or its syntax, but in the story which it tells" (as cited in Tedlock, 1971, pp. 120-121; Ballinger, 2004, p. 10). To a people for whom mythical narrative played so important a role in maintaining social good health and well-being, it seems likely that content would have taken precedence over presentation or that the two supported each other seamlessly.

Kroeber (1998) referred to myths as "imagined reenactments" whereby the storyteller interacts with his audience and makes the content subjectively meaningful for them. He observed that even apparently "trivial variations... may signal a major revision in the total meaning of a familiar story" (p. 70). Kroeber intimates here that, not only can 
the rhythm fluctuate, the teller can also revise the message! The flexibility to alter the story to any extent distorts the rhythmic flow that Hymes insists on. The power of the storyteller to manipulate texts suggests that, while a general pattern of theme may adhere to a significant number schema, the line-by-line rhythmic pattern cannot consistently be maintained, at least for this classification of stories. Kroeber also thoughtfully noted, "metrical regularities, elaborate metaphors and conceits, even detailed descriptions would interfere with the audience's participation in the verbal performance" (p. 147), the significance of which I elaborate on later in the section labeled "Essential components of myth narrative."

So, it would seem, the problem persists of how to approach documented text without imposing a preconceived concept of its construct as viewed through a Western cultural lens or forgetting that the Indian people oriented toward a different worldview.

While I concur that Native American storytelling constitutes a form of performance art and demands attention to stylistics separate from the grammar and linguistic form of the text itself, I chose not to attempt either Hymes' or Tedlock's approach in my analysis of Iktomi. Hymes' analyses proved partially successful, however, results were inconsistent with a proliferation of exceptions to his structure and the patterns that "emerged" at times appeared to be forced. (For detailed discussion and studies of recurring rhythmic patterns in myth, cf. D. Hymes, 1977, 1994; Hymes, 1981; Sherzer and Woodbury, 1987; Tedlock, 1983). Tedlock's method also never took a firm hold among scholars and researchers. Although his representation of paralinguistic features did capture a more stylistic feel for the text, it proved very cumbersome and 
difficult to manage. It can be argued further that his results reflected the individual style of particular narrators but cannot be generalized as a cultural model representative of all Zuni storytellers, let alone storytellers from other cultures. I circumvented these stylistic problems in my study by approaching the data from the standpoint of character traits analysis whereby, I was able to develop a systematic method that adapted to variable written texts without sacrificing the integrity of their cultural purpose.

\section{Story comparison across cultures.}

The protracted debate between Tedlock and Hymes also failed to take into account the fact that they analyzed the texts of two different cultures, respectively, Zuni and Chinookan; therefore, their arguments may ultimately hold only for their respective cultures of observation. Instead, it might be more useful to compare normal speech patterns with those in narrative as they apply to different contexts and situations within the same culture for patterns that align or diverge from one context to another. Tedlock (1983) noticed that stories would indeed be told on different occasions in different cultures. For example, Quiché Maya speakers would stop mid-conversation to interject a story that spontaneously came to mind whereas the Zuni planned their stories, telling them only at certain times and in certain prearranged settings (p. 515). It follows that different circumstances precipitated stylistic variations in the stories that were told. Jahner was more specific; she asserted that to adequately ascertain the stylistic qualities of a text it must be compared "with other versions of the same plot" (Walker et al., 1983p. ix). While Jahner's dictate makes sense, she did not follow her own recommendation but instead presented only a few examples of different stories from 
selected storytellers included in the volume, none of which overlapped. She left the task to future research, recommending sources for comparison, from which the two rival versions of Iktomi included in this study have been selected (pp. 407-408). However, as previously indicated, a detailed stylistic review is not the focus of this study.

Krupat recognized the trap that Hymes and Tedlock set for themselves by insisting on a "right" or "better" way to proceed with analyzing texts and accurately named it as a competition for authority (Krupat, 1987, p. 122; see also Mattina, 1987, pp. 130-137). He urged others to accept that there could be no single authoritative method for analyzing texts, particularly a method which held the prerogative of deciding what constituted authentic "Indian literature" (p. 122).

At greatest disadvantage in the search for texts to compare and analyze are those who wish to preserve and revitalize language that is no longer spoken or whose last truly fluent native speakers have all passed on, since the opportunity to obtain, test, and compare phonological information has dissolved with their passing. Also disadvantaged are those concerned with cultural continuity among stories of peoples who have been displaced from their native homelands and the identifying landmarks which are represented, albeit metaphorically, in the stories.

I do not intend to debate which approach is the correct or even the best one, and I present them only to demonstrate the contrast between my approach and earlier methods. In parallel with Jahner's suggestion to work with the same plot, I examined one story told in three different ways by three different storytellers involving starkly contrasting levels of detail to show that one, each of the renditions is a version of the same story and two, 
how the underlying message may be discernible through the characterizations, symbolism, character self-reference, and progression of events. Although interesting and informative, particular attention to the contour of the narrative was not a requirement for acquiring cultural insight from the Iktomi story, except that it reinforced what could otherwise be gleaned from the bare text.

\section{Aspects of myth.}

Authorship and ownership.

Original authorship of Native myths is not important to tribal people for the reason that stories are considered to belong to everyone and are passed along freely. Consequently, neither Zitkala S̆a nor Deloria made a point of recording the names of the narrators who contributed to their versions. Storyteller author Leslie Marmon Silko asserted that we will not find a "single distinctive and authoritative or personal voice" in Native stories because every telling of a familiar story represents a composite of all other tellings (as cited in Krupat, 1989, pp. 60-61; cf. also Ballenger, 1997; Vizenor, 1990). Silko added "oral tradition depends on each person listening and remembering a portion" (as cited in Krupat, 1989, p. 61; also cf. Huntsman, 1983). As to the Dakota ohuykakay, which were usually told in the evenings at the end of the day's activities, anyone qualified to tell a story although the privilege usually fell to the elders and more experienced members of the family or community (Picotte, 1985, pp.xiv). Kimball and Ghezzi recognized the same creative flexibility with the Koasati and Ojibwe cultures, respectively (Krupat, 1993). 
Many scholars have stubbornly preoccupied themselves with identifying the originators of specific versions of tales, ostensibly to compare variations and similarities among texts, styles, and dialects and to reconstruct lost culture. However, the focused attention directed toward specific individuals more aptly reflects the Western value of individual accomplishment than Native concerns. Identification of the specific narrator would be useful to the extent of socially and politically orienting the ethnographers' motivations for recording their stories and for detecting and deciphering bias. However, that Deloria’s and Zitkala Ša’s narrators remain unidentified limits discernment of personal association or influence by a narrator with a particular version. Any detected bias or influence must be attributed to the ethnographers themselves operating as filters of the text that reaches us.

As with other aspects of Dakota culture, the gifts (i.e., talents, abilities) and information that are handed down generationally or granted through communion with the spiritual powers are meant to be shared with all the people to help the tribe as a whole. In Native contexts, the storyteller does not receive his or her gift for personal gain but must accept the responsibility to transmit knowledge and information as communal property. Through the storyteller's gift, the stories become a part of the cultural heritage owned and shared by all, and each individual retains editing privileges.

\section{Story variation and adaptation.}

Stories circulated widely and freely with frequent variation. Narrators assumed a level of shared cultural knowledge with the audience and elaborated with more or less detail as needed by their listeners, as Tedlock (1971) noted among his Zuni storytellers. 
Dakota storytellers also employed ellipsis liberally, leaving the audience to contemplate and implicitly infer their own conclusions. Omitting details and certain connecting information enabled listeners to develop their own judgment and reasoning ability, weigh proposed actions against their consequences, and strike a balance between their own personal interest and the welfare of the group. The storyteller in effect acted as facilitator, educator, and counselor guiding members of society along the way in "life as it ought to be lived" (Benjamin, 1969, as cited in Fisher, W. R., 1987, p. 73, cf. endnote 70).

\section{Character nature and role in culture.}

In the genre of the Dakota ohunkakay, mythical events take place in the distant past at a time when people and animals were one and the same and all possessed supernatural powers (Bierwert \& Indiana University, 1996, pp. 63). Even plants, minerals, and elementals all held powers of intelligence and reasoning, a concept still considered true in the culture today (Smith \& Allen, as cited in Ballenger, 1997, p. 798). The characters in the story of Iktomi-Iktomi, Ducks, Skiská, ${ }^{2}$ Wolf/(ves), Tree(s), Wind, Fire $^{3}$ - all intimate various levels of consciousness and intentionally act on each other; although by Western standards, none are human, and only three of the seven are animate. Each character exhibits human traits, behaviors, and emotional reactions in order that the listener might recognize his or her own reflection in its portrayal and thereby contemplate his or her own social and moral standing among other members of the tribe. Using

\footnotetext{
${ }^{2}$ Skiská is the Dakota word for "wood duck". In the Iktomi story, "Skiská" is given as the character's proper name.

${ }^{3}$ Wind and Fire will be shown to be noncharacters; however, they are included here since I initially considered them as potential characters. I explain my process of elimination in Chapter 3.
} 
nonhuman characters to represent human expressions places distance between the listener and aspects of personality and behavior that he or she might recognize and identify within himself or herself while creating a non-threatening venue for the individual to address those qualities deemed socially inappropriate.

Other animals in myths serve more as a plot device than as conscious characters. For example, game animals such as deer typically represent sources of food (Bierwert \& Indiana University, 1996, p. 65). The Ducks in Iktomi also play the role of food yet they initially act as sentient beings which actively and consciously engage with Iktomi and provide a vehicle for communication to help us gain access into the inner workings of Iktomi's mind. Once the relationship has been established and Iktomi's motivations are revealed, the Ducks relinquish their individual identities and personalities and transmute into impersonal, lifeless meat. It is interesting to note that Iktomi always regards the Ducks as food although he engages with them as conscious entities akin to himself, albeit as his inferiors.

\section{The trickster in Native American culture.}

The title character Iktomi is a trickster figure in Dakota mythology. In the context of Native cosmology, Horne's (1999) detailed description of the Native American trickster and its role in culture places it as a figure which existed prior to the creation of human beings and which represented the first prehuman people to inhabit the earth.

Then, when humans were created, the tricksters transformed into animals but retained the ability to shapeshift into other forms. Tricksters are characteristically situated outside of space and time, and although they loom larger than life, people within the culture regard 
them as real entities. Tricksters exhibit a vast array of desirable and undesirable traits, which usually represent extreme violations of cultural norms and social acceptability. No one and nothing ever vanquishes tricksters; they may die, become disfigured or mutilate themselves yet they always resurrect and restore themselves to their original state. They often play the fool though while deceiving others, they themselves become deceived. Through their travels and exploits, the tricksters eventually reach a crossroads or borderland, where they must pause before making a decision or choice. Their acts and movement serve to restore balance in the world by bringing into awareness how the people's thoughts and actions caused it to wobble off track. They reflect our own images of who and what we are in addition to the possibilities of what we might become. Tricksters do not abide by social rules and protocols; rather, they flagrantly snub them and unashamedly cross the boundaries of right conduct. Their mishaps and foolishness create awareness in others of likely consequences of wrong actions and poor decisions (cf. pp. 127-130).

Let me point out that in all three versions the trickster Iktomi is always male, a point to which I will return in Chapters 4 and 5.

Horne (1999) claimed that those unfamiliar with Indian culture and traditions would not grasp the cultural significance built into the trickster stories. Consequently, the stories provided a culturally relevant outlet Indian people could use to vent and discuss discontent with the dominant society during certain periods without its discovery of hidden undertones (p. 131). Horne's allegation may be one reason to explain why the 
stories have eluded scholars; quite possibly, cultural information may have been intentionally kept hidden from outsiders in order to protect Native interests.

The trickster in Plains Indian lore is represented by Iktomi, a character which takes the form of a spider (Ballinger, 1989, p. 39) and is analogous to tricksters of other Native American cultures, for example, Raven or Blue Jay in the American Pacific Northwest, Hare in the Southeast, and Coyote of the western United States and Mexico, among others (Bright, 1987, p.341). Trickster figures are modeled somewhat after their animal namesakes; however, their characteristics range far more broadly and encompass traits and qualities of humans, elementals, and supernatural forces. For instance, Mink in the Coastal Salish Lushootseed mythology parallels the characterization of Iktomi in the Dakota stories ${ }^{4}$; neither is located in time nor space (DeMallie, 1993, p. 530) and like Mink, Iktomi travels aimlessly until he unwittingly happens upon an opportunity to satisfy a particular desire - in this case, to obtain food. Motivated by self-gratification, both craft a deceitful plot to achieve their aim, succeed, then, spend an amount of time preparing to savor it. Somehow each is thwarted in his endeavor and finally, they move on in an unspecified direction without further apparent purpose.

Like other cultural tricksters, the identities of Iktomi and Mink are readily recognizable even outside of any particular story. "When a narrator mentions Mink's [i.e., Iktomi's] name, the audience already knows all about him" and knows to expect something (Bierwert, 1996, p. 64). Recognition by name enables the audience to immediately grasp culturally relevant details without the narrator's need to spell them out

\footnotetext{
${ }^{4}$ A Lushootseed story similar to Iktomi is the Changer Story about Mink told by Martha Lamont (cf. Hess, 1996).
} 
in the story and to anticipate an aspect of thinking or behavior to watch out for. This character recognition accounts for at least some of the brevity of descriptive detail present in different versions, and the amount of description needed depends on the audience's cultural savvy.

According to post-modernist Vizenor (1989), social scientists have tended to partition off the trickster and distance him from other elements of the narrative. However, the trickster does not operate in a vacuum but is integrally and intimately involved with every other facet of the story and its participants. Vizenor concurred with renowned literary theorist Bakhtin's claim that "the interlocutors in the trickster narratives are the author, narrator, characters, and audience" (Vizenor, 1989, p. 191). In other words, the trickster embodies the composite of everything in the environment and can identify not only with every participant involved in the story but also in the telling of it. Once invoked, the trickster does not stand apart from the action; he manifests himself in every aspect of every individual, the circumstances, and the reason for the story. This changeable nature of the trickster lends itself to identification with the story's participants.

Vizenor elaborated on the trickster's mirroring role: "Trickster is a collective shadow figure, an epitome of all the inferior traits of character in individuals" (p. 205). The trickster usually, although not always, illuminates those qualities about ourselves that we don't want to acknowledge, those we consider in a negative light. Ultimately though, his purpose is to bring everything back into balance, including the excess of positive attributes. Both Velie and Vizenor viewed the trickster as a "culturally centered, 
communally created, highly complex, comic figure who cannot be isolated from or understood outside of the context of his discourse" (Velie, 1989, p. 131; cf. Vizenor, 1989). These opinions coincide with those of noted trickster scholar Radin, who, in his earlier work, identified the iconic trickster as indicative of human nature: "What happens to him happens to us" (as cited in Roemer, 1983, p. 40, footnote 7). The important notion of audience identification with the trickster and the story's participants will figure prominently in the results discussion in Chapters 4 and 5.

Story in context.

Scholars repeatedly return to the issue of context and generally agree that the story's significance can only be ascertained against the backdrop of the culture. In accordance with this stance, Roemer (1983) recommended that the reader (listener) "should have some relevant knowledge of the particular tribe's culture, history, and environment" (p. 42). Relative to the three versions of Iktomi, this means the figure of Iktomi can be understood only in the context of Dakota culture. A cultural outsider still may possibly find an entertaining story or perhaps discern a message that can be linked to a Western experience; however, if Roemer is correct, outsider understanding can only be modestly superficial unless informed by important "local" knowledge. Further, according to Roemer, "the reader needs to know relevant information about the language, genre, and style of the story" in order to decipher the information available in it (p. 44). Again he claims proprietary comprehension exclusive to cultural insiders. Roemer's stance negates the ability of a trained analyst outside the culture to approach a cultural 
text with any competence and further implies that any given story is relevant only to its culture of origin.

Taking a different position, DeMallie (1993) elaborately discussed comparison and contradiction between historical accounts narrated from different cultural perspectives. He argued that different accounts arise "because they are composed for different purposes, and are based on different cultural premises" (p. 532). Taking DeMallie's perspective into account, my study is an attempt to uncover encoded cultural information embedded within these versions of Iktomi without the benefit of assistance from a native speaker who lived at the time they were recorded and to glean value and insight that may be relevant to a contemporary audience, including a non-Native one. I contend that if the stories truly meet the requirements of timelessness and adaptability to environmental conditions, they should continue to offer guidance and relevance, regardless of the audience's cultural heritage and language background. To argue otherwise places unnatural limitation on the notion of culture, which we know to be dynamic and ever-changing. As stated earlier, stories are a human universal, and surely we can all recognize the trickster at work in our own lives, as Vizenor, Velie, Bahktin, and Radin suggest.

Ultimately, I find both Roemer and DeMallie's positions to hold true with regard to Iktomi - the story does carry cross-cultural relevance, yet simultaneously, it encodes a very specific framework for a culturally specific Dakota ritual. The latter point is borne out in my interpretation of the analysis in Chapter 5 while the cross-cultural aspect of the story may be understood through 
the application of the three narrative voices and through the interaction between the four essential components of myth narrative, which I describe shortly.

\section{Story content relative to purpose.}

Conventionally, emotional content is not explicitly expressed in Dakota myths but is left open to individual experience and interpretation. While the characters' thoughts, actions, and consequences are delineated, their response to behavior and events is not dictated to the audience, freeing listeners to experience their own genuine emotional reactions (Gunn Allen, as cited in Ballenger, 1997, p. 795). Deloria and Walker adhered to this formula; however, Zitkala S̆a blatantly steered the reader toward an empathetic alignment with the Indian's predicament as evidenced through Iktomi's unfortunate exploits.

Dialogue between characters serves as the mechanism used to help the plot progress and movement occurs as characters encounter and interact with other elements in the environment. In this way, each encounter sets in motion a contrast between an action or decision and its consequence (Walker et al., 1983, p. 165). As the character engages with another character or confronts a dilemma, he is faced with a choice between right and wrong behavior. Each option offers both something the protagonist desires and a deleterious consequence. Through the humorous antics of the characters, the story illuminates the conundrum and informs the audience of what outcome may ensue from a certain chosen course of action. The skilled storyteller may use the story as a preventive measure to ward off an impending situation or, conversely, in hindsight, he may use the story to point out how a negative behavior or wrong choice has impacted the community. 
In either case, he reads his audience to ascertain the current need and chooses a story appropriately relevant to the circumstances.

\section{Theme.}

I set out in this study to discover whether the Iktomi story contains accessible information that can be used in personal daily life. In the Dakota worldview, this would naturally include a spiritual dimension. Bierhorst suggested that Indian mythology tends to be themed around "guilt, death, and the quest for food" (1975, p. 408; cf.

Quetzalcoatl). While he captured a considerable measure of the essence of ritual symbolism and archetypal significance of mythical events, it seems he also missed an important component of interpretation which is how to make it relevant for day-to-day living. Unfortunately, scholars have largely ignored or danced around the spiritual dimension of Indian tribal culture, which shares equal, if not greater, importance in the lives of the Indian people. It is not too difficult to cull the references to important symbolism from a tale; however, to fully comprehend the cultural, meaningful, and usable significance of the story, its underlying spiritual aspect must be considered as well within the context of everyday life. In order to access the interwoven themes, I had to look beyond the surface interpretation of the text to its symbolism which I found to be discernible through closer inspection of its characters.

\section{Three narrative voices.}

Momaday spoke of "three distinct narrative voices...the mythological, the historical, and the immediate" (as cited in Roemer, 1983, p. 51). Using an example from his own life, he showed how myth narrative transcends time and space to accommodate 
changing lifestyles. He took traditional and historical oral narratives he collected from the Kiowa and incorporated personal and family experience into them to arrive at an updated, more contemporary version more applicable to his family's current circumstances. And it seems these three voices have also been applied in the Dakota myths; in each of the versions of Iktomi, the storytellers have taken a mythological event representative of historical Dakota experience, related it to a more modern time, and applied it to the situation at hand and the circumstances of the attendant audience to address their immediate need or concern.

\section{Essential components of myth narrative.}

In accordance with Silver and Miller (1997), I identified four essential components that comprise myth narrative: (1) narrator; (2) audience; (3) context; and (4) situation or setting. Contrasting oral narratives with written versions of narratives, Silver and Miller found that written versions usually lacked "a sense of the storyteller, the audience, and the place and time of the telling," which they considered crucial to Native literary form (p. 122). Kroeber (1981) espoused the critical roles of narrator and audience and the interplay between them as a "practical social transaction" in which both engage interactively in an imagined reenactment of a practice within a sociocultural context (pp. 62-64). Kimball (1993) maintained that, due to their nature as an oral art form, narratives exist only in the mind of the narrator, whose responsibility is to give rise to their shape and form; whereas, the audience in attendance at any given place and time influences the ultimate product of the narrative (p. 4-5). Indeed, it is such observations 
that prompted Hymes' and Tedlock's critique of the Boasian tradition and their own arguments with one another.

Clearly, in order for the narrative to be meaningful and make sense, the events that take place in the narrative must be believable and possible in the setting in which they are placed.

With regard to situation as an element of storytelling, historically, tribal storytellers used traditional stories to help Indian peoples cope with changes in conditions and circumstances resulting from the stripping away of their old cultural ways. Storytellers adapted the stories to different places and times with each telling depending on the circumstances and the emotional needs of the listeners (Kroeber, 1981, pp. 276277). Although, by definition, Dakota myths originated from a time preceding human emergence, many have been regarded as applicable to more current historical times and seem to depict and describe events experienced by persons who lived through them. It is this feature that gives them their universality. Further adaptations have arisen based on gender of the narrator, multiplicity of purpose, interpretation, whether told for an Indian or non-Indian audience, and to accommodate constraints on time, as in the case of dictated narration (Kimball, 1993, pp. 4-5; Kroeber, 1981, pp. 145-153).

\section{Narrator.}

In traditional Native communities, the narrator or storyteller was a "carrier" of stories and a vital member of the tribe who fulfilled the very important function of preserving and transmitting the oral history of the people. As such, the storyteller was respected for his or her knowledge of tribal customs and values, tribal resources, and the 
topography of the land on which they lived and subsisted and over which they migrated. Storytellers modeled an exemplary code of behavior and speech and were socially sensitive and perceptive to subtle indications in the mood and temperament of the group. They were also familiar with aspects external to the community, since they frequently carried information from other villages or societies (cf. Krupat, 1989, p. 59; Tedlock, 1983).

Becoming a storyteller was taken seriously and typically involved lengthy guided training via a holistic process of memorizing stories and myths, accumulating knowledge of tribal lore, and developing the skills and abilities to read people and situations in order to apply story material to the appropriate context (Clements, 1996, pp. 130-131).

A modern day Oglala Lakota storyteller, Jerome Kills Small summed up his perception of what makes a storyteller: "retelling and redefining those histories of people, and [making them] current, re-explaining [them],... [and] to be a good filter for all the people who would listen" (Zimiga, 2010).

\section{Audience.}

As noted above, the story narrative involves both narrator and listeners and represents an interaction between them. Brice Heath indicated that "listeners play a necessary interpretive role for the story" and help to shape how it unfolds (1986, p. 90). Thus, the storyteller depends on the audience's response in order to proceed through the story (Krupat, 1989, p. 62; Wiget, 1987, p. 316). As co-creators of a version of the story, narrator and audience access the myth's timeless adaptability and together bring it to life in a meaningful way that is relevant to the immediate context. 
Tedlock (1983) also noticed that his storytellers adapted and censored their material depending on who was in attendance, whether the audience consisted of all males, males and females, adults or children or both, or someone with a recording device which would permit the story to be heard by a much broader and unknowable audience in the future (p. 292). He also found that censorship occurred according to the sensitivities of those present (though not based on topical explicitness) including potential future audiences. It took a very skilled storyteller to read such a varied audience and anticipate future participants not yet present. This facet of future potential reinforces the amount of skill and training that was required by an adept carrier and preserver of tradition and demonstrates the inherent need for flexibility and adaptability in the story.

\section{Context.}

Context describes the immediate circumstances of the audience or the antecedent circumstances that have given rise to the story; in other words, it comprises the reason the story is told. The context includes the psychological and emotional states and needs of all of the participants, inclusive of the audience, narrator, and those standing by potentially uninvolved. The context may be thought of as the story's topic or theme, involving a psychological or emotional component that one or more of the participants must wrestle and come to terms with. Using skills of observation and intuition, the narrator identifies an element of need that arises from those present, perhaps perceiving a conflict between family or community members or the internal struggle of an individual who may be having a hard time with emotional conflict or indecision. Then, drawing on 
his or her store of knowledge, wisdom, experience, and cache of stories, the narrator weaves a tailor-made tale to address the source of discomfort and alleviate distress.

\section{Situation.}

Another essential component of narrative is the situation or setting in which the telling occurs. In contrast with the context which alludes to the psychological impetus of the storytelling event, the situation or setting refers to the worldly surroundings, atmosphere, physical conditions, and people, animals, or objects that are present as the backdrop for the storytelling event. The elements of time and space within the story world are vacuous concepts; as a consequence, rather than referring to the time and locale in which the story takes place, narrative setting pertains to the immediate physical environment of the attendant audience which includes all the participants including the audience, narrator, bystanders, and the presence of recording devices. The situation describes the specific date, time of day, and physical location of the telling, as well as other elements that might directly or indirectly influence how the story will unfold. All of these variables will likely affect the narrator's decisions about material to include, mode of expression, and specific interaction with members of the audience and the immediate environment.

\section{Part 2: Documenter Ethnography}

As introduced at the beginning of the chapter, in this section, I discuss the background and orientations, methods, and motivations, of the three individuals who brought these different versions of the Iktomi story forward from their source in the Dakota culture. The personal experience and background of the documenters would have 
naturally colored their perspective; consequently, each would have filtered the narrative they heard through their own personal frame of reference. The information I present in the following sections will help elucidate that personal lens with respect to the three documenters and give the reader a sense of how and, perhaps, why the versions were rendered so differently. The influence of the documenters projected onto the renderings becomes important to the analysis in terms of the detail provided in the narration, the slant toward individual characterization of story characters, and the underlying purpose for preserving the story as rendered in the three different versions.

The three versions emerged through the ethnographic work of Zitkala S̆a, Ella Deloria, and James Walker, whose contemporary lives and activities traced similar routes and involved many mutual acquaintances. The two women descended from mixed-blood Ihanktunwan $\left(\right.$ Yankton $\left.^{5}\right)$ Dakota families and spent their early childhood immersed in their traditional Indian culture. Then, during adolescence, they received formal education at Indian boarding schools, and both subsequently earned college degrees and launched professional careers. Walker was a White man born and raised on a family farm in southern Illinois. He received his primary and secondary education from the local school, earned his medical degree from university, and eventually established a career as a medical doctor.

\section{Documenter background and orientation.}

\section{Ethnographers: Birth place, family life, and historical perspective.}

Biographical information about Zitkala $\breve{S}$ a may be found in introductory inclusions to two of her published collections: Agnes Picotte wrote an endearing tribute

\footnotetext{
${ }^{5}$ The Yanktons were prairie dwellers living east of the Missouri River in North and South Dakota.
} 
to Zitkala S̆a in the Foreword to Old Indian Legends (1901/1985) and Susan Rose

Dominguez wrote an enlightening introduction to American Indian Stories (1921/2003).

Additional information comes from Dexter Fisher's (1979) scholarly essay titled “Zitkala S̆a: The Evolution of a Writer."

Most of the biographical information on Deloria may be found in forewords and prefaces to her own works, written by relatives and editors of her collections.

Contributors include Agnes Picotte and Paul Pavitch, who edited a volume of English language versions of E. C. Deloria's 1932 collection of Dakota Texts; her nephew Vine Deloria, Jr., who introduced the 1998 publication of E. C. Deloria's Speaking of Indians, originally published in 1944; and her granddaughter Joyzelle Gingway Godfrey, who prefaced E. C. Deloria's The Dakota Way of Life, which was published posthumously in 2007.

Editors Raymond J. DeMallie and Elaine A. Jahner provide biographical information about Walker in their detailed sketch "His Life and Work" in Lakota Belief and Ritual (Walker et al., 1991, pp. 3-61), the second in a series of volumes containing an edited compilation of his manuscripts.

James R. Walker.

The eldest of ten children, Walker was born early in the year of 1849 near the tiny village of Richview, Illinois, which lay a mere 60 miles north of the crossing of the Cherokee Trail of Tears that traversed the southern tip of the state. At the early age of 14 , he ran away from home and enlisted in the military, served only a few months on the Union side, and was discharged on account of illness. He resumed his education and, in 
1873, went on to acquire a medical degree from Northwestern University near Chicago; he subsequently married, had a daughter, and began his 40-year-long medical practice (Walker et al., 1991, pp. 3-4).

Walker was still quietly practicing medicine in Illinois when gold was discovered in South Dakota's Black Hills, driving the governmental mandate to move Indians onto the reservations. In 1878, he took a post working with the Ojibwe Indians at the White Earth Agency at Leech Lake, Minnesota. White-Indian relations everywhere grew increasingly touchy, fearful, and untrusting, and both sides rubbed itchy trigger fingers. Trouble brought Walker's five-year service with the agency to an abrupt end in 1893 when, as the agitation around Sitting Bull's death and the debacle at Wounded Knee had barely cooled off, Walker inadvertently shot one of the Agency Indians while trying to confiscate whiskey. The altercation reignited Indian unrest, necessitating Walker's reassignment to the Colville Indian Reservation in Washington State, where he remained for three years. From there, he transferred to the US Indian School at Carlisle, Pennsylvania; though, after only five months at Carlisle, for reasons unknown, Walker transferred again, this time to the Pine Ridge Reservation, where he would serve as Agency physician for the remainder of his professional career, some eighteen years (Walker et al., 1991, pp. 4-6).

Wherever he went, Walker witnessed first-hand how the imposition of harsh conditions and treatment impacted the Native people. By the time he arrived at Pine Ridge, the government had cut off aid to the Indian missions, subjecting the Natives to severe shortages and hardship, while disease spread rampantly among the disconsolate 
populace. He found his niche, working to bring the infectious elements under control, which led to the development of his longstanding alliance with the traditional medicine men of the tribe and the ethnographical volumes that he would amass (Walker et al., 1991, pp. 7-12).

\section{Zitkala $\breve{S}$ a.}

Zitkala S̆a was born in 1876 amid the Centennial celebration of the United States' victorious stride to independence from Great Britain (Zitkala S̆a, 1901/1985, p. xii). Steeped in its patriotic spirit of jubilation, the unsympathetic US forcibly moved the Indians off their own lands into squalid conditions on reservations carved out of their once vast territory. In the same year, Sitting Bull retaliated in war against the White trespass into the Indians' sacred Black Hills while amassed tribal warriors outmaneuvered Custer in the Battle of the Little Big Horn in nearby Wyoming (Karolevitz, 1975, pp. 115-119; Robinson, 1966, pp. 102-103).

Zitkala S̆a was born on the Yankton Sioux Reservation in South Dakota as the ninth and last child of her full-blood Ihanktunwan mother and third child of her White father, named Felker, who abandoned his family shortly after his daughter's birth. Zitkala S̆a's mother had married twice prior, each time to White men; yet, through all her marriages, she persisted with her traditional Nakota lifestyle and language and raised her children accordingly (D. Fisher, 1979, p. 231; Zitkala S̆a, 1921/2003, p. viii).

At birth, Zitkala S̆a was named Gertrude, and she first went by her father's surname; she later dropped the name Felker and took the name of her mother's second husband, Simmons. Later, however, as a result of a dispute over her legitimacy with her 
brother's wife, Gertrude cast off his name as well and created an entire new identity for herself as Zitkala S̆a, meaning "red bird" in Lakota. As an adult, she reverted once more to her given name and, later, included the family name of her husband, Ray Bonnin, which she used as her professional identity; however, she continued to use "Zitkala S̆a" as a pen name for her early publications and collections of stories (D. Fisher, 1979, p. 231; Zitkala S̆a, 1901/1985, p. xi).

Zitkala S̆a had just reached the age of eight when missionaries from White's Manual Labor Institute in Wabash, Indiana persuaded her, against her mother's wishes, to come away with them from the instability of her home life. However, the young girl belatedly realized the dupe when she arrived at the school; strict teachers unmercifully attended the new arrivals with grim austerity, introducing them to a regimen of unmeasured cruelty and abuse. Although White's was Quaker-run and Quaker families were generally kind to Indian children sent to work in their homes, the boarding school adhered to the US government's harsh policy of assimilation forbidding the children to speak their native language and behave according to their native customs. Punishment for disobedience came swiftly and painfully and the Native children quickly learned to dissociate themselves from their cultural ways. After three years at White's, Zitkala S̆a returned home; however, she found her school experience had changed her attitudes and behavior so that she no longer felt she fit in with her Native people. At the same time, her childhood friends and relatives also newly regarded her with suspicion as an outsider, i.e., a person from outside the community and, therefore, someone not to trust (D. Fisher, 1979, p. 232; Zitkala S̆a, 1921/2003, pp. ix-xi). 
Zitkala $\breve{S} a$ went on to the Santee Normal Training School in Nebraska for three years, after which, she returned again to White's Institute to finish her secondary schooling. She continued to return periodically to her family's home on the reservation; however, she never quite lost her out-of-place sense that she belonged neither in the White world nor among her own Indian people (D. Fisher, 1979, p. 232; Zitkala S̆a, 1901/1985, pp. xii-xiii).

Over the years following her boarding school experiences, Zitkala S̆a became an accomplished writer, musician, and activist. She completed her college degree in 1897 at Earlham College (another Quaker institution) in Richmond, Indiana, while concurrently attending the Boston Conservatory School of Music. From there, she taught at Carlisle Indian School, published collections of stories and essays, subsequently married, gave birth to a son, and moved with her family to the Uintah and Ouray Reservation in Utah, where she began to develop in earnest her role as activist, spokesperson, and advocate for Indian rights, especially on behalf of Indian women and children. Under the name of Gertrude Bonnin, Zitkala S̆a established herself as an influential force in Washington DC and helped establish Native American societies, clubs, and publications to progress awareness and betterment for the Indian people (D. Fisher, 1979, pp. 232-236; Zitkala S̆a, 1921/2003, pp. xii-xvi).

\section{Ella C. Deloria.}

Ella Cara Deloria was born in 1889 , the fourth in a family of nine children, to mixed-blood Ihanktunwan parents on the Yankton Indian Reservation in the midst of severe hardship for the Indians (Deloria, 1998, pp. x-xi). Volatility, tension, and hostility 
toward Indians had reached new levels on the reservations, as the US government had cut rations to the agency Indians, carved up and appropriated treaty-protected reservation lands, and systematically harassed nearby Indian encampments without provocation. However, Deloria's family had earned a high degree of status in the Indian community: her grandfather had held positions as tribal leader and medicine man on the Reservation while her father served as ordained Episcopal minister. Consequently, she grew up under both strong Christian and traditional Native influence in a relatively stable family environment among friends and relatives who spoke their Native Dakota language (Deloria \& Godfrey, 2007, pp. vi-vii; Picotte \& Pavich, 1992, p. v).

A year after her birth, Deloria's family moved far north to the Standing Rock Reservation, still in South Dakota, where her father founded St. Elizabeth's Mission School in Wakpala. Within months, Sitting Bull was captured and killed less than 50 miles from her home, while his remaining followers fled to their deaths in the Wounded Knee Massacre on the Pine Ridge Reservation further south (Picotte \& Pavich, 1992, p. v).

Amid the growing turmoil, six-year-old Ella began her education at St. Elizabeth's and eventually transferred to All Saints Episcopal High School, a boarding school in Sioux Falls, South Dakota. Subsequently, she attended the University of Chicago, Oberlin College in Ohio, and finally, Teachers' College at Columbia University in New York, where she completed her bachelor's degree in Education in 1915. After graduation, Deloria taught briefly at All Saints Episcopal, then at Haskell Indian School in Lawrence, Kansas (Picotte \& Pavich, 1992, p. vi). 
While at Columbia, Deloria began her association with Franz Boas, working for him in the anthropology department, and he hired her again in 1927 to collect linguistic and ethnographic material from the Dakota Indians. Although Deloria never attained professional status in her capacity as linguistic scholar and ethnographer, she continued the work off and on throughout her life, alternated with taking care of her mother and siblings back on the reservation, and she published a number of works in professional journals and independent volumes. In particular, her 1932 published collection of stories in Dakota Texts presented a huge contribution to ethnographic anthropology and continues, still, as an important source work for study and research of the Dakota language and culture (Walker et al., 1983, pp. 36-37).

\section{Crossed paths.}

In an ironic twist to their lives, all three ethnographers moved away from their respective natural home environments to experience that of the others. The traditional Native women, versed in their cultural customs and fluent in their native language, became educated and assimilated into the White culture. Conversely, the White man, immersed and educated in White culture, went to live among the Indians on the reservation and learned their customs, traditions, ceremonies, stories, and native language. This reciprocity of experience converged over time into a common purpose and motivation among them, which drove their work of collecting the stories from the old tribal storytellers and recording them for future generations.

Walker, Deloria, and Zitkala S̆a traveled many of the same paths, shared similar experiences of culture and place, and inspired and motivated, or fell under the influence 
of a number of the same people during the course of their lives and work. For example, although separated by years and then by distance, Deloria and Zitkala S̆a both hailed from the Yankton Reservation and would have known or heard of the same people, some of whom were likely mutual relatives. Zitkala S̆a had known and corresponded regularly with another noted Native American writer, the Dakota Charles A. Eastman (Ohiyesa), having met him at the Carlisle Indian School and discovered shared sympathies; Walker retraced Eastman's footsteps at Pine Ridge, where Eastman had served as agency physician four years prior to Walker's assignment there. The tribal elders, with whom Walker had developed intimate relationship, had spent similar time with Eastman during his medical tenure on the reservation. Deloria had also met coincidentally with Eastman along in her quest to obtain Native materials and through her own efforts to create social awareness on behalf of their Indian people.

Walker's and Deloria's connections further intertwined; Walker had provided numerous cultural and language materials to Clark Wissler, Boas's assistant and successor as head of the anthropology department at the American Museum of Natural History. Besides Boas, with whom she worked and collaborated extensively, Deloria worked and studied with other anthropologists at Columbia, who would have been at least aware of Wissler, if not in direct contact with him.

Indian Agent James McLaughlin represented another link between Walker, Deloria, and Zitkala S̆a. McLaughlin had investigated the incident involving Walker at Leech Lake that had resulted in his removal from the agency; later, McLaughlin transferred to the Standing Rock Reservation, where Deloria would spend her childhood. 
McLaughlin's half-Yankton wife Marie shared Deloria’s and Zitkala Ša's traditional upbringing; Marie later collected and compiled her own accounts of Indian myths told to her by storytellers on the reservation, many, of which closely replicated those published by Zitkala S̆a and Deloria.

Finally, of the informants sought out by Deloria and Walker, George Sword figures prominently as a frequent and common source of information. As one of his tribe's most gifted and creative storytellers, respected elder, and medicine man, Sword provided a great deal of the material found in Walker's manuscripts (Markowitz, 1996, pp. 617-618); Sword would have involved himself in many matters concerning tribal welfare, including the dissemination of information through stories that might perpetuate the culture and traditions of the Dakota people. Through him and others of his status, tribal knowledge in the guise of stories would have passed naturally through the receptive discernment of Native collectors and writers like Deloria and Zitkala S̆a.

Although Walker, Deloria, and Zitkala S̆a would all have obtained intermittent access to many of the same storytellers, the differences found in their respective versions of the Iktomi tale supports the claim that their material for this particular story did not emanate from the same source. Still, their movement, activities, and motivations indicate a cohesiveness of shared purpose and intention and highlight their circulation among a relatively small cluster of altruistic reformers, who shared the goal of retrieving and preserving the remnants of the culture before it was forever lost. 
IKTOMI: A CHARACTER TRAITS ANALYSIS

\section{Ethnography of stories.}

\section{Documenters' method.}

The ethnographers Zitkala S̆a, Deloria, and Walker collected and documented their stories around the turn of the century, spanning the years from 1896 through the 1920s. Walker started collecting materials around 1896, although only part of his work has been published, and that only posthumously; Zitkala S̆a published her first collection of stories in 1901, and Deloria first published Dakota material in 1928 and 1929.

The collections: Sources and approach to recording stories.

Zitkala S̆a began writing down the stories she remembered from her childhood to help counter her experience of oppression at boarding school. In her preface to Old Indian Legends (Zitkala S̆a, 1901/1985), she reflected on having heard the same stories repeated over time in different places by many different storytellers. Each time she heard a story retold, she had noticed variations that often provided missing information and helped connect the characters more clearly (pp. v-vi). Zitkala-S̆a vividly recalled the stories as she wrote, and she did not seem to need to rely on help from informants or interpreters to clarify meaning or fill in forgotten details.

This version of Iktomi (i.e., Iktomi and the Ducks) from her collection is the first

of five tales where Iktomi figures as the lead character; Zitkala S̆a selected these tales for inclusion based on what she perceived as wide appeal to children of all ages and backgrounds and to emphasize the particular indigenous values they transmit (Picotte, 1985, p. xiii). 
Deloria's Iktomi Tricks the Pheasants comes from a collection of stories told to her by Hunkpapa Lakota and Blackfeet informants on the Standing Rock Indian Reservation (E. C. Deloria, 1932, p. X). However, she was also known to work with other Native compilers of stories, such as the Lakota anthropologist, George Bushotter, and Oglala storytellers, George Sword (Long Knife), Luther Standing Bear, and Edgar Fire Thunder, all from the Pine Ridge Reservation. Deloria reworked earlier collections of texts originally documented in the Sioux dialects, such as those collected by Bushotter (Eichhorst, n.d., para. 6), transcribing and translating them into English. She worked in collaboration with Boas and many other noted scholars of anthropology at Columbia, such as Margaret Mead, Ruth Benedict, and Alexander Lesser. Boas had wanted to secure a variety of source materials to crosscheck against those Walker had collected and, recognizing Deloria's personal insight into the Dakota culture, he sent her to corroborate and authenticate the veracity of Walker's accounts. She translated copies of several of Walker's manuscripts and scrutinized his interpretations although she apparently sought out different storytellers than those with whom Walker had conferred in order to obtain different renditions of similar stories.

Deloria did not rely on her own authority but continually received guidance from her father concerning tribal customs and beliefs, as well as instruction from her anthropology mentors and aids at Columbia. Because of her unique combination of traditional Native upbringing and formal Western education and training, she was able, in effect, albeit inadvertently, to fashion a natural bridge spanning the cultural and intellectual divide between the White and Indian approaches to documentation. Her 
extraordinary vantage point resulted in an excellent repository of manuscripts and documentation of Dakota texts with English translations and interpretation based solidly on the Native perspective, while meeting the standards of academia and science. Her accomplishments include published fictional and non-fictional works depicting Dakota life, cultural practices, and values.

Walker's version of Iktomi comes from archival records housed with the Colorado Historical Society, copied from his own manuscript, which was contributed by his granddaughter, Mrs. Emeline Wensley Hughes after his death (Walker et al., 1983, pp. ix-x), and the American Museum of Natural History from notes and materials sent by Walker, himself, to anthropologist Clark Wissler.

A number of Oglala medicine men on Pine Ridge had inducted Walker into their society of traditional healers, and they instructed him in the Lakota language and customs, which included tribal stories. Two members of the group, George Sword (Long Knife) and Thomas Tyon (Gray Goose), who were, themselves, accomplished storytellers, acted as Walker's primary aids in documenting and translating stories and other cultural materials. In fact, Walker named Tyon as his informant for Iktomi, although, as mentioned earlier, the issue of authorship is unimportant in tribal society. Many people undoubtedly enjoyed easy familiarity with tribal stories since they were passed around so liberally. Consequently, the roles between storyteller, transcriber, and translator frequently overlapped and blurred into anonymity.

Although he could write Lakota, Walker never became fluent in the language and relied on others, most frequently Tyon, to translate his texts. Tyon often transcribed and 
translated not only his own versions of stories but also those told by other Lakota storytellers such as Left Heron (Makula or Hokacatka), Bad Wound, Little Wound, No Flesh, and George Sword, who all provided material to Walker (Walker et al., 1983, pp. 15, 23). Two White brothers, Charles and Richard Nines, fluent in the Lakota dialect, also provided him with translations and interpretations (Walker \& DeMallie, 1982, p. ix).

\section{Documenters'motivation.}

We really know very little about the daily conditions surrounding the family life of Iktomi's three documenters, which may have affected the development of their later opinions and motivations however, we can infer a few details from autobiographical and biographical sources.

Stated facts from a variety of sources already cited presuppose that Zitkala S̆a and Walker emerged from unstable home environments; Zitkala S̆a learned about abandonment through an absent father, while her mother's attitudes helped cultivate a burning resentment toward Whites. Apparent competition and rivalry among her siblings further exacerbated tension in her home, preparing fertile ground for Zitkala S̆a to be drawn away by tantalizing promises from strangers. Her subsequent experiences at boarding school only exacerbated her feelings of isolation and loathing toward those who wore down her sense of self and she emerged embittered and combatant, although she had also acquired a strict sense of discipline which she used as a defense. Yet, she had gained confidence about her talent and intelligence and found a way to integrate those qualities with her traditional values of community and selflessness to promote social change for the recognition and welfare and of her Indian people. 
Walker had grown up in a region politically divided over loyalties that became more pronounced as the Civil War progressed. Southern Illinois had been populated by first and second generation European immigrants and displaced sympathizers from the American South who had resettled in the fertile agricultural lands made accessible by a network of major water routes. However, Illinois had aligned with the northern states in the conflict, which set ideologies at odds with one another and disrupted family cohesion.

Walker led an indistinct life as a child among his many siblings, and as the eldest, he possibly felt undue pressure and responsibility toward the others. Further, he may have wished to escape an early expectation to carry on the family farm in order to pursue a career of his own choosing, and the provocative political situation provided his release. His brush with illness in the military may have sparked a covert interest in healing, leading him to pursue an enthusiasm for medicine, particularly regarding curing and eradicating infectious diseases. His inquisitive nature propelled him toward the secret practices of the Native medicine men who also recognized his avid interest and dogged perseverance in curing the sick. Very likely, they also fulfilled a fatherly role for Walker, who had lacked paternal support and guidance during his critical formative years.

The elder Native men took him under their wing and, in return, Walker dutifully recorded their instructions and provided them with a voice that would carry their message into the future. Editor of Walker's manuscripts Jahner surmised that the old men likely knew their ways would soon die out and sought a way to preserve their cultural practices and ceremonies for their future generations. Without the ability to practice their culture, they realized written documentation was the only alternative, and they found in Walker a 
receptive pupil and advocate (Walker et al., 1983, p. 16). Walker honored his mentors' request, writing everything he could just as they dictated in order to preserve the cultural knowledge in their own words and from the Native's perspective. However, Walker's motives may not have been entirely altruistic; he desired to know all he could of Native practices for his own satisfaction and he occasionally interjected his own interpretations into the text, muddling the Native clarity of his informants' comments. Wissler's expressed interest in obtaining Lakota materials also sparked Walker's enthusiasm; whether the notice fed his passion for learning or Walker anticipated additional benefit from projected results is not apparent. Nevertheless, Walker succeeded in compiling a massive quantity of raw material which has given us more than a glimpse into the old Dakota culture.

Despite the difficult environment surrounding her people, Deloria enjoyed a more stable, structured family life, solidly based on strong faith and principles. She received substantial support from her family, and her father laid out a clear path for her to follow, beginning with attending the school he had founded. Deloria possessed a strong sense of belonging; she knew who she was and where she fit into the family and community structure. Picotte (1988) described Deloria's family as "a large and loving one”, well grounded in its Episcopal religion as well as traditional Dakota culture and values (p. 229). Due to the respected stature of her family, Deloria did not have to grapple for identity and place but found it securely within the household where family was given top priority. Consequently, the stability that enveloped Deloria very possibly fostered her devotion to her family and her great empathy for the plight of others. She combined a 
sense of the Dakota value of the giveaway and the Christian value of forgiveness to promote better relations between Whites and Indians, which she expressed through her attempts to increase Whites' awareness and understanding of the Indian as a human being (DeMallie, 1983, p. 237). She found a natural fit with Boas's approach to cultural anthropology and strove to enlighten and clarify for the Whites as much of her Indian culture as possible. Throughout her life, Deloria held her altruistic belief that better understanding could truly change attitudes and improve relations, whereas ignorance had fostered only hostility and oppression.

Thus we observe three very distinct individuals who followed their own course and for their own individual reasons produced their unique version of this Dakota ohuykakay myth. Each sought to fulfill a desire to save a small portion of a dying culture and overcame incredible hurdles to preserve their scraps of a "primitive" literary artifact. Although the tales were discounted by the greater Western society as mere children's stories (Mead, 1932/1969, p. 82; Reichard, 1938, pp. 477-478), these three documenters apparently suspected the presence of the riches that lay buried among those scant words.

\section{Part 3: Pronouns and Enclitics}

In this section of the literature review, I turn my focus to another element of structure themed throughout the Iktomi story that merits attention, that is, the prevalence of pronouns referencing the masculine aspects of the characters. The crux of my thesis centers on an assertion of male focus in the Iktomi story, and to that end, indicators of biological gender abound in Iktomi, such as kinship references indicating sex of the characters, direct mention of female ducks, and the nature and gender appropriateness of 
the activities the various characters engage in. I will elaborate further on the significance of these important themes in Chapters 4 and 5.

To maintain the assertion of male orientation in Iktomi and further support my thesis that this Iktomi story represents a framework for a male rite of passage, I needed to address the use of pronouns incorporated in the story, particularly with regard to indicators of sex of the characters and masculine and feminine qualities and traits they exhibit. I present here a brief overview of the Dakota pronoun paradigm as well as enclitic devices used to indicate sex of speech participants as they relate to the Iktomi story. Then, in Chapter 3, I describe the process I used to ascertain how the pronouns and enclitics are used in the story, followed by a discussion in Chapter 4 of results and findings from my analysis of their use with specific relevance to indicating the sex of the characters.

The aspect of data analysis regarding pronoun usage in this study draws on basic concepts of a stylistic approach, whereby literary texts are analyzed based on the frequencies of keywords and/or frequent phrases that occur in the text. An analysis of frequently occurring keywords, in this case, English pronouns, provides additional information about the characters' motivations and intentions, which might not be readily discernible from their actions alone. The use of certain types of pronouns can reveal subtle relationships between emotional and functional elements that co-occur in the text, and when applied to pronoun usage in Iktomi, the relationship ties between the characters became more pronounced. Analysis of pronoun usage in Iktomi helped, among other things, to definitively identify the characters, to establish the relationships between 
characters, to determine the strength of the characters' roles relative to the main theme of the story, and to provide insight into the intentional motivation of the primary characters and ascertain deeper meaning from the story's message.

\section{Dakota Pronoun Paradigm: Grammatical persons and number.}

My concern for accurate evaluation of textual content necessitated an assessment of existing correspondences between Dakota and English usage of certain grammatical structures since I was working with English translations of Dakota texts. To assure grammatical consistency, I consulted several early and recent Dakota language dictionaries, grammars, and studies to obtain comparative information for sorting out parallels between the Dakota and English system of markers of sex and gender, particularly regarding personal pronouns and enclitics, which I discuss in the next paragraphs.

The grammatical person and number system in the Dakota language roughly corresponds to that of English, incorporating first-, second-, and third-persons in subjective, objective, and possessive case. Dakota also incorporates a dual number in the first-person subjective case only, encompassing the dual you and I as the speaker and the person spoken to. According to the New Lakota Dictionary (NLD), D/Lakota third person singular is unmarked for gender, while third person plural distinguishes nouns between animate_- "people and animals of all sorts"—-and inanimate_-"non-living things and abstract concepts." Third-person plural pronouns for animate nouns include distributive plural and collective plural where distributive refers to they as individuals- 
each of them - and collective refers to they as a collective group or a general collective state or activity (Ullrich \& LLC, 2011, pp. 757-760, 764).

$\mathrm{D} /$ Lakota uses pronouns in the form of personal affixes attached to the verb to denote first and second person, including dual inclusive. Unlike the English system, Dakota third-person-singular pronouns do not distinguish between the masculine, feminine, or neuter (i.e., he, she, it), that is, gender is not marked. Third person singular is implied through the citation form of the verb while third person plural is marked using post-verbal enclitics. Plurality for animate nouns is marked using the suffix $-p i$ for distributive nouns and the affix -wicha for collective nouns. Inanimate nouns are not marked for plurality on active verbs but are understood from context or through the use of demonstratives or stated numbers. For inanimate nouns with stative verbs, plurality is accomplished through partial or total verb reduplication (Ullrich \& LLC, 2011, pp. 757$760,764)$.

\section{Dakota enclitics as indicators of mode and gender.}

Since the foregoing discussion of Dakota grammatical persons precludes identification of the speaker's sex through pronominal use, another means of determining the sex of the story characters had to be applied. The NLD notes the Dakota language employs enclitics as such devices that "follow verbs,... usually cannot stand alone,... [and] express such grammatical features as tense, modality, duration, aspect, etc." The NLD adds that "in a few cases, men and women use different enclitics" to denote interrogative, declarative, and imperative sentences (Ullrich \& LLC, 2011, pp. 820-821). 
Early mention of enclitic devices is found in Riggs's (2004) Dakota Grammar with Texts and Ethnography, which was first published in 1893. Riggs remarked about only those enclitics that serve as imperative mode indicators, referring to them as syllables which attach to the end of the verb. In a similar but later context, White Hat, Sr. described similar features as "gender endings...that end a sentence and identify the gender of the speaker" (italics mine; Kampfe, 1999, p. 18) although he included forms that also relate to mood, that is, statement-, question-, and command-type sentences. Trechter (1996) presented an in-depth study on deixis in the D/Lakota language in which she focused specifically on enclitics, as she also termed them. Her study affirmed very specifically that certain enclitics denote sex of speech participants as well as other nuances that get expressed through the communication event such as relative age and stature of the interlocutors, emotional content imparted through the utterance, level of authority held by the speaker, power balance between the speaker and the addressee, and whether the spoken words issue from direct or indirect knowledge (pp. 100-116). ${ }^{6}$ In summary, what Dakota grammars term enclitics are post-verbal particles that provide information about stance broadly conceived.

I conclude my review of the literature, having discussed of a variety of factors that played a role in shaping the versions of the story under examination in this study. To recap the material provided in this chapter, Part 1 dealt with aspects of social theory and scholarly approaches to Native American myths that are relevant to my focus on storytelling in the Dakota culture by supplying the sociopolitical backdrop to

\footnotetext{
${ }^{6}$ Trechter (1996) provided a thorough discussion of Dakota gendered enclitics, including academic and native study and commentary on their nature and use. Her sources include Dakota stories, conversation, songs, dictionaries, and grammars.
} 
contextualize the telling of the story in real-world time and place, including the story's role in Native culture. I also backgrounded earlier work by scholars studying Native storytelling processes, including debate and critique of organizational frameworks and approaches posited and used by pioneers in the field. Part 2 attended to factors surrounding the ethnographers and informants that may have influenced the various renditions of the story in order to give relevance to the different versions and humanize the storytelling experience through which each version emerged. Finally, Part 3 of this chapter provided a brief, partial overview of the Dakota pronoun paradigm that compares with the intended meanings of pronoun and enclitic usage carried over into the English translations. This information acknowledges differences in grammatical structure between languages that may have affected the degree of faithfulness of English translation from the intended meaning in the original versions rendered in Dakota. In the next chapter, I present the methodology I used to analyze the three versions of the story Iktomi. 


\section{Chapter 3. Methodology}

In this study, I conducted a qualitative exploratory analysis of three separate English language versions of the Dakota (Sioux) folk myth Iktomi, with the primary focus on character portrayal. I compared and contrasted textual content to ascertain patterns as well as consistencies or inconsistencies among the different versions that became evident through analysis of coded traits as they relate to the characters in the story. As noted in the literature review, I used the three versions that were documented by Zitkala S̆a (1901/1985), E. C. Deloria (1932), and Walker (Walker, Jahner, \& SHSC, 1983).

I have organized the methodology chapter into a number of sections beginning with a brief introductory background of the story used in the study. Background information covers the story's cultural context, materials and data selection, the layout of synoptic alignment I used to compare the texts, and preliminary character identification. Following the introductory overview, I include sections describing my data coding procedures, which cover the three phases of data coding: (1) character portrayal; (2) polarity-code correspondences; and (3) pronoun usage. Subsequently, I briefly describe my approach to assessing documenter motivation and finally, I conclude the chapter on methodology with a section describing the calculations I used to arrive at quantitative measures for analyzing the text data.

\section{Background and Context}

Recognized under various titles, the story Iktomi is a well-known traditional folk myth among the Dakota people. For the purpose of identification and record in documenting collected stories, translators would typically assign a working title to their 
respective versions of the texts. Zitkala S̆a titled her version Iktomi and the Ducks;

Deloria called her version Iktomi Tricks the Pheasants; and Walker's version was named, simply, Iktomi. For the purposes of this paper, I have retained these titles in the data though I will reference them in two different ways in the discussion: (1) I will use the generic Iktomi as a generic reference to the story, regardless of the version; (2) where further distinction of a particular version is otherwise necessary, I will use the last name of the corresponding documenter as the identity reference for the specific version indicated. Thus, the distinguishing references will be Zitkala $\breve{S} a$, Deloria, and Walker, respectively, for Iktomi and the Ducks, Iktomi Tricks the Pheasants, and Iktomi.

The Iktomi story has been retold countless times throughout the history of the Native people who have shared it with various audiences of all ages in the customary tribal practice of oral storytelling. In accordance with Dakota tradition, it has been used to pass along cultural values and moral teachings from generation to generation as well as to entertain and instruct members of the societal group. It is because of this free transmission of the story that variations have come about, and the titles have been assigned only arbitrarily as a means to identify a given segment or version of a larger collection of episodes from which a storyteller could choose a theme to convey a particular message. Ordinarily, Dakota storytellers did not give the stories titles because of their mutable nature, and, as indicated above, the titles were attached by the documenters who worked with written texts. 


\section{Cultural context.}

To help orient the reader and facilitate better understanding of the story's basic role in the Dakota culture, a brief description of the organization of Sioux story types is useful to show how the tale fits within the cultural framework. In the introduction to Dakota Tales, E. C. Deloria (1932) provided the organizational backdrop for readers with her description of how the oral literature genres of Dakota folklore are categorized from the Native perspective. The Dakota system classifies cultural stories in two main divisions, which are subdivided into four distinguishable story types. Figure 1 illustrates the schematic organization of these four types.

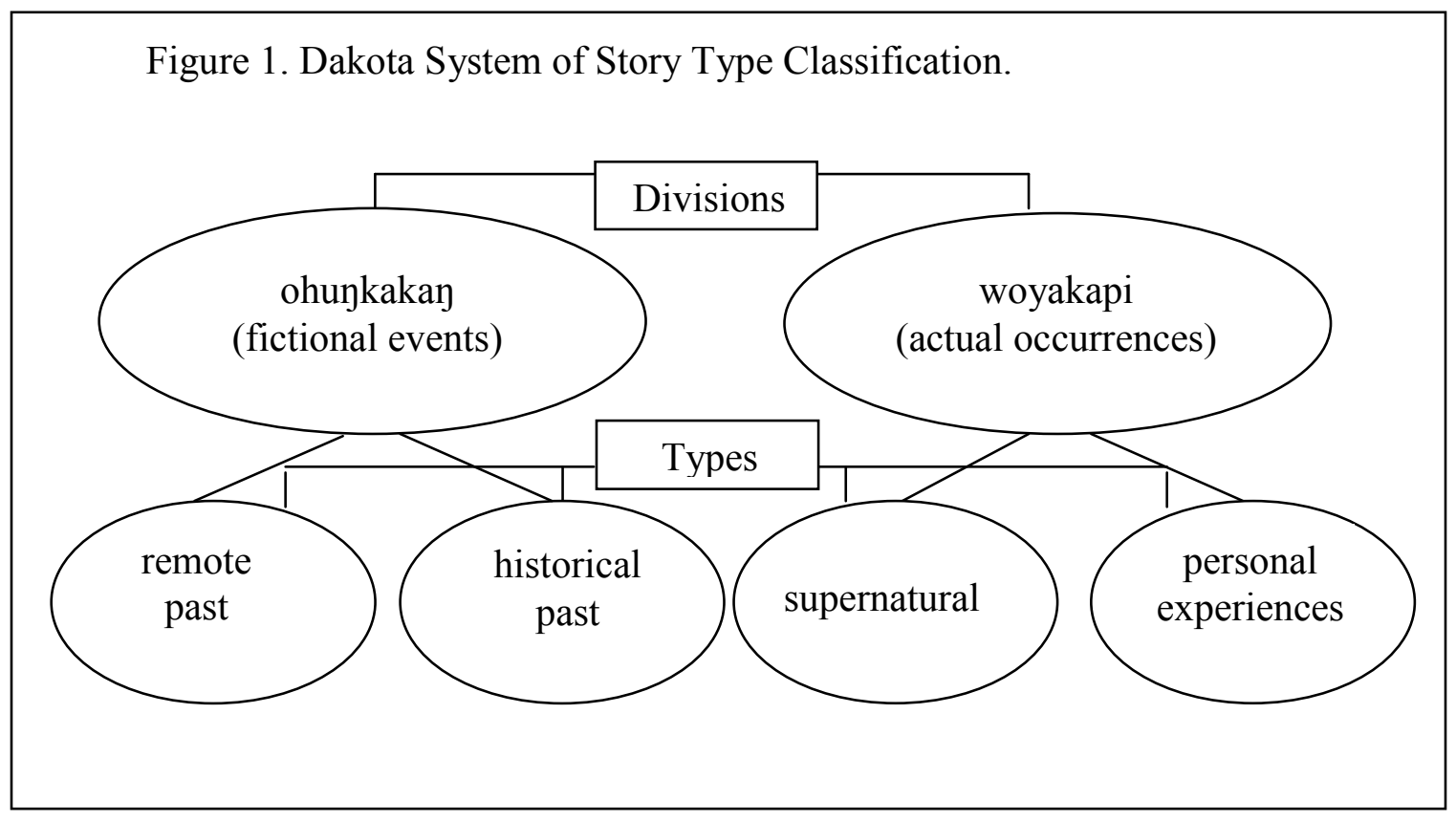

The first division contains the mythological ohuykakay tales, which are accounts of past fictional events artistically constructed to amuse, entertain, or teach an audience. The ohuykakay are subdivided into two groups: one subgroup includes stories that are situated in the remote past, having occurred during the pre-human era; the second group 
includes tales or accounts of more recent events that occurred in the historical past though are fantastic, incredible, and magical in nature. Both of these types of stories involve events that seem improbable to have actually occurred in anyone's personal experience.

The second major division of stories contains the woyakapi, which include accounts of events that have actually taken place at some point in time in the human experience. These stories are also arranged into two subgroups: the first subgroup of the woyakapi includes actual events that involved intervention by supernatural powers; the second subgroup includes stories of actual events that took place or happened to a person within the experience of the family group or larger society. Both subcategories of the woyakapi assume the events occurred relatively recently in the history of the tribe, perhaps to a grandparent or great-grandparent.

The particular folk myth Iktomi used in this study falls into the category of the first subgroup of the ohuykakay tales; the story takes place in the remote past, seems improbably cast with animal characters that assume humanlike identities and characteristics, and imparts a moral teaching in the expression of performed actions and their consequences through a series of actions and dialogue (E. C. Deloria, 1932, p. ix).

\section{Selection of Materials and Data Collection.}

As noted in Chapter 1, I did not feel content with the strategies and methods that have been used thus far for studying Native myths, and consequently, I have sought new techniques for the systematic analysis of Native American stories, especially with regard to Dakota folk myths. 
First, instead of working with one version of a single story, I have chosen to compare three versions of the same story to discern what, if any, differences might exist among them. I used the translated English texts of three different versions of Iktomi as the data sample. All three versions originate from separate individual narrations of the Dakota ohuykakay cultural myth, which have been made available in three distinct existing works in published form (cf. E. C. Deloria, 1932, Walker et al., 1983, and Zitkala S̆a, 1901/1985). All the versions were originally narrated orally by fluent native speakers of the Dakota language, and two were transcribed and translated into English during the late $19^{\text {th }}$ or early $20^{\text {th }}$ centuries; Zitkala S̆a wrote her original version in English. I used only the English translations here to maintain consistent uniformity in the analysis since Zitkala S̆a's version is not available in the native language.

Working without a clear guideline and no prior model, I intuitively decided to focus on characterization to discern deeper cultural meaning embedded in the myth. I sensed the story's characters might carry the most significant import in terms of a cultural message that might be woven into their actions and interactions. I drew inferences from the texts since, in Walker's version, the peripheral detail was very scant and unlikely to offer very much information. I surmised that if the stories were essentially equivalent, such content information might not produce satisfactory results.

I then turned to the characters and made a quick determination that all of the versions consistently employed the same players. Frustrated with the surface reading of the story and reluctant to settle on simplistic discursive meaning in the actions of the characters, I purposed to approach characterization from a different angle. I wanted to 
get to the very heart of the characters and find out what they were made of and what motivated them in a way that would speak truth to a Dakota listener. To ascertain this level of information I elected to dissect the characters and conduct my analysis on their traits, trait patterns, and relationships that may exist among the traits. I will return to characterization shortly.

\section{Synoptic Alignment.}

For the purpose of comparing textual content among the three versions, I synoptically arranged the three texts in a grid using the Table function in Microsoft Word 2007 to align their respective storylines relative to one another as the tale unfolds. Only one of the original texts—E. C. Deloria (1932) — was rendered with assigned line numbers, so I used that text as the baseline for aligning the other two. I took care to retain the sequential order of story progression within each of the texts even though the order of specific related or identical events varies somewhat in detail or presentation among the texts. This method of organizing the texts preserved the validity of the data while I searched for patterns that might emerge resulting from the sequencing of story details. I include a sample of the texts arranged synoptically in this manner as Appendix B.

Additionally, as shown in the appendix, I divided the individual sentences into smaller units of detail to isolate identifiable fragments of the narrative which depict unique character traits, qualities, or actions that could illuminate similarities or differences from one text to another. I identified the individual components, thus segregated, with alphabetic indicators marking them as subsets of the numbered lines to 
facilitate recognition of the correspondences between texts. I also cross-referenced the line subsets wherever discrepancies occur in the story progression to aid in tracking the events and incidents as they occur or are omitted from one version to the next.

\section{Preliminary character identification.}

A preliminary step to coding the data is to identify potential characters in the story. Although it might seem a straightforward task, I sought to make no assumptions based on a Western literary framework of characterization which typically includes only conscious living entities, that is, humans or animals. In ohuykakan tales, animals, plants, and even inanimate objects can take agential thematic roles and engage in conscious or reasoned activity. Likewise, again from the Dakota frame of reference, elemental entities, such as wind, fire, water, and mineral substances such as stone, often possess powers and abilities similar to those of their human or animal counterparts. Therefore, I anticipated that the resulting cast of characters to be analyzed could change from my initial determination and that some identified characters might be eliminated if the coding showed their role and involvement to be inconsequential to the themes and patterns that might emerge.

Based on apparent thematic agency, I isolated a preliminary list of seven potential characters to consider in the coding process. Reading through each of the texts, I considered each element of animal, plant, mineral, and elemental that seemed to exhibit volition, mobility, or the ability to act or cause an effect on another element in the story and included them as possible character candidates. These seven character candidates 
include (1) Iktomi, (2) the Ducks, (3) Skiská ${ }^{7}$, (4) Wind, (5) Tree(s), (6) Wolf/(ves), and

(7) Fire since each appears to display some type of active role and/or interacts with at least one of the other character candidates.

\section{Data Coding Procedure}

I incorporated three different approaches to coding and analyzing the data. In the first phase, I focused on character portrayal and coded the texts for character traits, qualities, and actions. In the second phase, I arranged the coded traits into subsets of polarity-code correspondences. In the third phase, I looked at the use of pronouns and how they might provide additional insight into the nature of the characters and their motivations.

By means of an inductive process, I began to devise my coding method by developing codes for character traits. Initially, I worked through the story line by line and assigned a code to each feature as I identified it. Because of the exploratory nature of the study, I exercised wide flexibility and expansiveness to avoid forcing the data into any preconceived notions of content, form, or description. This process produced three emergent categories relating to representation, abilities, and attributes exhibited by the various characters. The coding initially yielded 13 codes for representation, 34 codes for abilities, and 64 codes for attributes, each of which pertained to one or more characters. Then, by analyzing relationships between the coded traits, I was able to reduce the original number of codes to six codes for representation (Rp1-Rp6); four codes for abilities (Ab7-Ab10); and five codes for attributes (At11-At15). These are the codes I

\footnotetext{
${ }^{7}$ Skiská is the Lakota term for "wood duck."
} 
used to analyze the data. I have provided a synopsis of the codes and their descriptions in the Character Traits Coding Template in Appendix C.

The data coding method thus developed as a top-down plus bottom-up complementary reiterative process through trial and error whereby the traits coalesced into general categories, the emergent categories drove identification of more traits, and the traits, thus identified, helped narrow down and refine the broader categories.

I used these 15 codes along with their polarity correspondences, which I describe in Phase 2 below, to discern patterns among the traits of characterization and any relationships between them that might yield insight into the cultural message that I anticipated to exist in the text.

\section{Phase 1: Character portrayal through traits.}

In this section, I describe the categories as they relate to aspects of characterization that helped me narrow the cast of characters and define them and their relationships to each other.

The three categories group the characteristics according to aspects of identity, consciousness, ability, and innate quality as exhibited or expressed through character activity and interaction. Briefly, the category of representation includes such traits that are descriptive of the manner and forms by which the characters are referred to or addressed by the narrator or other characters and also by the personifying traits they exhibit, i.e., whether they are able to determine or influence their fate. For example, representational forms identify the characters as living versus non-living entities and as human versus nonhuman. The category of abilities includes physical, sensory, rational, 
and locomotive or mobility traits or activities that the characters exhibit as they interact with their environment, i.e., how someone — another character — sees or responds to them. Examples of character abilities include walking, crying, reasoning things out, manipulating objects, and so forth. I determined the third category of character attributes from the way the characters are presented in situational context, how they are experienced by each other, and how they relate to and interact with one another and with themselves. Attributes include personality traits, behaviors, attitudes, and the like. Later in this section, I will expand my discussion of the three main categories to more clearly establish the distinctions between them. First, however, more descriptive detail with regard to the codes will help the reader better ascertain those distinctions.

\section{Categorization of traits.}

In this section, I describe the system I used to classify the different types of character traits into the more narrow subcategories. Although I have identified and only loosely defined the main categories, I begin my description here with the subcategories of traits codes within the context of the three main categories, since the subcategories offer more definitive detail that will facilitate understanding of my rationale while sorting the traits. Then, I describe the code construction in order to illustrate the relationship between character identity, character traits, and traits types. I follow up this discussion with clarification about what constitutes the primary categories and distinguishes them one from another.

As described earlier, I condensed the large number and variety of codes derived in the coding process into the 15 subcategories within each of the three primary categories 
of classification. I reduced the number of codes in order to minimize their variability and to bundle them into fewer manageable and quantifiable groups. Working with a smaller number of subcategories also facilitated consistency in assessing any emerging thematic patterns that arose within individual versions or appeared to correspond between versions. The resulting 15 codes are arranged by category and subcategory in Appendix $\mathrm{C}$ along with brief generic descriptors linking them to character traits found in the texts.

\section{Category: Representation.}

As can be seen in Appendix $\mathrm{C}$, the main category of representation includes six subcategories labeled: Rp1:Nonhuman; Rp2:Human; Rp3:Pronoun-male reference; Rp4:Pronoun-other; Rp5:Recognition; and Rp6:Powerlessness. The nonhuman subcategory includes references that indicate the subject is not human, though it may be animal, plant, mineral, or elemental. The human subcategory includes traits that may be suggestive of human characteristics, including references to hands, speaking, cooking, and other such indicators that would not be suggestive of an animal (or plant, mineral, or elemental, for that matter). The third and fourth subcategories of traits comprise personal pronouns used in the texts. I included the use of pronouns as a possible source of significant information that might reveal insight into the nature of individual characterization by the narrators. I made the assumption here that the third person singular masculine pronouns could prove significant since the story reads as though the title character Iktomi is personified as a male entity. Consequently, I separated that group of pronouns into its own subcategory of pronoun-male reference and grouped all other uses of personal pronouns into a separate subcategory of pronoun-other. The 
subcategory of recognition incorporates references of personal address and acknowledgement of the characters by other characters. These references include using a character's name or kinship term as differentiated from impersonal references that do not acknowledge any type of personal identity of the character. I included the final subcategory of powerlessness in this main category of representation to accommodate aspects of character that seem to further refine the identity and personification of the subject in terms of personal effectiveness and being in the world. For example, Iktomi's powerlessness stance is indicated by his inability to free himself when he is caught in the tree; further, while caught in the tree, Iktomi is powerless to stop the wolves from eating his roasting ducks.

\section{Category: Abilities.}

An additional four subcategories comprise the primary category of abilities and are labeled: Ab7:Skills; Ab8:Sensory/rational/emotive; Ab9:Locomotion; Ab10:Willful intent. The skills subcategory includes abilities to manipulate objects or perform specialized tasks, such as working with tools, building a fire, or constructing a house. The Sensory/rational/emotive subcategory includes traits related to the senses, perception, and character self-expression. Some examples of these traits include smelling, thinking (about how to do something), crying, and feeling (e.g., fear or alarm). Locomotion refers to the character's ability to move or relocate under its own power. Instances of locomotion can include walking, flying, and leaping, among others. The subcategory willful intent includes character ability to use purposeful design to commit an action or accomplish an objective, often premeditated and directed toward someone or 
something else. Examples from the data include Iktomi's killing of the Ducks, the Wolf/(ves)' eating of the roasts, and Iktomi's placing a curse on the Wolf.

Category: Attributes.

I subdivided the primary category of attributes into five subcategories, labeled:

At11:Appearance/personality/inclination; At12:Disposition; At13:Behavior/attitude

toward others-negative; At14:Behavior/attitude toward others-positive; and

At15:Egocentrism. The subcategory Appearance/personality/inclination includes

references to innate characteristics that are inherent to the character as an individual, including personality and physical traits and natural mannerisms. The subcategory disposition refers to natural impulses of emotional or mental aspect, such as wiliness, mischievousness, conceit, curiosity, and so forth. The two subcategories behavior/attitude toward others-negative/positive include similar types of traits, one unfavorable and the other favorable. Unfavorable negative traits include vanity, exclusion of others, demanding behavior, and refusal to cooperate. Favorable positive traits indicate a character's inclusiveness and willingness to identify with others, such as courtesy, concession, and agreement. The last subcategory egocentrism involves traits indicating self-centered behaviors and attitudes, such as self-pity, possessiveness, stinginess, and condescension. The distinction between the behavior/attitude and egocentrism subcategories lies in the interactive nature of the traits. Behavior/attitude toward others implies interaction between characters whereas egocentrism is inwardly focused. 
IKTOMI: A CHARACTER TRAITS ANALYSIS

\section{Components of data code construction.}

Having identified the potential characters and sorted their 15 different types of traits into primary categories and subcategories of classification according to their similarities, I constructed unique traits code identifiers that I used to quantify and tabulate the data. The 15 unique data codes are constructed in three parts consisting of alphanumeric identifiers that correspond to the three components of character, category, and subcategory of traits.

\section{Character identifiers.}

Character identifiers include IK, DU, SK, WI, TR, WO, and FI, and respectively represent Iktomi, Ducks, Skiská, Wind, Tree(s), Wolf/(ves), and Fire. The character identifier links the specific character relationally with the category and traits indicated by the remainder of the code.

Category identifiers.

Identifiers of category include $\mathrm{Rp}, \mathrm{Ab}$, and At, and correspond to the three categories of representation, abilities, and attributes, respectively. These identifiers sort character traits, qualities and actions into broad divisions by type, such as the type of entity as it is presented by the narrator or referred to by other characters (representation), what the character is able to do (abilities), and its innate characteristics (attributes). The category identifier attaches to the character identifier and, therefore, links the corresponding trait to the specific character. 
Subcategory identifiers.

Subcategory codes are indicated numerically, as described in the preceding section. The numeric identifier for a specific trait, quality, or action affixes to the end of the category identifier and serves to group related aspects of character into subsets of the broader categories whose correspondences have already been linked to a particular character.

To illustrate the construction of the codes, refer to the text sample in Appendix B and note the text in line $3 i$, which reads:

$$
\begin{array}{ll}
\text { Zitkala S̆a: } & \text { as he staggered along } \\
\text { Deloria: } & \text { and then walked past } \\
\text { Walker: } & \text { and ran past the ducks. }
\end{array}
$$

The verbs staggered, walked, and ran refer to actions performed by Iktomi, so I assigned the first part of the code the character identifier $I K$. The action of the verb indicates ability; therefore, I assigned the second part of the code the category identifier $A b$. The active verb denotes locomotion, so I assigned the third part of the code the subcategory identifier number 9. The code assigned to this verb results in IKAb9.

No additional reference to the individual version of the story is needed in the code, since I am not comparing the stories for textual constants. It suffices to mark individual occurrences of traits whether or not they occur in a particular version. Once all the traits were coded, I tallied their number of occurrences in each separate version, and I was able to create a profile for each of the characters as the individual narrators portrayed them based on the composite of all of the coded traits for their respective 
versions. I then used the character profiles to make comparisons between texts and examine the coded details for patterns and inconsistencies.

\section{Category distinctions.}

Returning now to the three primary categories, I explain how they emerged in congruence with the coded traits. I recap the descriptions of the categories followed by an explanation of how story characters derive their traits. From there, I more fully elaborate on what constitutes the three primary categories and conclude by describing the nature of traits and their links to the categories.

Synopsis of categories.

Briefly outlined, the representation category, subdivided into six descriptive subcategories, attempts to capture references that point to character identity as a living or non-living entity, and more specifically as nonhuman, human or humanlike, as well as how the characters relate to self and others through anaphoric reference (pronoun usage) and relative stance (powerlessness). Traits in this category give us a sense of the intrinsic form of the character without personalizing detail about its capabilities or tendencies, although we do ascertain something about its relationship to other characters. The abilities category segregates qualities according to subcategories of cognition, sensation, action, and will, which enable comparison and contrast of traits of different characters by comparable measures and provide more bases for typing characters and assessing their relationships. The attributes category groups traits according to naturally occurring qualities and inclinations of personality, conscious and unconscious impulse, conditioned or learned behavior and attitude toward other characters, and self-directed behavior and 
temperament. As with representation and abilities, attributes enable logical comparisons between characters to determine patterns of relationship and discern consistencies or discontinuities between the three different versions of the tale.

In classifying the codes within the categories and subcategories, I used specific references from the data as defining criteria for each of the codes and sorted them by type and according to the relationships I perceived to exist between them. As mentioned, I culled these references during the process of condensing the original set of codes into the final 15 character traits codes. Occasionally, certain coded traits were grouped into a particular category or subcategory that they do not seem to directly relate to well. For example, I coded the act of "eating ducks" as an ability of willful intent whereas it could also possibly be considered a skill. I made coding choices based on the best fit between the individual trait and the other traits within the particular subcategories using the detailed descriptions from the template (cf. Appendix C) as my guide, and the disconnect in relationship that occasionally occurs between traits thus transpires on account of the different levels of detail used to evaluate the traits.

Restated, my first approach to the coding process involved identifying character traits and grouping them loosely according to similarity of their qualities. Then, based on these initial determinations of relationship, I determined an appropriate descriptor for the group of similar traits which sorted into the categories and subcategories according to how they related to each other at that level of detail. Eventually, the descriptors coalesced into a group to which I assigned a distinct code. While many of the traits seemed to fit naturally into one category or another, occasionally, the choice between two 
possible categories proved a difficult one and I had to decide based on my best determination of the trait's relationship to others in the category. As stated earlier, the process of identifying traits and formulating categories occurred simultaneously in mutually complementary fashion. As I worked, I coded traits into loosely described categories, which in turn helped define and narrow the subcategories used for sorting the traits according to their similarities and relationships.

\section{Derivation of traits.}

Traits afford dimension to character figures and impress personalizing and differentiating characteristics upon them to various degrees. Traits may be inherent or ascribed, and they arise in the context of storytelling from two separate levels of discourse, one from outside the story in the world of the listeners, and the other from inside the story world as part of the storyline. First, as architect of the story, the narrator ascribes all the traits and qualities to the characters and uses them to sculpt the characters into their particular forms, giving them certain capabilities and assigning them indications of personality and individuality. The narrator provides character detail external to the story world, that is, information that informs the listeners about the characters but which does not come through the events occurring within the story itself. At the story level, the individual traits also emerge in the course of the unfolding tale, either through the narrator's own voice, the character's voice, or the voices of other characters. This trait information evolves from content internal to the story, that is, from a perspective inside the story world through the activities of the characters and events that progress through 
the plot. Thus, traits arise both through descriptive narration external to the storytelling setting and as part of what takes place from within the world of the story itself.

Types of traits.

With regard to the texts used in this study, character traits generally fall into three types: (1) representational, (2) demonstrative, and (3) innate. Traits and qualities may take an expected form, as through common knowledge, or an unexpected form assigned through one of the three voices, i.e., expressed through the narrator's voice, the character's voice, or the voices of other characters.

Representational traits define the character's form and its stance relative to other characters and conditions in the environment. These traits serve to create a particular figure presented in a particular form and to order the relationships among the different characters. Hence, I use the category label representation to organize these types of traits. Representation includes objectification of the character described by the type of figure the narrator portrays it as, and by how other characters perceive it and position themselves in relation to it. Character traits grouped in the representation category function to situate the characters along a continuum of personification ranging between the extremes of human and nonhuman (e.g., ducks which are birds and also speak), describe characters' relational status in comparison with other characters and props through anaphoric reference (i.e., use of personal pronouns), and position the characters according to relative stance according to the balance of power (e.g., Iktomi's inability to protect his roasting ducks from the Wolf(ves). 
Demonstrative traits display learned or inherent capabilities that show how characters operate and interact with the environment. Traits of this type indicate a changed condition, whether through the act of doing something (e.g., beating a drum), thinking through a process (e.g., devising a scheme to catch ducks), experiencing a feeling or emotion (e.g., alarm upon discovery that Iktomi is killing one's fellow ducks), expressing oneself (e.g., crying, speaking, pleading), or effecting a change in another character's state of being (e.g., killing ducks, that is, causing them to die; placing a curse to cause eyes to turn red). Because demonstrative traits indicate transformative quality, I have grouped these types of traits in the category labeled abilities. Abilities involve qualities that describe what the character is capable of doing or experiencing via mental, emotional, physical, and sensory processes. They include the faculties enabling the character to learn and reason things out (e.g., find the cache of roasting ducks), employ various modes of self-expression (e.g., cry, speak, plead), manipulate objects (e.g., use a drum beater), physically move (e.g., walk, dance), and perceive environmental impulses through the natural senses of sight, hearing, smell, touch, and taste.

Innate traits emerge as an aptitude or propensity inherent to the character's way of being. Adjectival and descriptive in nature, these types of traits do not, in and of themselves, result in effecting a changed condition on anyone or anything. Innate traits are unconscious tendencies and idiosyncrasies that may influence a character's actions; however, the characters do not actively discuss, reflect upon, or directly challenge them within the story. I have grouped these types of traits into the category labeled attributes, as they describe inherent characteristics that further color our perception of the character 
as an individual entity. Attributes include those traits an individual has no control over such as physical features, personality, temperament, and mannerisms, yet they also include learned or conditioned qualities that shape an individual's self-image and disposition (e.g., conceit, courtesy). Attributes also direct the character's behaviors and attitudes which we may think of as having been molded by conditioning through past experience even though, in the context of the story, the character has no past. A member of the audience might be able to relate to such past conditioning, however.

The traits and qualities of character are designations of metaphor or symbol (as discussed in Chapter 2) whose purpose serves to help the audience personally identify with the characters. The symbolic links may directly correspond coincidentally or uncoincidentally to specific human traits possessed by audience members, or they may indirectly allude to some generally recognizable aspect of character or behavior that listeners can nevertheless relate to or imagine as a possibility of cultural truth.

\section{Summary of data coding procedure phase 1.}

To consolidate the various steps involved in the data coding development process into a concise progression that is easier to conceptualize, I have placed them into a linear flow chart which is shown in Figure 2. The steps as they flow through the chart include:

1. Using the 3 texts synoptically aligned,

2. Identify the character quality or trait;

3. Categorize the trait according to its type;

4. Assess the trait's relationship to other traits within the same category;

5. Group traits of similar quality into numbered subcategories; 
6. Create individual codes by combining the signifiers for character, category, and subcategory;

7. Code the text: Assign individual codes to each identified character trait.

The recursive aspect came into play through the traits identification and coding process; in working through the text, newly identified traits occasionally surfaced that required a reassessment of my determination of categories and subcategories. As I indicated earlier, the entire process was not, in fact, linear, but required many back-andforth steps as I fine-tuned my codes. 


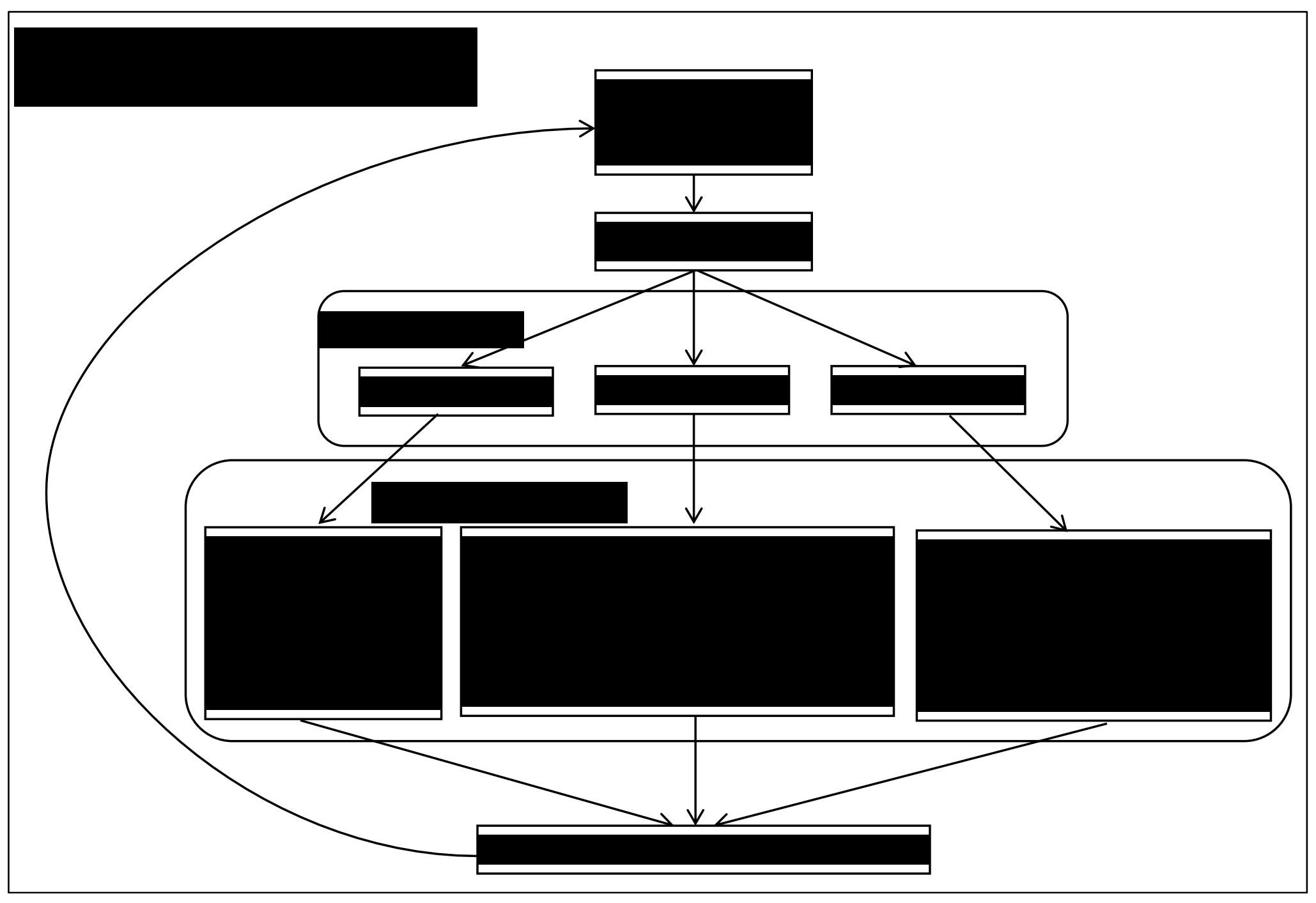

 
In developing the codes, I found it easiest to work line-by-line simultaneously with each of the three versions of the text, as the synoptic alignment facilitated recognition and identification of traits that corresponded among the versions. For example, in line $3 \mathrm{i}$ of the text (cf. Appendix B), I assigned the code $I K A B 9$ to the verbs in all three versions: "staggered" (Zitkala Ša); "walked" (Deloria); and "ran” (Walker), as all indicate the same action taken by the character Iktomi, which is to move past the Ducks. I did not have to distinguish between the versions at the coding level due to the tabular format of the alignment; later, since each version was lined out in separate sections of the table, I was able to easily tally the occurrences separately as specific to each version.

Using these clearly defined classifications of traits, subcategories, and categories, I analyzed the coded text to discern patterns that arose within each individual version of the story as well as patterned relationships that seemed to carry across the versions. My analysis focused on the aspects of character, first with regard to individual character figures, and then I made comparisons between characters, again looking for patterns and relationships that create avenues for conveying information of cultural significance. By comparing characters against other characters within the same story, I was able to isolate their unique characteristics and derive information about the relationships between them. Then, comparing and contrasting aspects of character across the three different versions, I was able to discern consistent or deviating patterns in how the storytellers portray and evolve the characters and storyline. Some anticipated themes became apparent relating to 
the use of symbolism, metaphor, physical and iconic aspects of the characters, and other facets relevant to tribal philosophy, which I return to in Chapter 4.

I conclude my discussion of the first phase of the data coding procedure by reaffirming the novel significance of my approach. My data coding development process involved numerous iterations of identifying character traits and grouping/regrouping them into classifications based on quality and type. I had to create this system because no precedent exists for this type of detailed analysis of narrative text. The painstaking, diligent effort I applied to systematically organize the text data in the manner I have described and implemented has resulted in a robust data coding method that holds integrity on account of its logical argumentation. The method is thorough and complete; furthermore, in view of the fact that I have used it here in my analysis of Iktomi, I have demonstrated that it works.

I now turn to the component of the methodology where I describe the process I used to discern more nuanced information regarding character relationships through the derivation of polarity-code correspondences.

\section{Phase 2: Polarity-code correspondences.}

A second approach to characterization involves assessing the coded data according to what I term "polarity-code correspondences", which pairs the traits according to their opposing qualities. With character identification and summarization of the data codes completed, I used this method to identify, reaffirm, and clarify relationships between distinct characters and between the characters and their environment. 
The traits sorted into the polarity-code groups provided the means to further ascertain the basic nature of the characters as the different narrators portrayed them while the polar oppositions organized the characters into types by nature and inclination and established more clarity about their standing in relationship to one another. The similarities and differences among the characters as gleaned from the polarity-code arrangements served to provide insight into how the characters relate, interact, and support each other's role in the story.

I regrouped the character traits that I condensed into the 15 subcategories according to their type coding into five sets of polarities or oppositions: (1) NonhumanHuman; (2) Conscious-Unconscious; (3) Group-Individual (referring to focus or orientation); (4) Intentional/willful-Passive/receptive; and (5) Named-Not named.

I intended that all of the polarity-code groups would bring to light subtleties about the characters that would enable me to ascertain their respective roles, motivations, and import to the story, including any possible cultural value or message that may be encoded in their representation and activities.

\section{Derivation of Paired Polarity-Code Correspondences.}

To arrive at these polar sets of traits, I scanned for patterns in the relationships between the types of traits among the 15 character traits codes and made a judgment about each one's suggestive effect on the determination of the correspondence to a polarity pair. I created a Polarity-Code/Character-Code Correspondences Template (cf. Appendix D), which I used as a guide to assess the traits for each individual character in order to control and maintain uniformity in arriving at correspondences for the characters. 
I outline my rationale for determining associations between character traits codes and each paired polarity-code grouping in the following subsections.

\section{NH:Nonhuman-HU:Human.}

Various characters exhibit traits representative of human or nonhuman entities or both. Offering a sense of how the narrator perceived the character, this distinction shed light on the character's role and importance in the tale as well as insight into how the audience might relate to specific characters, which may indicate particular cultural values imbued in the message. I sorted character traits into these groups based on whether they could pertain to a human entity versus an animal, plant, mineral, or elemental (i.e., nonhuman entity). In fact, I linked all traits except for those coded Rpl:Nonhuman in the HU:Human polarity group since they can be exhibited in some manner by a human being. Conversely, I excluded a few of the character feature codes from the NH:Nonhuman ${ }^{8}$ group because they require a certain capacity for reasoned action or intent capable only by humans. Excluded traits include Rp2:Human, Rp6:Powerlessness, Ab7:Skills, Ab10:Willful Intent, At12:Disposition, At13:Behavior/attitude-negative, At14:Behavior/attitude-positive, and At15:Egocentrism.

CO:Conscious-UC:Unconscious.

The polarity between conscious and unconscious acts and motivations of the characters also highlighted their specific roles and importance to the story and revealed clues about characters' relationships to one another. Examples of traits I included in the CO:Conscious group are those coded Rp5:Recognition and Ab7:Skills, among others. A

\footnotetext{
${ }^{8}$ I caution the reader not to confuse the polarity-code groups NH:Nonhuman and HU:Human with the character traits codes Rp1:Nonhuman and Rp2:Human.
} 
character that is personally acknowledged by name indicates an entity that possesses awareness of self, or at least is so recognized by another; hence, the character is a conscious being. The ability to learn skills also requires awareness and reasoning ability, and would, therefore, also indicate a conscious being. As can be seen in the PolarityCode template, I included several character traits in the correspondence with consciousness based on these criteria, including Rp6:Powerlessness, Ab8:Sensory/rational/emotive, Ab10:Willful Intent, At12:Disposition, At13:Behavior/attitude-negative, At14:Behavior/attitude-positive, and At15:Egocentrism. I linked only one subcategory of traits with the $U N$ :Unconsciousness groupAt1:Appearance/personality/inclination. I determined this set of traits to be wholly unconscious, since an entity has no control over these innate characteristics of being. Although one might argue that other specific character traits should also be included as unconscious polarity traits, in my rationale, I considered that the polarity groups have less to do with individual traits but are defined by the entire set of traits that I grouped into each one of the 15 character codes. For example, the character trait Ab8:Sensory/rational/emotive might suggest unconsciousness in the sense that perceptive senses are involuntary; however, the subcategory also includes traits of conscious, active, emotive abilities such as speaking or singing, so I included this inclusive set of traits in the CO:Consciousness group.

GP:Group Focus-IN:Individual Focus.

Character traits may also be sorted based on whether they indicate a focus on GP:Group orientation or IN:Individual self-interest. Traits that point to group focus 
include Rp4:Pronoun-Other and Rp5:Recognition, since both of these subcategories reflect acknowledgement of a character by beings other than the character itself.

Several character codes from each of the three categories representation, abilities, and attributes are linked to IN:Individual focus since the traits included in those codes point to the personal and individualized aspect, self-interest, or self-indulgence of the referenced entity. I included the codes Rp3:Pronoun-male, Rp4:Pronoun-other, Rp5:Recognition, Rp6:Powerlessness, Ab7:Skills, and Ab8:Sensory/rational/emotive in this polarity group.

\section{IT:Intentional/Willful-PA:Passive/Receptive.}

Traits linked with the IT:Intentional/Willful polarity group relate somewhat to the conscious aspect of the character and indicate thought-out directed action or volition. Such traits include learned abilities and skills, expression, movement, reasoning, and purposeful behavior. The codes Ab7:Skills, Ab8:Sensory/rational/emotive, Ab9:Locomotion, and Ab10:Willful Intent associate with this group.

Traits corresponding to PA:Passive/Receptive polarity relate to involuntary acts or influences and victimization. Uninitiated modes of expression, such as crying, and spontaneous responses such as fright or anger also fall into this polarity grouping. The associated codes are Rp6:Powerlessness and Ab8:Sensory/rational/emotive.

\section{NM:Named-NN:Not Named.}

These two polarity groups extend the degree of distinction between the human and nonhuman nature of the characters. NM:Named corresponds to the somewhat obvious character code Rp2:Human indicating humanness of the character since humans 
bear personal names or kinship terms. However, NM:Named also corresponds to the Rp1:Nonhuman character code because of the possibility that nonhuman entities, such as animals or inanimate objects (e.g., Teddy Bear) may acquire personal name recognition. Indeed, in Zitkala Ša's version of the Iktomi story, one of the Ducks is named "Skiská" and Iktomi addresses the Wolf/(ves) with the proper "Gray Wolves." The character code Rp5:Recognition also corresponds to this polarity since personal acknowledgement of a character, as described in the Categorization of traits section above, strongly ascribes qualities of rationality, consciousness, and logical reasoning acumen to the character and suggests the possibility of communication and reciprocation. For example, in both Deloria's and Walker's versions, Iktomi addresses the Tree(s) by the kinship term "Little Brothers" with the expectation that they will respond.

The NN:Not Named polarity group corresponds only to traits coded Rp1:Nonhuman since, by the definition given earlier, such references indicate beings or entities that are decidedly not human in nature.

\section{Illustration of Polarity-Code Determination.}

The following example illustrates how the character codes relate to the corresponding polarity groups. As presented in the Polarity-Code/Character-Code Correspondences Template in Appendix D, I listed the character codes and their generic descriptors (cf. Appendix C) in the first two columns and labeled the polarity-code groups across the top row. The "x" marks in the boxes in the columns below the polarity codes establish their link to the individual character codes which I determined based on my intuitive sense of the likelihood that an affective relationship exists between the two 
types of codes. For example, the data code Ab7:Skills from the abilities category

indicates a skill (e.g., to use tools) and I have generically associated Ab7:Skills with the following polarity-code correspondences:

$\sim$ HU:Human (in contrast with NH:Nonhuman), since the ability to use tools is essentially a human characteristic and employs reasoning;

$\sim$ CO:Conscious, in the sense that the use of tools is a learned skill requiring awareness and conscious thought;

IN:Individual Focus, because skill acquisition is a personal undertaking which cannot rely on others to do the learning;

IT:Intentional/Willful, since the learning of a skill requires direction of a desire to effect an outcome.

Likewise, I have associated the attribute code At15:Egocentrism with the same four groups of polarity-codes for similar reasons, with the added consideration that the combined quality of attention directed toward self and desired outcome for one's own benefit is also embedded in the code. I made similar general associations for each of the remaining character codes using the same process of reasoning.

I found it necessary to note the actual instances of character traits as they function in the texts in order to arrive at the correspondences particular to each unique character. The significance of the polarity codes proved meaningful only through the character traits as they came to light in the text through the coding and analysis processes by reason that not all subcategories of traits achieved representation for every character. Since the polarity-code correspondences are directly linked to the data codes, each data code associates with one or more of the polarity codes while at the same time serving as a defining quality of the individual polarity codes. 
Character codes not only varied according to the traits exhibited by individual characters; as it may have been noticed, they also corresponded to contradicting polarity groups. For instance, the ability of Ab9:Locomotion can refer simultaneously to human and nonhuman entities since both humans and animals can move under their own power. In the case of the figure Iktomi, the character can run, walk, and climb trees, which are capabilities of humans as well as animals; therefore, in the table, Ab9:Locomotion corresponds to both NH:Nonhuman and HU:Human polarity-code groups. However, the trait may not correspond to all the possible polarity codes at the character trait level. For example, like Iktomi, the Ducks exhibit the ability to walk, which is a capability of both humans (HU:Human) and animals (NH:Nonhuman); on the other hand, their ability to fly can only be associated with nonhuman (NH:Nonhuman) entities that can move (i.e., animals or birds) since humans do not have the natural ability to fly. Hence, the character code Ab9:Locomotion in this case fits only with the polarity code NH:Nonhuman.

The relevance of these differing correspondences became more evident when I tallied and assigned the individual character codes to their respective polarity-code groups. Once I tabulated the totals, the frequency of occurrence of the traits corresponding to specific polarity codes revealed patterns that further elucidated the relationships among the characters.

As just demonstrated, I associated polarity-code correspondences with one or more data codes, recorded the number of occurrences of each character code as it corresponded to each associated polarity-code, and analyzed the data for each character individually. Then, using the template in Appendix D, I created a unique matrix 
corresponding to the specific traits relevant for each character, recording the number of occurrences of each character traits code in the appropriate column intersecting its corresponding polarities. These charts specific to each character enabled me to assess each of the identified characters in like manner according to the same sets of polaritycode correspondences.

\section{Phase 3: Pronoun usage.}

In a third approach to characterization, I assessed the personal pronouns used by the different narrators to reference the characters in their respective versions of the story. Pronoun use analysis revealed specific information about the nature and quality of the characters' self-perception as well as to how characters relate to one another. However, consideration of the pronouns in the English texts required at least some examination of enclitic use in the Dakota version. I describe my methodology for making pronounenclitic comparison in the following sections.

\section{Enclitics.}

Prior to tabulating and analyzing occurrences of pronouns in the texts, I found it necessary to first examine enclitic use in the same context in Deloria's Dakota version to ensure the viability of considering the pronouns used in the English versions of Iktomi. Because Dakota is a pro-drop language, comparing the enclitics with pronouns would allow me to ascertain that the indicators of sex between the Dakota original and the English translations are consistently represented in the texts.

First, referring to information provided by the New Lakota Dictionary (Ullrich \& LLC, 2011), I analyzed the Dakota version of the text to identify each instance of use of 
an enclitic that could denote sex of the speaker and/or the addressee. I found occurrences of the following enclitics:
$\sim$ lo, yelo, welo
assertion spoken by a man;
$\sim$ huwo
interrogative used by men in formal speech;
$\sim p o$
imperative spoken by a man to more than one person;
$\sim$ ye
mild or polite request or entreaty spoken by men and women; ye changes to we following a word that ends with $o, u, u \eta$;
$\sim$ we assertion used primarily by women and occasionally by men (pp. 820-821).

Next, I looked for instances of English pronouns used in the same context and compared them with the Dakota context to see if any discrepancies might be found. The results of this examination are delineated in the Pronoun Analysis section in Chapter 4.

\section{Pronouns.}

To ascertain information about the sex of the characters and insight into how they position themselves and relate to one another, I assessed the narrators' use of English pronouns to reference each of the characters. Using the English language versions of the texts, I considered all occurring personal pronouns in the analysis, including general referencing pronouns and numeric determiners and quantifiers (i.e., one, two, anyone) that referred to one of the characters. I did not include any general references that did not point to a specific character, such as those referring to uncharacterized props or making nonspecific reference to something (e.g., there is someone).

I used simple word counts of occurrences of specific pronouns to analyze the pronouns in two groups: (1) third person singular masculine and (2) all others in one 
group. Targeting the third person pronouns may seem arbitrary; however, I singled out the third person singular masculine pronouns because of the consistent assumption evidenced in all three texts that the title character Iktomi is male. Also, an examination of how Iktomi is pronominally referenced throughout the tale provided additional insight into his role in the story as well as his relationships to the other characters.

For example, the reference of he or him attributes a quality of individual personal identity to the character as opposed to the impersonal it or collective they; individual personal identity indicates a role of significance to the plot and can help orient the referenced character in relationship to the others as the plot unfolds. Furthermore, analysis of pronoun use offered insight into the narrator's own intention and purpose for telling the story by the way she or he represented and attributed qualities of individuality to the characters.

To code the data for pronoun usage, I worked through the texts and marked each instance of the pronouns according to their type: I assigned the code Rp3 (Pronoun-male) for third person singular masculine references, case inclusive; and Rp4 (Pronoun-other) for all other pronouns with regard to person, number, and case as a single group. Next, I determined which character each pronoun references and added the character identifier to the pronoun code. Thus, to illustrate with example codes, in an instance where the pronoun he references Iktomi, I gave the trait the code IKRp3. For an instance of the pronoun it, where the pronoun refers back to the Wolf, I assigned the trait the code WORp4. Upon completion of the data coding of pronoun use, I tabulated and analyzed the results, which I present in Chapter 4. 


\section{Documenter Motivation}

Documenter motivation also presented an important consideration with regard to understanding character portrayal in the Iktomi texts. The particular conditions surrounding each of the informants and participants in the storytelling process, whether veiled or overt, may have influenced or affected aspects in the outcome of the story versions. Such factors could suggest a basis of rationale for incorporating certain details, nuances, omissions, and other subtleties into the narration. Along with the documenters themselves, I examined the people who came into contact with them as mentors, associates, contemporaries, and informants and who provided insight into the documenters' motivations for writing and recording their respective texts. By observing the historical context in which the participants emerged and engaged one another, the social and political issues they faced, and the personal obstacles they had to overcome, I was able to establish a possible link to the story and the ways it was told for different audiences in different circumstances. Based on the background information given in Chapter 2, I reexamined the circumstances surrounding the participants, particularly the documenters Zitkala S̆a, Deloria, and Walker, against the backdrop of the themes and patterns emerging from the text data to ascertain how their role in the storytelling process may have contributed to the form rendered. Understanding affective influences on those who listened and documented along with the documenters' relationship to the data helped contextualize the story as it was rendered. Such contextualization afforded an opportunity to more perceptively grasp the cultural import of the story and of the genre of the Dakota myth narrative. (At the same time, I am aware that I cannot inject my own 
perceptions into the personal motivations of the individuals involved but only as they themselves have expressed their personal experiences, feelings, and perceptions.)

\section{Calculation of Measures}

In this section, I outline the procedures I followed to quantify the data for analysis; later in Chapter 4, I discuss the results of the calculations I applied to compare the character traits and polarity-codes and their various combinations.

I used the Find function in the Microsoft Word 2007 application software to obtain simple word counts of character traits codes and spreadsheets in Microsoft Excel 2007 to compile the data and perform simple calculations. First, I tallied the occurrences of the individual codes, arriving at subtotals for traits specific to each character and according to each version and arranged them in tabular format. Then, I looked for patterns of distribution and relationship with regard to describing the individual characters and the relationships between characters in each version.

\section{Character traits codes: Simple word counts of occurrences.}

In the first phase of data coding process, I compiled the character traits code data according to one, the number of individual code occurrences by character and version and two, individual code occurrences per character compared with the total of the same code for all characters. These measures facilitated my assessment of the characters' range and depth of characterization from different perspectives.

As indicated earlier in the methodology, the first step in this phase involved sorting the character traits into the 15 data codes in the three categories representation, abilities, and attributes. I then summed the number of occurrences of each code 
according to specific character and version. The number of code occurrences provided a snapshot of which types of traits the characters exhibit and gave me the information needed to define and describe the characters based on the types of traits that make up the code.

Character traits code comparisons revealed contrasts between the characters that helped with discerning their similarities, differences, relationships, and relative stance. Numbers of code occurrences compared among the versions enabled a glimpse of how character portrayal contrasted among the different versions through the emphasis and focus the particular narrator placed on the descriptive traits they used in developing their characters. These numbers provide a sense of how the individual codes weigh against one another as well as how in composite they project the overall image of each character while indirectly aiding with discerning the relationships between characters in the different versions.

For the purpose of illustration, Table 1 presents a sample of the data specific to the character Iktomi which indicates the number of occurrences of each character traits code found in the three different versions, herein referred to as $\mathrm{Z}, \mathrm{D}$, and $\mathrm{W}$ representing Zikala S̆a, Deloria, and Walker, respectively.

To arrive at the values in the table, I first counted the number of instances of the codes for each individual character for the three versions. Referring to the 15 different codes corresponding to the character traits, I performed a search on each unique code one at a time using the Find function in the Home tab in Microsoft Word 2007 and inserted the number of matches into an Excel spreadsheet for subsequent calculation. 
Table 1.

Number of Occurrences of Traits Codes for Character Iktomi

\begin{tabular}{|c|c|c|c|c|c|c|c|c|c|c|c|c|c|c|c|c|}
\hline & \multicolumn{16}{|c|}{ Character Traits Codes } \\
\hline Version & 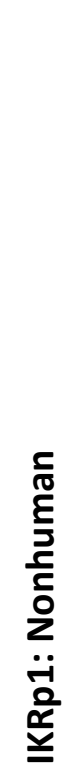 & 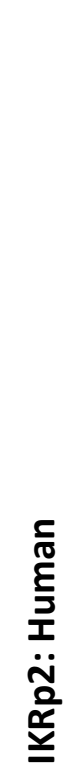 & 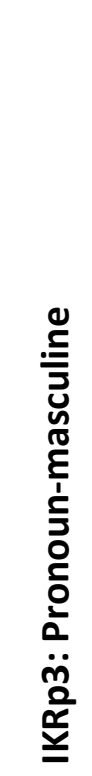 & 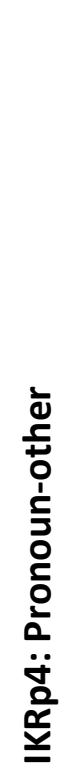 & 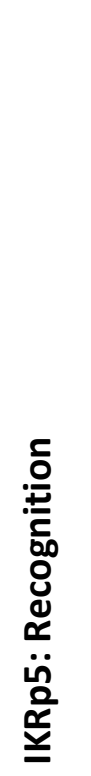 & 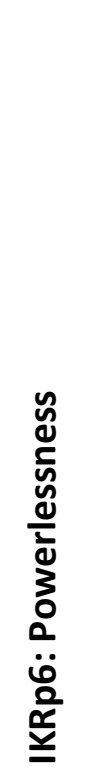 & 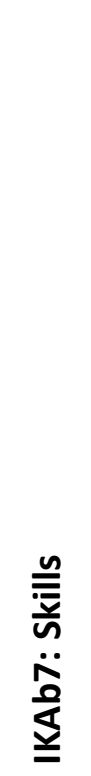 & 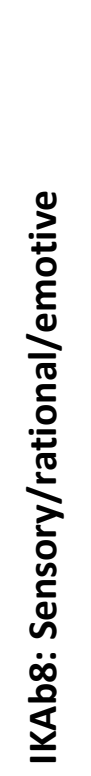 & 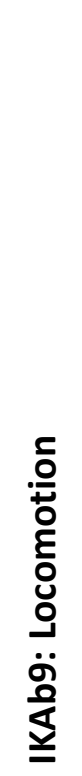 & 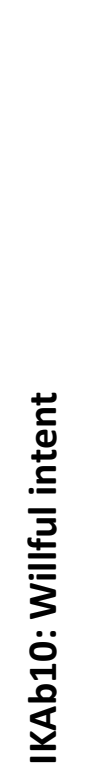 & 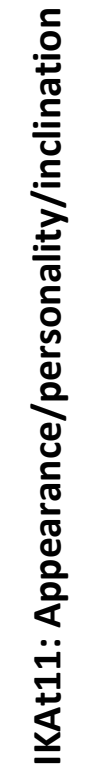 & 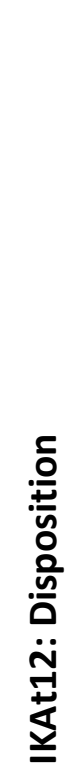 & 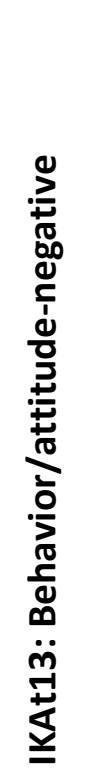 & 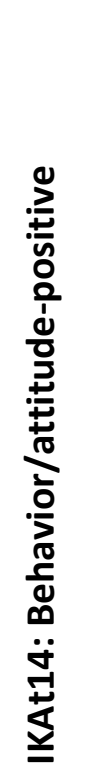 & 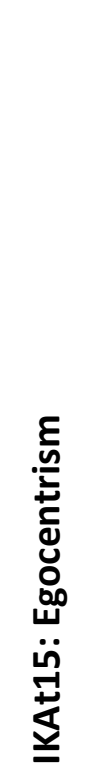 & $\begin{array}{l}\overline{\widetilde{0}} \\
\stackrel{0}{\circ}\end{array}$ \\
\hline Zitkala S̆a & 43 & 37 & 112 & 25 & 3 & 5 & 13 & 30 & 7 & 5 & 13 & 18 & 13 & 2 & 7 & 333 \\
\hline Deloria & 13 & 6 & 58 & 22 & 4 & 2 & 13 & 19 & 12 & 10 & 1 & 6 & 13 & 4 & 4 & 187 \\
\hline Walker & 24 & 8 & 90 & 22 & 2 & 2 & 18 & 32 & 15 & 10 & 2 & 4 & 15 & 2 & 11 & 257 \\
\hline Total & 80 & 51 & 260 & 69 & 9 & 9 & 44 & 81 & 34 & 25 & 16 & 28 & 41 & 8 & 22 & 777 \\
\hline
\end{tabular}


As an example, for Iktomi, the total number of instances for the code IKRp1 resulted in 43, 13, and 24 for Z, D, and W, respectively, totaling 80 for all three versions. After I tabulated all the code counts, I added the numbers together across the rows and down the columns for all the different codes for the character, which, for Iktomi, resulted in 333,187 , and 257 for $\mathrm{Z}, \mathrm{D}$, and W, respectively, and 777 totaled for all three versions combined. I repeated the process for each of the remaining six potential characters to arrive at respective summed totals of all the character traits codes pertaining to each one.

In Step 2 of this phase of the data coding process, I arranged the data to compare the total of individual codes pertaining to each character with the total of the same code for all characters. First, I added all the occurrences for a single code exhibited by each character in each version and inserted the values in a spreadsheet. Then, I calculated the sums across the rows and down the columns to obtain totals of the single code for each character and version, and then a total of all characters and versions combined.

To illustrate, Table 2 details the number of instances of the single code $R p 1$ for each of the seven potential characters occurring in each version along with the combined total for all characters. The Rp1 word counts for Zitkala S̆a's version amounted to 43 for Iktomi, 27 for the Ducks, 2 for Skiská, and so forth. Having obtained all the individual word counts, I next totaled the counts for all the characters in each version, resulting in 106,45 , and 68 codes for the versions $Z, D$, and $W$, respectively. Then, I repeated the process to obtain code totals for all 15 character traits codes pertaining to each character in the three different versions. 
Table 2.

Occurrences of Character Traits Code Rp1 for All Characters and Versions

\begin{tabular}{|c|c|c|c|c|c|c|c|c|}
\hline \multirow[b]{4}{*}{ Version } & \multicolumn{7}{|c|}{ Characters } & \multirow[b]{4}{*}{ Total } \\
\hline & Iktomi & Ducks & Skiská & Wind & Tree $(s)$ & Wolf/(ves) & Fire & \\
\hline & \multicolumn{7}{|c|}{ Occurrences of Rp1:Nonhuman Traits Codes by Character } & \\
\hline & IKRp1 & DURp1 & SKRp1 & WIRp1 & TRRp1 & WORp1 & FIRp1 & \\
\hline Zitkala $\breve{S a}$ & 43 & 27 & 2 & 5 & 11 & 9 & 9 & 106 \\
\hline Deloria & 13 & 8 & 0 & 3 & 4 & 9 & 8 & 45 \\
\hline Walker & 24 & 7 & 0 & 3 & 4 & 21 & 9 & 68 \\
\hline Total & 80 & 42 & 2 & 11 & 19 & 39 & 26 & 219 \\
\hline
\end{tabular}

\section{Polarity-code measures.}

As I described earlier in this chapter, the traits sorted into the polarity-code groups provided further insight into the basic nature of the characters and their relationship to one another. The similarities and differences among the characters, as gleaned from the polarity-code arrangements, served to provide insight into how the characters relate, interact, and support each other's role in the story.

To analyze the polarity-code correspondences data, I started by sorting the number of occurrences of individual codes (e.g., as tabulated in Tables 1 and 2) for each character into the 10 polarity-code groupings Non-human, Human, Conscious, Unconscious, Group-oriented, Individual-oriented, Intentional-willful, Passive-receptive, Named, and Unnamed as defined earlier in the methodology. I distributed the codes in accordance with Polarity-Code/Character-Code templates devised for each of the characters, which I will describe shortly. Then, I made several calculations to obtain numerical measures for evaluating and comparing characters and versions based on their respective polarity-code correspondences. 


\section{Modified Polarity-Code/Character-Code templates for individual characters.}

To prepare the data for analysis, I created a modified polarity-

code/character-code correspondences chart specific to each of the five $\mathrm{e}^{9}$ characters, which I based on the template in Appendix D. I compared the character traits codes exhibited by the individual character with the Polarity-Code/CharacterCode Correspondence Template, however, I omitted those character traits codes that did not link to any polarity group for that particular character. In other words, each character's chart showed only the matches between actual occurrences of character traits codes that character exhibited and the polarity group or groups those codes correspond to. This process resulted in individual custom templates tailored to each of the five characters Iktomi, Ducks, Skiská, Wolf/(ves), and Tree(s). Since Iktomi exhibits all 15 character traits codes, that chart serves as the base template provided in the appendix.

As a procedural note, I could have entered the relevant numbers of traits code occurrences directly into the main template instead of creating additional characterspecific charts; however, due to the volume of data to be transferred, the extra step facilitated keeping all the numbers organized while assuring that I accounted for all relevant correspondences.

\footnotetext{
${ }^{9}$ The careful reader will have noticed that I refer to only five of the seven originally identified characters. Initially, I based character determinations on the code occurrences for all seven potential characters. Once I narrowed the characters to the five active participants in the story, further consideration of those eliminated as contenders was no longer necessary.
} 


\section{Compilation of polarity-code correspondences data.}

Next, I proceeded to compile the polarity-code correspondences data according to the newly created custom Polarity-Code/Character-Code Correspondences Templates and the tabulated character traits codes data (cf. samples in Tables 1 and 2).

To demonstrate the procedural logic for how I arrived at the polarity-code correspondences, I provide an example using sample data pertaining to the character Iktomi and the polarity code NH:Nonhuman. I incorporate sample data from Table 1 and refer to the template in Appendix D to encapsulate a condensed version of the extracted data which is shown in Table 3.

As noted in the template for Iktomi (i.e., Appendix D), the polarity-code group NH:Nonhuman corresponds to the character traits codes: IKRp1; IKRp3; IKRp4; IKRp5; IKAb8; IKAb9; and IKAt11. These codes correspond to the " $\mathrm{x}$ " marks under the column heading NH:Nonhuman in the template. I listed these codes in the first column in Table 3; then from Table 1, I copied the number of character traits codes occurrences of each of the respective codes for each version into Table 3 under the column headings Zitkala S̆a, Deloria, and Walker. Next, I totaled the columns in Table 3, arriving at 233 occurrences for Zitkala S̆a, 129 occurrences for Deloria, and 187 occurrences for Walker. 
Table 3.

Iktomi's Character Traits Codes Corresponding to Polarity Code NH:Nonhuman

\begin{tabular}{lrrrr}
\hline \multirow{2}{*}{$\begin{array}{l}\text { Character Traits Codes corresponding to } \\
\text { NH:Nonhuman for character Iktomi }\end{array}$} & \multicolumn{3}{c}{$\begin{array}{c}\text { No. Code Occurrences } \\
\text { per Version }\end{array}$} & \multirow{2}{*}{ Total } \\
\cline { 2 - 4 } & Zitkala Sa & Deloria & Walker Z,D,W \\
\hline IKRp1:Nonhuman & 43 & 13 & 24 & 80 \\
IKRp3:Pronoun-Male & 112 & 58 & 90 & 260 \\
\hline IKRp4:Pronoun-Other & 25 & 22 & 22 & 69 \\
IKRp5:Recognition & 3 & 4 & 2 & 9 \\
IKAb8:Sensory, Rational, Emotive & 30 & 19 & 32 & 81 \\
\hline IKAb9:Locomotion & 7 & 12 & 15 & 34 \\
IKAt11:Appearance/personality/inclination & 13 & 1 & 2 & 16 \\
\hline Total Occurrences for All Codes: & $\mathbf{2 3 3}$ & $\mathbf{1 2 9}$ & $\mathbf{1 8 7}$ & $\mathbf{5 4 9}$ \\
\hline
\end{tabular}

I repeated the procedure for all of the relevant polarity-code groups to complete the data matrix. Then, to obtain a full set of data charts to compare results among the characters, I performed the same procedures for all the remaining four characters, creating a data matrix of polarity-code correspondences for each one.

\section{Polarity-code calculations.}

Following derivation and charting of the polarity-code correspondences for the five characters, I made various calculations using the number of polarity-code occurrences to derive quantitative measures with which to compare characters among the different versions of the story. Initially, the overall polarity-code totals combined for the three versions provided a general overview of the characters and their relationships, broadly interpreting the sequence of events and character interactions without specific attention to the detail of the individual adaptations. Then, comparing each character's share of the total for the individual versions offered more detail about how the narrators 
characterized and positioned the characters relative to one another within their respective versions of the tale. These data indicated narrator emphasis on one or another quality in each of the paired oppositions for the characters which, in turn, provided insight into the characters' manner of being and the nature of their roles and interrelationships throughout the story.

\section{Weighted versus unweighted values.}

Two measures delved deeper into the nuances embedded in the polarity-code correspondences data: unweighted and weighted calculated values. The unweighted data show more variation among the characters and highlights their differences and distinctions in relational positioning while the weighted data tend to merge the characters' qualities into unifying themes that link the characters ideologically to one another and hint at more subtle connectors among them.

The weighted and unweighted values incorporate the numbers of polarity-code occurrences expressed in terms of relative percentages, that is, a character's percentage share of polarity-codes compared to the code totals for all the characters. For example, as indicated in Table 3, the number of codes in the polarity-code group NH:Nonhuman for Iktomi occurring in Zitkala S̆a's version adds up to 233; for the remaining characters, the totals are 118 for the Ducks, 15 for Skiská, 24 for the Tree(s), and 34 for the Wolf/(ves). ${ }^{10}$ The total for all characters combined is 424; therefore, Iktomi's share of the codes in Zitkala S̆a's version amounts to $(233 \div 424)$ x $100=55.0$ percent. For the combined three versions, the percentage share of codes in the NH:Nonhuman group for the character Iktomi derives from dividing Iktomi's total codes into the total for all

\footnotetext{
${ }^{10}$ I have not provided data tables for characters other than Iktomi.
} 
characters in all the versions: $(549 \div 1058)$ x $100=51.9$ percent. I have provided the results of these calculations pertaining to the five characters in Table 4. 
Table 4.

Unweighted Polarity-Code Correspondences Summary in Percentages

Unweighted Polarity-Code Paired Groupings of Character Traits in Percentages

\begin{tabular}{|c|c|c|c|c|c|c|c|c|c|c|}
\hline Character & $\begin{array}{l}\delta \\
\delta \\
\frac{\delta}{\delta} \\
\frac{c}{\delta} \\
\delta\end{array}$ & $\begin{array}{l}\delta \\
\delta \\
\hat{\Sigma} \\
\text { 1 }\end{array}$ & 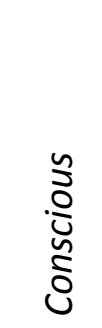 & 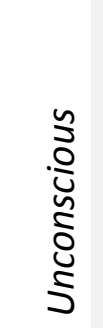 & 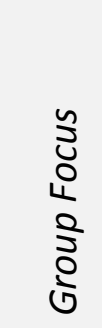 & 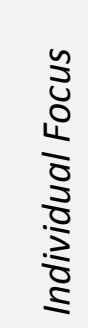 & 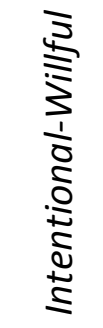 & 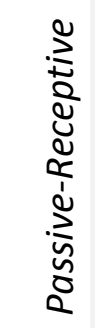 & $\begin{array}{l}\text { ¿ } \\
\frac{\delta}{\delta} \\
z\end{array}$ & 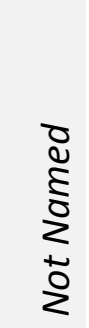 \\
\hline Iktomi & 51.9 & 57.7 & 59.9 & 14.2 & 46.2 & 65.2 & 60.0 & 62.1 & 63.6 & 44.0 \\
\hline Ducks & 24.0 & 24.0 & 15.2 & 60.3 & 24.3 & 33.6 & 16.2 & 18.2 & 0.9 & 22.8 \\
\hline Skiska & 3.0 & 3.0 & 3.7 & 3.4 & 2.4 & 6.6 & 3.8 & 4.1 & 1.4 & 1.1 \\
\hline $\operatorname{Tree}(s)$ & 6.2 & 4.0 & 3.7 & 10.3 & 12.4 & 7.1 & 2.4 & 4.1 & 13.1 & 10.3 \\
\hline Wolf/(ves) & 13.7 & 12.2 & 13.9 & 9.5 & 14.8 & 21.1 & 16.9 & 10.8 & 20.7 & 21.2 \\
\hline
\end{tabular}


The measures just discussed involved unweighted values, that is, I calculated them from the number of occurrences without adjusting them according to a relative scale. However, since the polarity-code groups comprise nonuniform combinations of the character traits codes, the values can be misleading and exaggerate the apparent influence of certain polarity-code groups on the character's representation in the story. To correct for this potential skewing of the data, I recalculated the percentages weighted against the total number of potential instances of a code type represented in the template (cf. Appendix D).

To calculate weighted percentages for the data, I first derived a total of 56 possible occurrences by adding all the points of intersection between the character traits codes and the polarity codes in the Polarity-Code/Character-Code Template. For each polarity-code group under the column headings, I subtotaled the number of types of character traits codes that comprise the group; then I added all the subtotals to arrive at the overall total of 56 .

Next, to obtain the relative weights for each polarity-code group, I divided the subtotal of character traits code types for the group into 56 and multiplied by 100 to arrive at the weighted share of the total for the polarity-code group, expressed as a percentage of the total possible. For example, Iktomi shows 7 possible code types in the polarity-code group NH:Nonhuman; hence, $(7 \div 56)$ x $100=12.5$ percent. Therefore, the group NH:Nonhuman pertaining to Iktomi represents a weight of 12.5 percent of all possible instances of the 56 codes. 
I then multiplied each character's percentage by the number of occurrences of codes in the polarity-group for each version to obtain the characters' weighted shares of coded polarity traits from the data tables. Iktomi's weighted share of the polarity-code NH:Nonhuman amounts to $(.125 \times 549)=68.6$. These calculated weighted values are displayed in Table 5. 
Table 5.

Weighted Polarity-Code Correspondences Summary

Weighted Polarity-Code Paired Groupings of Character Traits as Normed Values

\begin{tabular}{|c|c|c|c|c|c|c|c|c|c|c|}
\hline Character & $\begin{array}{l}\delta \\
\delta \\
\delta \\
\frac{\delta}{\delta} \\
\delta \\
z\end{array}$ & 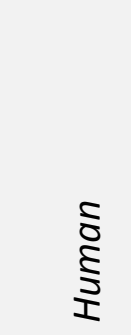 & 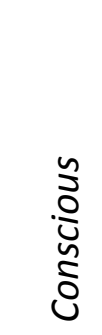 & 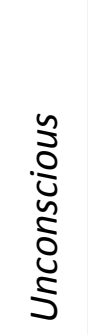 & 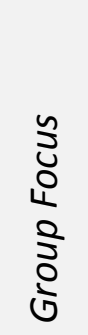 & 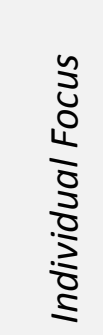 & 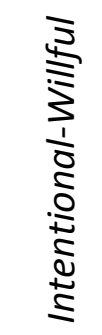 & 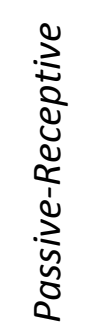 & $\begin{array}{l}\text { d } \\
\text { है } \\
\dot{z}\end{array}$ & 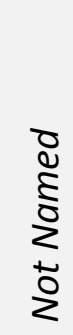 \\
\hline Iktomi & 68.6 & 174.3 & 33.4 & 0.3 & 2.8 & 90.0 & 37.9 & 3.2 & 7.5 & 1.4 \\
\hline Ducks & 32.9 & 71.0 & 9.3 & 1.3 & 1.5 & 23.6 & 10.4 & 1.0 & 0.1 & 0.8 \\
\hline Skiska & 4.0 & 9.0 & 2.3 & 0.1 & 0.1 & 4.7 & 2.4 & 0.2 & 0.2 & 0.0 \\
\hline Tree(s) & 8.3 & 11.8 & 2.3 & 0.2 & 0.8 & 5.0 & 1.6 & 0.2 & 1.6 & 0.3 \\
\hline Wolf/(ves) & 18.5 & 36.0 & 8.5 & 0.2 & 0.9 & 14.8 & 10.9 & 0.6 & 2.5 & 0.7 \\
\hline
\end{tabular}




\section{Summary of Methodology}

Throughout Chapter 3, I have described the procedures and measures I used to quantify and analyze the text data derived from the Iktomi story. In

summary, first, I provided the cultural context for the story under examination and described how I selected the three versions for the study along with my analytical approach to the data. My description encompassed synoptic alignment of the texts, character identification, devising a system to code the data, and detailed coding of the character traits in three phases: character traits; polarity-code correspondences; and pronoun usage. As part of the discussion, I explained the function of different types of character traits that led to my organization of the data codes. Next, I described how I derived polarity-code correspondences and reorganized the character trait codes into groups defined by them in order to assess the data from the perspective of paired contrasts. Following that discussion, I addressed the use of pronouns and enclitics in the texts and presented a brief overview of how the Dakota system of enclitics came into play in determining the sex of the characters in the Dakota version of the story. I then showed the correspondence of meaning between the enclitics and their English pronoun counterparts in the translated versions. Next, I described how I derived codes which pertained to English pronoun use in the three texts.

After discussing the procedures I laid out for character trait analysis, I addressed how I approached an assessment of the potential influence the documenters' personal experience and background may have contributed to the 
rendering of their respective texts. In the final section of this chapter, I presented detailed procedures I employed to calculate measures to quantify the data and give meaning to it. These procedures included compilation of code tallies for each character in each version via simple word counts and then through their polarity-code correspondences while taking into consideration normalized values based on the relative occurrences of specific codes relating to specific characters.

In Chapter 4, I present the results of my analysis of the data, followed by my interpretation and insights in Chapter 5 of what I gleaned from the analysis that support my thesis that this Iktomi story represents a prototype for the Dakota vision quest ritual. 


\section{Chapter 4: Data Results and Analysis}

In this chapter, I present the results of data analysis, examine them as they relate to the processes outlined in the methodology, and offer my understanding of what the data revealed in reference to those processes.

In analyzing the data, I begin with the synoptic alignment of the texts which confirms the versions as variations of the same story. Next, I discuss the information gleaned from the character traits codes in terms of how their distribution assists with defining and identifying the characters and their roles and relationships and how the characters compare among the different versions. I will discuss the three measures I used to approach the data which include consideration of the number of code occurrences, the regrouping of the codes into paired polarity-code oppositions, and the frequency of use of English pronouns in the translated versions. Finally, I discuss the effects and influence that the context of the story's telling may have had on the versions of the three texts in the form used in this study. I will show how the story and its characters may have taken shape based in part because of the sex and experiential background of the individual narrators, translators, documenters, and audience, i.e., the story's external participants.

In synopsis, my analysis of the data revealed aspects of the story that affirm the following key points which support my thesis:

$\sim$ The story features five characters, all of which represent aspects of humanness;

All five characters are portrayed simultaneously as human and nonhuman, e.g., plant or animal;

$\sim$ Iktomi stands as the primary character and protagonist of the story; 
$\sim$ The Ducks as a group represent the feminine aspects of characteristic nature in human audience members;

$\sim$ The Wolf/(ves) represent the masculine aspects of characteristic nature in human audience members;

$\sim$ Skiská and the lead Wolf serve as mouthpieces for their respective groups and as individuals that are singled out with which audience members can more readily identify;

$\sim$ The Tree(s) symbolize the duality in human nature and act as a connecting link between the heavens and the earth, that is, the spiritual and material/physical dimensions of existence. The Tree(s) represent the crossroads of choice and the decision facing the seeker regarding which course of action or direction to take in life.

\section{Synoptic Alignment}

Synoptic alignment of the texts revealed essential continuity and consistencies across the texts. First, in accordance with the description of episodic story components introduced in Chapter 2, the three versions of Iktomi adhere to a uniform outline of plot and storyline, broken into four cyclic episodes as charted in Appendix A. I first estimated the break points between consecutive episodes, predicting at first that they would coincide with distinct activities of the most prominent character Iktomi. The other characters, indeed, only came to notice through Iktomi, and the episodic cycles revolved around Iktomi's activities. Each episode comprises a discrete encounter between the protagonist Iktomi and one of the other primary or secondary characters in which an activity is initiated, followed through, and completed. Stated within the frameworks posited by Labov, Bierhorst, and Georgakopoulou (cf. Chapter 2), each episode internally encompasses the cycle of orientation, incident, crisis, result-consequence, and return to the starting point. I found no overlapping interactions between Iktomi and other 
characters; therefore, since Iktomi completes his interaction with each character exclusively without engaging the next, I deemed it appropriate to draw the episodic boundaries between the distinct encounters.

The four episodes comprising the Iktomi story include Iktomi's encounters with (1) the Ducks, (2) the Tree(s), (3) the Wolf/(ves), and (4) the Wolf/(ves) again. Iktomi's movement from one location to the next or from one state of contemplation to a new one marks the boundaries between consecutive episodes. Comparing the chart in Appendix A with the synoptic text alignment as sampled in Appendix B, I determined that the episode boundaries correspond to the text as follows: Episode 1 comprises lines 1 through $14 \mathrm{~d}$ of the texts; Episode 2 comprises lines 14e through 19c; Episode 3 includes lines 19d through $22 \mathrm{~h}$; and Episode 4 runs through lines $22 \mathrm{i}$ to $27 \mathrm{e}$, inclusive. ${ }^{11}$

Iktomi's encounter with the Tree(s) may appear to be an exception to the boundary patterning since Iktomi encounters the Wolf/(ves) for the first time while still caught in the tree, and he does not find release until the Wolf/(ves) have moved on. However, if we consider the episode as involving Iktomi's wrestling with inner conflict, then, by the time the Wolf/(ves) approach him, Iktomi's struggle to break free has already finalized in surrender to his helpless predicament and subsequent reference to the Tree(s) occurs simply with the mention of Iktomi's release and excludes further interactive engagement between him and the Tree(s).

With regard to textual presentation (i.e., line and section breaks), it may be noted from the synoptic alignment that, although Deloria's system of breaking out the text into lines marks separation between complete sentences (both, in the English and Dakota

\footnotetext{
11 The text sample provided in Appendix B shows only the text lines 1 through 6.
} 
language versions), I did not find the points of separation to necessarily correspond to episodic boundaries, or even to smaller contained constituents within the episodes. Nor do the breaks occur in the same places consistently in the different versions, although the events in the storyline do cohere contextually and sequentially from one version to the next. I found only minor deviations in the order of details within the events themselves that do not impact the integrity of the storyline; hence, I assert with confidence that the three versions are one and the same tale and coincide in episodic format.

That said, I concede to certain discrepancies in the texts. As noted in Chapter 2, the text in Zitkala S̆a's version encompasses only three episodes, while Deloria's and Walker's versions include a fourth subsequent episode. Additionally, Zitkala S̆a added an introduction to her version which is not included in Deloria's or Walker's versions.

The inclusion of these additional sections of text (i.e., Introduction; Episode 4), which are not consistently part of all three texts, may result in a slight skewing of the data results in terms of frequency of word counts of certain character trait codes since additional occurrences of specific codes will be present in those sections and will not pertain to every version. For example, I recorded 10 additional occurrences of the code IKRp1 in Zitkala S̆'s introduction, and 3 and 11 more occurrences in Episode 4 of Deloria's and Walker's versions, respectively. The number of occurrences similarly increases for several more character trait codes when taking the extra sections into account, although the affected codes involve only the characters Iktomi and the Wolf/(ves). This discrepancy disallows direct comparison of otherwise comparable samples of text; however, I do not perceive this aberration to be a significant detraction 
from the essential interpretation of the text, though excluding the word counts extracted from these add-on sections from the data would ensure comparison between exact parallel versions. In defense of retaining these occurrences, I suggest the resulting effect of the skewed data is likely to manifest only in terms of further defining the two characters involved and the relationship between them, rather than in their significance in terms of character role. Furthermore, I have retained these additional occurrences in the tabulated data for two specific reasons: first, I employed the coded traits included in these sections in my process of defining the 15 character trait codes, using them to derive added details that helped me determine the nature of the characters and develop the codes that I would be using to analyze their traits; second, I used complete parallel versions of the stories in the form they were rendered in the English language, excepting the cutoff I made of Walker's version at the conclusion of the episode comprising Iktomi's involvement with the Wolf (cf. Appendix A: Episode 4). As I explained in Chapter 2, I determined the cutoff based on the premise that the added segments in Walker's version are additional cyclic episodes which do not correspond to any material in either of the other two versions. I further argue in favor of including Zitkala S̆a's introduction from the standpoint that it simply provides us additional information to elaborate on the description of a character already defined though it has no impact on the character's interplay with any other character at that point.

\section{Data Coding Results and Analysis}

I conducted the data analysis in three phases: first, I coded the text data for character traits which aided in defining the characters and their roles and 
relationships; second, I resorted the character traits codes into paired polaritycode oppositions to determine correspondences among the character traits codes which provided information to further clarify the characters' relative positioning to one another; third, I assessed the narrators' incorporation of personal pronouns in reference to the characters. The pronouns served to establish the characters' sex and reinforce the presumption of male-focus of the story in addition to clarifying relative stance among the characters.

\section{Phase 1: - Character traits codes and character portrayal.}

Arrangements of the data in terms of (1) number of individual code occurrences by character and version, (2) individual code occurrences per character compared with the total of the same code for all characters, and (3) individual code occurrences compared with the total occurrences for all codes provided a variety of perspectives for observing emergent patterns among the distribution of codes.

First, the number of individual code occurrences obtained from simple word counts provided an overview of the frequency and distribution of the 15 codes exhibited by the individual characters. This information allowed me to discern the defining qualities for determining the viability of character status for each of the potential characters. The number of total occurrences by character and version also revealed emerging patterns of similarities and differences in the portrayal of individual characters in the different versions. The patterns enabled a view of which of the coded traits the narrators employed consistently to define and describe their characters and which codes they deemed more or less important to each character's role for imparting the story's 
message. I provided a sample of these data which I expressed as simple numbers in Table 1 in Chapter 3.

Second, the relative number of individual code occurrences per character compared with the total of the same codes for all characters permitted comparison of the characters relative to one another and offered perspective on how the different narrators viewed the role of the individual characters in relation to the other characters which, in turn, enabled me to discern the relationships between characters more clearly. I expressed these data as simple numbers as well and provided an extract in Table 2 in Chapter 3.

Third, the total number of individual code occurrences with respect to each character compared with the total occurrences for all codes lent perspective on how each code weighed against the other codes and the degree to which specific traits codes helped mold the character. I obtained this information from a synthesis of the all the data charts tabulated for each of the five characters.

In the next sections I provide the results of my analysis of these three components of the data encompassing Phase I of the results and analysis that concern character definition and portrayal.

\section{Character traits code distribution.}

To begin my analysis of the text data, I synthesized the quantitative information presented in Tables 1 and 2 in Chapter 3 into Table 6, which visually summarizes which of the codes I found to be specifically associated with each of the characters and includes 
the number of instances of the coded traits. The figures in the table represent a composite of the occurrences of coded traits totaled for all three versions of the text.

\section{Table 6.}

\section{Coded Traits Exhibited by Potential Characters in Composite for All Versions}

\begin{tabular}{|c|c|c|c|c|c|c|c|c|}
\hline \multirow{2}{*}{$\begin{array}{c}\text { Character Trait Codes by Category } \\
\text { Representation: } \\
\text { Character presentation }\end{array}$} & \multicolumn{8}{|c|}{$\begin{array}{l}\text { Characters Associated with Traits Codes: } \\
\text { Number of Code Occurrences }\end{array}$} \\
\hline & $\begin{array}{l}\overrightarrow{\bar{g}} \\
\bar{\Xi}\end{array}$ & 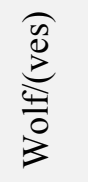 & $\begin{array}{l}\frac{n}{0} \\
\tilde{\tilde{D}}\end{array}$ & 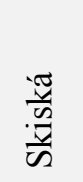 & 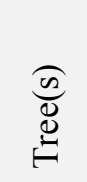 & $\frac{\pi}{3}$ & 总 & 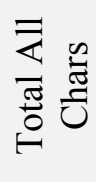 \\
\hline Rp1: Nonhuman & 80 & 39 & 42 & 2 & 19 & 11 & 26 & 219 \\
\hline Rp2: Human & 51 & 4 & 6 & ---- & 3 & --- & -ב--- & 64 \\
\hline Rp3: Pronoun: $\mathrm{ma}$ & 260 & 28 & 7 & 11 & ---- & ---- & ---- & 306 \\
\hline Rp4: Pronoun: other & 69 & 22 & 109 & 4 & 14 & ---- & ---- & 218 \\
\hline Rp5: Recognition & 9 & 3 & 2 & 1 & 7 & ---- & ---- & 22 \\
\hline Rp6: Powerlessness & 9 & ----- & ----- & ----- & ----- & ---- & ----- & $\underline{9}$ \\
\hline Subtotal Number of Cod & 478 & 96 & 166 & 18 & 43 & 11 & 26 & 838 \\
\hline Subtotal & & 5 & 5 & 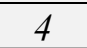 & 4 & 1 & & \\
\hline
\end{tabular}

\section{Abilities:}

Self-motivating/animating qualities

\begin{tabular}{lrrrrrrrr}
\hline $\mathrm{Ab} 7:$ Skills & 44 & 8 & 4 & ----- & ----- & 2 & ----- & 58 \\
\hline $\mathrm{Ab} 8:$ Sensory/rational/emotive & 81 & 16 & 27 & 6 & 6 & ---- & 1 & 137 \\
\hline $\mathrm{Ab} 9:$ Locomotion & 34 & 27 & 17 & 3 & 1 & 2 & 1 & 85 \\
\hline $\mathrm{Ab} 10:$ Willful intent & 25 & 7 & ---- & 8 & 4 & 4 & ----- & 48 \\
\hline Subtotal Number of Code Occurrences: & 184 & 58 & 48 & 17 & 11 & 8 & 2 & $\mathbf{3 2 8}$ \\
\hline Subtotal Number of Code Types: & 4 & 4 & 3 & 3 & 3 & 3 & 2 & 4 \\
\hline
\end{tabular}

Attributes:

Personality, behavior and character

\begin{tabular}{lrrrrrrrr}
\hline At11: Appearance, personality, inclination & 16 & 3 & 70 & 4 & 12 & 3 & ----- & 108 \\
\hline At12: Disposition & 28 & 14 & 12 & ---- & ----- & ----- & ---- & 54 \\
\hline At13: Behavior/attitude-negative & 41 & 2 & 10 & ---- & ----- & ---- & ---- & 53 \\
\hline At14: Behavior/attitude-positive & 8 & ----- & 3 & ---- & ---- & ---- & ---- & 11 \\
\hline At15: Egocentrism & 22 & 3 & ---- & ---- & ---- & ---- & ---- & 25 \\
\hline \hline Subtotal Number of Code Occurrences: & 115 & 22 & 95 & 4 & 12 & 3 & 0 & $\mathbf{2 5 1}$ \\
\hline Subtotal Number of Code Types: & 5 & 4 & 4 & 1 & 1 & 1 & 0 & $\mathbf{5}$ \\
\hline Total Number of Code Occurrences: & $\mathbf{7 7 7}$ & $\mathbf{1 7 6}$ & $\mathbf{3 0 9}$ & $\mathbf{3 9}$ & $\mathbf{6 6}$ & $\mathbf{2 2}$ & $\mathbf{2 8}$ & $\mathbf{1 4 1 7}$ \\
\hline Total Number of Code Types: & $\mathbf{1 5}$ & $\mathbf{1 3}$ & $\mathbf{1 2}$ & $\mathbf{8}$ & $\mathbf{8}$ & $\mathbf{5}$ & $\mathbf{3}$ & $\mathbf{1 5}$ \\
\hline
\end{tabular}




\section{Traits code distribution among the potential characters}

The first noticeable pattern in the data involves the distribution of coded traits and qualities the different characters exhibit in the various versions of the story. Of the seven potential characters, which I identified in Chapter 3, only Iktomi displayed occurrences of all 15 character codes. The Wolf/(ves) and Ducks also exhibited occurrences of most types of the codes, 13 and 12, respectively. Both Skiská and the Tree(s) exhibited occurrences of just over half of the different code types -8 each-while the Wind showed occurrences of 5 of the codes and the Fire exhibited occurrences of 3 codes. I will present a more detailed discussion of the distribution of codes corresponding to the potential characters shortly.

Another pattern of distribution emerges from the tabulated data as well: as the number of codes represented for the character decreases, the less descriptive the detail that is made available for the character, on the one hand, and the more impersonal the rendering of the character, on the other hand. In other words, when fewer codes occur, they tend to aggregate in the first or second category of descriptors (e.g., representation and abilities) and the narrator ascribes fewer attributes traits to the character. Traits in the representation and abilities categories provide only skeletal definitions and descriptions of the character's presentation and capabilities; however, the attributes are what give the character depth and dimension through added detail and coloring of personality traits, appearance, attitudes, and behaviors. 


\section{Defining the characters.}

To refresh our conceptualization of the signification of the 15 codes, I briefly recap them here from the descriptions I gave in Chapter 3. I occasionally refer to examples from the texts, which may be found in Appendix B.

The first group of six codes under the heading representation recognizes qualities of character presentation that signify the way others perceive the entity. The first two codes in the representation category identify the entity as distinguished between nonhuman (Rp1) and human or human-like (Rp2). The next two codes indicate anaphoric reference to the figure by other characters or the narrator through at least one use of a personal pronoun: $3^{\text {rd }}$ person singular masculine $(\mathrm{Rp} 3)$ and all other pronouns (Rp4). The fifth code (Rp5) indicates recognition of the figure as an independent living entity with its own personality and identity, expressed through personal salutation by name or kinship term by itself or others. The sixth code (Rp6) affords the character a sense of vulnerability and conscious awareness of the imbalance of power and lack of control over its own fate or destiny.

I found through the data coding five of the character candidates to exhibit traits in this representation category of data codes, excluding Wind and Fire, which exhibit only nonhuman traits and no other qualities that would suggest they possess a personal identity. Interestingly, the Tree(s) appear as both human and nonhuman, and they exact personal acknowledgment by at least one other character candidate, distinctly suggestive that they have their own personal identity. 
Codes in the second category of abilities indicate that the same five characters, along with Wind, exhibit self-motivating qualities, which is a trait of animate beings, whether human or animal. The first code in this category (Ab7) identifies the character with cognitive and motor ability to apply complex, learned skills. The next code (Ab8) associates the character with sensory, rational, or emotive ability connected to intuitive perception, reasoning of the mind, or emotional expression. The following code (Ab9) indicates locomotive ability; that is, the character is capable of moving itself from one place to another or to at least change position. The traits comprising this code infuse the characters with a sense of being alive, though again, from the Western frame of reference. The tenth code (Ab10) reveals willful intent of the character, a rational quality which requires cognitive activity and intentional motivation. The general belief that humans alone possess this quality of rationality consigns such displays in the animal world to the order of instinctual impulse.

Regarding the category as a whole, again, six of the character candidates, excluding Fire, display viable ability traits. (My exclusion of Fire's scores in the abilities category will be explained in the next segment.) Iktomi, the Wolf/(ves), and the Ducks, including Skiská, exhibit all four types of traits. Although the Ducks as a group do not exhibit willful intent (Ab10), Skiská does so as an individual member singled out from the group. Conversely, the Ducks as a group display learned skills, whereas Skiská, as an individual, does not act accordingly but does so only as a member of the collective group of Ducks. The Tree(s) and Wind also display ability traits relative to most of the codes in 
this category, reiterating the notion of individuality that the narrators may have ascribed to them.

Traits in the third category of attributes describe aspects of personality and behavior of the character candidates and help to differentiate them as conscious individuals possessing unique identities. The first two codes in this group describe natural traits innate to the character, such as appearance, personality, and inclination and (At11) individualizing behaviors and impulses of disposition (At12). According to the coded data, all of the candidates, except Fire, feature some type of innate character tendencies. The next two codes describe the character candidate in relation to the others in terms of negative (At13) or positive (At14) interaction. Only three of the potential characters —-Iktomi, the Ducks, and the Wolf/(ves) — demonstrate these traits, suggesting that only they possess an awareness of the differentiation between self and other. The final code At15 conveys personal self-awareness on the part of the character candidates, demonstrated most often as egocentrism, which only Iktomi and the Wolf/(ves) project. Skiská, the Tree(s), and the Wind display only innate traits of appearance, personality, and inclination (At11), yet no traits that would otherwise color their characters with interactive, inter-relational qualities to bring them to life as independently motivated actors. Although Skiská displays traits in the representation and abilities categories which point to status as a main player, his deficiency of attributes downgrades his role. His figure stands, instead, as an extension of his membership in the collective group of Ducks. As one of the Ducks, Skiska's apparent individual personality cannot in actuality 
be separated from the group, and apart from the others, he does not come across as a distinct entity detached from the group.

\section{Code distribution and character roles.}

The results of the code distribution yield further insight into the relative importance of the potential characters and their respective roles in the story while facilitating determination of character status. For example, starting with the least represented of the potential characters, Fire displays only three of the 15 codesRp1:Nonhuman, Ab8:Sensory/rational/emotive; and Ab9:Locomotion. While Fire is actualized in its various forms (i.e., fire, flames, ash), no other character addresses or acknowledges it, nor does it interact with any of the other characters in any of the three versions of the story. Furthermore, Fire does not display any traits of appearance, personality, attitude, or behavior. We know only, from at least one of the versions, that Fire is a nonhuman entity capable of sensory, rational, or emotive expression (Ab8) and it has the ability to move in some fashion (Ab9). However, the only two references to these two abilities occur in lines 14a and 20i (cf. Appendix B), both from Zitkala's version:

Line $\underline{\text { Code }} \quad \underline{\text { Sample text }}$

14a FIAb9: leaping flames

20i FIAb8: dead fire

Although I initially coded these traits as abilities of Fire, coding analysis revealed that these references actually serve as adjectival descriptors of appearance or state of being rather than indicate volitional acts performed by the Fire. In the end, the listener 
cannot relate to Fire in any personal sense because insufficient detail is given by any of the narrators to personalize any association with it.

Likewise, the potential character Wind exhibits similar traits related to those shown by Fire, which fall into the categories of representation and abilities. At least one narrator gives us slightly more information about Wind, imbuing it with traits suggestive of learner ability (Ab7:Skills) and conscious motivation (Ab10: willful intent) through coded traits in the abilities category. We also ascertain something about Wind's descriptive attributes of appearance or personality which tempt us to align ourselves more closely with it. However, because Wind fails to mutually interact with other characters or receive their acknowledgement, we still lack sufficient information to establish a personal relationship with the potentiality of Wind's role as a story character. Although a character's abilities help motivate its role and move its purpose through the plot, neither Wind nor Fire elicits interactive response from other potential characters.

The remaining five potential characters, Iktomi, Wolf/(ves), Ducks, Skiská, and Tree(s), all display coded traits indicating interactive relationship with other actors in the story. Four of the five are portrayed as having human characteristics; only Skiská is portrayed as solely nonhuman. However, Skiská is referenced with the personalizing masculine pronouns, suggestive of human quality; and all five potential characters are personally recognized in some manner by one or more of the other character candidates. The combination of personalizing characteristics, personal reference, and acknowledgement of the individual players strongly support the inference that they carry 
a role significant to the story plot. The added quality of humanness $(\mathrm{Rp} 2)$ exhibited by four of the players reinforces motivated purpose for their incorporation in the story.

It is difficult to distinguish character status between Skiská, the Wind, and the Tree(s) when considering that all three display a variety of similar coded traits. However, important differences factor in to set the Wind apart from the others: unlike the Wind, the Tree(s) and Skiská exhibit representational human qualities (Rp2) and sensory, rational, and emotive abilities (Ab8); other characters personally acknowledge (Rp5) the Tree(s) and Skiská, though not the Wind; the Tree(s) and Skiská display self-mobilizing and selfmotivating abilities (Ab9), while the Wind only "rushes" or "stops" without any indication of the force that drives it. Although the Wind does seem to possess certain cognitive abilities, as mentioned earlier, it fails to attain personal recognition by other characters. The Wind is also accorded traits of ability in the versions which appear to give it some of the necessary defining qualities of individuality; however, the Wind lacks any representational traits that would give it a personal identity or any attributes that might describe an intentional relationship with the other characters. Instead, what appear to be the Wind's inherent abilities turn out to be simply projections of perception and will coming through the style of narration, and its few qualities prove insufficient to ascribe it character status since it does not relate to the others in any real way. Based on these distinctions, I deduce that both the Tree(s) and Skiská must play somewhat important roles in the story, albeit not with the same significance as Iktomi, the Ducks, or the Wolf/(ves) while the Wind reduces to a nonhuman unmotivating elemental force. For these reasons and with the earlier discussion of Fire, I conclude that neither Wind nor 
Fire plays a role meriting character status. Rather, they play only peripheral noncharacter roles as elemental forces in the story.

These results would be expected, consistent with the Native perspective that the audience must be able to relate or identify personally in some way with a story's characters through their likeness, mannerisms, attitudes, feelings, and so forth. Consequently, relative to character identification, I regard the most important traits of character definition, therefore, to entail identification as a consciously motivated being; ability to learn, sense, and/or move under one's own power; personal recognition or acknowledgement by at least one other character; reciprocity between characters in the form of dialogue, interaction, or action and response; and expression of disposition, behaviors, or attitudes to evoke emotional response in the audience. Iktomi, the Ducks, Skiská, the Wolf/(ves), and the Tree(s) meet these criteria.

I have given quite a lot of space to the discussion of character definition in order to impress my conviction that the story's underlying significance resides in its characters, their roles, and their interactions, and that the character traits can provide us the detail to ascertain insight in that regard to embedded cultural information. I now continue in the next section to describe the characters' relationships.

\section{Character relationship.}

Concluding that the story involves five definite characters, having excluded Wind and Fire, I used the degree of detail provided for each character to begin to describe their relational status. I have noted that Iktomi, the Wolf/(ves), and the Ducks are represented by a majority of the 15 codes. In addition to the representation and abilities traits that 
describe the basic characters, the narrators give an abundance of information in the form of attributes that describe each of these characters in personal detail, including their personality traits, natural inclinations, thought processes, behaviors, and attitudes. In contrast, despite the recognition of Skiská and the Tree(s) as important players, the narrators provide very little additional description that would help us engage and identify with these two characters. Where we might, as listeners, emotionally involve ourselves with Iktomi, the Wolf/(ves), or Ducks from a first-person point of view, with Skiská and the Tree(s), we remain observers from a safe emotional distance. We are able to get inside the hearts and heads of the former; we can move with them, feel what they feel, and think in parallel with their musings. With the latter, we do not achieve the same sensory connection because we do not know what they think or feel. We only know from the data what they can do and how other characters perceive them. For example, Iktomi addresses the Tree(s) directly in the second person and calls them by an appropriate kinship term, while he attempts to interact with them as though they were human like himself. However, the interplay between them is rather like Quixote jousting with his windmills — real in the mind of the actor (i.e., Iktomi) though fanciful in reality. In light of these discriminating factors, I conclude that Iktomi, the Wolf/(ves), and the Ducks play the role of primary characters, whereas Skiská and the Tree(s) play secondary roles. I will elaborate more on the important secondary role of the Tree(s) and the role of the Wind and Fire as elemental forces in Iktomi's adventure in Chapter 5. 
IKTOMI: A CHARACTER TRAITS ANALYSIS

\section{Character code distribution by version.}

The character codes break down slightly differently for the three different versions of the story, again referred to respectively as $\mathrm{Z}, \mathrm{D}$, and $\mathrm{W}$ representing Zitkala $\breve{S a}$, Deloria, and Walker.

With first regard for the two disqualified character candidates Wind and Fire, the coded data resulted in contrasting portrayals among the three versions. Z portrays the Wind as quite life-like according to a Western orientation, hinting at an ability to act willfully (Ab10), as when it stirs the Fire, plays with the Tree(s), or pulls the branches apart. Both $\mathrm{Z}$ and $\mathrm{W}$ ascribe skilled ability (Ab7) to the Wind in pulling branches apart, as well as the ability to self-locate (Ab9), that is, to move about under its own power. Conversely, D does not provide any information about the Wind beyond its mention as something other than human (Rp1) and its imminent presence (At11). D does not offer any explanation about Wind's mode of mobility — certainly nothing that could suggest it might be a personified living entity interacting with other characters.

The narrators of all three versions provide even less information about the Fire, designating it only as a non-human entity (Rp1). D and W ascribe no other traits to Fire; however, $\mathrm{Z}$ suggests in one instance each that the Fire possesses a sensory ability (Ab8) with the capacity to die (i.e., cease to live, as with a dead fire) and locomotive ability (Ab9) demonstrated as leaping flames. I discussed these examples previously and ruled them out as qualities characteristic of an animate, interactive, or motivated participant in the story. 
The versions show more consistent results for the remaining five characters. In all three versions, Iktomi is the only character that exhibits occurrences of all 15 codes in the three categories representation, abilities, and attributes. He also carries a greater percentage of occurrences than any other character of all the codes except for Rp4:Other pronoun and At11:Appearance, personality, and inclination, which are dominated by the Ducks. Because of Iktomi's predominance as a character, he is most assuredly the protagonist, and we view him shifting in form between nonhuman and human in possession of a full range of human characteristics.

Like Iktomi, the Ducks also exhibit traits consistently among the versions for all codes excluding three: (1) they are not aware of their vulnerability (Rp6); (2) they are not possessed of willful intent and, therefore, are not intentionally motivated (Ab10); and (3) they are not self-aware (At15). All the versions narrowly refer to the Ducks using language associated with humans with subtle references such as "singers, folk, dancers" (Zitkala $\breve{S a}$ ), "dancing females" (Deloria), and "youngsters" (Walker). Only in Z are the Ducks acknowledged as distinct individuals as Iktomi addresses them by kinship terms (Rp5); neither D nor W gives the Ducks the same individualizing recognition.

Character codes are also relatively consistent among the three versions with regard to Skiská. While Z, D, and W all acknowledge Skiská individually with masculine personal pronoun references (Rp3), as with the Ducks, Z also acknowledges and recognizes Skiská's individuality by giving him his own name (Rp5), albeit the name of his species_- "wood duck". In D and W, the narrators do not give the individual duck a proper name but refer to it as "one" (of the Ducks). For uniformity in identification of 
roles, I have assigned the character name Skiská as my reference to the individual duck in all three versions since all three represent the character consistently as the same individual. We find the only reference to Skiská as a specifically animate being in $\mathrm{Z}$ where he is explicitly referred to as a "duck" or a "skiská" (i.e., Rpl:Nonhuman; $c f$. lines $4 \mathrm{c}$ and $12 \mathrm{c}$ ). None of the versions depict Skiská as cognizant of his own vulnerability (Rp6), skilled (Ab7), or disposed with attributes of behaviorisms, attitudes, or selfconcern.

The three versions also portray the Wolf/(ves) consistently, with a few exceptions. Overall, the Wolf/(ves) display all trait codes except for two: they have no sense of vulnerability (Rp6), and they exhibit no positive behavior or attitude toward others (At14). Z's Wolves seem at times both human and nonhuman; D only hints at humanness with references to the Wolf's dead body since a dead animal might more likely be referred to as a "carcass" than a "body" (lines $24 \mathrm{a}$ and $25 \mathrm{~m}$ ), while W portrays his Wolf as strictly animal. In Z and W, Iktomi addresses the Wolf/(ves) familiarly by proper name or kinship term (Rp5; cf. lines 18m, 22f, and 25h), which is notably interesting for $\mathrm{W}$ whose narrator regards the Wolf as animal; these designations would normally suggest the entity is human. Both D and $\mathrm{W}$ also attribute a measure of egocentrism or self-awareness (At15) to the lone Wolf, as it talks or mulls over ideas to itself. In contrast, $\mathrm{Z}$ depicts a lead Wolf speaking to the other Wolves in a pack although in an inclusive manner for collective benefit and not out of sole interest for its own selfish purposes. The act of speaking and the mention of a pack combine to illustrate the continuum between humanness and non-humanness along which the Wolf/(ves) are 
represented by $Z$. The highlighted significance of this notion of nonhuman versus human quality, particularly in the three main characters Iktomi, Ducks/Skiská, and Wolf/(ves), will prove to be quite important to the meaning encoded in the story, and I will return to discuss it in Chapter 5.

As seen from this overview of the data, the coding suggests the characters of the Ducks and Skiská, taken jointly, parallel Iktomi’s character without his ego or selfawareness. Coding for the Wolf/(ves) also closely parallels the Iktomi character but includes the attribute of egocentrism. Whereas Iktomi and the Ducks along with Skiská display both positive and negative behaviors and attitudes toward each other, the Wolf/(ves) exhibit only negative behaviors and attitudes toward others. Iktomi must carry full responsibility for his actions, behaviors, and attitudes, as he is endowed with a full range of abilities and attributes. However, because the Ducks and Wolf/(ves) apparently lack self-perception, we might surmise they possess a certain measure of innocence, and we cannot, therefore, hold them accountable for their actions, behaviors, and attitudes. In this regard, we may consider their roles as aligned but supportive and subordinate to Iktomi's role.

Some significance must ensue from the role of Skiská and the lead Wolf as “spokesmen" for their respective groups in Z's version, as it seems important that Zitkala $\breve{S a}$ calls our attention to these standout figures which Deloria and Walker did not incorporate. Given the amount of detail that proliferates through Z's version, these conspicuous figures probably serve a special purpose unique to Z's version. I will return to this matter later in the chapter. 
With regard to the last remaining character, coding for the Tree(s) produced some interesting results. $\mathrm{Z}$ alone presents her single Tree as distinctly human, referring to it as an "old man." Z's Tree also cries out with a plea for help, which gives the Tree(s) the human ability to speak, although it does not address anyone in particular. D and W make only nonhuman references to the Tree(s); however, they likewise indicate their Tree(s) project willful intent (Ab10), which is a decidedly human trait. Furthermore, D and W give the Tree(s) individual recognition through kinship address (Rp5), which $\mathrm{Z}$ does not do. All three versions ascribe sensory, rational, and emotive capabilities (Ab8) to their respective Tree(s), which cry, speak, or squeak. $\mathrm{Z}$ also gives the Tree motion ability (Ab3) to sway as if bending and flexing under its own power; in D and W the Tree(s) are moved by an outside force - the Wind. Anaphoric references to the Tree(s) also vary: Z's Tree speaks in the first person singular though, again, it addresses no one in particular; Iktomi addresses the Tree(s) in D in the second person plural, though the tree(s) do not respond; meanwhile, W's Tree(s) stand mute as Iktomi talks about them to himself, referring to them in the third person plural.

These data combine to indicate a complex figure in the Tree(s) that the narrators themselves present from varying perspectives. In $\mathrm{Z}$, the Tree stands individually apart from the other characters and invites assistance from the first person point of view. D's version presents the situation of the Tree(s) from an outsider viewpoint - that of assistance being offered but not accepted. W's version presents a disconnected and impersonal point of contemplation, viewed through the third person, of what to do about the Tree(s)' experience of difficulty. These three perspectives in combination present the 
seeker's situation from three vantage points which are adaptable to the situation and need and provide an inescapable means for members of the audience to confront their own circumstances and thereby assume responsibility for their own actions.

\section{Summary of code distribution findings.}

To summarize the findings pertaining to character roles, I categorized each of the characters with regard to primary, secondary, or noncharacter status. Each figure designated as a character serves as a focal point of a story situation or event, projects individual identity, and engages interactively with at least one other character. Primary characters help move the story along and interact with one another in reciprocal fashion, while secondary characters play agentive thematic roles but do not engage reciprocally with any other characters, including Iktomi.

Character trait analysis fixes Iktomi as the central character and protagonist acting as motivator of all the events in the story. In the role of leading primary character, Iktomi's character progresses the story plot with the assistance of the two additional primary characters - the Ducks and Wolf/(ves). Iktomi motivates the other characters, and, together, they move events along through their actions and interactions with each other and with the environment. The Ducks and Wolf/(ves) act reciprocally with Iktomi as his subordinate counterparts in the guise of ally, supporter, or adversary. These characters do not initiate activity or motivate story events by themselves but respond to impulses coming from the protagonist. The Ducks and Wolf/(ves) dialogue interactively with Iktomi or respond to his overtures, whereas conversations and interaction between Iktomi and other perceived entities (e.g., tree, wolf's pelt) are clearly unidirectional. 
Furthermore, anaphoric reference to both the Ducks and Wolf/(ves) using third person singular masculine pronouns (he/him/his) elevates these characters' status to at least animal (specifically male), if not human. Minimally, it suggests that personification (i.e., human-like rendering) of these characters occurs to some degree and more closely aligns their characterization with Iktomi; although, again, the pronoun references pertain only to the individual duck and wolf singled out from among the larger groups.

As a secondary character, Skiská speaks out as the spokesperson for the collective group of Ducks, which momentarily personalizes them as a valid force to contend with. Yet, aside from giving the Ducks an individual voice, Skiská recedes into anonymity after his brief cameo role has been fulfilled. Skiská cannot be deemed a true primary character in his own right but, instead, as an individuated personality among the collective group of Ducks, thus demoting him to secondary status.

Just as Skiská represents an extension of the group of Ducks and serves as their mouthpiece, the lead Wolf does so for the pack of Wolves in Z's version. As such, they play secondary character roles, and neither Skiská nor the lead Wolf individually motivates the story progression.

I pause to note that it would seem I have neglected to identify the figure of the lead wolf as a potential character, since I compare it to Skiská. I justify this omission on account that $\mathrm{D}$ and $\mathrm{W}$ incorporate a single wolf, while only $\mathrm{Z}$ mentions multiple wolves comprising a "pack.” Despite Zitkala S̆a's suggestion of plurality, only one individual essentially fulfills the role of the Wolf/(ves.)The pack does not perform any acts, speak, or engage in any way apart from Z's simple mention of their existence whereas, in 
contrast, $\mathrm{Z}$ describes the Ducks as a group made up of individuals who dance, sing, and carry on throughout the whole scene with Iktomi. Therefore, although I use the designation "Wolf/(ves)" as a means to inclusively refer to the character, including the lone wolf and its cohorts as represented in the three versions, the character of the Wolf/(ves) is represented clearly as a single unit, whether as a lone animal or as a pack that acts as one.

The Tree(s) play dual roles teetering between thematic agents in a secondary character capacity and then as a non-character prop. The Tree(s) first seem animated with conscious personality but then revert to inanimate figures as Iktomi climbs up and gets himself stuck in their branches. As the former, Iktomi addresses the Tree(s) with kinship terms, for example, "Little Brothers" in D and "Younger Brother" in W, and the Tree(s) provide a focal point of conflict with the protagonist Iktomi. As the latter, because the Tree(s) do not actively participate in the conflict with Iktomi but are only the unresponsive target of his machinations, they cease in their role as a real character in the sense used here. Rather, they become a plot device, similar to the nature of ducks as game animals, as I mentioned in the literature review.

The noncharacters Wind and Fire function as accessories to the main action of the storyline. They do not mindfully interact or engage with the characters but serve as aids and instruments to help the characters accomplish their objectives or affect conditions through the story's course of events. The Wind, at first, seems animated and selfmotivated, but it does not interact in a conscious way with any other characters or props. Despite Z's subtle suggestion to the contrary, the Wind does not exhibit willful intent to 
act; rather, its affect on other elements in the environment is incidental of its natural undirected movement and not that of a conscious entity.

Likewise, the Fire represents an elemental force lacking conscious motivation. Its role in the story essentially functions as instrument, which Iktomi uses and directs to effect a changed condition on other items (e.g., render food from the raw to the cooked state). Since the evidence in the data coding does not suggest that either Wind or Fire act with conscious intent, I have not treated them as characters in the analysis.

A final note of curious interest on character portrayal in the three versions pertains to how the characters of the Ducks and Wolf/(ves) are transformed through story development. Similar to the way the Tree(s) move from inanimate beings to animate beings and back again, likewise, the Ducks and Wolf/(ves) shift from consciously motivated individualized entities endowed with human-like qualities, vigilant and aware of their environment, first, into non-rational instinctual animals, and then into objects dispossessed of individual personality, indifferent to their surroundings and eventually lifeless and lacking a spirit. This peculiarity reinforces the essence of duality repeatedly highlighted throughout the tale: the dual nature of humankind; consciousness of right and wrong; individual versus group; two trees/two branches; raw/new state versus finished/completed state, and so on. I will also return to discuss the nature of this duality in Chapter 5.

\section{Phase 2: --Polarity-code correspondences.}

Organizing the codes with regard to polarity-code correspondences provided additional quantitative measures from a different perspective for ascertaining the nature 
of the characters and comparing their roles and relationships among the different versions of the Iktomi story. As described in Chapter 3, the polarity-code correspondences sort the accumulated character traits codes into diametrically paired oppositions which differentiate subtle contrasts among the different characters.

After having identified and defined the characters using the simple word counts of traits codes occurrences, I gleaned additional support for character identification from the polarity-code correspondences. The polarity-code correspondences also served to position the characters relationally to one another according to their described natures. Contrasts observed in the data drew clearer distinctions about the nature of the characters as the narrators portrayed them while highlighting key elements which audience members might be able to identify and recognize as intrinsic to their own nature.

I present the results of my analysis of the polarity-code correspondences derived from the character traits codes data in the following sections, using the Polarity CodeCharacter Code Correspondences Template in Appendix D as a reference. The template indicates the corresponding links between coded character traits and the paired polarity groups in composite for the five characters identified in the previous section. Wind and Fire are not relevant to this discussion since I have eliminated them from the lineup of characters.

\section{Polarity-code data analysis.}

I conducted my analysis of the polarity code data employing the calculated weighted and unweighted polarity-code values detailed in Chapter 3 (cf. Tables 4 and 5). I present the results of my findings, first, as an overview of the weighted and unweighted 
values then, with a more in-depth look at the contrast among the paired sets of polaritycode groups. Both types of composite values provide a general overview of the story's composition, while calculations for the individual versions allowed me to ascertain how the different versions contrastively present their characters.

Overview of polarity-code correspondences results.

Whereas a single instance of a character trait in a particular polarity-code group automatically ascribes that quality to the character, the frequency of such occurrences indicates the strength or degree of influence of that quality's presence in the character's nature. The relative frequencies pertaining to different characters also position the characters relationally, revealing how they interrelate or correspond to one another.

In analysis, the data based on simple word counts reflect a consistent pattern of distribution of the codes for the different characters in all three versions with variation arising only in the extent of code frequency. These data provide evidence for my claim regarding the status of the roles played by Iktomi, the Ducks, Skiská, the Wolf/(ves), and the Tree(s).

The unweighted values reflect the actual accumulations of the code occurrences for the characters and showed the extent to which the narrators elaborated with descriptive details and the range of variation between versions. They also provided clarity pertaining to the versions' depiction of characters relative to one another.

The weighted values normalize the values distributed among the characters in order to compare them on an equitable scale. The weighted values reveal subtle nuances of apparent narrator stance in their portrayal and emphasis on different characters, which 
I found suggestive of specific directed narrative intent. As stories can have a variety of purposes, I viewed these subtleties as providing clues about the nature of the circumstances surrounding the specific storytelling event that may have prompted the particular outcome of narrative content.

In analysis, both weighted and unweighted code frequency distributions reflect consistent development of characters among the different versions of the story and undeniably, all three narrators present Iktomi as the most central character to the storyline. The results from both Tables 4 and 5 indicate that the characters Iktomi and the Ducks display the greatest share of coded traits of all the characters in nearly every polarity code group. The Wolf/(ves) also make a fairly strong showing of codes in most groups, while neither Skiská nor the Tree(s) score comparably to the other three characters in most groups on either scale. Skiská's consistently low scores in each group reinforce my earlier conclusion that the figure acts as an extension of the collective group of Ducks rather than as an independent character in its own right. The Tree(s) figure similarly to Skiská, except that no other group of trees exists with which they might share similar communal affiliation, such as Skiská shares with those of his own kind. This singularity of the Tree(s) impels me toward my earlier speculation that the Tree(s) seem to play a special role differentiating their nature from the other characters.

When the weighted values of the data are viewed on the relative scale, they indicate that the narrators described the characters with varying degrees of emphasis, suggesting the storytellers may have considered the characters in a ranked priority of importance with regard to their complementary roles. A comparison of the relative 
scores for the characters in each of the groups for the three versions reveals certain tendencies of descriptive emphasis toward the different characters:

Zitkala S̆a and Walker used more descriptive detail with regard to Iktomi than Deloria;

Zitkala S̆a ascribed more descriptive traits to the Ducks than Deloria and Walker, who described them in relatively equal measure.

Walker provided more description of the Wolf/(ves) than Zitkala S̆a and Deloria, who described them as closely the same.

Aside from more distinctly emphasizing the Iktomi character in all three versions and spotlighting his protagonist role, based on scores of the weighted values of polaritycode occurrences for the other four characters, these observations also suggest the narrators may have favored one of the remaining characters over the others in a certain way. Zitkala S̆a's description tends more heavily toward the Ducks than either Deloria's or Walker's version, while Walker's description leans more toward the Wolf/(ves) than Deloria's or Zitkala S̆a's version. Deloria does not appear disposed one way or the other. I would like to suggest here that the subtle preferences shown by Zitkala S̆a and Walker add evidence to support and clarify the roles of the Ducks and Wolf/(ves) in the story as being representative of feminine and masculine aspects of character, respectively, through natural affiliation with the sex of the individual whose lens the story filters through to us. The preferences also lend support to the claim that the characters in this Dakota story stand as representatives of traits and qualities that audience members can identify with and recognize in themselves. I will address the significance of these narrator preferences and the feminine/masculine component of the characters, including Deloria's apparent neutrality later in this chapter. 
Analysis of polarity-code pairs.

In this section I describe the polarity-code results with regard to the group pairs and discuss what they may relate about the roles of the characters. According to the polarity-code groupings, all three versions describe the characters consistently with regard to the ten polarity-code types; however, some differences occur between the weighted and unweighted results.

Both the weighted and unweighted data in the first set of paired polarity codes show that each of the characters appears as both nonhuman and human. Because the characters exhibit traits that indicate the possibility of existing concurrently as both nonhuman and human, consideration of cultural perspective helps elucidate and reconcile the apparent contradiction. Dakota tribal lore regards the character of Iktomi as a cultural trickster that takes the form of a spider though also as a shapeshifter which can change into human, animal, or other forms. The Ducks and Wolf/(ves), of course, are animals; however, in the story, they are also capable of speaking and thinking rationally as humans do. Further, the ohuykakay tales, by definition, are thought to originate from a time when animals and humans were alike and animals possessed every human capability. Consequently, on the one hand, we can acknowledge that all the characters in Iktomi represent a living being in the form of an animal or plant; on the other hand, in accordance with the notion of a prehuman era in myth time, we can also accept the characters' analogy to aspects of humanness.

All three narrators appear to acknowledge this "same-but-different" aspect of the three main characters, Iktomi, the Ducks, and the Wolf/(ves), as animal characters, yet 
they also stress the three characters' humanness by ascribing substantially more humanly descriptive traits to them than to the other characters. The descriptions of Skiská and the Tree(s) emphasize humanness less so, offering the briefest suggestion that the two characters bear similarity to the other three. The characters' roles explain and justify the differences in emphasis, as I have already explained. Because the Ducks and Wolf/(ves) represent facets of character recognizable to audience members, it stands to reason that their humanness should be more pronounced, while Skiská's role as spokesperson for the Ducks remains minimal and momentary, and the Tree(s) stand as a symbol of choice rather than of a particular individual.

Aside from the first dichotomous pair of nonhuman and human just discussed, differences in character portrayal among the versions emerge through both the unweighted and weighted values of grouped traits in each of the remaining polarity sets. The unweighted data show more variation among the characters and highlights their differences and distinctions in relational positioning. The weighted data tend to merge the characters' qualities into unifying themes that link the characters ideologically to one another and hint at more subtle connectors among them.

With regard to the unweighted polarity-code data, the second set of conscious and unconscious groups indicates two differences in the code frequencies: first, whereas Deloria and Walker portray the Ducks as not conscious, Zitkala S̆a does the reverse; second, Zitkala S̆a's version depicts the Tree(s) as primarily unconscious, Walker's Tree(s) are conscious, and Deloria makes no distinction of predominance of one quality over the other. These differences support apparent favor toward the Ducks character as a 
key player in Zitkala S̆a's version of the story, while emphasis on the Tree(s) in Walker's version bolsters support for their role as signifying a particular purpose in his narrator's eyes.

Also relative to the unweighted data, Iktomi and the Wolf/(ves) in all three versions show a greater tendency toward consciousness — Iktomi dramatically so. Consciousness points again to the human and animal capability to perceive and interact with other elements. The strength of Iktomi's rating indicates that he much more actively engages and responds to external stimuli at a rational level of awareness than any other character, reinforcing his role as the protagonist and a figure with which a human audience might easily identify. The Ducks' more marked exhibition of unconsciousness qualities accentuates the automaticity of their behavior. The weight of their unconsciousness is also underscored by the fact that, for that character, only one type of character traits sorts into the unconscious group compared with six types of character traits for the conscious group (cf. Appendix D). This set of polarity codes indicates the Ducks tend to more spontaneously and mindlessly react to external prompts in the environment while exposing certain characteristics of which they may not be entirely aware. In contrast, the Wolf/(ves)' slightly more conscious qualities elevate their level of awareness of their own nature, indicating cognizance, mindfulness, and reasoned response on their part.

The distribution of unweighted values for group and individual orientation codes yields somewhat surprising results. Iktomi and the Wolf/(ves) exhibit more individual focus as expected; however, the Ducks and Skiská also tend slightly more toward 
individual orientation while the Tree(s) lean slightly more toward group orientation. We might expect the solitary figure of the non-interactive Tree(s) to tend instead toward individual orientation, while individual orientation seems contrary to the basic flock nature of ducks. Only Walker's version ascribes the Tree(s) with more individual orienting traits, reiterating the stand-alone importance his narrator imparts to them. The anomalous results imply a duality in the nature of the Ducks and Tree(s) characters that allows them to project qualities antithetical to their natural state of being and suggest something else is going on here. The Ducks' individualism seems to indicate identification or alignment with singularity in the sense that they represent an aspect of character that I would argue coincides with the protagonist Iktomi and, by extension, the audience. The impersonal Tree(s) orienting toward group identification seem more universal, identifying not with specific individuals but, rather, representing a collective concept available to everyone. This perspective fits with the idea that the Tree(s) signify a universal crossroads of choice where the individual must confront the duality of his own mind and decide on a course to follow.

The Wolf/(ves) less surprisingly come across as more individually oriented, bringing to mind the concept of the "lone wolf." However, this orientation also indicates an aspect of the Wolf/(ves) character in the same way as for the Ducks in mirroring for Iktomi as well as for members of the audience. The individually oriented Wolf/(ves) serve as a reminder that the seeker's quest is a personal journey that must be undertaken alone and that his or her decisions in life are his or her own to make. 
Iktomi's slant toward individual focus reinforces his role as the main character and focal point of all the events in the story, situating him as the prime representative of the individual self. It also reiterates a focus on the male self or aspects of the masculine that reside within every individual—male or female. That Iktomi also exhibits a fair share of group orientation primarily reflects his attempts to identify with the group of Ducks in order to obtain in-group status, gain their trust, and thereby position himself for exploiting them. In parallel to the story, individuals need to self-identify as belonging to their social group, that is, their family, community, and tribe, in order to meet their basic needs for survival and overall physical, mental, emotional, and spiritual good health. Similar to the Tree(s), the duality that may also be implied through Iktomi's group orientation indicates the trickster's adaptive ability to reflect a variety of contradicting aspects for any individual—male or female.

I add one final note regarding the group focus/individual focus pair: although substantially more types of character traits codes sort into the individual focus group, the number of actual occurrences of the codes does not reflect a bias attributable to an imbalanced distribution. Instead, certain traits codes simply occurred with more frequency than others, making up for the difference as reflected in the equalized distribution. In other words, the number of code occurrences balances out the variability of the codes figuring into the group.

In the next paired set of polarity codes, all characters in all three versions display higher weighted percentages of intentional-willful qualities over passive-receptive qualities, due in part to the greater number of traits codes that fall into the intentional- 
willful subcategory. However, each character shows essentially comparable unweighted scores between intentional-willful and passive/receptive inclination which may attest to the characters' shifting roles as they progress through the story. Or the scores may reflect their changing behaviors and attitudes which vary depending on the other different characters they engage with. For example, shifts are evident through interactions between Iktomi, the Ducks, Tree(s), and Wolf(ves): first, Iktomi coerces the Ducks through willful cunning and persuasion, and the Ducks respond to him passively until they discover he is killing them, after which, they take a more proactive stance; second, Iktomi exerts his will toward the Tree(s) but then shifts toward passivity while held captive by their pressed branches; third, the Wolf/(ves) exhibit both tendencies as, on the one hand, they passively avoid direct confrontation with Iktomi, yet on the other hand, they proactively take advantage of opportunity when it arises.

Finally, in accordance between the unweighted and weighted polarity-code groupings, the results consistently show preference in the named subcategory over the unnamed subcategory for all the characters except for the Ducks. The Ducks' results in the Named/Unnamed group reflect that they go decidedly unrecognized from a personal standpoint by other characters. The point underscores their apparent distancing from or non-identification with other characters, and I would argue it reinforces the contrast between the female nature inherent in the Ducks character as compared to the male nature of all of the characters in general. The implication suggests that while both men and women possess feminine as well as masculine attributes, as warriors and hunters for whom strength, power, and bravery were paramount, Dakota males such as those in the 
audience would less likely have resonated and identified with those qualities more associated with the women who depended on them for provision and protection. The named/unnamed group also, once again, associates the various characters with humanness through the traits they exhibit, namely those coded Rp2:Human and Rp5:Recognition. The Ducks entertain only a few references, none by name, referring to them as singers, dancers, folk, friends, females, or youngsters. These epithets certainly cause one to think "human" despite only rather impersonal acknowledgement accorded to the Ducks in the story.

Comparison of weighted percentages in the subcategories aside from nonhuman and human within each paired set shows each character to be primarily conscious, individually oriented, intentional-willful, and named or recognized entities. The only exception to consistent portrayal evident in the weighted data concerns the Ducks, since in Deloria's and Walker's accounts they are the only character that is not personally acknowledged or named by some other character. Their non-recognition in those two versions suggests the protagonist distances himself somewhat from them and does not self-identify with them to any great extent. Zitkala S̆a's characters, including the Ducks, all seem to interrelate, which may possibly reflect her personal views on humanitarian inclusiveness or a personal attempt to convey the Dakota social value of "respect for others" by acknowledging every individual, regardless of status. The similarity of polarity-code focus among the characters throughout the three versions strongly suggests relatedness among the characters in the sense that they identify with one another in a certain manner, and these findings support my assertion that the supportive characters 
represent aspects of the main character which, in turn, represents the seeker on vision quest.

\section{Summary of polarity-code correspondences: Character roles and relationships.}

Analysis of the data shows support for identification of the story characters with their human audience and establishing links between the characters' qualities and behaviors and those of audience members. The Ducks' and Wolf/(ves)' relationship to the protagonist Iktomi plays out quite differently and traces back to their association with the feminine and masculine qualities. The dual nature of the three primary characters, that of being both human and not human at the same time, serves as a reminder that the characters represent different sides to the individual's nature which must be acknowledged and dealt with. One might equate the nonhuman side with the individual's unconscious state, that is, those aspects of personal character that one does not wish to acknowledge in oneself.

The unconsciousness traits speak to the innate qualities and inclinations inherent to the characters in all their representations as human, animal, or inanimate entities and also to those qualities that reside in the individual's state of mind below the level of awareness.

Group orientation reflects the need to be accepted into one's social organization for emotional security and general well-being, while individual orientation reiterates that one must ultimately take responsibility for one's own choices and decisions in life. Traits in the passivity-receptivity grouping indicate the characters' emotional response and/or attitude toward environmental stimuli or situations and indicate a measure of 
powerlessness over their own circumstances or non-proactive acceptance of the situation. The narrators show Iktomi to be far more responsive to his environment than other characters, again, reiterating his universal status among the three versions as primary character and protagonist.

\section{Character summary.}

Analyses of character traits and polarity-code correspondences together have shown support for the key points I set out to demonstrate at the beginning of this chapter. In all three versions, self-will and personal gratification motivate Iktomi's actions. Iktomi's self-concern suggests he may not be truly unconscious of his intentions, and acknowledgement of him as an individual further supports his characterization as actor, agent, and motivator of the story's events while rationally proactive in his own schemes.

Only in Zitkala S̆a's version are the Ducks personally acknowledged when Iktomi addresses the group of Ducks with kinship terms; both D and W simply refer to them impersonally as objects and prey.

Likewise, only Zitkala S̆a calls up the issue of individuation and recognition of a duck singled out and possessing his own identity; Zitkala S̆a provides an opportunity for an individual voice to express itself, whereas Deloria and Walker offer no distinction from the unindividualized group. Attention to recognition through naming in Zitkala S̆a's version suggests a spotlighted direct and focused purpose for the Skiská character which could be linked to Zitkala $\breve{S} a$ 's role in life as activist and spokesperson for Indian human rights. 
With regard to the Wolf/(ves), Walker's version contains greater frequencies of code occurrences in the named/unnamed groups which are probably distorted due to the extended ongoing encounter between Iktomi and the Wolf occurring in Episode 4.

The Tree(s) stand out as more individually oriented in Zitkala S̆a's version, although she does not treat them as an individual personality. The discontinuity may be explainable as Iktomi's projection of his own consciousness onto the tree and his attempts to interact with it on an equal footing.

All of these points support Iktomi as the main character with the Ducks and Wolf/(ves) serving to highlight aspects of Iktomi's projections of self and, consequently, as projections of the audience members' selves. Skiská and the lead Wolf act as singledout representatives for their respective communal groups, giving vocal expression to those aspects of self that normally remain hidden, i.e., the masculine and feminine nature of one's own being. The Tree(s) stand neutrally as a universal iconic bridge between the higher and lower realms of conscious awareness and as the nexus between the material and spiritual dimensions of existence. As such, it signifies the juncture at which one must make a decision about which course to follow in one's life journey.

\section{Phase 3: Pronoun analysis.}

In this section of the data analysis, I present descriptions and findings regarding the narrators' use of Dakota enclitics and English pronouns and evidence that leads to the story's focus on a male theme. Then I will give support for inferences I made from the data and explain how I arrived at my conclusions. 


\section{Enclitics as indicators of biological gender.}

As prefaced in Chapter 2, Trechter (1996) and others explained that Dakota enclitics mark a variety of nuances that communicate information about stance and emotional expression while also indirectly indexing gender of the speaker, among many other subtleties intended by the speaker and warranted by the circumstances (pp. 100116). My study concerns the referential indicator of sex of the speaker.

To find out whether the enclitics present in the narrative could provide clarity about the characters' sex, I examined Deloria's Dakota language transcription to ascertain which enclitics the characters might use in active speech. I found that four of the five characters actually do use such enclitics: Iktomi uses yelo, welo, huwo, po, ye, and we; the Ducks employ lo, huwo, and ye; the Wolf/(ves) use lo; and Skiská uses po and lo. Table 7 lists the enclitics the characters use.

Enclitic use does not apply to the Tree(s) which do not directly interact with any other character or respond to Iktomi's pleas, much less speak. All of the enclitics otherwise employed by the characters are those associated with male Dakota speakers. 
Table 7.

Characters' Use of Enclitics in Deloria's Dakota Version of Iktomi Text

\begin{tabular}{|c|c|c|c|c|c|c|c|}
\hline \multirow[b]{3}{*}{ Character } & \multicolumn{7}{|c|}{ Function / Enclitics } \\
\hline & \multicolumn{3}{|c|}{ Assertion } & \multirow{2}{*}{$\begin{array}{c}\text { Interrogative } \\
\text { huwo }\end{array}$} & \multirow{2}{*}{$\begin{array}{c}\text { Imperative } \\
\text { po } \\
\end{array}$} & \multirow{2}{*}{$\begin{array}{l}\text { Request or Endearment } \\
\text { ye/we }\end{array}$} & \multirow{2}{*}{$\begin{array}{c}\text { Assertion } \\
\text { ye/we }\end{array}$} \\
\hline & lo & yelo & welo & & & & \\
\hline Iktomi & & $\mathrm{x}$ & $\mathrm{x}$ & $\mathrm{x}$ & $\mathrm{x}$ & $\mathrm{x}$ & $\mathrm{x}$ \\
\hline Ducks & $\mathrm{x}$ & & & $\mathrm{x}$ & & $\mathrm{x}$ & \\
\hline Skiská & $\mathrm{x}$ & & & & $\mathrm{x}$ & & \\
\hline Wolf/(ves) & $\mathrm{x}$ & & & & & & \\
\hline Tree(s) & & & & & & & \\
\hline
\end{tabular}


Only three instances of enclitic use occur in the text which could potentially indicate a female speaker and involve ye/we. The enclitics ye and we are normally used by women to indicate imperative, opinion, or permission, yet men may also use them as markers of entreaty or endearment as when speaking to a child. In another context, both men and women use ye/we to energetically express assertion (Trechter, 1996, 35).

In the text, Iktomi employs ye as an assertion as he congratulates himself on his successful bagging of the Ducks for food and we when addressing the Tree(s) endearingly as his younger brothers, while the Ducks use ye when entreating Iktomi to sing for them. I found no occurrences of enclitic markers of strictly female speech in the texts, although all of the cited sources list such enclitics. Therefore, I conclude with confidence that enclitic use in the Dakota-language version of the narrative confirms the male sex of the four interactive characters. Although male sex of the Tree(s) cannot be included through this discussion, the fact that Iktomi refers to them as "little brothers" is itself indicative of their male status.

Because Deloria's text is the only one we have available in the Dakota language, I was unable to make comparable observation of the other two versions. However, since each version has been rendered with an English translation, in addition to noting the existence of other confirming indicators of male sex of the characters, I have chosen to perform an analysis based on the use of English pronouns found in the translations, assuming those translations maintain the integrity of the original meaning that was intended by the narrators. 
IKTOMI: A CHARACTER TRAITS ANALYSIS

Results, Analysis, and Findings of Pronoun Use.

From the tabulated information regarding pronoun use, I found certain patterns emerged from the distribution of anaphoric reference to each character which offered insight about the characters' nature, role, status, function, and significance to the storyline as well as the relationships between the characters and their environment and story props, all occurring within the story context while relating to the audience outside the story proper.

The relative numbers of occurrences for the characters seemed particularly relevant to reaffirming the relative bearing of the characters to the storyline. Just as importantly, incorporation of pronouns, whether occurring as single or multiple instances of use, demonstrated the elements of character that helped clarify the characters' natures. For example, masculine pronoun references confirm the male sex of the character in question, and in turn, they denote animate quality. Although we cannot definitively distinguish the character as human or animal by the use of English pronouns, the substantiality of their number of occurrences could suggest that they more pointedly denote humanness.

When characters other than the one referenced use personal pronouns to refer to another character, they acknowledge their fellow as a figure to be reckoned with, perhaps as a peer, and one with whom they can identify in some way. Such personal pronoun references imply a quality of humanness possessed by the referent; therefore, the resemblance of the characters to humans most significantly factors into the audience's ability to identify with the characters and assume their characteristics as their own. As 
the characters identify with one another, so members of the audience may similarly resonate with each of the characters and their respective behaviors, thoughts, and attitudes.

The narrators' particular use of masculine pronouns in reference to certain characters, as with direct kinship references, serves two purposes: first, it helps to raise the status of the character from inanimate to animate and possibly human, thus marking the relative importance of its role; and second, these pronouns indicate to the audience how they should relate to the figure in terms of kin relationship and to the story characters' actions as compared with their own in real life.

As indicated in Chapter 3, I divided the pronoun data into two groups: first, I focused specifically on third-person-singular-masculine pronouns and then, second, I considered the accumulated instances of all other personal pronouns. I present the results of my analysis and findings pertaining to the separate groups in the next sections.

Third-person-singular-masculine pronouns - he; him; himself; his.

In English, third-person-singular animate pronouns index human and animal qualities in the nouns they reference; therefore, their use in the story context reveals information about the personal identity of the character as an individual, bringing it to life and acknowledging it as a distinct personality. It also helps with discerning the relationships between characters by emphasizing their rank of importance to the plot and shows whether the characters relate to one another on a higher, lower, or equal level, if animacy can be related to status. The distribution of these pronouns in terms of percentage frequency or relative numbers of occurrences through their use in the different 
versions reveals how the narrators apparently perceived the characters and described their relationships.

In the English renderings of the versions, the three documenters used the thirdperson-singular-masculine pronoun forms he, his, him, and himself in reference to the various characters. Tabulating occurrences of these pronouns, I found instances of use for the characters of Iktomi, the Ducks, Skiská, and the Wolf/(ves); as with the enclitics, none of these pronouns occurred in conjunction with the Tree(s). Table 8 displays the distribution of these pronouns grouped together for each of the characters as occurring in the three versions.

\section{Table 8.}

Character Distribution of Third Person Singular Masculine Pronouns

\begin{tabular}{lcccccc}
\hline \multicolumn{7}{c}{ Number of Occurrences of Pronouns Used to Reference Character } \\
he, his, him, himself
\end{tabular}

The values in the table show that Iktomi figures most prominently among all the characters, obtaining 260 of the 306 third-person-singular-masculine pronoun references used in the three versions of the story. Iktomi also consistently carries the greatest weight of distribution within the individual versions. The Wolf/(ves) in both Deloria's and Walker's versions carry more weight than Skiská and the Ducks combined, while the Ducks and Skiská in Zitkala S̆a's version outweigh the Wolf/(ves). Not only does the 
weight of these numbers reinforce Iktomi's status as the protagonist, the distributions also support the assertion of slight narrator favoritism and gravitation toward a specific character beyond Iktomi. In other words, Zitkala S̆a's version, which I contend is written through a female lens, seems to lean more toward the feminine aspects nuanced through the Ducks and Skiská characters, while Walker's and Deloria's male informants appear to gravitate more toward the masculine aspects of the Wolf/(ves).

\section{Summary of findings: Third-person-singular-masculine pronouns.}

The findings from third-person-singular pronoun data analysis affirm that all three narrators recognize Iktomi as a thinking, rational, self-motivated being, specifically suggesting human characterization. While the characters in D and $\mathrm{W}$ reference the Wolf/(ves) far fewer times than Iktomi with masculine pronouns, their frequency, 9 and 17 times, respectively, in comparison with all other characters suggests the narrators perceived the Wolf/(ves) to bear similar characteristics to Iktomi and, therefore, as an entity with whom the audience could also relate and identify.

By the same logic, the narrators reference the Ducks only infrequently, 4 times in $\mathrm{Z}, 3$ times in $\mathrm{W}$, and only once in $\mathrm{D}$, suggesting a measure of relative distancing between Iktomi and the Ducks while Skiská, as the stand-out Duck, receives a few more masculine pronoun references which position him in somewhat closer proximity to the more male-oriented characters. As mentioned earlier, the fact that each character receives third-person masculine anaphoric reference indicates that all are male characters; however, I restate that their relative frequency of reference indicates differentiation in how other characters perceive and relate to them. Other characteristics in the form of 
traits and behaviors described earlier then come into play to emphasize the feminine and masculine qualities inherent in the characters, which I have proposed renders the characters more male or female in orientation.

Evidence for "male" focus of the story.

The first obvious sign of male focus in Iktomi becomes evident through incorporation of the masculine enclitics in the Dakota version and pronouns in the English versions. Feminine pronouns are not used at all in the English versions of the story, nor are there other less direct indices of female speech. Working under the assumption that the translations preserve the intended meaning in the original Dakota versions, the absence of female markers adds to the support for male focus in the story that the use of masculine enclitics attest. These masculine pronouns and enclitics in combination highlight the main character Iktomi as specifically representing a male entity, reiterate the prominence of maleness as a focus of the tale, and strongly imply that this story carries special significance for male members of the audience. A male main character would accentuate the focus of the plot as directed toward men's interests and emphasize the masculine aspect in the story's events, all of which would carry greater significance for a male audience who would be able to relate personally to such references.

That is not to say that Dakota women were culturally invisible, only that the roles of men and women were observed as distinctly separate in the culture. Tribal members recognized, appreciated, and respected the complementary abilities and qualities that individuals of each sex contributed to maintain the health and wholeness of the society. 
Aside from the absence of feminine pronoun and enclitic usage, additional support for the story's focus on men's interests issues directly from cultural practices and taboos. Ideally, the Dakota culture imposed strict rules of behavior with regard to crossgender communication and interaction in order to maintain proper distance and respect among male and female members of the society. For example, protocol deemed it improper for a man to speak directly to his wife's mother and vice versa; they could communicate information to one another indirectly through the man's wife or through their actions or conversation directed toward others within the other's hearing. Likewise, unchaperoned young women and men did not associate with one another, and they typically did not engage with one another in the same activities. The strict protocols helped maintain one's reputation as honorable, reliable, and trustworthy, which the Dakota people considered imperative for maintaining harmonious cooperation within the group. To flout the taboos would disrupt the peaceful flow of daily life and potentially bring disaster to the group if its members could not unite against outside threats. Therefore, the distinct separations drawn between the roles of males and females in the tribe served to order and protect the interests and welfare of the whole society. Everyone understood the expectations regarding their relationship to one another, and they communicated their understanding through respectful conduct and reinforced it through their discourse.

In light of all these factors, I reassert that this Iktomi story, which is centered on a male character and employs male pronoun references, likely focuses on characteristics of 
a life situation particularly relevant to males. I now turn to show how the results of data coding for other personal pronoun usage in Iktomi support my assertion.

Other personal pronouns and determiners.

Personal pronouns other than the third-person-singular cast light on other aspects of the characters' self-perception and their or the narrators' perception of the other characters. Impersonal or collective third-person pronouns create emotional distance, whereas personal first- and second-person pronouns bring the emotional atmosphere into direct contact, since they involve mutual interaction between the individual and the self or others. For instance, a character speaking in the first-person acknowledges first, itself and secondly, potentially, a reciprocal interlocutor since speech involves the speaker and the addressee directly. In contrast, third-person reference positions the speaker at a point removed from others, whether from immediate direct interaction or through separation by time and/or distance. In this case, the speaker talks about another entity without directly addressing it or involving it in a participatory speech event, while the one spoken about may or may not know what is being said about it.

Summary of findings: Other personal pronouns and determiners.

Pronominal references help illuminate the nature of the characters and their roles as they relate to the protagonist Iktomi, and Iktomi's relationship with the other characters emerges through his consecutive interactions with them.

Tables 9 and 10 itemize the personal pronouns and determiners (excluding thirdperson-singular-masculine) that occur in the texts along with their frequency of occurrence. The personal pronouns shown in Table 9 are grouped according to person 
and number and include subject, object, and possessive case; indefinite forms that occurred in reference to characters are listed in Table 10. 
Table 9.

Other Personal Pronouns-Number of Occurrences as Used by Character

\begin{tabular}{|c|c|c|c|c|c|c|}
\hline \multicolumn{7}{|c|}{ Personal Pronouns and Determiners: Subject, Object, Possessive Case } \\
\hline \multicolumn{7}{|c|}{$1^{\text {st }}$ Person Singular: I, I'll, I'm, me, my-self-referencing } \\
\hline Version & Iktomi & Ducks & Skiská & Wolf/(ves) & Tree(s) & Total \\
\hline Zitkala S̆a & 20 & $\begin{array}{c}---- \\
---\end{array}$ & ----- & $\begin{array}{c}---- \\
---\end{array}$ & 2 & 22 \\
\hline Deloria & 20 & ----- & ----- & ----- & ----- & 20 \\
\hline Walker & 17 & ----- & ----- & ----- & ----- & 17 \\
\hline Total & 57 & ----- & $\begin{array}{l}---- \\
\end{array}$ & $\begin{array}{l}---- \\
\end{array}$ & 2 & 59 \\
\hline
\end{tabular}

$1^{\text {st }}$ Person Plural: we, us, our - self-referencing

\begin{tabular}{lrrrcrr}
\hline \multicolumn{1}{c}{ Version } & Iktomi & Ducks & Skiská & Wolf/(ves) & Tree(s) & Total \\
\hline Zitkala Ša & 1 & 5 & ----- & 2 & ----- & 8 \\
Deloria & ----- & 2 & ---- & 2 & ---- & 4 \\
Walker & ----- & ----- & ----- & 1 & ----- & 1 \\
\hline Total & 1 & 7 & ----- & 5 & ----- & 13 \\
\hline
\end{tabular}

$2^{\text {nd }}$ Person Singular/Plural: you, your, yours - referenced by others

\begin{tabular}{lrrrrrr}
\hline \multicolumn{1}{c}{ Version } & Iktomi & Ducks & Skiská & Wolf/(ves) & Tree(s) & Total \\
\hline Zitkala Š & 5 & 9 & ----- & 3 & 1 & 18 \\
Deloria & 2 & 6 & ----- & 4 & ---- & 12 \\
Walker & 4 & 6 & ----- & 3 & ----- & 13 \\
\hline Total & 11 & 21 & ----- & 10 & 1 & 43 \\
\hline
\end{tabular}

$3^{\text {rd }}$ Person Singular: it - referenced by others

\begin{tabular}{lrrrrrr}
\hline \multicolumn{1}{c}{ Version } & Iktomi & Ducks & Skiská & Wolf/(ves) & Tree(s) & Total \\
\hline Zitkala S̆a & ----- & ---- & 1 & ----- & ---- & 1 \\
Deloria & ----- & 2 & ----- & 4 & ---- & 6 \\
Walker & ----- & 4 & ----- & 2 & ----- & 6 \\
\hline Total & ----- & 6 & 1 & 6 & ----- & 13 \\
\hline
\end{tabular}

\begin{tabular}{|c|c|c|c|c|c|c|}
\hline Version & Iktomi & Ducks & Skiská & Wolf/(ves) & Tree(s) & Total \\
\hline Zitkala S̆a & ----- & 21 & ----- & 6 & ----- & 27 \\
\hline Deloria & ----- & 18 & ----- & ----- & ----- & 18 \\
\hline Walker & ----- & 3 & ----- & ----- & 6 & 9 \\
\hline Total & ----- & 42 & ----- & 6 & 6 & 54 \\
\hline
\end{tabular}


Table 10.

Other Indefinite Pronouns-Number of Occurrences as Used by Character

\begin{tabular}{|c|c|c|c|c|c|c|}
\hline \multicolumn{7}{|c|}{ Indefinite Pronouns (relative, demonstrative, quantifier, reciprocal) } \\
\hline \multicolumn{7}{|c|}{$\begin{array}{l}\text { which, that, those, some, all, anyone, one, two, nobody, each other - } \\
\text { referenced by others }\end{array}$} \\
\hline Version & Iktomi & Ducks & Skiská & Wolf/(ves) & Tree(s) & Total \\
\hline Zitkala Sa & ----- & 4 & 2 & ----- & ---- & 6 \\
\hline Deloria & ----- & 5 & 1 & 4 & 3 & 13 \\
\hline Walker & ----- & 11 & 1 & 1 & 2 & 15 \\
\hline Total & ----- & 20 & 4 & 5 & 5 & 34 \\
\hline
\end{tabular}

None of the narrators in any of the versions referenced Iktomi in the third-personplural as they always considered him to be a single individual; they used only singular masculine forms (he, him, himself, his) to reference him.

Regarding pronouns other than third-person, the narrators in all three versions employed only first- and second-person personal pronouns or determiners in subject, object, and possessive case when referring to Iktomi. Right away, we begin to experience the story first-hand through Iktomi's egocentric first-person point of view as he confronts his environment and establishes his relationships with other characters. Every event revolves around him, and we never see the other characters acting independently of their relationship to him. These results lend additional support for Iktomi's status as the story's protagonist.

Iktomi's egocentrism likely would have stood out for tribal audiences, since his behavior runs counter to their cultural value of placing concern for others before one's own. Iktomi flaunts blatant disregard for the social etiquette of the tribe, and the three different versions consistently represent this theme. Nor does Iktomi make inclusive 
reference to himself as part of any group; he never uses the inclusive you and I or we. The only instance where he uses the inclusive first-person-plural us occurs in Walker's version where he talks to himself as he contemplates how to make use of the Wolf's dead carcass; he muses, "Let's see..." (i.e., "let us see", line 25), which, of course, turns out to be a common figure of speech.

Each version also references Iktomi in the second-person when other characters such as the Ducks engage directly with him in interactive dialogue. However, none of the other characters share a reciprocal relationship with Iktomi, although he seems to perceive them as conscious, rational beings. For instance, he speaks to both the Tree(s) and the Wolf/(ves) and expects an appropriate response from them. In the case of the Tree(s), Iktomi calls out to them imploring them to quiet down; with the Wolf/(ves), Iktomi again calls out to them and tries to ward them away from his roasting ducks.

Occurrences of plural first-person pronouns (we, us) co-reference the Ducks only as a group without singling out any individual, and any time they speak, they do so with a single unified voice. All their actions are choreographed to move in unison as a whole entity, without variation. Hence, when the individual duck falls out of step with the rest, the action immediately draws our attention to the anomalous movement as the asynchronous flow creates a discordant incongruity in the behavior of the group. In tribal culture, the breaking of conformity with protocol and group identification by one of its members held dire consequence for the tribe and could pose serious threat and danger to the wellbeing and continuation of the group. Everyone cooperated to enthusiastically 
discourage disruptive or singular behavior, and immediately put right any such occurrences.

The Ducks dialogue with Iktomi using first- and second-person pronouns in combination, highlighting a level of peer relationship between them on account of their direct communication. Interestingly, personal pronouns other than the neuter it are completely absent with regard to referencing Skiská, who plays the role of spokesman for the Ducks. On one hand, the narrators spotlight the distinct identity of the individual Duck; on the other hand, they downplay his role by failing to engage him directly with the protagonist.

Like the Ducks, the plural Wolves in $\mathrm{Z}$ act as a single unit, and they, too, have their spokesperson in a lead Wolf, which never speaks or acts from individual motivation but only from the standpoint of the group. The lead Wolf's exclusive use of the collective first-person pronouns us and our establishes its identification with the group.

While Iktomi directly addresses the Wolf/(ves) in the second-person, they do not actively engage with him; all their communication transmits through the voice of the lead Wolf which directs all his comments, thoughts, and actions only toward the other Wolves, toward himself, or to the task at hand but never toward Iktomi. In fact, the Wolf/(ves) remain rather elusive; they and Iktomi never make direct personal contact.

In contrast to the Ducks, the Wolf/(ves) act on their own behalf for their own gain and help themselves to the opportunity presented to them. The lone Wolf portrayed in D and $\mathrm{W}$ also acts on his own behalf and responds impersonally to Iktomi's prompts without directly engaging him. Instead, although he sees and hears Iktomi, the Wolf 
mulls over his own thoughts, takes his own counsel, and decides on his own course of action in response to what is put before him without acknowledging Iktomi interactively.

The Wolf/(ves)' and Ducks' use of first-person pronouns establishes those characters in roles the audience can identify with since, through their first-person perspective, we can automatically assimilate their thoughts and actions as if they were our own. The skill of the narrator enables us to insert ourselves into the world of the characters and sense, feel, and think what they do through the power of suggestive narration. Because the first-person references to the Ducks and Wolf/(ves) attributes individual identity to them and creates a sense of peer relationship with Iktomi, the effect conveys an impression of separateness perceived as same-but-different on some level with the main character.

The singular Tree also apparently speaks in Z's version from its own personal perspective in the first-person (cf. text line 14); however, D and $\mathrm{W}$ do not personify the Tree(s) as possessing the same speech capability or awareness. In Z's version, Iktomi's response to the Tree's plea leads to an apparent dialogic exchange, yet, the two-way communication really only exists in Iktomi's imagination, and the Tree does not rejoin him. As a consequence, Z's brief suggestion of consciousness in the Tree(s) reverts to D's and W's inanimate characterization of them. The narration otherwise references the Tree(s) only in the third-person. I will describe Iktomi's special relationship with the Tree(s) at greater length in the next chapter. 
As indicated in Table 10, indefinite pronouns occurring in the texts all fall in the context of general reference to characters other than Iktomi and, in most cases, are used by Iktomi himself when speaking to or about the others. The manner of use of these indefinite pronouns in the texts implies distancing from personal identification with the characters referenced and reinforces the separation between characters. Such nonspecific references downplay the characters' status, whether depriving them of acknowledgement as viably conscious entities or through lumping them together into a homogenous group where individuals do not stand out and get recognized. The effect is most pronounced with the Ducks character in all three versions, reinforcing the narrators' distancing between the male lead character and the feminine aspects that emerge through the Ducks. The few instances where the indefinite pronouns seem to acknowledge the characters in a more personally recognized way also incorporate ambiguous or other minimizing or counteracting effects already discussed which position the characters in their subordinate roles to Iktomi.

\section{Summary of pronoun analysis.}

In summary of the results and findings just presented, the frequency of firstperson pronoun reference for Iktomi far exceeds that of any other character, and he operates almost exclusively in the singular, strongly suggestive that the narrators position his character as the sole initiator of the action. Iktomi stands at the center of attention and everything in the story revolves around him. The events always remain focused on him and his immediate situation, and the other characters do not act independently without direct contact with him. 
Conversely, no other character uses the first-person singular form (cf. the above exception regarding the Tree); the Ducks are almost exclusively referred to collectively and, most often, in the third-person. Despite conversing directly with Iktomi, the Ducks do not operate independently of him, that is, they seem to exist only as far as their direct interaction with him. The narrators treat the Wolf/(ves) collectively in the first-personplural where they are multiple in number (in Z), though in the third-person where solitary (in D and W), and only from the narrator's detached point of view. Thus, portrayal of the Wolf/(ves) deprives them, also, of independent status derived from association with the main character.

Iktomi engages each of the characters separately; his experiences with them do not overlap with other characters, nor do the characters interact at all with other characters. In every version, Iktomi directly addresses the Ducks in conversation with greater frequency than they address him, especially in $\mathrm{Z}$ and $\mathrm{D}$, further emphasizing his role as the protagonist. However, W's version balances the co-reference of secondperson pronoun use between Iktomi and the Ducks and Wolf/(ves) more equally, which hints at that narrator's possible intention to equate all three characters in some manner. The distribution and frequency of occurrence of second-person pronouns in both Z and D tends to place Iktomi on more equal footing with the Wolf/(ves) than with the Ducks, although in Z, Iktomi appears to more actively engage directly with the Ducks than with the Wolf/(ves) — three times more. These leanings refer back to the notion of author preference for certain characters due to self-identification with the perceived sex of a particular character or affiliation with its associated traits. Based on the evidence, I 
suggest that the Ducks and Wolf/(ves) characters represent a certain differentiating facet of Iktomi's own nature and reflect his internal self-conflict as he meets them along each successive stage of his journey.

The narrators also employ the neuter third-person pronoun it in reference to the characters of the Ducks, Skiská, and Wolf/(ves) but never to Iktomi. The neutral reference refers only generally to, either, one unidentified member of the group (i.e., one of the Ducks; Skiská), to the animal form after it has been killed (e.g., duck roast; wolf carcass/pelt), or to the impersonalized character of the lone Wolf in D and W. Because the narrators reference all the characters except Iktomi with the neuter pronoun, they clearly subordinate them to the figure of Iktomi, whom they never impersonalize. It also marks the transformation of these characters from conscious human-like entities to lifeless objects (roast, carcass) or abstract concepts (pelt, wood duck, or Skiská as a named identity). These character forms are unstable and shift out of interactive partnership with Iktomi into the role of inanimate prop.

These observations reinforce my conclusion that each character, in turn, operates as a reflective image of Iktomi's own nature which he must confront and overcome. When Iktomi first comes into contact with another character, he meets it as a figure equal to his own stature. As the encounter unfolds, Iktomi gradually shifts to a more impersonal relationship with the character until, eventually, he has reduced it to a mere object with which he no longer shares any affinity. Once he has worked through the issue the character presents to him, its role and purpose reach fulfillment and conclusion. At that point Iktomi moves on past to the next stage where he will confront yet another 
facet of himself played out by the next character until he has completed his journey. In this manner, each character plays a specific role for a limited extent of involvement; and upon completion of the character's purpose, the narration implies no further interaction with Iktomi and makes no further reference to the character. The lights go out, so-tospeak, on the character's scene in the story.

\section{Documenter Motivation}

In this section, I reintroduce the role of the documenters who produced the three versions of Iktomi used in this study, and speculate about how factors surrounding their background and experience may have been brought into play in the telling of the story. I begin by briefly outlining the links between the documenters and the components of myth narrative. Then I will summarize how the documenters' experiences, background, and attitudes might have helped shape their respective versions.

\section{Documenters' role in the storytelling context.}

In Chapter 2, I put forward the four components of myth narrative as constituting the narrator, audience, context, and situation. Most of my analysis discussed thus far has focused on the latter three components, linking character nature and behavior as delineated by coded traits in the text data to the situation or reasons for telling the Iktomi story in the contexts pertinent to the respective versions studied here. I have also attempted to extract inferences of certain parallels that run between story characters and members of the respective audiences through my examination of the texts. Pertaining to the fourth component of myth narrative, evidence of narrator effect also permeates through the rendered format and storytelling style of the different versions and further 
reveals clues about the nature of each version's targeted audience, the motivation behind the amount of descriptive detail provided, and even the sex of certain participants in the storytelling context. All of these elements are external to the story, that is, they do not exist within the world of the story as the characters and events do, yet they exert influence on the outcome of the telling by creating a backdrop of conditions that give unique shape to the narrative.

The roles of the documenters come directly into play with regard to both the narrator and audience which exist as external participants in the storytelling event. To maintain distinction between the roles of the various participants, I need to clarify some of the terminology that I use in the following discussion. I refer to three speakers, narrator, recounter, and character as follows: the narrator refers to the original storyteller who first related the tale; the recounter is one of the three documenters of the versions - Zitkala S̆a, Deloria, or Walker; the character is a participant that exists within the story world.

In Chapter 2, I indicated that all three versions ultimately originated from Native storytellers or narrators within the Dakota culture. We are told that Deloria and Walker recorded their versions as narrated by male Lakota storytellers, and so, the roles of narrator and documenter or recounter are distinctly separate for those two versions. In the third case, Zitkala S̆a fulfilled both roles since she retold the story she had often heard repeated through her childhood, rendering a fresh version in her own words and from her own perspective, which the other two versions apparently lack. We can assuredly expect Zitkala S̆a's version to feature a large measure of her own personality and style since it 
was common practice for Native storytellers to inject their stories with personal inflection and flavor (Picotte, 1985, xiv). Zitkala S̆a voiced her intentions directly: “ I have tried to transplant the native spirit of these tales — root and all_-into the English language" (Zitkala S̆a, 1901/1985, p. vi). However, although Deloria's and Walker's versions were told by narrators other than themselves (and we have been assured they recorded them faithfully as true to the original), as transcribers and translators recording the narrative, Deloria and Walker still without a doubt filtered the narrator's words through their own frames of reference. Hence, it is virtually impossible to say that neither Deloria nor Walker (nor Keller nor Jahner, who reworked Walker's manuscripts) exerted any effect whatsoever on the ultimate rendering of the story, however minimal.

In addition to narrator effect, Zitkala $\breve{S} a$, Deloria, and Walker also personally bring to bear the effect of audience participation in their respective versions of the story. As cited in Chapter 2, the audience comprises all participants including bystanders and others who may not appear to be involved in the narrative event itself. Since the narrators would censor the text based on all who were in attendance, they would necessarily have included Deloria and Walker for whom their respective texts were narrated. Each narrator would surely have taken into account Deloria's and Walker's presence as representing receptive or interactive individuals as well as their respective backgrounds, experience, and reasons for requesting the story along with any other people we have not been told about who might have been present during the actual telling of the story. Their sheer presence, either through physical or recorded means, could have impacted the ultimate rendering of the tale in a certain way, and this consideration has led 
to my efforts to examine the elements of influence they may have brought to the storytelling event.

\section{Effect of documenter's experience on the rendered story.}

With what we understand about the documenters' experience, we can deduce certain probable suppositions about the rendered forms of the texts. For instance, Zitkala

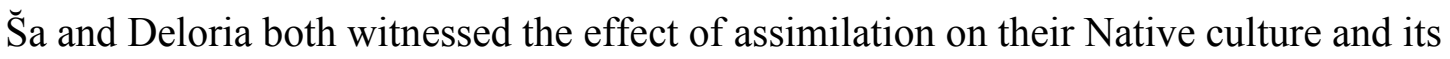
impact on those close to them, infusing both women with empathy and a desire to redeem cultural pride and dignity for their afflicted people whereas Walker observed the impact of oppression and assimilation on the Native people as an outsider without having personally experienced the hardships himself. Although Walker was in a position to sympathize with the Natives since he lived and worked among them, he could not have possessed their personal sense of knowing about their experienced adversity.

Although both the Dakota women were near contemporaries, Zitkala S̆a's and Deloria's experience also diverged; while Zitkala S̆a grew up abused, angry, and separated from her family and cultural roots, Deloria was enfolded within the supportive embrace of her family, church, and community. Deloria enjoyed strong paternal support from the men in her life - the patriarchs of her family who served in community leadership roles and, later, her mentor Boas. Deloria's handling of the story text can be read as reflecting the balance and security she experienced in her life which helped cultivate her careful, systematic attention to evidence while maintaining a professionally neutral attitude toward its content. 
Zitkala S̆a lacked such male parental guidance and nurture and was left to defend her own right to be in the world. Faced with frequent cruelty, Zitkala S̆a lost her childlike innocence at a very early age, and her boarding school experiences inflamed her indignation over the loss of her personal freedom and independence. As a consequence, she emerged combatant and desirous of winning back human rights for the Indian people (Dominguez, 2003, pp. v-vi). As a result of her immersion and education in the White culture, Zitkala S̆a learned how to use the tools of White society such as the written word, the legal system, and political associations to present her arguments and impose her point of view to sway public opinion. Yet, she also understood other subtle and effective means of persuasion exercised through the traditional Native medium of transmitting information in the form of stories. If we look closely at her colorfully descriptive rendition of Iktomi, we may perceive a parallel between the assimilation practices of Western culture and Zitkala S̆a's presentation of the seemingly benign antics of the foolish Iktomi. Much the same way in that the $19^{\text {th }}$ century White oppressors attacked Native culture by luring Native children away from their families and cultural practices, Zitkala S̆a's tale captures the attention of the children of her White audience through the use of vivid color and description of characters and events and garners empathy for the unlucky characters in the story. It is easily possible to discern parallel connections between White invasion and takeover of Indian territories and rights, which, on the one hand, included the killing of innocent and unsuspecting hosts and, on the other hand, involved the humorous activities of Iktomi and his cohorts. Zitkala S̆a's version further implies a moral teaching that shows Iktomi falling short of his intended goal as a result of 
his own treachery, a reflection that ties back to one of Zitkala S̆a's own early experiences when she wished to see her taunters get their come-uppance as she walked away with first prize at an oration contest. In keeping with her life's work, Zitkala S̆a expressed herself as a compelling advocate determined to restore the public image of Native people to a state of autonomy, strength, and self-governance; at the same time, her story conveys a simple moral code of behavior and the corresponding consequences for violating it.

Deloria does not seem to have shared Zitkala S̆a's unhappy experiences growing up; however, because her father and grandfather were so prominent in the Native community, continuous stories of hardship and suffering would surely have permeated the daily life of their family as well. Deloria's compassionate nature likely grew out of that environment, while her formal education also equipped her with the skills to effectively promote awareness, understanding, and compassion for her people. To my knowledge, Deloria did not experience the intense anger, betrayal, and abandonment Zitkala S̆a felt. Instead, Deloria acquired respect for learning and cooperative collaboration to effect social change, due in large part most likely, to her association with the anthropological team at Columbia University. Consequently, both her Christian and educational experiences fostered an approach to social change through systematic collection of information and data, examination, and presentation of results and findings. Unlike Zitkala S̆a, who detested her teachers and authorities, Deloria deeply respected and cared for her associates and cooperated with them to help enlighten and inform others about her culture. Her version of Iktomi reflects her diligent, controlled approach in presenting the text as true to that provided by her informant without imposing her 
perspective on the data, while containing her concern for wider understanding in added explanatory footnotes. Her informant would probably have recognized Deloria as culturally savvy although, also, as someone who would share the story with academics; and so, in the guise of proficient storyteller, he would have filled in the tale with enough information for the text to be understood in a general sense by cultural outsiders as well as by those who would have possessed a basic working knowledge of Dakota cultural values and practices.

As women, Zitkala S̆a and Deloria did not overtly dwell on the masculine themes or single out specifically male-oriented activities in their versions, although certain elements pointing to male behavior are present in both. Some of these indicators are possibly results of cultural conditioning proving transparent to the women as cultural insiders so that they may have simply overlooked them. Another reason for downplaying a male theme is that the issue was not particularly relevant to either Zitkala S̆a's or Deloria's purpose. Like Zitkala $\breve{S}$, Deloria's informants would have understood the hopelessness of the Indian people now confined to reservations. In such regard, they may have harbored a desire to provide hope and encouragement to their people while instilling in them a sense of dignified sophistication heretofore unrecognized by non-Indians in addition to preserving cultural knowledge through the traditional means of storytelling. Deloria's informants may have further recognized the potential of her connection with the White educational institution as a means to preserve Native cultural heritage, so it is also possible they may have encoded more of the veiled significance of the story as a structural framework for their ritual practices, as I have claimed. 
Walker presents an interesting situation; although he was a White man and outsider to Dakota culture, his version turned up the least descriptive information of the three texts, rendering it quite difficult for the casual reader to comprehend or find entertaining. More expectedly, greater detail would have been required to fill in the gaps of information for one unfamiliar with the culture. I account for this anomaly in two ways. First, Walker had lived among the Lakotas on Pine Ridge for the 18 years during which he collected stories, and, in the process, he learned some of the language and cultural practices over that time. Several of his letters to Clark Wissler, particularly from 1908 to 1911 , contain updates on the progress he made in understanding Lakota culture and language as he collected information from his informants (Walker et al., 1983, pp. 210). The Lakota medicine men had also initiated him into their ways of secret knowledge, which included the oversight of traditional practices and rituals. They did so in part because they sought to enlist Walker's help in documenting and preserving their practices. As a result, Walker gained a vast amount of insight into the intricacies of Dakota culture and values from their perspective. Because the elder men had already provided Walker with a background of understanding at a very deep level during his training, they would have no need to further spell out the details of their instruction to him in the story. Second, as a male contemporary of his mentors, Walker would have been privy to talk among the men. As with any speech community where participants share membership, ellipsis commonly occurred and did not detract from members' comprehension of the conversation. That Walker wrote his notes and filed them away without immediately reworking them may be due to time constraints; he was a busy man 
as agency physician tending to the sick, doing his own research on tuberculosis, and taking guidance and instruction from the leaders of the Indian community. Further, Walker's informants were also storytellers, so in the spirit of their craft, they required only the essential ingredients of the plot to recreate the episodes according to a future context, situation, and audience, taking creative liberty to embellish their tale as needed. The point is that Walker and his informants seemed to agree that in the expediency of time, only the most important points were necessary to capture the information required to reconstitute the story from its component elements, and the result yielded a skeletal framework of plot and characters with gaps in information that may have come up separately at an earlier time in a different context or conversation among the men. These inferences thus support the notion of a built-in framework for a ritual for tribal males.

From another point of view, some of the variation found among the texts may also be attributed to the different storytelling styles used by women and men; Muchnik and Stavans's (2009) study on storytelling interactions between mothers and fathers and their children in western culture showed that storytelling style depended on the gender of both the speaker and the addressee. The study indicated that mothers tend to use stories "to reinforce their emotional experience," whereas fathers do not employ emotional elements with either their sons or their daughters (p. 68). This non-emotional approach might be reflected in Walker's and Deloria's versions of the story, which were both narrated by men. However, as the sole woman storyteller writing for an unknown audience, Zitkala $\breve{S}$ a fashioned her story for an audience she perceived only in her mind, and she could not 
have known the sex of her future readers; yet, her version, which she wrote with childlike appeal, reflects an emotionally-charged style of elocution characteristic of women.

\section{Case for author preference for Ducks and Wolf/(ves) characters.}

Earlier in this chapter, I suggested, based on the Polarity-Code Data Analysis, that Zitkala S̆a may have favored the Ducks over the Wolf/(ves) and that Walker may have favored the Wolf/(ves) over the Ducks with regard to their relative importance to the storyline of Iktomi. Now I would like to explain my reasoning and conclusion to provide additional support for my interpretation of the results. Much of the information I give here derives from my personal experience practicing Dakota and other tribal customs and traditions. Some of the information stands as general knowledge in Dakota culture, and some I have gleaned over the years from listening to elders and ceremonial leaders in casual settings or during participation in certain rites. I also note that, traditionally, tribal peoples would observe the behaviors and habits of animals and birds in the wild in order to understand their power and influence and they would liken their qualities to human experience. In other words, by studying the wild creatures and learning their characteristics and behavior, people could apply their animal "wisdom" and emulate the animals' example to improve their own ways of living in harmony in the world.

There is possibly a link between Zitkala S̆a's sex/gender and her apparent preference for the Ducks, and her status as female reaffirms the presence of feminine attributes of the Ducks' character. The Ducks' connection to the water, collective enterprise, curiosity, and emotionalism link them to archetypal and stereotypical feminine tendencies in the Dakota worldview regardless of the fact that all the versions use the 
masculine pronoun in English translation to reference the singled-out Duck. Two versions of the story clearly portray the Ducks as a mixed group of males and females: Zitkala S̆a's description of the Ducks' performance strongly suggests a social dance such as a round dance, since they hop continuously in a circle while bobbing up and down. Social dances of this nature were performed by both sexes together as a means for mixing, courting, and engaging in mixed society in a socially acceptable way; hence the implied inclusion of both males and females. Deloria highlights her assumption of male Ducks in line 9 of her story text, noting specifically and as an afterthought, that the females were included in the dancing. This single mention is Deloria's only reference to the presence of female Ducks and stands out on account of its odd placement, whereby it draws specific attention to the character's sex. Walker makes no suggestive reference to any of the Ducks as being female; he confirms their male-only status via their initiating address to Iktomi as they call out to their "Older Brother."

Working with the assumption that Zitkala S̆a's version incorporates Ducks of both sexes, I uncovered definite clues that then indicate segregation between male and female activities among the story Ducks and reflect cultural expectations. For instance, in Dakota culture — during earlier times, at least_ only male members of the tribe engaged in certain activities that the Ducks in the story evidently take for granted. For example, propriety dictated that women would not normally speak to an older male without his first addressing her, and she certainly would not use his given name; nor would a female ask what a man carried in his bundle. The Ducks in the story flout these taboos in, both, calling out to "Iktomi" or "Ikto" and demanding to know what he carries in his pack. 
Zitkala S̆a further depicts the Ducks singing and drumming as they sit together around a drum large enough to include everyone in their circle; these activities were reserved for the men. Dakota women did not beat the drum in this type of setting and would join in singing only while standing behind the seated men. The male Ducks also directly confront Iktomi; alert to the approach of a stranger, they jump up and encircle him, demanding to know what he is about, akin to a direct challenge by warriors protecting their encampment. Finally, Iktomi prepares a sweat lodge-a small domed structure made of willow sticks, reeds, and grasses — for him and the ducks prior to beginning to dance to his special song. The lodge, dance, and song are all indicative of a ceremony; Dakotas used the sweat lodge in a purification rite preceding all important ceremonies or endeavors, and usually only men undertook the rite. Further, men and women did not ordinarily sit together in the same sweat lodge except in a case involving a close-knit family undertaking.

As males, Walker and/or his informant could possibly have identified more closely with the Wolf/(ves) which, in the story, stereotypically exemplify masculine characteristics. Cultural emphasis would likely have predisposed Walker and his informants as men to identify with the parallels between the behavior of the ideal male and these Wolf/(ves). As leaders, providers, and protectors of their families, Dakota men strove to mirror the natural wolf's example. The Dakota revered the wolf's intelligence and hunting skill and emulated its practice of sharing its provisions with others before partaking of anything that might be left after all others had availed themselves. Tribal people also looked to the wolf as teacher and guardian of the family; as such, the 
Wolf/(ves) in the story demonstrate the social values of good manners, etiquette, and concern for the welfare of others. The Wolf/(ves) respond to Iktomi's call and inadvertent invitation, and they accept his proffered food, including additional helpings. In Dakota society, one did not refuse food, as to do so would give offense. Thus, the Wolf/(ves) eat with gusto so as to compliment their host. Conversely, social norms dictated that hosts always shared whatever they had without holding back; thus, Iktomi's stinginess over the roasts stands out as an affront to the social norm

The fact that Deloria showed no preference for either Ducks or Wolf/(ves) may be accounted for in remembering that she strove to write down the story as she recalled its narration (although, she did so sometime after the storytelling event took place). As a result, she might likely not have embellished the details but would have recorded the story to the best of her ability to remember the way the narrator related it. The interesting point to make holds that each version presented Iktomi, the Wolf/(ves), and the predominant figure of the Ducks characters as male, which clearly reflects cultural norms with respect to the male-oriented activities, discourse, and interaction among the characters. At the same time, each of the characters also exhibits certain feminine characteristics to a greater or lesser extent, and I contend we may discern the presence of these characteristics, in part, through the subtle emphasis exerted and influenced by the sex of the documenter.

In the next chapter I will present my interpretation of the findings as they relate to my thesis that Iktomi encodes a framework for the Dakota vision quest. 


\section{Chapter 5: Interpretation and Discussion}

In this final chapter, I summarize my interpretation of the elements of the story which I found to support my claim that Iktomi functions as a Dakota vision quest narrative. I illustrate and explain some of the symbolism, metaphor, and physical and iconic aspects of the characters I alluded to in Chapter 4 as well as how the relationships mesh between characters and support the development of a story plot that corresponds to the Dakota vision quest framework. Along with these points, I will summarize the emergent themes of male focus, human-like representation of the characters, and the issue of duality in the human experience. I begin the chapter with an explanatory overview of the Dakota vision quest rite to help the reader follow my subsequent interpretation of the text.

\section{Overview of the Dakota Vision Quest.}

To lay the foundation to substantiate and defend my claim that the Iktomi story serves as an encoded outline of the Dakota vision quest, I present the reader with a basic description of what the vision quest involves. I offer only a brief sketch here and will elaborate in greater detail later in the chapter, when I will link aspects of the rite with elements I found in the story. I derive my descriptions of the vision quest, in part, from various sources, including Walker's Lakota Belief and Ritual (Walker, DeMallie, \& Jahner, 1980, pp. 78-80, 129-135), teachings I received from various Native American elders and medicine persons, and my own experience through participation in the sacred rites of the Dakota people. 
The Dakota hanbdečeyapi (Lakota: hanblečeyapi), or vision quest, is one of the seven sacred rites of the Dakota Sioux tribe. Among other reasons, adolescent males would undergo the vision quest as a rite of passage marking their transition from child to adult, upon which, they would begin to assume the responsibilities of men as protectors and providers for their people.

For the vision quest, which is still practiced today, the seeker would go off to a secluded site, usually on the top of a hill, where he would remain alone to pray and commune with the spirit world, usually for up to four or five days and nights, to obtain guidance and direction for his life. Although his experience remained a personal and private individual endeavor, the information he brought back was intended to be shared with the people to help strengthen the society.

While on the quest, the seeker had to endure a fast without food or water for the entire time during which he underwent a process of physical, emotional, and spiritual self-confrontation. Along with increasing physical discomfort, various emotional issues would surface through a series of stages, and he had to wrestle with each one in turn to regain a state of inner peace and balance. If and when he successfully achieved his state of equilibrium, he would receive his guidance. If he was unable to overcome his emotional turmoil, then that, too, served as a message, directing him to go on working to improve his life and relationship to the society and the natural environment the group inhabited. Perhaps then, at a later date, the seeker might attempt another fast when his mentors deemed him more appropriately prepared. 


\section{Stages of the Vision Quest.}

The vision quest normally involved a series of stages through which the seeker would move to come to terms with his purpose. In the first stage, the seeker settles into his seclusion and begins to pray in earnest, first, for the welfare of his relatives and, only secondarily, for help for himself. His selflessness in praying for others and offering his sacrifice of going on the vision quest, along with his careful and meticulous preparation, envelop him with a sense of satisfaction and pride. He begins to feel open and at one with the sacredness of all things, pleased with himself for undertaking such a fine endeavor for the sake of his people. In short, he is full of himself.

By the second stage, the seeker has run out of people and things to pray about, and he gradually begins to grow weary of waiting for the guidance to come. Little by little, he starts to notice the sounds and sights around him, and often, he will grow dismayed to find out he has settled himself in the midst of some irritant such as an anthill full of crawling, stinging ants, biting insects, or other unfortunate circumstance that he did not notice when he selected his site. The nagging annoyances remind him he is a flawed being and uninvited guest in their domain, and he must struggle to regain balance and acceptance of his insignificance against the greater powers and natural forces of the universe. Naturally, in due course, frustration, irritation, and impatience enter in as his companions. As he labors to center himself again and regain peace, he reminds himself that all living creatures are his relatives, and he attempts to access his more altruistic nature and thus elevate himself in the eyes of the spiritual forces whose help he seeks. 
But the more he strives toward effulgence and control over his impulses, the more the persistent irritation increases. He may first try to talk to the agitators and appeal to them as his kin, as all things are related in the Dakota worldview; however, realizing his words effect no change, he then devises schemes to redirect their movement away from him so he can still feel harmonious with them. Yet, as nature inevitably refuses to adapt itself to his wishes, the seeker must eventually drop all pretenses and accept his powerlessness, at which point he must choose a course of action: he must surrender to his predicament or leave.

\section{Analysis of the Vision Quest and Iktomi's Journey.}

In accordance with the purpose and objective of storytelling in tribal culture, I conclude that Iktomi, as the central character, stands as a signifier for each individual in the audience, while the Ducks and Wolf/(ves) represent aspects of the individual's own nature. The story acts both as a moral parable imparting a message of instruction and/or caution to the listeners and as an instructional guide to prepare youths for the eventuality of the rite of passage into adult responsibility in the form of a vision quest. Whether embarking on the very personal inner journey toward self-improvement via selfcontemplation or undertaking an actual vision quest, the seeker must encounter and contemplate each aspect of his or her own character in turn and work through any internal conflicts or issues that surface through contact with their own basic nature. The story characters reflect the issues, conflicts, and natural tendencies confronting the individual through their characterizations and the dynamics of their interactions. In particular, 
Iktomi conducts himself antisocially throughout the story, flouting the conventions of normally accepted behavior and attitudes that the culturally initiated will quickly grasp.

As a side note, I acknowledge that in today's world, both males and females take part in the vision quest ceremony; however, in the historical context of when these versions were collected, women rarely did so. Therefore, for ease of communicating the essential points of information and because I affirm the story's focus on male activity in the society, I will assume an implied male connotation in the following discussion by referring to the seeker only in the masculine. In doing so, I do not imply that the possibility of female participation did not exist.

Returning now to the analysis, Iktomi's encounters with other characters are exclusive; he engages with them one-on-one so their interactions are contained and discrete without overlap. Zitkala S̆a walks Iktomi through three such separate encounters — with the Ducks, Tree(s), and the Wolf/(ves); Deloria and Walker take him through a fourth encounter via a second meeting with the Wolf/(ves). The important detail with regard to the Iktomi story lies in the consistent arrangement of consecutive scenes across the different versions, which supports the notion of a centralized basic structure of the plot, and by analogy, of the vision quest ceremony.

The sequence of Ducks-Tree(s)-Wolf/(ves) proves essential for describing the unfolding nature of a vision quest. The seeker and Iktomi, in mutual representation, embark on their journey in a state of enthusiastic optimism and enter the first phase, represented in the story by the encounter with the Ducks. Having found a secluded location (often on a hilltop), the seeker clears and marks off a space large enough for him 
to only stand or lie down. He will create an enclosure around the space where he must stay, and he cannot leave until he completes his quest. (Zitkala S̆a and Walker indicate such a hill in line 1 of the text, which Iktomi approaches after wandering aimlessly for an undetermined time.)

The seeker steps into the space at the south side to begin his ordeal, and he begins his prayers, appealing to the powers in the western direction. Zitkala S̆a's version depicts Iktomi looking toward the "western sun," a distinct reference to the starting direction of a vision quest (cf. text line 2). As he gazes westward, he espies the group of Ducks, which correspond to the Dakota guardian of the South, who "is the giver of life" and whose messengers are the water fowls (Walker, DeMallie, Jahner, \& CHS, 1991, p. 127). In this role, the Ducks or water fowls serve as overseers of customary tribal practices while maintaining social order.

This first phase of the vision quest involves the meeting place of unlimited potential and abundance where there exists no judgment of good or bad but only of possibility. For the Dakota, flocking ducks signified beginnings such as the start of a new year or new cycle (Walker \& DeMallie, 1982, p. 123), and they also connoted generosity and hospitality (p. 106). The Ducks in the story invite Iktomi to join them in a spirit of goodwill, enjoyment, inclusiveness, and contentment, and they represent peaceful harmony and emotional buoyancy in a passive, non-resistant, and agreeable style.

At this stage of their journey, Iktomi and the seeker contemplate their need or purpose and make their appeal in peaceful non-resistance to the environmental conditions 
while holding confident expectation that they will obtain what they hope to achieve on their quest. The vision seeker uses this phase to offer prayers to the powers of the spirit world and to the four cardinal directions. He selflessly pleads help for others in need and to state his question or reason for requesting guidance.

By and by, a mild struggle flares up to threaten peace and security: in the story, the Ducks discover Iktomi's treachery and danger to their welfare; for the individual on quest, the threat materializes in the form of personal shortcomings that come to mind reflecting the seeker's own flawed character. In both scenarios, the disturbance arises from the revelation that the actual state of the situation is not what it had seemed. The Ducks Iktomi has killed symbolize the seeker's suppression of the negative emotional undercurrent that jeopardizes his spiritual purity, while those which escape represent the seeker's flight from confrontation with his source of contention. Jahner (1983) suggested that "narrators and audiences in pre-reservation times were accustomed to the idea of fleeing various kinds of situations in order to find (or regain) a particular state of mind or well-being that, to them, meant home" [italics in original] (p. 175). Iktomi and the seeker conclude this phase in a wash of smug self-satisfaction, pleased that they have successfully prevailed over the situation. The ducks flee to safety, while Iktomi moves to a place of rest to prepare his feast, well satisfied with his work.

The next stage slowly overtakes Iktomi and the seeker as they calmly anticipate the results of their labor. However, the waiting becomes tedious and they have nothing to do, yet, they cannot leave the situation. Iktomi must stay and guard his ducks which he has set to roasting, while the seeker remains confined to his secluded enclosure. They 
grow acutely aware of myriad sights and sounds all around, and eventually a persistent bother begins to irk them.

In the story, the annoyance takes the form of two trees or two tree branches rubbing together to make a persistent squeaking noise. The Tree(s) convey a sense of standing upright and, for the seeker, connote moral and ethical conduct. The Tree(s) do not just stand there inert but are active and alive (E. C. Deloria, 1998, p. 53): they support, nurture, and provide nourishment, fuel, shade, and shelter. Many cultures have regarded trees as sacred symbols, such as the Tree of Knowledge of Good and Evil and the Tree of Life in the Judeo-Christian tradition. The Tree(s) in Iktomi stand as a comparable symbol, rooted in the earth while reaching toward the heavens, thus serving as a link between physical existence and the realm of spirit. Upward climb signifies lifting oneself up out of the material world to a more pure state of harmony and unity with all of Creation. The Dakota people have their sacred tree in the cottonwood, whose forked branches figure prominently in their cosmology as a symbol of man's duality and the two paths of morality or social deviance that one may choose to follow. Accordingly, the squeaking Tree(s) in the story represent the tension intrinsic to that choice.

Remembering they are on a sacred mission, both Iktomi and the seeker first respond to the nuisance that arises through their attempt to harmonize and appeal to its sense of sacred relatedness. However, Iktomi's and the seeker's aggravation increases when they fail to exert their control over the natural forces and align the irritant to their own will. They need to find relief and take action, yet, in their attempts to further control the circumstances, they only exacerbate their situation: Iktomi finds himself caught in the 
Tree(s)' branches, whereas the seeker may exhaust himself through fret and worry or perhaps increase his physical discomfort through scratching or entangling himself in his gear and paraphernalia.

Realizing the futility of their struggle, Iktomi and the seeker break down to experience the baser emotions of self-pity, fear, and anger. Here, they hang in the balance between the material world and the world of spirit, and here is where they meet the opportunity to effect a change in their condition. Rendered helpless and vulnerable, they are poised for an opportunity to overcome their baser passions, or they may choose to grovel in their own misery, feeling sorry for themselves and imagining how the world has treated them so unfairly. From his perch high in the Tree(s), Iktomi has unwittingly elevated himself through his ordeal and acquires a clearer view with far-reaching vision. Meanwhile, the seeker will also hopefully rise up to a higher level of awareness with the realization that he struggles in vain and accept his predicament.

Caught fast in their dilemma, Iktomi and the seeker enter the third phase of their journey when they perceive a new potential threat approaching from the distant reaches of their imaginations. Once again, baser instincts overtake them as they react with anticipation of impending danger and conflict. In the story, the contentious element arrives in the guise of Wolf/(ves) coming to steal Iktomi's cache of food; for the seeker it may take the form of any spirit animal or being that manifests to bring him a spiritual message.

The Wolf/(ves) or spirit guide come as the Great Teacher, bringing knowledge and wisdom for those ready and willing to receive it. In tribal society, wolves 
symbolized speed, craftiness, invisibility, and success in war (Walker \& DeMallie, 1982, p. 96). They could also assist a hunter in locating game (Densmore, 1992, pp. 179, 183). In this capacity, a guide in the form of a wolf would bring the Dakota vision-quester faceto-face with the state of his hunting prowess, tactical skills, patience, and bravery as well as the reality of his readiness to protect and provide for his people.

Fear overcomes Iktomi, who projects his own negativity onto the passing Wolf/(ves). He is unable to control his trepidation and remain calm and watchful, and his agitation discloses his location, which draws the Wolf/(ves) closer. Iktomi inadvertently shows himself to be inept as a warrior and protector of the people by revealing his underdeveloped sense of responsibility for the tribe. Compounding the sham of his pretense, Iktomi's ungracious and stingy display of antagonism toward the Wolf/(ves) further exposes his contempt for the social values of generosity and kindness as he attempts to withhold sharing his food.

The parallel for the seeker unfolds through the manner in which he interacts with the spiritual messenger who has appeared to him. If he reacts with courage and selfrestraint, he may receive a message of guidance; the danger will pass and the seeker will be able to return to his normal state and enjoy the benefits of his effort. However, should the seeker respond in accordance with Iktomi's behavior, he could suffer additional hardship or possible injury in the case that he mishandles a situation with a dangerous animal or malevolent spirit. Such an unhappy result would indicate the individual has not developed the maturity and self-discipline to allow him to participate in adult activities, a deficiency which could prove harming to the safety and welfare of others. 
Another clue to support the analogy between the Iktomi story and the Dakota vision quest resides in Iktomi's failure to partake of food on his adventure despite his attempt to do so. The story serves two functions here which point to the vision quest: because the whole crux of the tale lies in Iktomi's quest to secure a meal, it highlights a cultural faux pas by mocking it, e.g., a seeker is not supposed to eat or drink while on a vision quest; and the difficulty Iktomi experiences over his hunger pangs forewarns the seeker not to give in to temptations while in seclusion on the quest lest undesired results ensue. The seeker must fast for the duration of the quest even though he may suffer greatly from thirst and hunger, a state that coincides with Iktomi's enterprise. Iktomi's first temptation looms in the form of ducks swimming in water, while the seeker's hunger and thirst evoke similar initial emotional triggers that must be wrestled with. Like Iktomi, the seeker may find his thoughts clouded by these very physical discomforts until he is able to refocus his mind toward his loftier purpose (cf. the symbol of the tree). He reorients himself through rest and contemplation until ready to resume his spiritual quest.

The result of Iktomi's, and correspondingly, the seeker's pride and selfishness on his quest culminates in failure to procure satisfactory results (i.e., obtain food/receive a vision), and Iktomi sulkily goes on his way without having learned anything. In Deloria's and Walker's versions of the story, the narrators continue Iktomi's escapade through a fourth phase which reveals what lies on the road ahead. Iktomi comes upon the cause of his downfall, finding the Wolf lying presumably dead. The immobile form of the Wolf represents latent knowledge and understanding lying unused and discarded. One more time, Iktomi fails to recognize and understand its value and he curses the carcass and 
kicks it around. However, unable to find a use for it, he eventually tosses it away in frustration. The seeker may potentially follow Iktomi's course; rather than learning a valuable lesson from the clash with his spirit guide, he may renew the struggle and blame the symptom for causing his own failing so that, once more, he reinforces the thread of negativity and social nonconformity that rule his life.

Although I eliminated the Wind and Fire as characters, their roles as elementals in the story merit brief mention. Wind and Fire represent the essence respectively of spiritual power that flows in and through all things and transformative power. Their presence signals change and transformation, and when they appear in the story, they alert the listener/reader to a turning point in conditions. As the all-powerful breath of the Great Spirit, the Wind acts to remind the seeker that a more powerful force dominates his life and he is not the one in control. It is also the Wind that whispers one's own fears, permeating one's mind with real or imagined thoughts and ideas, and the caution for the seeker is to listen carefully to his inner promptings and use discernment to make thoughtful, sound decisions.

Fire effects changed conditions and also manifests with dual purpose in the story as well as for the vision quester. It can be used constructively, such as for cooking, or it can consume and destroy those who are not careful with it. In the story, Iktomi builds a fire in preparation for each step of his activity; there it takes on a purifying function, for example, as with the fire for the purification sweat lodge in Zitkala S̆a's version. Iktomi also uses Fire in his attempt to destroy the Wolf once and for all; in Deloria's version, he tosses the carcass into the Fire, while, in Walker's version, Iktomi chases the Wolf 
around the embers. The seeker uses the fire symbolism in his purification rite to prepare for his vision quest, but Fire can also represent the flames of his own passions that he must wrestle with while on the quest.

The story therefore illustrates that only through sincere effort to recognize, contemplate, and overcome his flaws will the seeker establish himself as a reliable adult member of society on whom others can depend. In order to achieve the successful goal of the vision quest, he must (1) experience and move through the emotional stages of anticipation-frustration-fear-anger, (2) surrender to forces greater than himself, (3) recognize his true relationship and responsibility to other living beings, and (4) develop the maturity to accept himself with courage, truth, and honesty. The character Iktomi demonstrates that, if an individual should follow his example and fail to own up to such challenges, he will not attain the respect of his fellows or be able to live harmoniously among his social milieu.

\section{Emergent themes.}

\section{Male focus of story.}

In Chapter 2, I noted that the trickster Iktomi is always portrayed as male in the three versions rendered by Zitkala S̆a, Deloria, and Walker. The data have clearly indicated that this choice of character sex was not arbitrary; on the contrary, the narrators' choice was intentional and carries great significance for the purpose of the story. Horne (1999) spoke of the trickster's mirroring aspect which allows for both male and female (pp. 129-130) representation. Furthermore, although male characterization is frequently encountered in mythical stories of the Dakota people, it is not an exclusive 
standard in their ohuykakay tales. Other stories in this classification feature female characters and female protagonists such as "Meadowlark and the Rattlesnake," "The Rolling Skull,” and “A Woman Kills Her Daughter," all from Deloria's (1932) collection in Dakota Texts. Similarly to Iktomi, a surface reading of these female-oriented tales imparts teachings of moral values that would be relevant to women and girls in tribal society. And again, just as with Iktomi, a more in-depth and systematic analysis such as I have undertaken in this study would be necessary to elucidate the possibility of another veiled construct that might serve as a framework for a specific cultural practice that may be pertinent to females..

The evidence I have given through analysis of this story presents Iktomi as a male entity that Dakota males can readily relate to; for example, he goes out alone, hunts, and runs ceremonies, none of which were normal practices of Dakota females. Analogously, a Dakota male would embark on the vision quest as a rite of passage into adulthood where he would be tested and challenged to throw off childhood behaviors and attitudes and begin to put the welfare of his people before his own needs. The solitary ordeal of the vision quest would prepare and initiate the young man into the role of responsible and reliable protector and provider of the tribe.

\section{Human-like characterization.}

The human-like qualities ascribed to each of the characters also play into audience member recognition, and I have shown that each character represents an aspect of human behavior, thought, or attitude that audience members can personally identify with, while the activities the characters engage in are specific to one sex or another. While Iktomi 
was himself depicted as male, the Dakota yet recognized that every individual carries aspects of both masculine and feminine character. These aspects emerge through representation of the Wolf/(ves) and Ducks characters, respectively, and I described their manifestation in detail in Chapter 4. Because both masculine and feminine attributes are present and recognizable through the characters, all audience members have the opportunity to see themselves somewhere in the story, regardless of their own sex. In that way, the story still carries appeal for female audiences and doubles as a moral parable in addition to its template purpose for the male-oriented vision quest.

\section{Duality in human experience.}

Another persistent and parallel theme in the story and in life emerges as the nature of duality present in humans. The tale is steeped in the theme of duality which the Tree(s) explicitly symbolize in tangible form, whether as two separate trees or two branches of the same tree, depending on the version. I discussed the significance of the forked cottonwood tree for the Dakota earlier in this chapter; however, duality prevails through additional numerous contrasts throughout the story such as masculine versus feminine aspect, choice of right or wrong action, individual versus group focus, raw or cooked state, and so forth. Each contrast represents a point of decision for a character in the story as well as for the vision-quester working through his emotional state of mind and spiritual development. As an instructional guide to morality and ethics, the duality in the story provides the audience members myriad questions to ponder in examining their own behaviors and standing in the community. Likewise, for the vision-quester, the duality presents not only an exercise in moral judgment but also the opportunity to refine 
critical skills of judgment and decision-making required for survival and to be a skillful and successful hunter and warrior as well.

\section{Summary and Conclusion}

At the beginning of Chapter 4, I claimed that my analysis revealed aspects of the Iktomi story which affirm key points that support my thesis, and I elaborate on that assertion with a synopsis of the supporting evidence represented in Table 11.

Table 11.

Summary of Character Roles, Relationships, and Correspondence to Vision Quest

\begin{tabular}{|c|c|c|c|}
\hline Character & $\begin{array}{l}\text { Character } \\
\text { Roles }\end{array}$ & $\begin{array}{c}\text { Character } \\
\text { Relationships }\end{array}$ & $\begin{array}{c}\text { Character correspondences } \\
\text { with Vision Quest }\end{array}$ \\
\hline Iktomi & Protagonist & $\begin{array}{l}\text { Central focus of story } \\
\text { events; identifies } \\
\text { strongly with } \\
\text { masculine principle } \\
\text { (proactive, rationale) }\end{array}$ & Seeker on Vision Quest \\
\hline Ducks & $\begin{array}{l}\text { Mirror of } \\
\text { Iktomi's } \\
\text { feminine aspects }\end{array}$ & $\begin{array}{l}\text { Identify with feminine } \\
\text { principle (reactive, } \\
\text { emotional, distanced } \\
\text { from protagonist) }\end{array}$ & $\begin{array}{l}\text { Encounter with emotions: } \\
\text { positive/negative }\end{array}$ \\
\hline Wolf/(ves) & $\begin{array}{l}\text { Mirror of } \\
\text { Iktomi's } \\
\text { masculine } \\
\text { aspects }\end{array}$ & $\begin{array}{l}\text { Identify strongly with } \\
\text { masculine principle } \\
\text { (proactive, rationale, } \\
\text { aligned with } \\
\text { protagonist) }\end{array}$ & $\begin{array}{l}\text { Encounter with fear; } \\
\text { responsibility }\end{array}$ \\
\hline $\begin{array}{l}\text { Skiská } \\
\text { (Lead Wolf) }\end{array}$ & $\begin{array}{l}\text { "Spokesperson" } \\
\text { for respective } \\
\text { group }\end{array}$ & $\begin{array}{l}\text { Give personal } \\
\text { expression to } \\
\text { feminine (masculine) } \\
\text { aspects }\end{array}$ & Personal response to situation \\
\hline Tree(s) & $\begin{array}{l}\text { Duality and } \\
\text { choice }\end{array}$ & $\begin{array}{l}\text { Nexus of conflict with } \\
\text { dual nature }\end{array}$ & $\begin{array}{l}\text { Choice; spiritual } \\
\text { upliftment/enlightenment }\end{array}$ \\
\hline
\end{tabular}


Four of the five story characters - Iktomi, Ducks, Wolf/(ves), and Skiská—coidentify, meaning that they share aspects that link them to one another in close relationship. As shown in the table, each character plays a unique role that places that character in a particular relationship with the protagonist Iktomi. Additionally, each character has been shown to correspond to the progressive stages experienced by the seeker on vision quest. While the Tree(s) do not identify with Iktomi per se, they do represent a critical phase of his journey as well as the pivotal point of decision for the vision quester. The tabulated information shows a consistent thread of representation of the characters and their roles and relationships between the story and the vision quest experience. Relative distance between characters is also observable through the relationships indicated and reinforces the conclusions I have drawn regarding the characters' function in the story and their significance pertaining to the vision quest ritual. I summarize additional evidential support for the Iktomi story as a Dakota vision quest template as follows:

$\sim$ The story is essentially constant in content and form; All three versions incorporate consistent structure and essential elements of the tale;

The story's cyclical episodes correspond to the progressive stages of the vision quest experience;

$\sim$ The protagonist Iktomi engages in non-overlapping, sequential, and discrete encounters with each of the other characters, representing the experiential stages of the vision quest;

All character activity revolves around the protagonist Iktomi (i.e., vision quester);

The characters reflect differentiated aspects of the protagonist Iktomi (i.e., vision quester); 
$\sim$ The characters' relationships signify themes that relate to self-contemplation, a major feature of the vision quester's experience;

$\sim$ The story's pervasive theme of duality reflects the seeker's moral responsibility and choice which is attained or reaffirmed through self-confrontation;

$\sim$ The story orients toward a male focus which points to a Dakota male rite of passage.

My analysis of the texts of the three versions of Iktomi has revealed that the story serves an inherent purpose of encoding, preserving, and transmitting cultural information across generations and cultural boundaries. I maintain that this story particularly encodes the skeletal framework for the male rite of passage into adulthood, that is, the Dakota vision quest. I have demonstrated through character traits analysis, polarity-code correspondences, and analysis of pronoun usage that the elements of the vision quest can be discerned through the representation of the story characters, relationships between characters, and the activities and interactions they engage in. I have also shown the relationship of the story characters linked to the four components of the storytelling event — narrator, audience, context, and situation — and how the stories have also been used to impart teachings of morality and right conduct among tribal members for individual self-improvement and to maintain healthy social order. Evidence in the data has indicated a male focus inherent in this Iktomi story and is supported by my examination of the role and influence of the documenters who recorded and translated the texts. I further identified emergent themes that correspond to the Dakota worldview and tribal approach to nature and the environment. And finally, I have demonstrated the applicability of the stories to different circumstances and conditions, showing the stories 
to be universal and adaptable across time and space and usable to all, regardless of background, age, language, or experience.

In conclusion, I acknowledge that this study demonstrates the great importance cultural stories hold for preserving and revitalizing Native American tribal practices, customs, ceremonies, and language. The analytical tools presented herein have revealed new insights into Dakota culture while introducing a fresh and innovative approach to rediscovering and reviving endangered cultures. The ability to extract hidden information from documented text informs our understanding of the culture with a practical perspective. In turn, this new way of understanding tribal practices facilitates derivation of intended meaning in the language of the text, particularly for versions rendered in the Native language. If we can understand the message carried in the text, we have a better chance of extrapolating the meaning of the language that conveys it. Additionally, this method can be used to assist in authenticating different versions of stories as one and the same tale as well as to reveal clues about the circumstances surrounding the storytelling event itself and for whom the story may be relevant. Further study in applying this method to other Dakota tales is required in order to ascertain whether the results of this study represent certain truth in regards to the implied versatility of the stories or only a coincidental fluke.

\section{Final Remarks}

I would like to conclude by sharing a few personal insights I gleaned during the time I spent engaged with the study. Working with narrative texts from a Native culture I was not raised in presented significant challenges, even though I have spent many years 
involved and participating in it. I have, in fact, made the Dakota way my chosen way of life and adhere as best I can to its teachings. As a cultural outsider though, many opportunities are simply not accessible to me and so, like Iktomi, I have had to improvise and use the available resources at my disposal in attempting to understand the Dakota stories from a non-Native perspective.

Iktomi himself facilitated the creation of this work and led me "going along," as it were, in search of deeper meaning of the stories and of my life. The four-year process I underwent to conduct this study coincides with the cyclical nature and duration of the Dakota ceremonies, some of which require years of preparation and strong commitment, and also with the four-day vision quest in comparable fashion. From the time I began work in earnest on this project, each passing year cycled through a new iteration of the journey, and each revolution represented a stop along Iktomi's wandering path. All the while, as I worked over the data, I also walked through ceremonial commitments I had made in addition to day-to-day obligations to my family, friends, and communities. Further, I was acutely aware that I had embarked on an adventure with the trickster and knew that anything could happen at any time to turn things upside down. And it didrepeatedly.

At the beginning, I set out on the journey toward an unknowable destination on an exploratory search for understanding — my "food"—hoping for academic insights as well as spiritual growth. I had a story and a purpose but no precedent to lead me toward a sure conclusion. Like Iktomi, I let my instincts guide me, confident the way would be shown. Finding my opportunity in the story characters, just as Iktomi found his in the Ducks, I 
pondered how to reap sustenance from them, and I proceeded through all of Iktomi's foibles as I delved into the work. I experienced my share of frustrations and irritations while striving to maintain control over the ballooning volume of information that started streaming in from many different directions. Always, the academic process lined up with real-life experiences occurring in my personal sphere of activities and through my ceremonial commitments, and each development presented a renewed opportunity to challenge the direction I was headed. "Dogged perseverance" became my mantra, while I maintained an inner visual of myself as Iktomi stumbling and falling down, rolling upside down, and then getting back on my feet and moving on, bruised and all. One must shrug it off and laugh or go insane. Yet, through it all, I think I am one up on the old Trickster because my perseverance and effort have yielded fruitful results.

As a consequence of my experience working with this tale, I firmly maintain that one cannot entertain the Native cultural myths without becoming transformed in some way, however slightly, and that to work with the stories in any way will invoke an invitation to experiential self-reflection. It cannot be avoided. Whatever their origin, I am convinced that cultural stories such as this one, Iktomi, are imbued with the very power to manifest whatever the listener or reader needs - at whatever point of development or level of understanding they have achieved at the time of the encounter. In this way, I suppose, the stories transcend culture and apply universally to humans everywhere, regardless of background, belief, or experience. Perhaps the spiritual and dynamic aspects of the stories account for why academics are stymied by them; because spirituality is a personal experience, it might not be possible to generalize and interpret 
the stories' meaning let alone dissect their inherent structure. However, as artifacts of culture, while further study with this one and different stories is needed, I offer my approach and method as a beginning tool and guide for others who may be willing to embark on that uncompromising journey toward self-knowledge. 


\section{References}

Arrivé, M., Coquet, J.-C., \& Leader, J. (1992). Linguistics and psychoanalysis: Freud, Saussure, Hjelmslev, Lacan and others, Semiotic Crossroads, Series 4. Amsterdam: John Benjamins Publishing Company.

Bakhtin, M. M., Holquist, M., \& Emerson, C. (1986). Speech genres and other late essays. University of Texas Press Slavic series, no. 8. Austin: University of Texas Press.

Ballenger, B. (1997). Methods of memory: On Native American storytelling. College English, 59(7), Urbana, IL: National Council of Teachers of English pp. 789-800.

Ballinger, F. (2004). Living sideways: Tricksters in American Indian oral traditions. Norman: University of Oklahoma Press.

Bierhorst, J. (1975). American Indian verbal art and the role of the literary critic. The Journal of American Folklore, 88(350), pp. 401-408. Retrieved from http://www.jstor.org/stable/538655

Bierwert, C., \& Indiana University, Bloomington. (1996). Lushootseed texts: An introduction to Puget Salish narrative aesthetics, Studies in the anthropology of North American Indians. Lincoln: University of Nebraska Press in cooperation with the American Indian Studies Research Institute, Indiana University, Bloomington.

Black, E., Neihardt, J. G., \& DeMallie, R. J. (2008). Black Elk speaks: Being the life story of a holy man of the Oglala Sioux. Albany, N.Y: Excelsior Editions, State University Press of New York Press.

Brice Heath, S. (1986). Taking a cross-cultural look at narratives. In K. G. Butler, (Ed.), Narrative discourse: Development and disorders, Topics in language disorders, 7(1), pp. 84-94. Salem, MA: Aspen Publishers, Inc.

Bright, W. (1987). The natural history of Old Man Coyote. In B. Swann, \& A. Krupat (Eds.), Recovering the Word: Essays on Native American oral literature. Berkeley, Los Angeles, London: University of California Press.

Brown, J. A., Suttles, W. P., Boas, F., Hymes, D. H., Peterson, M. D., Ray, V. F., . . M. Silverstein (2004). Chinookans of the Lower Columbia River: NR06. New Haven, $\mathrm{CN}$ : Human Relations Area Files.

Bunzel, R. L. (1932a). Zuñi origin myths. Washington: G.P.O.

Bunzel, R. L. (1932b). Introduction to Zuñi ceremonialism. Forty-seventh Annual Report ... 1929/30, 29, 467-544.

Bunzel, R. L. (1932c). Zuñi katcinas: An analytical study. Forty-seventh Annual Report ... 1929/30, 29, 837-1086.

Bunzel, R. L. (1974). Zuni texts. Publications of the American Ethnological Society: Vol. 15. New York: AMS Press.

Burke, K. (1957). The philosophy of literary form: Studies in symbolic action. New York: Vintage Books.

Clements, W. M. (1996). This voluminous unwritten book of ours: Early Native American writers and the oral tradition. In H. Jaskoski \& L. B. Ruoff (Eds.), 
Early Native American writing: New critical essays (pp. 122-135). New York: Cambridge University Press.

Deloria, E. C. (1932). Dakota texts. In F. Boas (Ed.), Publications of the American Ethnological Society, XIV. New York: G. E. Stechert \& Co.

Deloria, E. C., \& Godfrey, J. G. (2007). The Dakota way of life. Sioux Falls, SD: Mariah Press.

Deloria, V., Jr. (1998). Introduction. In E. C. Deloria, Speaking of Indians. Lincoln: University of Nebraska Press.

Deloria, V., Jr. (1999). Spirit \& reason: The Vine Deloria, Jr., reader. Golden, CO: Fulcrum Publishing.

DeMallie, R. J. (1975). Joseph N. Nicollet's account of the Sioux and Assiniboin in 1839. Pierre, SD: South Dakota State Historical Society.

DeMallie, R. J. (1988). Afterword. In E. C. Deloria, Waterlily (233-244).

DeMallie, R. J. (1993). "These have no ears": Narrative and ethnohistorical method. Ethnohistory 40(4), pp. 516-538.

DeMallie, R. J., \& Ortiz, A. (1994). North American Indian anthropology: Essays on society and culture. Norman: University of Oklahoma Press.

DeMallie, R. J., \& Parks, D. R. (1987). Sioux Indian religion: Tradition and innovation. Norman: University of Oklahoma Press.

DeMallie, R. J., Sturtevant, W. C., \& Smithsonian Institution. (2001). Plains. Washington, D.C: Smithsonian Institution.

Densmore, F. (1992). Teton Sioux music and culture. Lincoln: University of Nebraska Press.

Dominguez, S. R. (2003). Zitkala-S̆a: The representative Indian. In Zitkala S̆a, American Indian stories. Lincoln: University of Nebraska Press.

Eichhorst, S. (n.d.). Emuseum @ Minnesota State University, Mankato. Retrieved from http://www.mnsu.edu/emuseum/information/biography/abcde/deloria_ella_cara.ht $\mathrm{ml}$

Fisher, D. (1979). Zitkala S̆a: The evolution of a writer. American Indian Quarterly, 5(3), pp. 229-238.

Fisher, W. R. (1984). Narration as a human communication paradigm: The case of public moral argument. Communication Monographs, 51(1), 1-22.

Fisher, W. R. (1987). Human communication as narration: Toward a philosophy of reason, value, and action. In C. C. Arnold (Series Ed.), Studies in Rhetoric/Communication, 4. Columbia: University of South Carolina Press.

Georgakopoulou, A. (1997). Narrative performances: A study of modern Greek storytelling. In A. H. Jucker (Ed.), Pragmatics \& Beyond New Series, 46. Amsterdam: John Benjamins Publishing Company.

Gill, S. D. (1987). Mythic themes. In L. E. Sullivan (Ed.), Native American religions: North America (pp. 157-166). New York: Macmillan Publishing Company.

Horne, D. A. (1999). Contemporary American Indian writing: Unsettling literature. New York: Peter Lang Publishing, Inc. 
Huntsman, J. F. (1983). Traditional Native American literature: The translation dilemma. In B. Swann (Ed.), Smoothing the ground: Essays on Native American oral literature. Berkeley, Los Angeles, London: University of California Press.

Hymes, D. (1971). The contribution of folklore to sociolinguistic research. The Journal of American Folklore, 84(331), 42-50. Retrieved from http://www.jstor.org/stable/539732

Hymes, D. (1975). Folklore's nature and the sun's myth. The Journal of American Folklore, 88(350), 345-369. Retrieved from http://www.jstor.org/stable/538651

Hymes, D. (1977). Discovering Oral Performance and Measured Verse in American Indian Narrative. New Literary History, 8(3), 431-457.

Hymes, D. (1980). Particle, pause and pattern in American Indian narrative verse. American Indian Culture and Research Journal, 4(4), 7-51.

Hymes, D. (1982). Narrative Form as a "Grammar" of Experience: Native Americans and a Glimpse of English. Journal of Education, 164(2), 121-42.

Hymes, D. (1987). Anthologies and narrators. In B. Swann, \& A. Krupat (Eds.), Recovering the Word: Essays on Native American oral literature. Berkeley, Los Angeles, London: University of California Press.

Hymes, D. (1994). Foundations in sociolinguistics: An ethnographic approach. Philadelphia, Pa: Univ. of Pennsylvania Pr.

Hymes, D. H. (1955). The language of the Kathlamet Chinook (Doctoral Dissertation). OCLC Number: 34186813.

Hymes, D. H. (1980). A narrative view of the world. In D. Hymes, Language in Education: Ethnolinguistic Essays (pp. 129-138). Washington, D. C.; Center for Applied Linguistics.

Hymes, D. H. (1981). "In vain I tried to tell you": Essays in Native American ethnopoetics. Philadelphia: University of Pennsylvania Press.

Hymes, D. H. (1993). On Typology of Cognitive Styles in Language (with Examples from Chinookan). Anthropological Linguistics, 35(4), 440-475.

Hymes, D. H., \& Simpson, L. (1976). Louis Simpson's "The deserted boy.". (Reprinted from S.I.: Poetics: Vol. 5.).

Jahner, E. (1983). Stone boy: Persistent hero. In B. Swann (Ed.). Smoothing the ground: Essays on Native American oral literature. Berkeley, Los Angeles, London: University of California Press.

Kampfe, J. (Ed.) (1999). Reading and writing the Lakota language: Lakota iyapi un wowapi nahan yawapi. Salt Lake City: University of Utah Press.

Karolevitz, R. F. (1975). Challenge: The South Dakota story. Sioux Falls, SD: Brevet Press, Inc.

Kimball, G. (1993). Koasati narrator and narrative. In A. Krupat (Ed.), New voices in Native American literary criticism, (pp. 3-36). Washington: Smithsonian Institution Press.

Kroeber, K. (Ed.). (1991). Traditional literatures of the American Indian: Texts and interpretations. Lincoln: University of Nebraska Press.

Kroeber, K. (Ed.). (1998). Artistry in Native American myths. Lincoln: University of Nebraska Press. 
Krupat, A. (1987). Post-structuralism and oral literature. In B. Swann, \& A. Krupat (Eds.), Recovering the Word: Essays on Native American oral literature. Berkeley, Los Angeles, London: University of California Press.

Krupat, A. (1989). The dialogic of Silko's Storyteller. In G. Vizenor (Ed.), Narrative chance: Postmodern discourse on Native American Indian literatures. Albuquerque: University of New Mexico Press.

Krupat, A. (Ed.). (1993). New voices in Native American literary criticism. Washington: Smithsonian Institution Press.

Labov, W. (1972). Language in the inner city. Philadelphia: Univ. of Pennsylvania Press.

Lalande, A. (1926). Vocabulaire technique et critique de la philosophie. Paris: F. Alean.

Lewis, H. S. (2001). The passion of Franz Boas. American Anthropologist, New Series 103(2): 447-467. American Anthropological Association. Malden, MA: Blackwell Publishing. Retrieved from Article Stable URL: http://www.jstor.org/stable/683476

Lincoln, K. (1983). Native American literatures. In B. Swann (Ed.). Smoothing the ground: Essays on Native American oral literature. Berkeley, Los Angeles, London: University of California Press.

Markowitz, H. (1996). Oglala Lakota scholar and leader. In F. E. Hoxie (Ed.), Encyclopedia of North American Indians (pp. 617-618). New York, N.Y.: Houghton Mifflin Company.

Marshall, J. M. (2001). The Lakota way: Stories and lessons for living. New York: Penguin Group.

Mattina, A. (1987.) North American Indian mythography: Editing texts for the printed page. In B. Swann, \& A. Krupat (Eds.), Recovering the Word: Essays on Native American oral literature. Berkeley, Los Angeles, London: University of California Press.

Mead, M. (1932/1969). The changing culture of an Indian tribe. New York: AMS Press, Inc.

Mintz, S. W. (Ed.). (1985). History, evolution, and the concept of culture: Selected papers by Alexander Lesser. Cambridge University Press.

Mooney, J., \& DeMallie, R. J. (1991). The ghost-dance religion and the Sioux outbreak of 1890. Lincoln, NE [etc.: University of Nebraska press.

Muchnik, M., \& Stavans, A. (2009). Telling the same story to your child: Mothers' versus fathers' storytelling interactions. Women and Language, 32(1), 60-69.

Mumby, D. K. (1993). Narrative and social control: Critical perspectives. In Sage annual reviews of communication research, 21. Newbury Park, CA: Sage Publications.

Native American Citizenship. (n.d.). Retrieved from http://www.nebraskastudies.org/0700/frameset_reset.html?http://www.nebraskast udies.org/0700/stories/0701_0146.html

Native American People/Tribes: Great Sioux Nation. (2005). Retrieved from http://www.snowwowl.com/peoplesioux.html 
Neihardt, J. G., Black, E., \& DeMallie, R. J. (1985). The Sixth Grandfather: Black Elk's teachings given to John G. Neihardt. Lincoln: University of Nebraska Press.

Norrick, N. R. (2000). Conversational narrative: Storytelling in everyday talk. In E. F. K. Koerner (Ed.), Amsterdam studies in the theory and history of linguistic science, Series IV, Current Issues in Linguistic Theory, 203. Amsterdam: J. Benjamins.

Olson, J. C. (1965). Red Cloud and the Sioux problem. Lincoln: University of Nebraska Press.

Picotte, A. M. (1985). Foreword. In Zitkala-S̆a, Old Indian Legends (xi-xviii). Lincoln: University of Nebraska Press. (Original work published 1901).

Picotte, A. M. (1988). Biographical sketch of the author. In E. C. Deloria, Waterlily (229231).

Picotte, A. \& Pavich, P. (1992). Introductory Notes. In E. C. Deloria, Dakota Texts (vviii). Freeman, SD: University of South Dakota Press.

Poole, D. W. C., \& DeMallie, R. J. (1988). Among the Sioux of Dakota: 18 month's experience as an Indian agent; 1869-70. St. Paul: Minnesota History. Soc. Pr.

Radin, P. (1953). The world of primitive man. New York: H. Schuman.

Radin, P. (1957). Primitive man as philosopher. New York: Dover Publications.

Radin, P. (1972). The trickster: A study in American Indian mythology. New York: Schocken Books.

Radin, P., Kerényi, K., \& Jung, C. G. (1956). The trickster: A study in American Indian mythology. New York: Bell Pub. Co.

Reichard, G. A. (1938). Social Life. In F. Boas, (Ed.), 1938; General Anthropology. New York: D. C. Heath and Company.

Riggs, S. R., \& Dorsey, J. O. (2004). Dakota grammar: With texts and ethnography. St. Paul, MN: Minnesota Historical Society Press. (Original work published 1893)

Robinson, E. B. (1966). History of North Dakota. Lincoln: University of Nebraska Press.

Roemer, K. M. (1983). Context and continuity. In B. Swann (Ed.), Smoothing the ground: Essays on Native American oral literature. Berkeley, Los Angeles, London: University of California Press.

Schöler, B. (1981). Images and counter-images: Ohiyesa, Standing Bear and and [sic] American literature. American Indian Culture and Research Journal, 5(2), pp. 3762.

Sherzer, J., \& Woodbury, A. C. (1987). Native American discourse: Poetics and rhetoric. Cambridge University Press.

Silver, S., \& Miller, W. R. (1997). American Indian languages: Cultural and social contexts. Tucson: University of Arizona Press.

Sioux History Timeline (2007). Sioux-Indians.com. Retrieved from http://www.siouxindians.com/sioux-history.htm

Starobinski, J. (1979). Words upon words. New Haven and London: Yale University Press.

Sutton-Smith, B. (1986). The development of fictional narrative performances. In K. G. Butler (Ed.). (1986). Narrative discourse: Development and disorders. Topics in language disorders, 7(1), 1-10. Salem, MA: Aspen Publishers, Inc. 
Swann, B. (Ed.). (1983). Smoothing the ground: Essays on Native American oral literature. Berkeley, Los Angeles, London: University of California Press.

Tedlock, D. (1968). The ethnography of tale-telling at Zuñi. New Orleans: Tedlock.

Tedlock, D. (1971). On the translation of style in oral narrative. The Journal of American Folklore, 84(331), pp. 114-133.

Tedlock, D. (1977). Toward an oral poetics. New Literary History, 8(3), pp. 507-519.

Tedlock, D. (1983). The spoken word and the work of interpretation. Philadelphia: University of Pennsylvania Press.

Tedlock, D. (1999). Finding the center: The art of the Zuni storyteller. Lincoln, Neb: University of Nebraska Press.

Tedlock, D., Peynetsa, A., \& Sanchez, W. (1972). Finding the center: Narrative poetry of the Zuñi Indians. New York: Dial Press.

Trechter, S. (1996). The pragmatic functions of gender deixis in Lakhota. Ann Arbor: ProQuest Company. (Doctoral Dissertation, University of Kansas, 1996) Dissertation Abstracts International,56(11), 4384.

Turner, V. (1980). Social dramas and stories about them. Critical Inquiry, 7, 141-148.

Ullrich, J. F., \& Lakota Language Consortium. (2011). New Lakota dictionary: Lakhótiyapi-English/English-Lakhótiyapi \& incorporating the Dakota dialects of Yankton-Yanktonai \& Santee-Sisseton $\left(2^{\text {nd }}\right.$ ed.). Bloomington, IN: Author.

Utley, R. M. (1984). Grant's Peace Policy: 1869-1876. The Indian frontier of the American West, 1846-1890 (pp. 127-133). Retrieved from http://bit.ly/PBOvHL

Velie, A. (1989). The trickster novel. In G. Vizenor (Ed.), Narrative chance: Postmodern discourse on Native American Indian literatures (pp. 121-139). Albuquerque: University of New Mexico Press.

Vizenor, G. (1989). Trickster discourse: Comic holotropes and language games. In G. Vizenor. (1989). Narrative chance: Postmodern discourse on Native American Indian literatures (pp. 187-211). Albuquerque: University of New Mexico Press.

Vizenor, G. (1990). Trickster discourse. American Indian Quarterly, 14(3), 277-287. Retrieved from http://www.jstor.org/stable/1185655

Walker, J. R., \& DeMallie, R. J. (1982). Lakota society. Lincoln: University of Nebraska Press.

Walker, J. R., Jahner, E., \& State Historical Society of Colorado. (1983). Lakota myth. Lincoln: University of Nebraska Press.

Walker, J. R., DeMallie, R. J., Jahner, E., \& Colorado Historical Society. (1991). Lakota belief and ritual. Lincoln: University of Nebraska Press.

Wiget, A. (1987). A performance analysis of a Hopi coyote story. In B. Swann, \& A. Krupat (Eds.). (1987). Recovering the word: Essays on Native American oral literature. Berkeley: University of California Press.

Young Bear, S. \& Theisz, R. D. (1994). Standing in the light: A Lakota way of seeing. Lincoln: University of Nebraska Press.

Zimiga, J. (Director). (2010, May 5). Jerome Kills Small Profile. [Audio podcast]. Retrieved from http://www.youtube.com/user/Pit44Productions

Zinn, H. (1980). A people's history of the United States. New York: Harper \& Row.

Zitkala S̆a. (1901/1985). Old Indian legends. Lincoln: University of Nebraska Press. 
Zitkala S̆a. (1921/2003). American Indian stories. Lincoln: University of Nebraska Press. 
Appendix A

Iktomi Story Plot Summary:

Correspondences between Episodic Sequence of Events and Phases in Narrative Models

\begin{tabular}{|c|c|c|c|c|c|}
\hline Cycle & Phases in Narrative Models & Episode 1 & Episode 2 & Episode 3 & Episode 4 \\
\hline & $\begin{array}{l}\text { Labov's Narrative Model } \\
\text { Bierhorst's Itinerary of Signs } \\
\text { Alternative Description }\end{array}$ & $\begin{array}{l}\text { Iktomi } \\
\text { encounters } \\
\text { the Ducks }\end{array}$ & $\begin{array}{c}\text { Iktomi gets } \\
\text { trapped } \\
\text { in the Tree(s) }\end{array}$ & $\begin{array}{c}\text { Iktomi } \\
\text { encounters } \\
\text { the Wolf(ves) }\end{array}$ & $\begin{array}{c}\text { Iktomi } \\
\text { encounters } \\
\text { the Wolf a } 2^{\text {nd }} \\
\text { time }\end{array}$ \\
\hline 1 & $\begin{array}{l}\text { L: Orientation } \\
\text { B: Opening incident (youth) } \\
\text { A: Background/orientation }\end{array}$ & $\begin{array}{l}\text { The trickster- } \\
\text { spider Iktomi } \\
\text { travels along } \\
\text { aimlessly. }\end{array}$ & $\begin{array}{l}\text { Presently, Iktomi } \\
\text { becomes aware of } \\
\text { a persistent sound } \\
\text { nearby, which } \\
\text { agitates him. }\end{array}$ & $\begin{array}{l}\text { Sitting with his } \\
\text { fear and anxiety, } \\
\text { Iktomi watches } \\
\text { the Wolf/ves } \\
\text { approach his fire. } \\
\text { He feels } \\
\text { powerless to do } \\
\text { anything about the } \\
\text { situation. }\end{array}$ & $\begin{array}{l}\text { Still hungry, } \\
\text { Iktomi continues } \\
\text { to wander } \\
\text { aimlessly along. }\end{array}$ \\
\hline
\end{tabular}




\begin{tabular}{|c|c|c|c|c|c|}
\hline Cycle & Phases in Narrative Models & Episode 1 & Episode 2 & Episode 3 & Episode 4 \\
\hline & $\begin{array}{l}\text { Labov's Narrative Model } \\
\text { Bierhorst's Itinerary of Signs } \\
\text { Alternative Description }\end{array}$ & $\begin{array}{c}\text { Iktomi } \\
\text { encounters } \\
\text { the Ducks }\end{array}$ & $\begin{array}{c}\text { Iktomi gets } \\
\text { trapped } \\
\text { in the Tree(s) }\end{array}$ & $\begin{array}{c}\text { Iktomi } \\
\text { encounters } \\
\text { the Wolf(ves) }\end{array}$ & $\begin{array}{c}\text { Iktomi } \\
\text { encounters } \\
\text { the Wolf a } 2^{\text {nd }} \\
\text { time }\end{array}$ \\
\hline 2 & $\begin{array}{l}\text { L: Complicating Action } \\
\text { B: Ritual combat (maturity) } \\
\text { A: Situation/incident }\end{array}$ & $\begin{array}{l}\text { Soon, he happens } \\
\text { upon a group of } \\
\text { Ducks; } \\
\text { whereupon, he } \\
\text { devises a plot to } \\
\text { kill some to eat. }\end{array}$ & $\begin{array}{l}\text { He sets out to } \\
\text { subdue it, but he } \\
\text { fails his attempt to } \\
\text { exert his control } \\
\text { and influence over } \\
\text { the noise, and he } \\
\text { becomes caught in } \\
\text { a tree. }\end{array}$ & $\begin{array}{l}\text { Iktomi watches } \\
\text { while the } \\
\text { Wolf/ves eat all of } \\
\text { the roasting meat. }\end{array}$ & $\begin{array}{l}\text { Eventually, he } \\
\text { finds the Wolf } \\
\text { that had eaten his } \\
\text { supper. This time, } \\
\text { Iktomi has the } \\
\text { upper hand and } \\
\text { seeks revenge on } \\
\text { the Wolf. }\end{array}$ \\
\hline 3 & $\begin{array}{l}\text { L: Resolution } \\
\text { B: Flight and death (demise) } \\
\text { A: Encounter or crisis }\end{array}$ & $\begin{array}{l}\text { Iktomi deceives } \\
\text { the Ducks through } \\
\text { trickery, playing } \\
\text { on their social } \\
\text { values of trust and } \\
\text { hospitality. }\end{array}$ & $\begin{array}{l}\text { From the height, } \\
\text { he espies a } \\
\text { potential } \\
\text { adversary in the } \\
\text { form of } \\
\text { Wolf/(ves), which } \\
\text { are passing } \\
\text { through a long } \\
\text { way off in the } \\
\text { distance. }\end{array}$ & $\begin{array}{l}\text { The Wolf/ves } \\
\text { satisfy themselves } \\
\text { that they have } \\
\text { eaten all the roasts } \\
\text { and depart. }\end{array}$ & There is a scuffle, \\
\hline
\end{tabular}




\begin{tabular}{|c|c|c|c|c|c|}
\hline Cycle & Phases in Narrative Models & Episode 1 & Episode 2 & Episode 3 & Episode 4 \\
\hline & $\begin{array}{l}\text { Labov's Narrative Model } \\
\text { Bierhorst's Itinerary of Signs } \\
\text { Alternative Description }\end{array}$ & $\begin{array}{l}\text { Iktomi } \\
\text { encounters } \\
\text { the Ducks }\end{array}$ & $\begin{array}{c}\text { Iktomi gets } \\
\text { trapped } \\
\text { in the Tree(s) }\end{array}$ & $\begin{array}{c}\text { Iktomi } \\
\text { encounters } \\
\text { the Wolf(ves) }\end{array}$ & $\begin{array}{c}\text { Iktomi } \\
\text { encounters } \\
\text { the Wolf a } 2^{\text {nd }} \\
\text { time }\end{array}$ \\
\hline 4 & $\begin{array}{l}\text { L: Evaluation } \\
\text { B: Underworld Journey } \\
\quad \text { (limbo) } \\
\text { A: Result and consequence }\end{array}$ & $\begin{array}{l}\text { In the process, he } \\
\text { manages to kill a } \\
\text { few of the Ducks } \\
\text { and prepares them } \\
\text { for roasting. }\end{array}$ & $\begin{array}{l}\text { Anxious that the } \\
\text { Wolf/(ves) will } \\
\text { find his cache of } \\
\text { roasts, Iktomi } \\
\text { foolishly } \\
\text { precipitates the } \\
\text { Wolf(ves)' } \\
\text { discovery of it. }\end{array}$ & $\begin{array}{l}\text { Iktomi is then } \\
\text { freed from the } \\
\text { Tree(s). }\end{array}$ & $\begin{array}{l}\text { but the Wolf } \\
\text { manages to escape } \\
\text { Iktomi's } \\
\text { vengeance and } \\
\text { runs away. }\end{array}$ \\
\hline 5 & $\begin{array}{l}\text { L: Coda } \\
\text { B: Resurrection } \\
\text { A: Return to starting point }\end{array}$ & $\begin{array}{l}\text { Then, he sits back } \\
\text { to wait while they } \\
\text { cook. }\end{array}$ & $\begin{array}{l}\text { Iktomi thus } \\
\text { inadvertently } \\
\text { draws to himself } \\
\text { the very thing he } \\
\text { fears. }\end{array}$ & $\begin{array}{l}\text { Disgusted and } \\
\text { disappointed, he } \\
\text { goes on his way. }\end{array}$ & $\begin{array}{l}\text { Iktomi once more } \\
\text { has failed to sate } \\
\text { his desire, and so } \\
\text { he wanders } \\
\text { aimlessly again to } \\
\text { his next } \\
\text { adventure. }\end{array}$ \\
\hline
\end{tabular}


Appendix B

Synoptic Alignment of Three Versions of Lakota Folk Myth Iktomi

\begin{tabular}{|c|c|c|c|}
\hline $\begin{array}{l}\text { Line } \\
\text { No. }\end{array}$ & $\begin{array}{c}\text { Iktomi and the Ducks } \\
\text { ZITKALA-SA }\end{array}$ & $\begin{array}{l}\text { Ikto'mi Tricks the Pheasants } \\
\text { DELORIA }\end{array}$ & $\begin{array}{c}\text { Iktomi } \\
\text { W ALKER } \\
\end{array}$ \\
\hline 1 & $\begin{array}{l}\text { a. One day he }\{\text { IKRp } 3\} \text { sat hungry } \\
\text { within his }\{\text { IKRp } 3\} \text { teepee. } \\
\{\text { IKTOP }\}\end{array}$ & a. & a. \\
\hline 1 & $\begin{array}{l}\text { b. Suddenly he }\{\text { IKRp3 }\} \text { rushed } \\
\text { out }\{\text { IKAt } 11\} \text {, dragging after } \\
\text { him }\{\text { IKRp } 3\} \text { his }\{\text { IKRp } 3\} \\
\text { blanket. }\end{array}$ & b. & b. \\
\hline 1 & $\begin{array}{l}\text { c. }[\ldots 3 \text { b]Quickly }\{\text { IKAt } 11\} \\
\text { spreading it on the ground } \\
\{\text { IKTOP }\}, \text { he }\{\text { IKRp3 }\} \text { tore up } \\
\text { dry tall grass }\{\text { GRRp } 1\} \text { with } \\
\text { both his }\{\text { IKRp3\} hands } \\
\text { (IKRp } 2\} \text { and tossed it fast } \\
\text { \{IKAt11\} into the blanket. } \\
\text { Tying all the four corners } \\
\text { together in a knot }\{\text { IKAb7\}, }\end{array}$ & c. $[\ldots 3 b]$ & c. $[\ldots 3 b]$ \\
\hline 1 & $\begin{array}{l}\text { d. }[\ldots 3 \mathrm{e}] \text { he }\{\text { IKRp } 3\} \text { threw the } \\
\text { light bundle of grass }\{\text { GRRp1\} } \\
\text { over his }\{\text { IKRp } 3\} \text { shoulder. }\end{array}$ & d. $[\ldots 3 \mathrm{e}]$ & d. $[\ldots 3 \mathrm{e}]$ \\
\hline 1 & $\begin{array}{c}\text { e. }[\ldots 3 \mathrm{f}] \text { Snatching up a slender } \\
\text { willow stick with his }\{\text { IKRp } 3\} \\
\text { free left hand }\{\text { IKRp } 2\}\end{array}$ & e. $[\ldots 3 \mathrm{f}]$ & e. $[\ldots 3 \mathrm{f}]$ \\
\hline 1 & $\begin{array}{l}\text { f. he }\{\text { IKRp3 }\} \text { started off } \\
\quad\{\text { IKAb9\} with a hop and a leap. }\end{array}$ & $\begin{array}{ll}\text { f. } & \text { IKTOMI }\{\text { IKRP1 }\} \text { was walking } \\
& \{\text { IKAb9 }\} \text { at random } \\
\end{array}$ & $\begin{array}{ll}\text { f. } & \text { IKTOMI }\{\text { IKRp 1 }\} \text { was just } \\
& \text { going along }\{\text { IKAb9 }\},\end{array}$ \\
\hline
\end{tabular}




\begin{tabular}{|c|c|c|c|}
\hline $\begin{array}{l}\text { Line } \\
\text { No. }\end{array}$ & $\begin{array}{c}\text { Iktomi and the Ducks } \\
\text { ZITKALA-SA }\end{array}$ & $\begin{array}{c}\text { Ikto'mi Tricks the Pheasants } \\
\text { DELORIA }\end{array}$ & $\begin{array}{c}\text { Iktomi } \\
\text { WALKER }\end{array}$ \\
\hline & $\{$ IKAb9\} & & \\
\hline 1 & $\begin{array}{l}\text { g. From side to side bounced [see } \\
3 \mathrm{~g} \text { ] the bundle on his }\{\text { IKRp } 3\} \\
\text { back, as he }\{\text { IKRp } 3\} \text { ran } \\
\text { (IKAb9) light-footed over the } \\
\text { uneven ground }\{\text { IKTOP }\} \text {. }\end{array}$ & $\begin{array}{ll}\text { g. } & \text { along a creek }\{\text { WARp } 1\} \\
& \{\text { IKTOP }\}\end{array}$ & g. $\quad$ over there. $\{$ IKTOP $\}$ \\
\hline 1 & h. ------- & h. ------- & h. (So they tell) \\
\hline 1 & i. Soon & i. $\quad$ and & i. Then \\
\hline 1 & $\begin{array}{l}\text { j. he }\{\text { IKRp } 3\} \text { came to the edge } \\
\text { of the great level land } \\
\text { \{IKTOP }\} \text {. }\end{array}$ & $\begin{array}{ll}\text { j. } & \text { he }\{\text { IKRp3 }\} \text { heard }\{\text { IKAb8 }\} \\
\text { dancing, }\end{array}$ & j. \\
\hline 1 & k. On the hilltop & $\mathrm{k}$. & k. There was a hill; $\{$ IKTOP $\}$ \\
\hline 1 & $\begin{array}{l}\text { 1. he }\{\text { IKRp } 3\} \text { paused for breath. } \\
\text { \{WIRp1\} }\end{array}$ & $\begin{array}{ll}\text { 1. } & \text { so he }\{\text { IKRp3 }\} \text { stopped } \\
& \{\text { IKAb9\} to listen }\{\text { IKAb8 }\} .\end{array}$ & 1. he $\{$ IKRp3 $\}$ climbed it. \\
\hline 1 & m.------- & m. [It is said] & m. ------- \\
\hline 2 & a. ------- & a. ------- & a. Then \\
\hline 2 & $\begin{array}{l}\text { b. With wicked }\{\text { IKAt } 12\} \text { smacks } \\
\text { of his }\{\text { IKRp } 3\} \text { dry parched } \\
\text { lips, as if tasting some tender } \\
\text { meat }\{\text { IKAt } 12\} \text {, }\end{array}$ & b. & b. \\
\hline 2 & $\begin{array}{l}\text { c. he }\{\text { IKRp } 3\} \text { looked }\{\text { IKAb8 }\} \\
\text { straight into space }\{\text { IKTOP } \\
\text { toward the marshy river } \\
\text { bottom }\{\text { IKTOP }\{\text { WARp } 1\} \\
\text { With a thin palm shading his } \\
\{\text { IKRp3 } \text { eyes from the }\end{array}$ & c. & c. \\
\hline
\end{tabular}




\begin{tabular}{|c|c|c|c|}
\hline $\begin{array}{l}\text { Line } \\
\text { No. }\end{array}$ & $\begin{array}{c}\text { Iktomi and the Ducks } \\
\text { ZITKALA-SA }\end{array}$ & $\begin{array}{c}\text { Ikto'mi Tricks the Pheasants } \\
\text { DELORIA }\end{array}$ & $\begin{array}{c}\text { Iktomi } \\
\text { WALKER }\end{array}$ \\
\hline & $\begin{array}{l}\text { western sun }\{\text { IKTOP }\}, \text { he } \\
\{\text { IKRp } 3\} \text { peered }\{\text { IKAb8 }\} \text { far } \\
\text { away into the lowlands } \\
\text { \{IKTOP }\}, \text { munching his } \\
\text { \{IKRp } 3 \text { own cheeks } \\
\text { IIKAt } 12\} \text { all the while. }\end{array}$ & & \\
\hline 2 & d. & $\begin{array}{ll}\text { d. } & \text { He }\{\text { IKRp } 3\} \text { finally located the } \\
& \text { source of the sound }\{\text { IKAb } 8\}\end{array}$ & d. \\
\hline 2 & 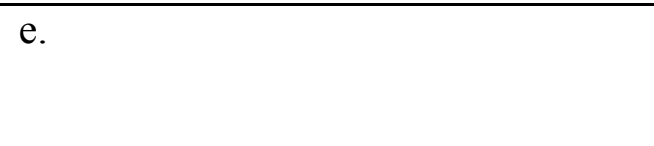 & $\begin{array}{l}\text { e. so he }\{\text { IKRp } 3\} \text { went towards it } \\
\text { and stood within sight of the } \\
\text { place; }\{\text { IKTOP }\}\end{array}$ & e. \\
\hline 2 & $\begin{array}{l}\text { f. "Ah-ha!" grunted he }\{\text { IKRp3 }\} \\
\text { satisfied }\{\text { IKAb8\} with what } \\
\text { he }\{\text { IKRp } 3\}[\ldots 2 L] \text { saw } \\
\text { \{IKAb8 }\}\end{array}$ & f. $[\ldots 2 \mathrm{~L}]$ and saw $\{$ IKAb8 $\}$ & f. $[\ldots 2 \mathrm{~L}]$ \\
\hline 2 & $\begin{array}{l}\text { g. A group of wild \{DUAt11\} } \\
\text { ducks }\{\text { DURp1\} }\end{array}$ & $\begin{array}{l}\text { g. that those }\{\text { DURp1\} were all } \\
\text { pheasants }\{\text { DURp1\} }\end{array}$ & $\begin{array}{l}\text { g. There were a lot of ducks } \\
\{\text { DURp1 }\end{array}$ \\
\hline 2 & h. were dancing $\{$ DUAb9 $\}$ & $\begin{array}{l}\text { h. } \begin{array}{l}\text { who }\{\text { DURp1 }\} \text { were dancing } \\
\text { \{DUAb9\} }\end{array}\end{array}$ & $\mathrm{h}$. \\
\hline 2 & i. and feasting $\{$ DUAb8 $\}$ & $\begin{array}{l}\text { i. and having a jolly } \\
\text { time }\{\text { DUAb8\}. }\end{array}$ & i. \\
\hline 2 & j. in the marshes $\{$ DUTOP $\}$. & $\mathrm{j}$. & $\begin{array}{l}\text { j. along the edge of the water. } \\
\{\text { DUTOP }\}\end{array}$ \\
\hline 2 & $\begin{array}{l}\text { k. With wings \{DURp1\} out- } \\
\text { spread, tip to tip, they } \\
\{\text { DURp4 }\} \text { moved }\{\text { DUAt } 11\} \text { up } \\
\text { and down in a large circle. }\end{array}$ & $\mathrm{k}$. & $\mathrm{k}$ \\
\hline
\end{tabular}




\begin{tabular}{|c|c|c|c|}
\hline $\begin{array}{l}\text { Line } \\
\text { No. }\end{array}$ & $\begin{array}{l}\text { Iktomi and the Ducks } \\
\text { ZITKALA-SA }\end{array}$ & $\begin{array}{c}\text { Ikto'mi Tricks the Pheasants } \\
\text { DELORIA }\end{array}$ & $\begin{array}{c}\text { Iktomi } \\
\text { W ALKER }\end{array}$ \\
\hline & $\begin{array}{l}\text { Within the ring, around a } \\
\text { small drum, sat the chosen } \\
\text { singers }\{\text { DURp } 2\} \text {, nodding } \\
\text { their }\{\text { DURp } 4\} \text { heads and } \\
\text { blinking their }\{\text { DURp4 }\} \text { eyes } \\
\{\text { DUAb8 } \text {. They }\{\text { DURp } 4\} \\
\text { sang }\{\text { DUAb7\} in unison } \\
\{\text { DUAt } 11\} \text { a merry dance-song, } \\
\text { and beat a lively tattoo on the } \\
\text { drum }\{\text { DUAb7\} }\end{array}$ & & \\
\hline 2 & 1. $[\ldots 2 \mathrm{f}]$ & 1. [...2f] & $\begin{array}{l}\text { 1. [...2f] Standing there, }\{\text { IKTOP } \\
\text { he }\{\text { IKRp } 3\} \text { saw }\{\text { IKAb8 } \\
\text { them }\{\text { DURp } 4\} \text { and he } \\
\{\text { IKRp } 3\} \text { finished thinking } \\
\{\text { IKAb8 }\} \text { about how to deceive } \\
\text { IIKAt12\} them }\{\text { DURp } 4\} .\end{array}$ \\
\hline 2 & m.------- & m. [It is said] & m.------- \\
\hline 3 & a. & $\begin{array}{l}\text { a. Immediately he }\{\text { IKRp } 3\} \\
\text { withdrew into the wood } \\
\{\text { IKTOP }\}\end{array}$ & a. \\
\hline 3 & b. $[\ldots 1 \mathrm{c}]$ & $\begin{array}{l}\text { b. }[\ldots 1 \mathrm{c}] \text { and wrapped some red } \\
\quad\{\text { IKCOL }\} \text { grass about a piece of } \\
\quad \text { wood }\{\text { IKAb } 7\}\end{array}$ & $\begin{array}{ll}\text { b. } & {[\ldots 1 \mathrm{c}] \mathrm{He}\{\mathrm{IKRp} 3\} \text { cut some }} \\
& \text { sticks }\{\text { IKAb7 }\} \text { and made a } \\
& \text { bundle }\{\mathrm{IKAb} 7\}\end{array}$ \\
\hline 3 & $\begin{array}{l}\text { c. Following a winding footpath } \\
\text { nearby, }\{\text { IKTOP }\}\end{array}$ & c. & c. \\
\hline 3 & d. [...3h] came a bent figure of & d. $[\ldots 3 \mathrm{~h}]$ & d. $[\ldots 3 \mathrm{~h}]$ \\
\hline
\end{tabular}




\begin{tabular}{|c|c|c|c|}
\hline $\begin{array}{l}\text { Line } \\
\text { No. }\end{array}$ & $\begin{array}{c}\text { Iktomi and the Ducks } \\
\text { ZITKALA-SA }\end{array}$ & $\begin{array}{c}\text { Ikto'mi Tricks the Pheasants } \\
\text { DELORIA }\end{array}$ & $\begin{array}{c}\text { Iktomi } \\
\text { WALKER }\end{array}$ \\
\hline & a Dakota brave $\{$ IKRp2 $\}$. & & \\
\hline 3 & $\begin{array}{l}\text { e. }[\ldots 1 \mathrm{~d}] \mathrm{He}\{\text { IKRp } 3\} \text { bore } \\
\{\text { IKAt } 12\} \text { on his }\{\text { IKRp } 3\} \\
\text { back a very large bundle. }\end{array}$ & $\begin{array}{l}\text { e. }[\ldots 1 \mathrm{~d}] \text { this he }\{\text { IKRp } 3\} \text { put on } \\
\text { his }\{\text { IKRp3\} back, }\end{array}$ & $\begin{array}{r}\text { e. } \quad[\ldots 1 \mathrm{~d}] \text { and carried it on his } \\
\{\text { IKRp } 3\} \text { back }\{\text { IKRp } 2\}\end{array}$ \\
\hline 3 & f. $[\ldots 1 \mathrm{e}]$ & f. $[\ldots 1 \mathrm{e}]$ & $\begin{array}{ll}\text { f. } & {[\ldots 1 \mathrm{e}] \text { and he }\{\text { IKRp } 3\} \text { cut }} \\
& \text { some wood }\{\text { IKAb7 }\}\{\text { IKAb7 }\} \\
& \text { that he }\{\text { IKRp } 3\} \text { had }\end{array}$ \\
\hline 3 & $\begin{array}{l}\text { g. With a willow cane }\{\text { IKAb7 }\} \\
\text { he }\{\text { IKRp } 3\} \text { propped himself } \\
\text { up }\end{array}$ & g. & g. \\
\hline 3 & h. $[\ldots 3 \mathrm{~d}]$ & h. $[\ldots 3 \mathrm{~d}]$ & $\begin{array}{l}\text { h. }[\ldots 3 \mathrm{~d} .] \text { and he }\{\text { IKRp } 3\} \text { came } \\
\text { out }\{\text { IKAb9 }\} \text { of the woods } \\
\{\text { IKTOP }\}\end{array}$ \\
\hline 3 & 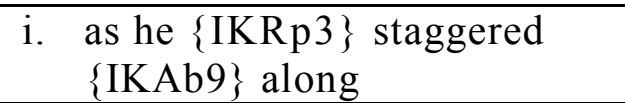 & i. and then walked $\{$ IKAb9\} past & $\begin{array}{l}\text { i. and ran }\{\text { IKAb9 }\} \text { past the ducks } \\
\{\text { DURp } 1\} .\end{array}$ \\
\hline 3 & $\mathrm{j}$. & $\mathrm{j}$. & j. $\quad$ (So they tell) \\
\hline 3 & $\begin{array}{l}\text { k. beneath his }\{\text { IKRp3 }\} \text { burden. } \\
\text { \{IKAt12\} }\end{array}$ & $\begin{array}{l}\text { k. without paying the slightest } \\
\text { attention to the dancers } \\
\text { \{DURp1\} }\{\text { DUAb9\}. }\{\text { IKAt12\} }\end{array}$ & $\begin{array}{ll}\text { k. } & \text { He }\{\text { IKRp3 }\} \text { wasn't sweating } \\
& \text { but he }\{\text { IKRp3 }\} \text { kept wiping } \\
& \text { sweat off }\{\text { IKAb8 }\} \text { as he } \\
& \{\text { IKRp } 3\} \operatorname{ran}\{\text { IKAt12\}. }\end{array}$ \\
\hline 3 & 1. ------- & 1. [It is said] & 1. (So they tell) \\
\hline 4 & a. ------- & a. ------- & a. Then \\
\hline 4 & $\mathrm{~b}$. & $\begin{array}{l}\text { b. } \\
\text { "Hey, there goes IKTO } \\
\{\text { IKRp5\}. Let's }\{\text { DURp4\} call } \\
\text { him }\{\text { IKRp3 } \text { over," they } \\
\{\text { DURp4\} said }\{\text { DUAb8 }\end{array}$ & $\mathrm{b}$. \\
\hline
\end{tabular}




\begin{tabular}{|c|c|c|c|}
\hline $\begin{array}{l}\text { Line } \\
\text { No. }\end{array}$ & $\begin{array}{c}\text { Iktomi and the Ducks } \\
\text { ZITKALA-SA }\end{array}$ & $\begin{array}{c}\text { Ikto'mi Tricks the Pheasants } \\
\text { DELORIA }\end{array}$ & $\begin{array}{c}\text { Iktomi } \\
\text { W ALKER }\end{array}$ \\
\hline & & $\{$ DUAt 11$\}$. & \\
\hline 4 & $\begin{array}{l}\text { c. "Ho! who \{OTRp2\} is } \\
\text { there?" \{DUAt } 12\} \\
\text { called out }\{\text { DUAb8\} a } \\
\text { curious }\{\text { DUAt } 12\} \text { old } \\
\text { duck }\{\text { SKRp1\}, still bobbing } \\
\text { up and down in the circular } \\
\text { dance. }\end{array}$ & c. & c. \\
\hline 4 & 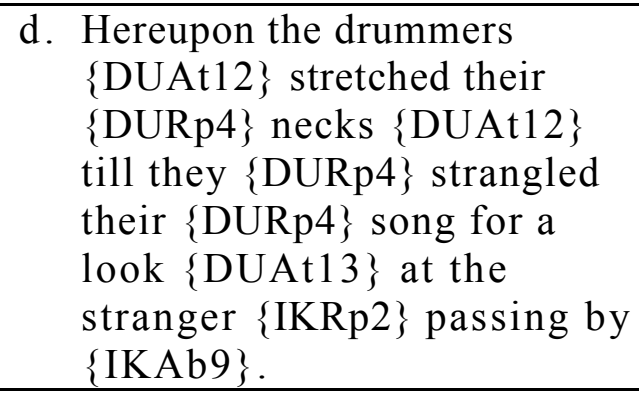 & d. & $\begin{array}{l}\text { d. A11 the ducks \{DURp1\} } \\
\text { \{DUAt11\} walked \{DUAb9) } \\
\text { up to him }\{\text { IKRp3 }\}\end{array}$ \\
\hline 4 & $\begin{array}{ll}\text { e. } & \text { "Ho, IKTOMI }\{\text { IKRp5 }\} ! \\
& \{\text { DUAb8 }\end{array}$ & $\begin{array}{ll}\text { e. } & \text { "IKTO!" }\{\text { IKRp5 }\}\{\text { DUAb8 }\} \\
\text { they }\{\text { DURp4 }\} \text { called } \\
\\
\{\text { DUAt } 11\}\end{array}$ & e. \\
\hline$\overline{4}$ & $\mathrm{f}$. & 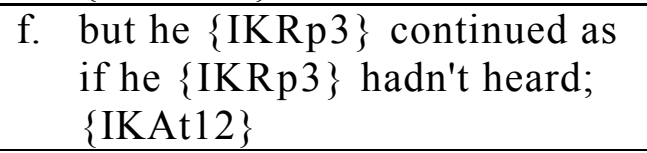 & f. \\
\hline & g. $[\ldots 4 n]$ & $\begin{array}{ll}\text { g. } & {[\ldots 4 n] \text { so they }\{\text { DURp4) called }} \\
& \{\text { DUAt } 11\} \text { repeatedly } \\
& \{\text { DUAt } 13\}\end{array}$ & g. $[\ldots 4 \mathrm{n}]$ \\
\hline 4 & $\mathrm{~h}$. & $\begin{array}{l}\text { h. until he }\{\text { IKRp3 }\} \text { stopped } \\
\{\text { IKAb9 }\}\end{array}$ & $\mathrm{h}$. \\
\hline
\end{tabular}




\begin{tabular}{|c|c|c|c|}
\hline $\begin{array}{l}\text { Line } \\
\text { No. }\end{array}$ & $\begin{array}{c}\text { Iktomi and the Ducks } \\
\text { ZITKALA-SA }\end{array}$ & $\begin{array}{c}\text { Ikto'mi Tricks the Pheasants } \\
\text { DELORIA }\end{array}$ & $\begin{array}{c}\text { Iktomi } \\
\text { WALKER }\end{array}$ \\
\hline 4 & i. & $\begin{array}{l}\text { i. and then they }\{\text { DURp4 }\} \text { asked, } \\
\{\text { DUAb } 8\}\end{array}$ & i. and asked $\{$ DUAb8 $\}$, \\
\hline 4 & j. Old fellow $\{$ IKRp5 $\}$, & j. & j. $\quad$ Older brother $\{$ IKRp5 $\}$, \\
\hline 4 & $\begin{array}{l}\text { k. Pray tell us }\{\text { DURp4 }\} \text { what } \\
\text { you }\{\text { IKRp4\} carry in your } \\
\{\text { IKRp4\} blanket. }\{\text { IKRp } 1\}\end{array}$ & $\begin{array}{l}\mathrm{k} . \text { "What's that you }\{\text { IKRp4\} carry } \\
\text { on your }\{\text { IKRp4\} back?" } \\
\{\text { DUAt } 12\}\end{array}$ & $\begin{array}{ll}\mathrm{k} . & \text { where are you }\{\text { IKRp } 4\} \\
& \text { going?" }\{\text { DUAt } 12\}\{\text { DUAt } 13\}\end{array}$ \\
\hline 4 & $\begin{array}{l}\text { 1. Do not hurry off! Stop! halt!" } \\
\text { \{DUAt } 13\} \text { urged one \{SKRp4\} } \\
\text { of the singers \{DURp2\}. }\end{array}$ & 1. & 1 . \\
\hline 4 & $\begin{array}{l}\mathrm{m} . \\
{[\ldots 4 \mathrm{~g}] \text { " Stop ! stay! Show us }} \\
\\
\{\text { DURp4\} what is in your } \\
\{\text { IKRp1\} blanket!" \{DUAt13\} } \\
\text { cried out other voices } \\
\text { \{DUAb8\} \{DUAt12\} } \\
\{\text { DUAt } 11\} .\end{array}$ & m. $[\ldots 4 \mathrm{~g}]$ & $\mathrm{m} \cdot[\ldots 4 \mathrm{~g}]$ \\
\hline 4 & $\mathrm{n}$. & n. [It is said] & $\mathrm{n}$. \\
\hline 5 & 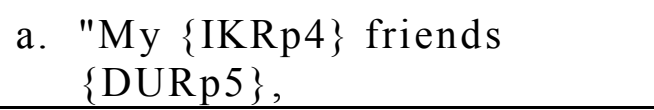 & a. & a. \\
\hline 5 & $\begin{array}{ll}\text { b. } & \text { I }\{\text { IKRp } 4\} \text { must not spoil } \\
& \text { your }\{\text { DURp } 4\} \text { dance. } \\
& \{\text { IKAb8 }\{\text { IKAt } 14\}\end{array}$ & b. & b. \\
\hline 5 & $\begin{array}{l}\text { c. Oh, you }\{\text { DURp4 }\} \text { would not } \\
\text { care to see if you }\{\text { DURp4\} } \\
\text { only knew what is in my } \\
\{\text { IKRp4\} blanket. \{IKAt } 11\} \\
\text { Sing on! dance on! }\end{array}$ & c. & c. \\
\hline
\end{tabular}




\begin{tabular}{|c|c|c|c|}
\hline $\begin{array}{l}\text { Line } \\
\text { No. }\end{array}$ & $\begin{array}{c}\text { Iktomi and the Ducks } \\
\text { ZITKALA-SA }\end{array}$ & $\begin{array}{c}\text { Ikto'mi Tricks the Pheasants } \\
\text { DELORIA }\end{array}$ & $\begin{array}{c}\text { Iktomi } \\
\text { W ALKER }\end{array}$ \\
\hline 5 & $\begin{array}{ll}\text { d. } & \text { I }\{\text { IKRp4 }\} \text { must not show } \\
& \text { you }\{\text { DURp4\} what I } \\
& \{\text { IKRp4\} carry on my } \\
& \{\text { IKRp4\} back," \{IKAt15\} } \\
& \text { answered IKTOMI }\{\text { IKRp1\}, } \\
& \text { nudging his }\{\text { IKRp3 } \text { own } \\
& \text { sides with his }\{\text { IKRp3 } \\
& \text { elbows }\{\text { IKAt } 15\} .\end{array}$ & d. & d. \\
\hline 5 & $\begin{array}{l}\text { e. This reply broke up the ring } \\
\{\text { DURp } 1\} \text { entirely. }\end{array}$ & e. & e. \\
\hline 5 & $\begin{array}{l}\text { f. Now all the ducks }\{\text { DURp } 1\} \\
\text { crowded }\{\text { DUAt } 11\} \text { about } \\
\text { IKTOMI }\{\text { IKRp } 1\} .\end{array}$ & f. & f. \\
\hline 5 & $\begin{array}{l}\text { g. "We }\{\text { DURp4\} must see } \\
\text { what you }\{\text { IKRp4\} carry! } \\
\text { We }\{\text { DURp4\} must know what } \\
\text { is in your }\{\text { IKRp4\} blanket!" } \\
\{\text { DUAt13\} they }\{\text { DURp4\} } \\
\text { shouted }\{\text { DUAb8\} } \\
\{\text { DUAt12\} }\{\text { DUAt11\} in } \\
\text { both his }\{\text { IKRp3\} ears. } \\
\text { Some }\{\text { DURp4\} even } \\
\text { brushed their }\{\text { DURp4 } \text { wings } \\
\text { \{DURp1\} against the mysteri- } \\
\text { ous bundle. }\end{array}$ & g. & g. \\
\hline 5 & h. $[\ldots 5 \mathrm{j}]$ & h. $[\ldots 5 \mathrm{j}]$ & $\begin{array}{c}\text { h. }[\ldots 5 \mathrm{j}] \text { "I'm }\{\text { IKRp4\} } 4 \text { going } \\
\text { over there }\{\text { IKAb } 8\}\end{array}$ \\
\hline
\end{tabular}




\begin{tabular}{|c|c|c|c|}
\hline $\begin{array}{l}\text { Line } \\
\text { No. }\end{array}$ & $\begin{array}{c}\text { Iktomi and the Ducks } \\
\text { ZITKALA-SA }\end{array}$ & $\begin{array}{c}\text { Ikto'mi Tricks the Pheasants } \\
\text { DELORIA }\end{array}$ & $\begin{array}{c}\text { Iktomi } \\
\text { WALKER }\end{array}$ \\
\hline 5 & 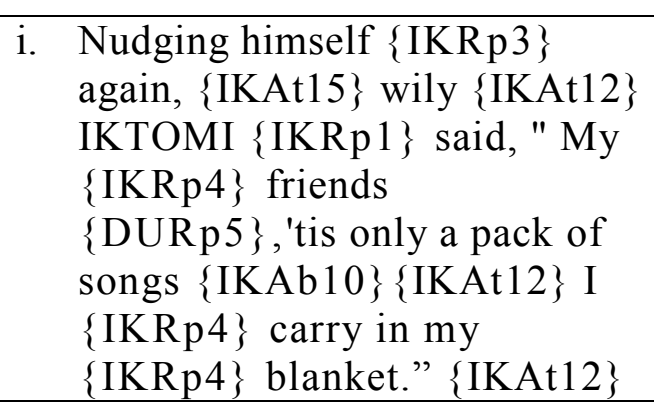 & $\begin{array}{l}\text { i. - } \text { - These ? Why they are just } \\
\text { some silly little songs. } \\
\{\text { IKAb8 }\{\text { IKAb10\} }\{\text { IKAt } 12\}\end{array}$ & $\begin{array}{l}\text { i. with these songs }\{\text { IKAb10 } \\
\quad\{\text { IKAt } 12\}\end{array}$ \\
\hline 5 & j. $[\ldots 5 \mathrm{~h}]$ & $\begin{array}{l}\mathrm{j} . \text { There is to be a dance } \\
\quad\{\text { IKAb } 10\}[\ldots 5 \mathrm{~h}] \text { farther up, } \\
\\
\{\text { IKTOP }\}\end{array}$ & j. $[\ldots 5 \mathrm{~h}]$ \\
\hline 5 & $\mathrm{k}$. & $\begin{array}{l}\text { k. and I've }\{\text { IKRp4 }\} \text { been sent for. } \\
\text { That's why I }\{\text { IKRp4 }\} \text { am in a } \\
\text { rush; }\end{array}$ & $\begin{array}{l}\text { k. and I'm }\{\text { IKRp4 }\} \text { in a hurry," } \\
\text { he }\{\text { IKRp } 3\} \text { said. }\end{array}$ \\
\hline 5 & 1. & $\begin{array}{c}\text { 1. now what are they }\{\text { DURp4\} } \\
\text { after?" he }\{\text { IKRp3 }\} \text { added. }\end{array}$ & 1. \\
\hline 5 & $\mathrm{~m}$. & m. [It is said] & $\mathrm{m}$. \\
\hline 6 & a. & a. "O, elder brother $\{\operatorname{IKRp5}\}$, & $\begin{array}{l}\text { a. "Older brother }\{\text { IKRp } 5\}, \\
\text { you're }\{\text { IKRp } 4\} \text { in a hurry, } \\
\{\text { DUAt } 14\}\end{array}$ \\
\hline 6 & $\begin{array}{l}\text { b. } \text { "Oh, then let us }\{\text { DURp4 }\} \text { hear } \\
\text { your }\{\text { IKRp } 4\} \text { songs!" } \\
\text { \{DUAt13\} cried the curious } \\
\{\text { DUAt } 12\} \text { ducks }\{\text { DURp } 1\} .\end{array}$ & $\begin{array}{l}\text { b. come over here and sing us } \\
\text { \{DURp4\} one tiny little song; } \\
\text { just one," }\{\text { DUAt } 13\} \text { they } \\
\{\text { DURp } 4\} \text { said }\end{array}$ & $\begin{array}{l}\text { b. but sing one for us }\{\text { DURp } 4\} \\
\text { and then go," }\{\text { DUAt } 13\} \text { they } \\
\{\text { DURp } 4\} \text { said. }\end{array}$ \\
\hline 6 & c. & $\begin{array}{l}\text { c. and again he }\{\text { IKRp3 }\} \text { said, } \\
\text { "Indeed not! }\{\text { IKAt13\} I've }\end{array}$ & c. \\
\hline
\end{tabular}




\begin{tabular}{|c|c|c|c|}
\hline $\begin{array}{l}\text { Line } \\
\text { No. }\end{array}$ & $\begin{array}{l}\text { Iktomi and the Ducks } \\
\text { ZITKALA-SA }\end{array}$ & $\begin{array}{c}\text { Ikto'mi Tricks the Pheasants } \\
\text { DELORIA }\end{array}$ & $\begin{array}{c}\text { Iktomi } \\
\text { W ALKER }\end{array}$ \\
\hline & & $\begin{array}{l}\{\text { IKRp4 }\} \text { just told you } \\
\{\text { DURp4 }\} \text { I'm }\{\text { IKRp4 }\} \text { in a } \\
\text { hurry. What's the idea?" and he } \\
\{\text { IKRp3 }\} \text { walked on. }\{\text { IKAb9\} }\end{array}$ & \\
\hline 6 & & d. [It is said] & \\
\hline
\end{tabular}




\section{Appendix C}

\section{Character Traits Coding Template}

\section{Category Code Subcategory: Defining traits}

\begin{tabular}{|c|c|c|}
\hline \multirow[t]{7}{*}{$\begin{array}{c}\text { Representation } \\
(R p)\end{array}$} & & \\
\hline & Rp1 & $\begin{array}{l}\text { Nonhuman: references to animal, plant, mineral, or } \\
\text { elemental }\end{array}$ \\
\hline & Rp2 & Human: references indicating inherently human traits \\
\hline & Rp3 & Pronoun: Male reference ( $3^{\text {rd }}$ person singular masculine) \\
\hline & Rp4 & $\begin{array}{l}\text { Pronoun: Other (includes pronouns other than } 3^{\text {rd }} \text { person } \\
\text { singular masculine) }\end{array}$ \\
\hline & Rp5 & $\begin{array}{l}\text { Recognition: Addressed by name (usu. relative term): } \\
\text { indicates recognition as an individual }\end{array}$ \\
\hline & Rp6 & $\begin{array}{l}\text { Powerlessness: indicates ineffectiveness to direct one's } \\
\text { own fate }\end{array}$ \\
\hline
\end{tabular}

Abilities

(Ab)

Ab7 Skills: ability to manipulate tools or objects

Ab8 Sensory/rational/emotive: modes of sense, perception, and expression

Ab9 Locomotion: mobility and conscious movement

Ab10 Willful intent: ability to commit acts with conscious rationality

\section{Attributes}

(At)

At11 Appearance/personality/inclination: personal presentation to others

At12 Disposition: mental and emotionally driven impulses

At13 Behavior/Attitude toward others-Negative: indicate separateness and undesirability among others

At14 Behavior/Attitude toward others-Positive: indicate inclusiveness and identification with others

At15 Egocentrism: self-centered behavior and attitude 


\section{Appendix D}

\section{Polarity-Code/Character-Code Correspondence Template}

\begin{tabular}{|c|c|c|c|c|c|c|c|c|c|c|c|}
\hline \multirow{3}{*}{ 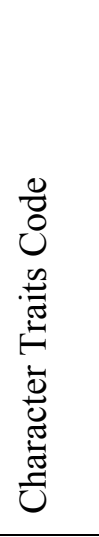 } & & \multicolumn{10}{|c|}{ Polarity-Code Pairs } \\
\hline & & 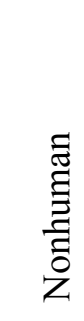 & 壱 & 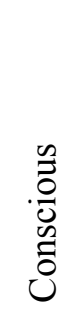 & 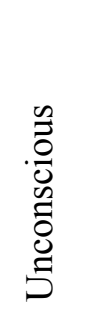 & 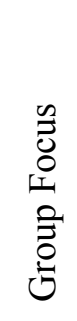 & 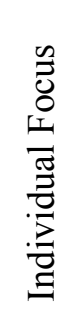 & 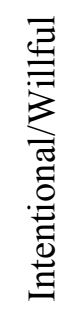 & 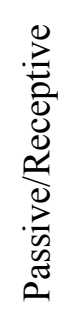 & $\begin{array}{l}\text { ठ্ } \\
\stackrel{\Xi}{\Xi} \\
Z\end{array}$ & 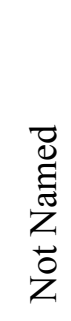 \\
\hline & $\begin{array}{c}\text { Character Code } \\
\text { Descriptor }\end{array}$ & $\mathrm{NH}$ & $\mathrm{HU}$ & $\mathrm{CO}$ & $\mathrm{UN}$ & GP & IN & IT & PA & NM & NN \\
\hline & Representation & & & & & & & & & & \\
\hline Rp1 & Nonhuman & $\mathrm{x}$ & & & & & & & & $\mathrm{x}$ & $\mathrm{x}$ \\
\hline Rp2 & Human & & $\mathrm{x}$ & & & & & & & $\mathrm{x}$ & \\
\hline Rp3 & Pronoun-male reference & $\mathrm{x}$ & $\mathrm{x}$ & & & & $\mathrm{x}$ & & & & \\
\hline Rp4 & Pronoun-other & $\mathrm{x}$ & $\mathrm{x}$ & & & $\mathrm{x}$ & $\mathrm{x}$ & & & & \\
\hline Rp5 & Recognition & $\mathrm{x}$ & $\mathrm{x}$ & $\mathrm{x}$ & & $\mathrm{x}$ & $\mathrm{x}$ & & & $\mathrm{x}$ & \\
\hline \multirow[t]{2}{*}{ Rp6 } & Powerlessness & & $\mathrm{x}$ & $\mathrm{x}$ & & & $\mathrm{x}$ & & $\mathrm{x}$ & & \\
\hline & Abilities & & & & & & & & & & \\
\hline $\mathrm{Ab} 7$ & Skills & & $\mathrm{x}$ & $\mathrm{x}$ & & & $\mathrm{x}$ & $\mathrm{x}$ & & & \\
\hline Ab8 & $\begin{array}{l}\text { Sensory/rational/ } \\
\text { emotive }\end{array}$ & $\mathrm{x}$ & $\mathrm{x}$ & $\mathrm{x}$ & & & $\mathrm{x}$ & $\mathrm{x}$ & $\mathrm{x}$ & & \\
\hline Ab9 & Locomotion & $\mathrm{x}$ & $\mathrm{x}$ & & & & & $\mathrm{x}$ & & & \\
\hline \multirow[t]{2}{*}{$\mathrm{Ab} 10$} & Willful intent & & $\mathrm{x}$ & $\mathrm{x}$ & & & $\mathrm{x}$ & $\mathrm{x}$ & & & \\
\hline & Attributes & & & & & & & & & & \\
\hline At11 & $\begin{array}{l}\text { Appearance/personality/ } \\
\text { inclination }\end{array}$ & $\mathrm{x}$ & $\mathrm{x}$ & & $\mathrm{x}$ & & & & & & \\
\hline At12 & Disposition & & $\mathrm{x}$ & $\mathrm{x}$ & & & & $\mathrm{x}$ & & & \\
\hline At13 & $\begin{array}{l}\text { Behavior/attitude- } \\
\text { negative }\end{array}$ & & $\mathrm{x}$ & $\mathrm{x}$ & & & $\mathrm{x}$ & $\mathrm{x}$ & & & \\
\hline At14 & $\begin{array}{l}\text { Behavior/attitude- } \\
\text { positive }\end{array}$ & & $\mathrm{x}$ & $\mathrm{x}$ & & & & $\mathrm{x}$ & & & \\
\hline At15 & Egocentrism & & $\mathrm{x}$ & $\mathrm{x}$ & & & $\mathrm{x}$ & $\mathrm{x}$ & & & \\
\hline $\begin{array}{l}\text { Total } \\
\text { Types }\end{array}$ & $\begin{array}{l}\text { ossible Code } \\
\text { or Groups: }\end{array}$ & 7 & 14 & 9 & 1 & 2 & 9 & 8 & 2 & 3 & 1 \\
\hline
\end{tabular}

